ANDRÉ MARCORIN DE OLIVEIRA

Estimating and control of Markov jump linear systems with partial observation of the operation mode 


\section{Estimating and control of Markov jump linear systems with partial observation of the operation mode}

Thesis presented for the degree of Doctor of Science to the Escola Politécnica of Universidade de São Paulo.

Concentration field:

Systems Engineering

Advisor:

Prof. Dr. Oswaldo Luiz do Valle Costa 
Autorizo a reprodução e divulgação total ou parcial deste trabalho, por qualquer meio convencional ou eletrônico, para fins de estudo e pesquisa, desde que citada a fonte.

Este exemplar foi revisado e corrigido em relação à versão original, sob responsabilidade única do autor e com a anuência de seu orientador.

São Paulo, de de

Assinatura do autor:

Assinatura do orientador:

\section{Catalogação-na-publicação}

Oliveira, André Marcorin

Estimating and control of Markov jump linear systems with partial observation of the operation mode / A. M. Oliveira -- versão corr. -- São Paulo, 2019.

$175 \mathrm{p}$.

Tese (Doutorado) - Escola Politécnica da Universidade de São Paulo. Departamento de Engenharia de Telecomunicações e Controle.

1.Sistemas lineares 2.Cadeias de Markov 3.Controle ótimo 4.Controle robusto 5.Otimização convexa I.Universidade de São Paulo. Escola Politécnica. Departamento de Engenharia de Telecomunicações e Controle II.t. 
To Gabi, João, and Bibi 


\section{ACKNOWLEDGEMENT}

First, I would like to thank my family. To Gabriela, for always being there when I needed most, and to João and Bibi for showing me how kindness and innocence are features that should not be lost in one's heart. I love you all. To my mother, my role model, the person that I always look up to thanks to her strength, determination, and courage. There are no words in any language that I could use to describe how grateful I am to her. To my brother, Leonardo, and to my sisters Flavia and Cassia. Even though we are now (too) far away, you will always be near. To my father for teaching me values of courage, honesty, and how to see life more lightly (I must admit that I am a slow learner at that). To my grandfathers and grandmothers that believed in me in this career that I chose, and to my friends that supported me throughout these years.

I would like to thank my advisor, Oswaldo. We had a great and very productive time this last couple of years. I learned a lot, not only in the technical aspects (that I surely need to learn more), but also in how to be a better researcher. This is not an easy task, and I am still learning. Also, in backing the idea and great opportunity of going to France and helping me in every technical aspect and difficulty I had these last three years.

Thanks also to my advisor in France, Jamal, and to Romain, Constantin, and Vineeth. They allowed me this unique opportunity to join their research group at CRAN in this last year. I learned A LOT in terms of research and colleagueship, professionally and personally and will take all these lessons with me from now on. Merci beaucoup à vous et aussi à tous mes collègues au CRAN!

My staying in France would be a completely different story if it wasn't for my friends, Bruno and Siffedine. We shared a lot of fun and laughs, and they were always there when I needed them. I will miss this time we spent together. Thanks also to Arthur, Elaine, Giuseppe, and Mathieu for the laughs. A special thanks also to Jomphop, Nico, Tommaso, Prisca, Hervé, Harry, Daniele, Christine, and Marc at CRAN, as well as to my colleagues and friends at USP, Leonardo, Yuri, Fabio, Andres, Lina, Gabriel, Fernando, and Prof. Bruno!

Finally, I would like to express my gratitude to the members of my examination committee and to thank the São Paulo Research Foundation (FAPESP) for the financial support provided to this project through the grant 2015/09912-8, including my staying at the University of Lorraine/CRAN through the grant 2017/06358-5, and for believing and supporting the research in the state of São Paulo. 


\section{RESUMO}

Nesta tese, apresentamos algumas contribuições para a teoria de sistemas lineares com saltos markovianos em um contexto de observação parcial da cadeia de Markov. Consideramos que o estado da cadeia de Markov não pode ser medido, porém existe uma variável observada que pode modelar um fenômeno assíncrono entre a aplicação e a planta, ou ainda um dispositivo de detecção de falhas simples. Através desse modelo, investigamos o problema da síntese de controladores e filtros que dependem somente da variável observada no contexto das teorias de controle $\mathcal{H}_{2}, \mathcal{H}_{\infty}$, e misto $\mathcal{H}_{2} / \mathcal{H}_{\infty}$. Exemplos numéricos e aplicações acadêmicas são apresentadas no âmbito dos sistemas de controle tolerantes a falhas e dos sistemas de controle através da rede.

Palavras-chave: Sistemas lineares. Cadeias de Markov. Controle ótimo. Controle Robusto. Otimização convexa. 


\begin{abstract}
In this thesis, we present some contributions to the Markov jump linear systems theory in a context of partial information on the Markov chain. We consider that the state of the Markov chain cannot be measured, but instead there is only an observed variable that could model an asynchronous phenomenon between the application and the plant, or a simple fault detection and isolation device. In this formulation, we investigate the problem of designing controllers and filters depending only on the observed variable in the context of $\mathcal{H}_{2}, \mathcal{H}_{\infty}$, and mixed $\mathcal{H}_{2} / \mathcal{H}_{\infty}$ control theory. Numerical examples and academic applications are presented for active-fault tolerant control systems and networked control systems.
\end{abstract}

Keywords: Linear systems. Markov chains. Optimal control. Robust control. Convex optimization. 


\section{LIST OF FIGURES}

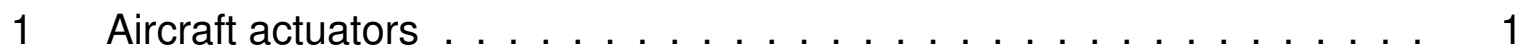

2 The Markov chain for the unmanned aircraft modes of operation $\ldots .2$

3 Schematic diagram for AFTCS $\ldots \ldots \ldots \ldots \ldots$

4 General control system structure $\ldots \ldots \ldots \ldots \ldots$

5 A possible trajectory of $\theta(k)$ (Markov chain, in black) and $\hat{\theta}(k)$ (measured variable, in grey $\ldots \ldots \ldots \ldots \ldots . \ldots \ldots$

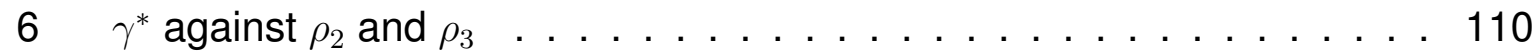

$7 \quad \delta^{*}$ and $\left\|\mathcal{G}_{K}\right\|_{\infty}$ against $\rho \ldots \ldots \ldots \ldots \ldots \ldots \ldots \ldots \ldots \ldots$

$8 \gamma^{*}$ against $\delta$ for $\rho=1$ (black line), $\rho=0.7$ (darker grey line), and $\rho=0.5$ (brighter grey line) . . . . . . . . . . . . . . . . . . . 112

$9 \gamma^{*}$ (black line) and $\left\|\mathcal{G}_{K}\right\|_{2}$ (dashed black line) against $\rho \ldots \ldots \ldots 112$

$10 \gamma^{*}$ for $\delta=23.5$ against $\epsilon$ for the vertices of $\Upsilon$ given in (8.3) $\ldots \ldots \ldots 113$

$11 \gamma^{*}$ (black line) and $\left\|\mathcal{G}_{c}\right\|_{2}$ (dashed black line) against $\rho \ldots \ldots \ldots$

$12 \delta^{*}$ (black line) and $\left\|\mathcal{G}_{c}\right\|_{\infty}$ (dashed black line) against $\rho \ldots \ldots \ldots$

$13 \mathbf{E}\left(\left\|e_{2}(k)\right\|^{2}\right) \pm \sigma$ in function of $k \ldots \ldots \ldots \ldots \ldots$

$14 \mathbf{E}\left(x_{1}(k)\right)$ (black line) and $\mathbf{E}\left(z_{f}(k)\right)$ (grey line) against $k$ for the $\mathcal{H}_{\infty}$ filter . 121

15 Schematic of the WNCS . . . . . . . . . . . . . . . . . . . . . . 122

16 The SATI working scheme . . . . . . . . . . . . . . . . . . . 123

17 The Markov chain for $\tau(k) \ldots \ldots \ldots \ldots \ldots$

18 Solution (circles) or unfeasible (crosses) pairs $(N, \eta)$ of the conditions of Theorem 8.2, for $\delta=0.2 \ldots \ldots \ldots \ldots$. . . . . . . . . 130

19 Minimum $\eta$ feasible for the SATI controller (black line) and the LQR controller (dashed grey line). . . . . . . . . . . . . . . . . . . 132 


\section{LIST OF ABBREVIATIONS}

(A)FTCS (Active) fault-tolerant control systems

BMI Bilinear matrix inequalities

CARE Coupled algebraic Riccati equation

FDI Fault-detection and isolation

HMC Hidden Markov chain

HMM Hidden Markov model

JLQ Jump linear quadratic

LMI Linear matrix inequalities

LMMSE Linear minimum mean square error

LQG Linear quadratic Gaussian

$\mathrm{LQ}(\mathrm{R}) \quad$ Linear quadratic (regulator)

LTI Linear time-invariant

MJLS Markov jump linear systems

MM $\quad$ Multiple models

SATI Stochastic allowable transmission interval

SS Stochastic stability

MATI Maximum allowable transmission interval

(W)NCS (Wireless) networked control systems 


\section{LIST OF SYMBOLS}

$\Omega$

$\mathfrak{F}, \mathfrak{F}_{k}, \hat{\mathfrak{F}}_{k}$

Prob

$\theta, \theta(k)$

$p_{i j}$

$\mathbb{P}$

$\mu_{i}, \nu_{i}$

$\hat{\theta}, \hat{\theta}(k)$

$\alpha_{i l}$

$\Upsilon$

$\mathbf{E}(\cdot)$

$\mathbf{E}(\cdot \mid \cdot)$

$\sigma$

$\mathcal{N}\left(a, \sigma^{2}\right)$

$\mathcal{U}(a, b)$

$1_{A}$

$\mathbb{Y}, \mathbb{X}$

$\mathbb{B}(\mathbb{Y}, \mathbb{X}), \mathbb{B}(\mathbb{Y})$

$\mathbb{R}^{n}$

$\mathbb{B}\left(\mathbb{R}^{n}, \mathbb{R}^{m}\right), \mathbb{B}\left(\mathbb{R}^{n}\right)$

$\langle\cdot ; \cdot\rangle$

$\|w(k)\|$

$\|w\|_{2}$
Sample space.

$\sigma$-fields.

Probability measure.

Markov chain.

Transition probability from mode $i$ to $j$.

Transition probability matrix.

Initial and limiting probabilities of mode $i$.

Measured variable.

Conditional probability of obtaining $\hat{\theta}(k)=l$ given that $\theta(k)=i$.

Conditional probability matrix.

Expected value / mean value operator.

Conditional expected value operator.

Standard deviation.

Normal distribution with mean $a$ and variance $\sigma^{2}$.

Uniform distribution in the interval $[a, b]$.

Indicator function of the event $A$.

Banach spaces.

Banach space of $\mathbb{Y}$ into $\mathbb{X}$.

Euclidean space of all $n$-dimensional vectors.

Banach space of $\mathbb{R}^{n}$ into $\mathbb{R}^{m}$.

Inner product in a Hilbert Space.

Euclidean norm of the vector $w(k)$ at time $k$.

$l_{2}$-norm of the sequence $w$. 


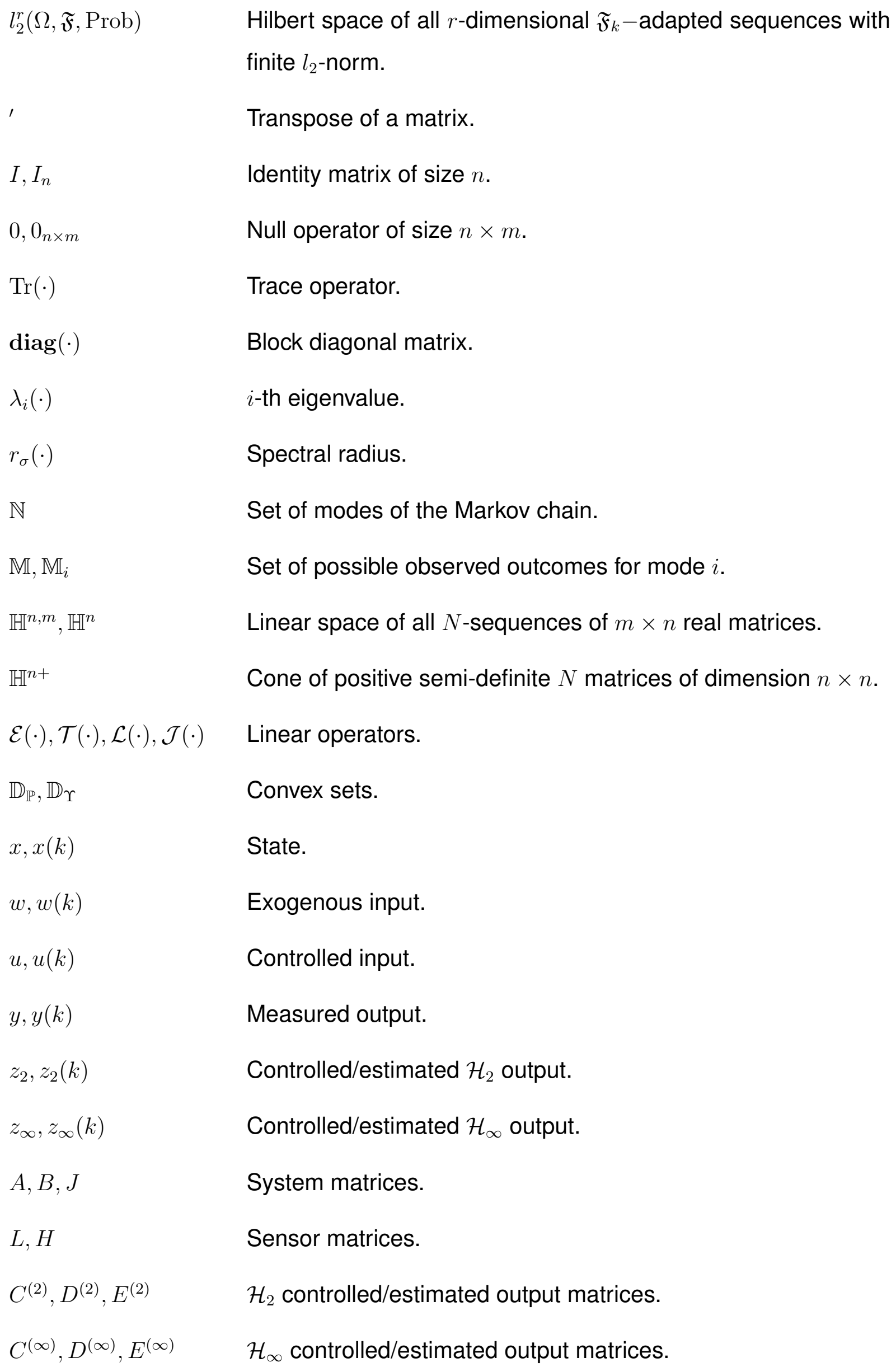



$\hat{\mathbf{J}}, \mathbf{J}$
Controllability Gramian.
$\hat{\mathrm{C}}, \mathrm{C}$
Observability Gramian.
$\|\mathcal{G}\|_{2}$
$\mathcal{H}_{2}$ norm of system $\mathcal{G}$.
(Best) guaranteed cost, upper bound of the $\mathcal{H}_{2}$ norm.
$\|\mathcal{G}\|_{\infty}$
$\mathcal{H}_{\infty}$ norm of system $\mathcal{G}$.
$\delta^{*}, \delta$
(Best) guaranteed cost, upper bound of the $\mathcal{H}_{\infty}$ norm.
$K,\left\|\mathcal{G}_{K}\right\|_{a}$
State-feedback controller, closed-loop system norm controlled by $K$, for $a \in\{2, \infty\}$.
Set of stochastically stabilizing state-feedback controllers.
$\mathcal{F},\left\|\mathcal{G}_{f}\right\|_{a}$
Filter, norm of the extended system composed by the filter and the plant, for $a \in\{2, \infty\}$.
F
$\mathbb{F}$
$\mathcal{C},\left\|\mathcal{G}_{c}\right\|_{a}$
$\mathbb{C}$
$\xi, \xi_{2}, \xi_{\infty}$
$\Xi, \Xi_{2}, \Xi_{\infty}$
Static output feedback controller.
Set of admissible filters/static output feedback controllers.
Dynamic output feedback controller, closed-loop system norm controlled by $\mathcal{C}$, for $a \in\{2, \infty\}$.
Set of admissible dynamic output feedback controllers.
Set of variables.
Set of solutions of a given inequality set. 


\section{CONTENTS}

1 Introduction 1

2 Literature review $\quad 6$

2.1 The complete observation case . . . . . . . . . . . . . 7

2.2 Mode-dependent filtering and control . . . . . . . . . . . . . . . . . 9

2.2 .1 Filtering . . . . . . . . . . . . . . . . . . . . . . . . . . . 9

2.2.2 Dynamic and static output feedback control . . . . . . . . . . . . 10

2.3 The case of partial observation on $\theta \ldots \ldots \ldots 11 \ldots \ldots$

2.3.1 Alternative observation structures on $\theta \ldots \ldots 12$

2.4 The case of partial observation on both $x$ and $\theta \ldots \ldots \ldots 14$

2.4.1 Filtering . . . . . . . . . . . . . . . . . . . . . 14

2.4.2 Dynamic and static output feedback control . . . . . . . . . . . . 15

2.5 Contributions of this thesis . . . . . . . . . . . . . . . . . 16

3 Preliminaries $r$

3.1 Notation . . . . . . . . . . . . . . . . . . . . . . . . . . . . . . . 19

3.2 The hidden MJLS theory . . . . . . . . . . . . . . . . . . . . 20

3.2.1 Hidden Markov Jump Linear Systems . . . . . . . . . . . . . . . 20

3.2.2 Stability for hidden MJLS . . . . . . . . . . . . . . . . . 24

3.2.3 The $\mathcal{H}_{2}$ norm for hidden MJLS $\ldots \ldots \ldots$

3.2.4 The $\mathcal{H}_{\infty}$ norm for hidden MJLS . . . . . . . . . . . . . . . . . 33

3.3 Final remarks . . . . . . . . . . . . . . . . . . . 36

4 State-feedback control for hidden MJLS 37 
4.1 Problem formulation $\ldots \ldots \ldots \ldots \ldots \ldots \ldots \ldots$

4.2 Stabilizing state-feedback control for hidden MJLS . . . . . . . . 40

4.3 The $\mathcal{H}_{2}$ state-feedback control for hidden MJLS . . . . . . . . . . 42

4.4 The $\mathcal{H}_{\infty}$ state-feedback control for hidden MJLS $\ldots \ldots \ldots \ldots \ldots$

4.5 The mixed $\mathcal{H}_{2} / \mathcal{H}_{\infty}$ state-feedback control for hidden MJLS $\ldots \ldots$. . 47

4.6 State-feedback control for the cluster case and robust control . . . . . 49

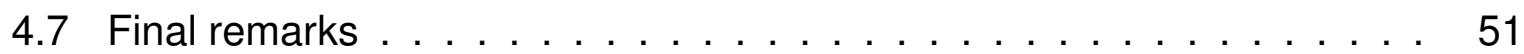

5 Filtering for hidden MJLS 52

5.1 Problem formulation $\ldots \ldots \ldots \ldots \ldots \ldots \ldots \ldots \ldots \ldots$

$5.2 \mathcal{H}_{2}$ filtering for hidden MJLS $\ldots \ldots \ldots \ldots \ldots \ldots \ldots$

$5.3 \mathcal{H}_{\infty}$ filtering for hidden MJLS $\ldots \ldots \ldots \ldots \ldots \ldots$

5.4 The mixed $\mathcal{H}_{2} / \mathcal{H}_{\infty}$ filtering for hidden MJLS $\ldots \ldots \ldots \ldots$

5.5 Filtering for the cluster case and robust filtering $\ldots \ldots \ldots$. . . . 64

5.6 Filtering for the Bernoulli case for hidden MJLS . . . . . . . . . 66

5.7 Final remarks $\ldots \ldots \ldots \ldots \ldots \ldots \ldots \ldots \ldots \ldots$

6 Static output feedback control for hidden MJLS 72

6.1 Problem formulation $\ldots \ldots \ldots \ldots \ldots \ldots \ldots \ldots$

$6.2 \mathcal{H}_{2}$ static output feedback control for hidden MJLS $\ldots \ldots \ldots \ldots .74$

$6.3 \mathcal{H}_{\infty}$ static output feedback control for hidden MJLS $\ldots \ldots \ldots \ldots$

6.4 The mixed $\mathcal{H}_{2} / \mathcal{H}_{\infty}$ static output feedback control . . . . . . . . . 79

6.5 Static output feedback control for the cluster case and robust control . . 81

6.6 Final remarks $\ldots \ldots \ldots \ldots \ldots \ldots \ldots \ldots \ldots \ldots$ 
7.1 Problem formulation $\ldots \ldots \ldots \ldots \ldots \ldots \ldots$

$7.2 \mathcal{H}_{2}$ dynamic output feedback control for hidden MJLS . . . . . . . 88

7.3 $\mathcal{H}_{\infty}$ dynamic output feedback control for hidden MJLS $\ldots \ldots \ldots .97$

7.4 The mixed $\mathcal{H}_{2} / \mathcal{H}_{\infty}$ dynamic output feedback control $\ldots \ldots \ldots \ldots$

7.5 Dynamic output feedback control for the cluster case and robust control 104

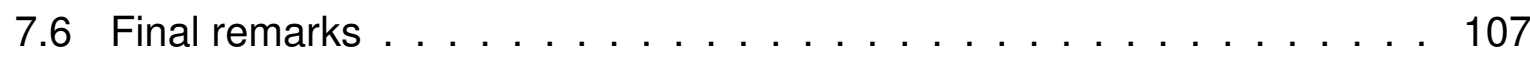

$\begin{array}{lll}8 & \text { Academic applications } & 108\end{array}$

8.1 Unmanned Aircraft . . . . . . . . . . . . . . . . . . . . . 108

8.1.1 State-feedback control . . . . . . . . . . . . . . . . . 109

8.1.2 Dynamic output feedback control . . . . . . . . . . . . . . . 114

8.2 Mass-spring-damper system $\ldots \ldots \ldots \ldots \ldots \ldots$

8.3 Stochastic Allowable Transmission Intervals - The $(\eta, \delta)$-SATI $\ldots \ldots .121$

9 Conclusion and perspectives 133

$\begin{array}{ll}\text { References } & 136\end{array}$

$\begin{array}{ll}\text { Appendix A - Auxiliary results } & 147\end{array}$

Appendix B - Stabilizability, Observability, and Coupled Algebraic Riccati Equations

Appendix C - Numerical results of Chapter 8 


\section{INTRODUCTION}

Control systems are now a constant and essential presence in the everyday life, from active suspension systems for cars to the very complicated, and critical, applications such as airplane and satellite operations, among others. The merit of these achievements that aim to ease our life and to expand the limits of our knowledge comes also with a significant burden that is felt whenever the human life and the environment are at stake. There are numerous cases in which the use of technology led to catastrophic results, for example, the Chernobyl disaster, that was analyzed through the lens of the control theory in the essay (STEIN, 2003), or the many flight incidents that were caused by human mistake, system failures, or the conjunction of both.

The study of systems subject to abrupt changes in their dynamics is crucial for modeling and detecting the anomalous behavior that may arise in the presence of faults, and consequently acting on the system to ensure a safe operation. In this context, the so-called Markov jump linear systems (MJLS) has attracted a great deal of attention in the literature in the past decades, in part due to some desirable characteristics presented such as random dynamic changes, among others. As a compelling example of an MJLS, consider an unmanned aircraft whose actuators are the aileron, the rudder, and the right and left elevators, as shown in Figure 1. In the case in which the

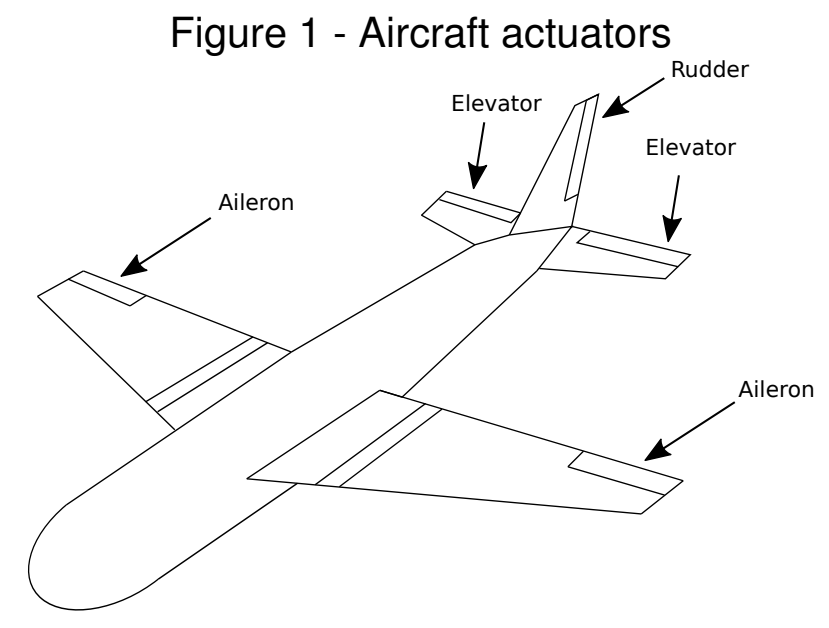

Source: Adapted from (DUCARD, 2009).

aileron and/or the rudder are subject to failures, and these changes can be modeled by a Markov chain, we can directly rely on the MJLS theory. We may consider that 
Figure 2 - The Markov chain for the unmanned aircraft modes of operation

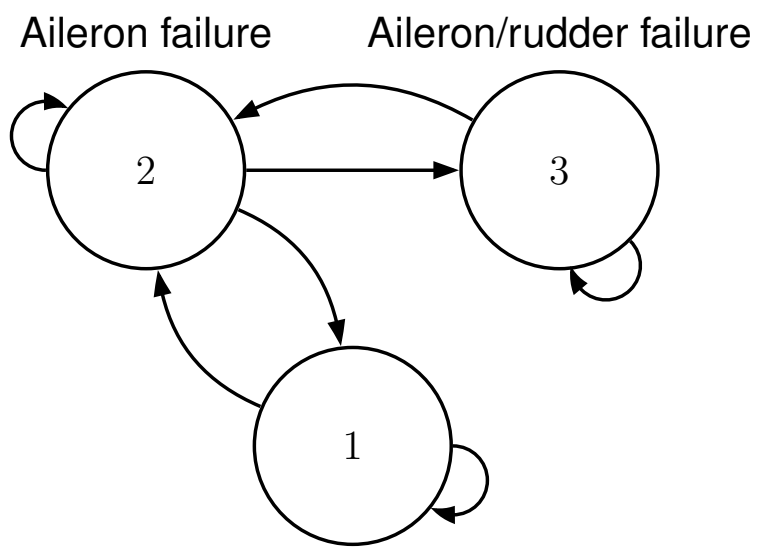

Nominal mode

Source: Author.

there is a nominal state representing the case without faults, another state in which the aileron command is not working, and a third situation in which the rudder command is inverted in addition to the aileron failure. A possible Markov chain for this model is shown in Figure 2, as described in (COSTA; FRAGOSO; TODOROV, 2015). For each mode of operation presented in the graph of Figure 2, we have a well-defined collection of subsystems that we could use for modeling and control our system.

Considering a more practical aspect, the effort to render systems more reliable has also increased in the past decades, leading to the development of many significant fields of research that can be inserted in the broader context of fault-tolerant control systems (FTCS), see, for instance, (PATTON, 1997; ZHANG; JIANG, 2008; HWANG et al., 2010; JIANG; YU, 2012). Of special interest is the natural extension of the FTCS called active fault-tolerant control systems (AFTCS) that usually consider a fault detection and isolation (FDI) device, along with a switching control law that follows the output of the FDI. Figure 3 presents a general schematic diagram of an AFTCS.

In this context, an important bridge between the AFTCS and MJLS theory was built by assuming that the dynamic behavior (nominal and faulty modes of operation) can be satisfactorily modeled by some given MJLS, as well as considering switched control laws depending on variables that model the behavior of FDI algorithms, as can be seen in the works (MARITON, 1989; SRICHANDER; WALKER, 1993; MAHMOUD; JIANG; ZHANG, 2003), and the references therein. However, the use of MJLS in this type of applications comes also with a burden. The bulk of works in the literature considers 
Figure 3 - Schematic diagram for AFTCS

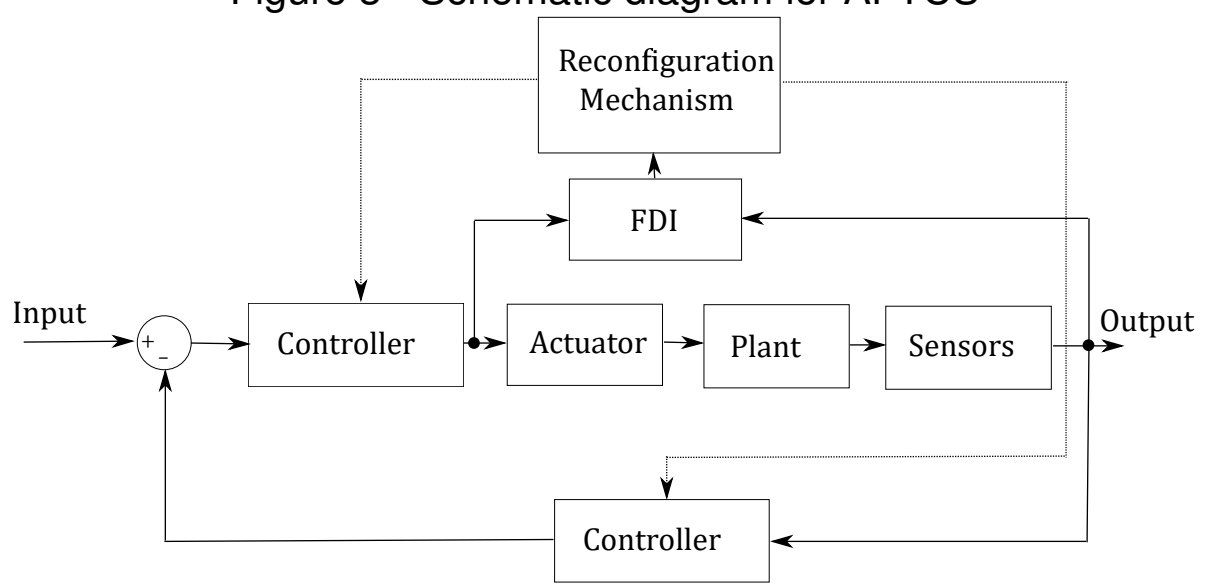

Source: Adapted from (MAHMOUD; JIANG; ZHANG, 2003).

that the Markov state can be perfectly measured, in part due to the great degree of complexity involving MJLS and problems with partial observation of the Markov chain. Yet, this is the same to assume that we would have access to a perfect FDI device, failure detector, or acknowledgment scheme (in the case of Networked Control Systems (NCS)), which is not a reasonable assumption for practical purposes.

Some workarounds do exist for tackling this issue, for instance, in NCS, the cluster case proposed in (VAL; GEROMEL; GONÇALVES, 2002) presents a reasonable method by assuming that an acknowledgment scheme can sense the packet drop-out. However, it is clear that no detection method is fail-safe, and can eventually present false-alarms or false negative flags. In this sense, there are simple models in the literature that could be used as alternatives, such as the ones presented in (SRICHANDER; WALKER, 1993; MAHMOUD; JIANG; ZHANG, 2003), the so-called "asynchronous behavior" of the work (WU et al., 2014), or the detector approach of (COSTA; FRAGOSO; TODOROV, 2015). In this sense, by properly considering the statistics of the detection algorithm, the AFTCS might be properly implemented, see, for instance, the discussion in (MAHMOUD; JIANG; ZHANG, 2003).

Bearing in mind, and motivated by, the discussion and study of AFTCS and MJLS, this work aims to contribute to the MJLS theory in a context of partial observation of the Markov chain. We focus on design in the detector approach formulation presented in (COSTA; FRAGOSO; TODOROV, 2015), that can also be viewed as an "asynchronous behavior", as put in (WU et al., 2017). Due to the resemblance of the method to the so-called hidden Markov models (HMM, specifically, hidden Markov chains, HMC), we 
refer generically to this model as hidden MJLS. The framework used in this work is that of the $\mathcal{H}_{2}$ and $\mathcal{H}_{\infty}$ control theory, and we try to formulate all the design problems in a general mixed $\mathcal{H}_{2} / \mathcal{H}_{\infty}$ control/filtering formulation, as shown in Figure 4.

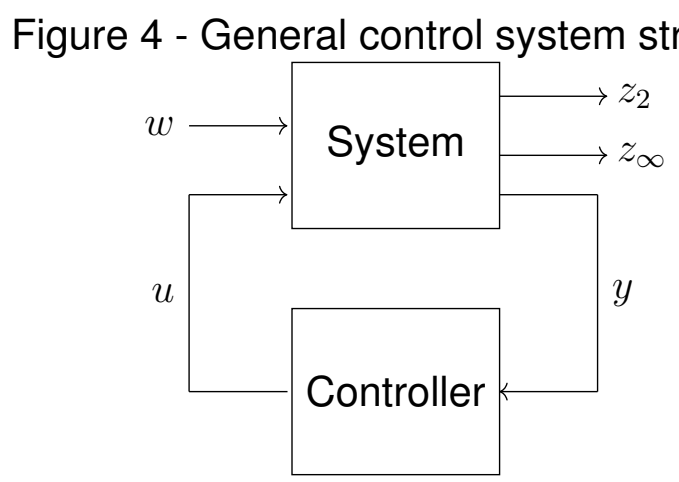

Source: Author.

The contributions of this work can be categorized in three main fronts:

- Control problems: We consider controller structures that switch according only to the observed variable, that can be viewed either as the output of a simple FDI device, or a model for a generic asynchronous behavior. We propose design conditions for state-feedback, static and dynamic output feedback controllers. Some highlights are a new formulation for the mixed $\mathcal{H}_{2} / \mathcal{H}_{\infty}$ state-feedback control for MJLS and a type of ad hoc separation procedure for the $\mathcal{H}_{2}$ and $\mathcal{H}_{\infty}$ dynamic output feedback control for hidden MJLS inspired in (OLIVEIRA; GEROMEL; BERNUSSOU, 2000). Due to the properties of our formulation, all the results encompasses the mode-dependent and cluster cases.

- Filtering problem: We derived new design conditions for $\mathcal{H}_{2}, \mathcal{H}_{\infty}$, or mixed $\mathcal{H}_{2} / \mathcal{H}_{\infty}$ filters depending only on the observed output. The Bernoulli case is also tackled, leading to necessary and sufficient results.

- Academic applications: Besides the academic applications for hidden MJLS in the context of AFTCS and NCS, we also consider a new model called $(\eta, \delta)$ SATI for wireless NCS, that was introduced in (VARMA et al., 2017). The idea is presenting an alternative to the so-called maximum allowable transmission intervals (MATI) for stochastic NCS, that can also be applied to schemes of energy efficiency in the network. 
This thesis is organized as follows. Chapter 2 presents a literature compilation concerning MJLS and observation models for the Markov chain. Chapter 3 introduces the framework used in this thesis based on (COSTA; FRAGOSO; TODOROV, 2015), along with the definitions of stochastic stability, as well as the $\mathcal{H}_{2}$ and $\mathcal{H}_{\infty}$ norms for MJLS. Chapter 4 is concerned with the state-feedback control for hidden MJLS, and Chapter 5, with the filtering problem. Chapter 6 presents a formulation for the static output feedback control and in Chapter 7 , we study the challenging problem of dynamic output feedback control in the partial observation context of the Markov chain. In Chapter 8, we consider the academic applications in AFTCS and NCS, along with the SATI formulation and co-design results for wireless NCS (WNCS). We conclude this work with our final remarks and future directions in Chapter 9. 


\section{LITERATURE REVIEW}

In this chapter, we present a literature review concerning the MJLS theory and the cases of "partial information" of the Markov chain that can be found in the literature. For a historic and deeper perspective on MJLS, we refer the reader to (MARITON, 1990; COSTA; FRAGOSO; MARQUES, 2005; BOUKAS, 2006b; COSTA; FRAGOSO; TODOROV, 2013; DRAGAN; MOROZAN; STOICA, 2013), and the references therein.

For illustrating the discussions, we define the following MJLS on the fundamental probability space $\left(\Omega, \mathfrak{F}\right.$, Prob) with filtration $\left\{\mathfrak{F}_{k}\right\}$

$$
\mathcal{G}:\left\{\begin{aligned}
x(k+1) & =A_{\theta(k)} x(k)+B_{\theta(k)} u(k)+J_{\theta(k)} w(k) \\
y(k) & =L_{\theta(k)} x(k)+H_{\theta(k)} w(k) \\
z(k) & =C_{\theta(k)} x(k)+D_{\theta(k)} u(k)+E_{\theta(k)} w(k) \\
x(0) & =x_{0}, \theta(0)=\theta_{0},
\end{aligned}\right.
$$

where $\theta(k)$ is a Markov chain taking its values on a finite set $\mathbb{N} \triangleq\{1,2, \ldots, N\}$ for $N>0$, that is, $\operatorname{Prob}\left(\theta(k+1)=j \mid \mathfrak{F}_{k}\right)=\operatorname{Prob}(\theta(k+1)=j \mid \theta(k))=p_{\theta(k) j} \geq 0$, for $j \in \mathbb{N}$, with initial distribution $\theta_{0} \sim \mu$, that is, $\operatorname{Prob}\left(\theta_{0}=i\right)=\mu_{i}$, and transition probability matrix $\mathbb{P} \triangleq\left[p_{i j}\right]$. Besides, $x$ is the state, $u$ is the control signal, $w$ is the exogenous signal, $y$ is the measured output signal, and $z$ is the controlled output signal. The concept of "partial information" of the state variables $(x, \theta)$ is concerned with which variables we could "measure" for implementing controllers and/or filters for (2.1). Inspired in (COSTA; FRAGOSO; MARQUES, 2005; COSTA; FRAGOSO; TODOROV, 2015), we group the cases of partial information on $(x, \theta)$ in the following convention,

(i) the complete observation case: both the state $x$ and the Markov chain $\theta$ can be perfectly measured;

(ii) the case of partial observation on $\theta$;

(iii) the case of partial observation on both $x$ and $\theta$.

Alternatively, we can also consider the situation in which $x$ cannot be measured, but we still have access to $\theta$. That introduces the concept of mode-dependent controllers and 
filters. Inside the realm of $(i i)$, a key idea that arose in the literature was the introduction of observation structures of $\theta$. The setting in which the controller/filter has no access at all to $\theta$ is called the mode-independent case, and an even more generalizing setting is called the cluster case. Both approaches can be considered the most important ones in the literature, see, for instance, (COSTA; FRAGOSO; TODOROV, 2015; TODOROV; FRAGOSO; COSTA, 2018), since the former case leads to non-switching, and arguably easier-to-implement structures, whereas the latter case encompasses both the modedependent and mode-independent settings. The use of alternative approaches in (ii) is of special interest to this work, as we are going to see in the next sections.

\subsection{The complete observation case}

In this setting, we assume that $x$ and $\theta$ are available to the controller, and thus $L_{\theta(k)}=I$ and $H_{\theta(k)}=0$ in (2.1). The control law here is taken in the state-feedback form $u(k)=K_{\theta(k)} x(k)$ and the goal is to design $K_{\theta(k)}$ that stabilizes the closed-loop system in some stochastic sense and minimizes some performance index. Some earlier works that dealt with this type of problem for the continuous-time formulation (also with the MJLS theory in general) are (FLORENTIN, 1961; SWORDER, 1969; WONHAM, 1970), where the last two papers dealt with the jump quadratic regulator problem (JQR), that is, $w=0$ and $x_{0}$ is a given or random initial condition with known first and second moment distributions, in the finite and infinite horizon settings. The finite horizon, discrete-time version, of the JQR was considered in (BLAIR; SWORDER, 1975), the infinite-time JQR, in (MOROZAN, 1983; CHIZECK; WILLSKY; CASTANON, 1986), and the study of controllability and observability for MJLS, and their relationship with the jump linear quadratic (JLQ) problem, in (JI; CHIZECK, 1988). The observability and detectability of continuous and discrete-time MJLS were studied in (COSTA; VAL, 2002a) and (COSTA; VAL, 2001), respectivelly, as well as the concept of weak detectability and the JLQ control for discrete-time systems, in (COSTA; VAL, 2002b). The case in which $w$ is taken as a wide sense white-noise sequence is considered in (FRAGOSO, 1989). In (COSTA; FRAGOSO; MARQUES, 2005) this problem is studied by considering that the transition probabilities along with the system matrices vary over the time. The tool of dynamic programming is used in the previous works, where the controller is obtained via coupled algebraic (differential/difference) Riccati equations. 
A related problem that generalizes the $L Q$ control for the case without jumps is the so-called $\mathcal{H}_{2}$ control. In this case, $w$ is taken either as an impulsive input, or a wide sense white-noise sequence. The $\mathcal{H}_{2}$ state-feedback control was studied in (COSTA; VAL; GEROMEL, 1997) through convex techniques, that allowed also for tackling cases where the transition matrix is uncertain, and also the design of modeindependent controllers (this concept was already given in (RAMI; GHAOUI, 1994) and (GHAOUI; RAMI, 1996)). The link between the stability operators $\mathcal{T}, \mathcal{L}, \mathcal{V}, \mathcal{J}$ of (COSTA; FRAGOSO; MARQUES, 2005) and the $\mathcal{H}_{2}$ state-feedback control was established in (TODOROV; FRAGOSO, 2016).

Furthermore, the interest in the $\mathcal{H}_{\infty}$ control that arose in the 1980s and culminated with the state-space solution presented in (DOYLE et al., 1989) for the case without jumps can also be seen in the MJLS theory in the works (SOUZA; FRAGOSO, 1993) and (FRAGOSO; VAL; JR., 1995), that tackled the discrete-time $\mathcal{H}_{\infty}$ state-feedback control in the finite- and infinite-horizon setting. The result is given in (difference or algebraic) coupled Riccati-like equations. The work (COSTA; VAL, 1996) extended the results of (FRAGOSO; VAL; JR., 1995) for a Markov chain taking its values on a countably infinite set. In terms of linear matrix inequalities (LMIs), the work (BOUKAS; SHI, 1997) presented a sufficient version of a bounded-real lemma and provided design conditions for $\mathcal{H}_{\infty}$ state-feedback controllers. The work (SEILER; SENGUPTA, 2003) provided a necessary and sufficient bounded-real lemma with the additional assumption that (2.1) is weakly controllable in order to have the necessity aspect of the result. By means of the conditions in (SEILER; SENGUPTA, 2003), the work (GONÇALVES; FIORAVANTI; GEROMEL, 2012) provided necessary and sufficient design LMIs for the $\mathcal{H}_{\infty}$ state-feedback control. It is also noteworthy that another necessary and sufficient version of a bounded-real lemma for MJLS can be found in (DRAGAN; MOROZAN; STOICA, 2013), where a simpler assumption for the necessity is used, namely that the transition probability matrix is nondegenerate. Robustness properties, that motivate the study of this class of problems, are concerned with minimizing the effects of worst-case $l_{2}$ disturbances in the system, or small-gain approaches such as the ones discussed in (DRAGAN; MOROZAN; STOICA, 2013; TODOROV; FRAGOSO, 2012), and the references therein. 
On the other hand, the so-called mixed $\mathcal{H}_{2} / \mathcal{H}_{\infty}$ state-feedback control for MJLS was tackled by (COSTA; MARQUES, 1998) through a LMI approach, in which an upper bound of the $\mathcal{H}_{2}$ norm is minimized, whilst ensuring that the $\mathcal{H}_{\infty}$ norm is bounded. This framework dates back to (BERNSTEIN; HADDAD, 1989), and was also used in (GEROMEL; PERES; SOUZA, 1995) through a convex formulation, where both works tackles the case without jumps. More recently, (TODOROV; FRAGOSO, 2016) also investigated the mixed $\mathcal{H}_{2} / \mathcal{H}_{\infty}$ and robust control considering the four operators that naturally arise in the MJLS theory, see, for instance, (COSTA; FRAGOSO; MARQUES, 2005).

\subsection{Mode-dependent filtering and control}

We now consider that $x$ cannot be measured, but instead we have access only to $y$ (as in (2.1)) and $\theta$. In the filtering problem, we aim to estimate (some combination of) the states regarding a performance criterion applied to the estimation error. For the control problem, given that $y$ and $\theta$ are available, we want to design a mode-dependent controller that stabilizes the closed-loop system in a stochastic sense, and that some performance criterion is minimized/bounded.

\subsubsection{Filtering}

It is a well-known fact that the best linear estimator in the case in which $(y, \theta)$ are measured is the Kalman filter. However, as pointed out in (COSTA; SAPORTA, 2017), considering that the gains were to be computed off-line, since the parameters of the system depend on the value of the Markov chain prior to the current time instant, it is necessary to consider all the possible trajectories up to $k$. Due to this "path-dependency", the off-line pre-calculation of the filter may be computationally prohibitive, apart from the alternative of using an on-line computation. A possible solution in terms of off-line computation is considering filter structures that switch according to the Markov chain. The mode-dependent $\mathcal{H}_{2}$ filtering was studied in (COSTA; TUESTA, 2004; COSTA; FRAGOSO; MARQUES, 2005), that presented an expression for the filter matrices obtained through coupled Riccati equations for the finite and infinite horizon problem. An interesting property is that the strictly proper filter structure of 
(COSTA; TUESTA, 2004) can be recast in an observer (Luenberger-like) form, and that the relationship between the Kalman and the $\mathcal{H}_{2}$ filter can be established. Lastly, the work (FIORAVANTI; GONÇALVES; GEROMEL, 2008) presented also necessary and sufficient conditions in the LMI formulation for the $\mathcal{H}_{2}$ filtering design that allowed for proper filter structures, and considered polytopic uncertainty on the system matrices.

Regarding the $\mathcal{H}_{\infty}$ filtering, the work (SOUZA; FRAGOSO, 1997) and (SOUZA; FRAGOSO, 2003) studied the synthesis of $\mathcal{H}_{\infty}$ observers for the infinite-horizon setting, and (SOUZA; FRAGOSO, 1997) also tackled the case of uncertain transition probabilities. The paper (GONÇALVES; FIORAVANTI; GEROMEL, 2009) presented necessary and sufficient conditions for achieving an optimal $\mathcal{H}_{\infty}$ filter with a proper structure (that can be also recast in an observer form) through the LMI formulation, and allowed also for the study of polytopic uncertainty in the system matrices. More recently, the papers (GONÇALVES; GEROMEL; FIORAVANTI, 2011) and (MORAIS et al., 2018) also tackled the filtering problem considering uncertain transition probabilities, where the latter reference also studied reduced order filters.

\subsubsection{Dynamic and static output feedback control}

A study of the separation principle for discrete-time jump linear quadratic Gaussian (JLQG) control is given in (CHIZECK; JI, 1988). The works (COSTA; TUESTA, 2003; COSTA; TUESTA, 2004) discussed the $\mathcal{H}_{2}$ separation principle for MJLS in the finite and infinite horizon setting, where the strictly proper full order controller is composed by a filter and a state-feedback structure (we present a summary of the results of (COSTA; TUESTA, 2004) in the Appendix $B$ ). The result is given in terms of CAREs for the control and filtering problem. The $\mathcal{H}_{\infty}$ control was studied in (SEILER; SENGUPTA, 2005) for the Bernoulli case. The case of $\mathcal{H}_{2}$ and $\mathcal{H}_{\infty}$ full order controllers was tackled in (GEROMEL; GONÇALVES; FIORAVANTI, 2009), where necessary and sufficient design conditions are given in the LMI formulation. More recently, the design of reduced order controllers was tackled in (MORAIS et al., 2017), where a full-order controller is used as an input to obtain the reduced structures. For the problem of uncertain transition probabilities and dynamic output feedback control, we refer the reader to (ZHANG; BOUKAS, 2009; FIORAVANTI; GONÇALVES; GEROMEL, 2013a; MORAIS et al., 2016a; MORAIS et al., 2016b; MORAIS et al., 2017), and the references therein. 
Considering the difficult static output feedback control, we refer to (MARITON; BERTRAND, 1985) that proposed necessary conditions for finding a stabilizing static output feedback control that stabilizes the continuous-time MJLS whilst minimizing a quadratic cost criterion. For the $\mathcal{H}_{2}$ control, we refer to (COSTA; VAL; GEROMEL, 1997) that follows a similar formulation as presented in (GEROMEL; PERES; SOUZA, 1993) for solving the problem. The work (BOUKAS, 2006a) presented sufficient LMI conditions for the design of stabilizing controllers considering some restrictive assumptions. More recently, the works (MORAIS et al., 2014; MORAIS et al., 2016a; MORAIS et al., 2016b) tackled this type of problem by considering the two steps procedure of (PEAUCELLE; ARZELIER, 2001; MEHDI; BOUKAS; BACHELIER, 2004) for MJLS, that also allows for the design of controllers for systems subject to uncertainty on the transition probabilities.

\subsection{The case of partial observation on $\theta$}

Motivated by the fact that it is not always possible to obtain the exact value of $\theta$ during the control/filtering application, some cases of partial observation of $\theta$ were studied in the literature. The so-called mode-independent case is the most intuitive approach and considers that the controller/filter has no access to the Markov chain. The idea can be traced back to (RAMI; GHAOUI, 1994) and the references therein for continuoustime MJLS, and (GHAOUI; RAMI, 1996) for both continuous- and discrete-time MJLS, for the design of state-feedback controllers, and rely on the LMI formulation. The work (COSTA; VAL; GEROMEL, 1997) tackled the $\mathcal{H}_{2}$ state-feedback control and (SOUZA, 2006), the design of robust stabilizing state-feedback controllers through the technique of slack variables in LMI, see, for instance, (OLIVEIRA; BERNUSSOU; GEROMEL, 1999; DAAFOUZ; BERNUSSOU, 2001). A different technique is employed in the works (VARGAS; COSTA; VAL, 2012; VARGAS; COSTA; VAL, 2016), and the references therein, where necessary non-linear conditions are derived and solved with variational convergent methods for obtaining state-feedback controllers in the finite-horizon LQ control. 
The introduction of the idea of observation in clusters in (VAL; GEROMEL; GONÇALVES, 2002) was a very important result in the partial observation context. The idea is to partition the set of Markov states $\mathbb{N}$ in $\kappa$ disjoint sets by assuming that, even though it is not possible to know in which mode of operation the Markov chain is working, it is at least possible to have access to the current cluster in which the system is actually running. It is clear that this approach generalizes the mode-dependent and -independent cases. In this context, the work (VAL; GEROMEL; GONÇALVES, 2002) tackled the $\mathcal{H}_{2}$ state-feedback control, and (GONÇALVES; FIORAVANTI; GEROMEL, 2012), the $\mathcal{H}_{\infty}$ state-feedback control. For the case in which the Markov chain follows the Bernoulli assumption, optimal $\mathcal{H}_{2}$ and $\mathcal{H}_{\infty}$, cluster and mode-independent, state-feedback controllers were studied in (FIORAVANTI; GONÇALVES; GEROMEL, 2013b).

\subsubsection{Alternative observation structures on $\theta$}

Apart from the cluster and mode-independent structures, we can list some alternative observation models that can also be found in the literature. We may say that the boost for the study of these structures can be traced back to AFTCS, for providing simpler models for FDI devices that can be used by reconfigurable (switched) controllers. Some earlier works on this subject are (SWORDER; CHOU, 1986; MARITON, 1989), and the references therein, in which an attempt is made to design controller structures that do not depend explicitly on the Markov chain, but rather on a suitable "estimation" of $\theta$. The work (SRICHANDER; WALKER, 1993) considered a model in which $\theta$ repre-

sents the failure process and $\hat{\theta}$, an observed FDI output modelled by a Markov chain conditioned on $\theta$. This observation structure influenced a great deal of works such as (MAHMOUD; JIANG; ZHANG, 2003; ABERKANE et al., 2008; ABERKANE; SAUTER; PONSART, 2008; ABERKANE; PONSART; SAUTER, 2007) for continuous-time, and (ABERKANE; PONSART; SAUTER, 2008; ABERKANE, 2009; ABERKANE, 2011) for discrete-time:

$$
\operatorname{Prob}(\hat{\theta}(k+1)=v \mid \hat{\theta}(k)=l, \theta(k)=i)=\alpha_{i l}^{(v)} .
$$

The interpretation of $\alpha_{i l}^{(v)}$ can vary according to the values of $i, l, v$, such as rate of false detection, false alarm recovery rate etc, see, for instance, the paper (MARITON, 1989). 
An alternative scheme, the so-called detector approach, is the model used in this thesis. It can be traced back to the work (COSTA; FRAGOSO, 1995) and is given as follows: for the $\sigma$-fields $\hat{\mathfrak{F}}_{0}$ and $\hat{\mathfrak{F}}_{k}$ generated by $\{w(0), x(0), \theta(0)\}$ and $\{w(0), x(0), \theta(0), \hat{\theta}(0), \ldots, w(k), x(k), \theta(k)\}$, it is assumed that

$$
\operatorname{Prob}\left(\hat{\theta}(k)=l \mid \hat{\mathfrak{F}}_{k}\right)=\operatorname{Prob}(\hat{\theta}(k)=l \mid \theta(k))=\alpha_{\theta(k) l},
$$

where, in this case, the values of $\hat{\theta}(k)$ are taken from the set $\mathbb{M} \triangleq\{1,2, \ldots, M\}$, and we set $\Upsilon \triangleq\left[\alpha_{i l}\right]$. This formulation will be better detailed in Chapter 3 and was also motivated by AFTCS. Specifically in (COSTA; FRAGOSO, 1995), stability conditions were given considering bounds on the probability of correct detection. This subject was again refered to in (COSTA; FRAGOSO; MARQUES, 2005), but it was only in the recent work (COSTA; FRAGOSO; TODOROV, 2015) that more studies were performed, concerning the $\mathcal{H}_{2}$ state-feedback control. It is safe to say that (COSTA; FRAGOSO; TODOROV, 2015) brought about a revival interest in this subject, with the works in discrete-time $\mathcal{H}_{\infty}$ state-feedback control in, for instance, (TODOROV; FRAGOSO; COSTA, 2015; TODOROV; FRAGOSO; COSTA, 2018), and for continuous-time MJLS, (STADTMANN; COSTA, 2017; RODRIGUES; TODOROV; FRAGOSO, 2015). Particularly, the papers (TODOROV; FRAGOSO; COSTA, 2015; TODOROV; FRAGOSO; COSTA, 2018) derived the bounded-real lemma used in this work. An interesting application for the model in (2.3) can be found in (SILVA; BORTOLIN; COSTA, 2011; SILVA, 2012), where $\hat{\theta}(k)$-dependent state-feedback controllers are used with varying "levels of observation" in an algorithm for obtaining mode-independent state-feedback controllers for the Long Run Average Control problem. As discussed in the introduction, due to the similarity of (2.3) with the so-called HMM, specially HMC, see, for instance, (ROSS, 2010), this type of modelling can also be called hidden Markov jump linear systems, or hidden MJLS, as in (OLIVEIRA; COSTA, 2017a). Additionally, for the continuous-time formulation of (2.3), the mean square stabilizability problem was studied in (FRAGOSO; COSTA, 2004) and (COSTA; FRAGOSO; TODOROV, 2013) for the case in which $M=N$. The interesting point is that if $\operatorname{rank}(\Upsilon)=N$, then the problem of finding a mean square stabilizing controller depending on $\hat{\theta}(t)$ can be directly recast into one of finding a mode-dependent controller.

A more recent trend can also be found in the literature, the asynchronous formula- 
tion. In this case, the liaison between MJLS and AFTCS gives place to a more generic interpretation that only aims to model the asynchronous phenomena that may arise between the plant and the controller/filter. Earlier works on this theme, such as (WU et al., 2014) modeled the asynchronous behavior simply as

$$
\operatorname{Prob}(\hat{\theta}(k+1)=v \mid \hat{\theta}(k)=l)=\alpha_{l}^{(v)},
$$

that is, the observed variable $\hat{\theta}$ is taken as an independent Markov chain. Since (2.2) and (2.3) can also be seen as types of asynchronous behaviour, some works can also be found in the literature using variations of these models, such as (CHEN et al., 2016), that adopted a somewhat similar model as in (2.2); and (WU et al., 2017), that adopted the detector-based model of (COSTA, 1995; COSTA; FRAGOSO; TODOROV, 2015). Even though the interpretations of (2.3) may differ, the mathematical model is the same. Finally, another observation structure is the one given in (OGURA et al., 2018), in which the mode can be observed only at random moments.

In this work, we adopt the interpretations that (2.3) is a model for a very simple FDI device and that it can model asynchronous phenomena between the controller and the plant. We use the terminology of (OLIVEIRA; COSTA, 2017a) to generically refer to this type of system as hidden MJLS. We point out that this similar nomenclature is used in the work (OGURA et al., 2018) for a different observation model.

\subsection{The case of partial observation on both $x$ and $\theta$}

The class of problems in which we do not have access to $x$, neither to $\theta$, is the most difficult one. Generally, there is no optimal solution for the design of controllers and filters. The framework used is mainly based on the LMI formulation.

\subsubsection{Filtering}

In this type of problem, a dynamic equation for the estimation error cannot be explicitly found as in the mode-dependent case. Then a classical observer cannot be directly implemented (since we do not have access to the plant dynamics). The works on this subject generally consider a full or reduced order filter structure and assume also that the filtered system is stable in some stochastic sense. Conditions 
for mode-independent $\mathcal{H}_{\infty}$ filtering can be found in (SOUZA, 2003), and $\mathcal{H}_{2}$ and $\mathcal{H}_{\infty}$ mode-independent filters are also given in (FIORAVANTI; GONÇALVES; GEROMEL, 2008) and (GONÇALVES; FIORAVANTI; GEROMEL, 2009) by assuming some restrictions on the Markov chain (the Bernoulli case) and on the structures of the variables. The cluster case for the $\mathcal{H}_{2}$ and $\mathcal{H}_{\infty}$ filtering was considered in (GONÇALVES; FIORAVANTI; GEROMEL, 2010), and due to the generalizing properties of the cluster formulation, the results in (GONÇALVES; FIORAVANTI; GEROMEL, 2010) also yields mode-dependent and -independent filters. Finally, optimal $\mathcal{H}_{2}$ and $\mathcal{H}_{\infty}$ filters for the Bernoulli case can be found in (FIORAVANTI; GONÇALVES; GEROMEL, 2013b).

There is also an alternative formulation presented in (COSTA, 1994) that considers

the estimation of the state conditioned on the Markov chain, that is, $x(k)_{1_{\theta(k)=i}}$, instead that of $x(k)$, where the linear minimum mean square error filter (LMMSE) is obtained. This approach was used also in the work (COSTA; GUERRA, 2002b) that tackled the problem of finding an LMMSE stationary filter, as well as (COSTA; GUERRA, 2002a) that studied the similar problem as the previous reference but considering uncertainties on the system matrices. There is also the framework of the Multiple Models (MM) approach that is used, for instance, in (BAR-SHALOM; LI; KIRUBARAJAN, 2002) and the references therein. Finally, considering the vast subject of the filter theory (and smoothing) in general, from Particle Filters to the Unscented Kalman Filter, we refer the reader to (SÄRKKÄ, 2013).

\subsubsection{Dynamic and static output feedback control}

The design of dynamic output feedback controllers with partial observation on $\theta$ is still an open problem in the literature, with a few sub-optimal formulations. In (FIORAVANTI; GONÇALVES; GEROMEL, 2013a), cluster-dependent controllers can be designed if all the system matrices are equal inside a given cluster. In (MORAIS et al., 2016a) and (MORAIS et al., 2016b), the problem is rewritten into one of finding a static output feedback controller, and solved through the two steps procedure of (PEAUCELLE; ARZELIER, 2001; MEHDI; BOUKAS; BACHELIER, 2004). More recently, (MORAIS et al., 2017) considered an algorithm that designs cluster and modeindependent controllers for a given mode-dependent structure used as an input. For the modelling in (2.2) in the continuous-time formulation, the work (ABERKANE; SAUTER; 
PONSART, 2008) provided design conditions in terms of LMI, but the final controller depends also on the mode of operation. An alternative result for the continuoustime case is given in (ABERKANE; PONSART; SAUTER, 2007), that echoes the work (GEROMEL; BERNUSSOU; OLIVEIRA, 1999) in which a state-feedback controller is given for calculating the remaining controller matrices. As for the discrete-time case considering a similar observation structure as in (2.2), the work (LIU et al., 2018) presented design conditions for the $\mathcal{H}_{\infty}$ control, but apparently the controller matrices also depend on $\theta$.

For the static output feedback control, the aforementioned (MORAIS et al., 2014; MORAIS et al., 2016a; MORAIS et al., 2016b) studied the $\mathcal{H}_{2}$ and $\mathcal{H}_{\infty}$ control through the two steps procedure of (PEAUCELLE; ARZELIER, 2001; MEHDI; BOUKAS; BACHELIER, 2004), and due to the use of slack variables, cluster and modeindependent controllers can also be synthesized. For the FDI formulation used in (2.2), the works (ABERKANE et al., 2008; ABERKANE, 2009) tackled this class of problems in the continuous and discrete-time cases through the technique of ellipsoidal sets. More recently, the work (SONG et al., 2017) studied the $l_{2}-l_{\infty}$ static output feedback control in the detector approach formulation.

\subsection{Contributions of this thesis}

The contributions of this work are mainly concerned with the design of controllers and filters in the hidden MJLS formulation of (COSTA; FRAGOSO; TODOROV, 2015), briefly described in Section 2.3.1.

- In Chapter 3, we introduce the basic concepts such as hidden Markov jump linear systems, the $\mathcal{H}_{2}$ and $\mathcal{H}_{\infty}$ norms and their calculation, both for the more general case and in the Bernoulli formulation.

- In Chapter 4, we study the mixed $\mathcal{H}_{2} / \mathcal{H}_{\infty}$ state-feedback control. Alternative conditions for the $\mathcal{H}_{2}$ and $\mathcal{H}_{\infty}$ state-feedback control with respect to the ones given in (COSTA; FRAGOSO; TODOROV, 2015) and (TODOROV; FRAGOSO; COSTA, 2015; TODOROV; FRAGOSO; COSTA, 2018) are presented, with the additional feature that mixed $\mathcal{H}_{2} / \mathcal{H}_{\infty}$ controllers can also be designed. Our results become 
necessary in the complete observation case. We study the relationship with the hidden MJLS approach and the cluster case, and the robust control, considering that the transition and detection probabilities are uncertain. The results can be found in (OLIVEIRA; COSTA, 2018a), and, for interested readers, an alternative version can be found in (OLIVEIRA; COSTA, 2016a; OLIVEIRA; COSTA, 2017c), that generalizes the work (COSTA; MARQUES, 1998) to the detector approach.

- In Chapter 5, we present conditions for the design of $\mathcal{H}_{2}$ and $\mathcal{H}_{\infty}$ filters depending only on the observed variable $\hat{\theta}$ that, if jointly solved, lead also to the mixed $\mathcal{H}_{2} / \mathcal{H}_{\infty}$ filtering formulation. Our conditions become necessary for the modedependent case. The cluster and robust cases are also investigated. Additionally, we present necessary and sufficient conditions for the Bernoulli jump systems. Our results can be found in (OLIVEIRA; COSTA, 2017a) for the $\mathcal{H}_{2}$ filtering, in (OLIVEIRA; COSTA, 2016b; OLIVEIRA; COSTA, 2017b) for the $\mathcal{H}_{\infty}$ filtering, and in (OLIVEIRA; COSTA, 2018b) for the mixed $\mathcal{H}_{2} / \mathcal{H}_{\infty}$ filtering.

- In Chapter 6, we study the design of mixed $\mathcal{H}_{2} / \mathcal{H}_{\infty}$ static output feedback controllers for hidden MJLS following the two steps procedure of (PEAUCELLE; ARZELIER, 2001; MEHDI; BOUKAS; BACHELIER, 2004), as done in the works (MORAIS et al., 2014; MORAIS et al., 2016a; MORAIS et al., 2016b). We also investigate the cluster and robust cases. Our results can be found in (OLIVEIRA; COSTA; DAAFOUZ, 2018c; OLIVEIRA; COSTA; DAAFOUZ, 2018a).

- In Chapter 7, we tackle the dynamic output feedback control for hidden MJLS. Following the ideas of the works (OLIVEIRA, 1999) and (OLIVEIRA; GEROMEL; BERNUSSOU, 2000) that presented a type of ad-hoc separation procedure for the $\mathcal{H}_{2}$ control of continuous-time LTI systems, we derive sufficient conditions for the design of $\mathcal{H}_{2}$ and $\mathcal{H}_{\infty}$ dynamic output feedback controllers in terms of bilinear matrix inequalities. The ad-hoc separation procedure in this case consists of providing a stabilizing state-feedback controller in the first step to calculate the remaining controller matrices, and then the final structure is composed by both the state-feedback gains and the "filter" structure. In the mode-dependent case, the conditions become also necessary, and then by providing suitable statefeedback gains, the controllers in (COSTA; TUESTA, 2004) and (GEROMEL; 
GONÇALVES; FIORAVANTI, 2009) (adapted for strictly proper controllers) can be retrieved. We also investigate the cluster and robust cases, and consequently provide simpler forms of calculating cluster and mode-independent dynamic output feedback controllers compared to the ones given in the literature (see Section 2.4.2). A partial result considering only stabilizing controllers can be found in (OLIVEIRA; COSTA; DAAFOUZ, 2018b), and the discussion in Chapter 7 regarding the $\mathcal{H}_{2}$ control, in (OLIVEIRA; COSTA; DAAFOUZ, 2019).

- Finally, in Chapter 8 we present some applications in the context of systems subject to faults for illustrating our results. Specifically in Section 8.3, a result for WNCS (unrelated with the hidden MJLS) for the design of mode-independent state-feedback controllers is presented, taken from our work (OLIVEIRA et al., 2018).

We finalize this literature review by pointing out some important books that deal with Markov jump system. The reader may find information in (MARITON, 1990), (BOUKAS, 2006b), (COSTA; FRAGOSO; MARQUES, 2005), (COSTA; FRAGOSO; TODOROV, 2013), (DRAGAN; MOROZAN; STOICA, 2013), and the more recent (VARGAS; COSTA; VAL, 2016). 


\section{PRELIMINARIES}

In this chapter we study the basic definitions that are necessary to derive our results. We begin in Section 3.1 with the notation that is used throughout our work. In Section 3.2.1, we introduce the framework of the hidden MJLS and in Section 3.2.2, the study of stochastic stability. In Section 3.2.3, we introduce the "limit formulation" and "deterministic" definitions of the $\mathcal{H}_{2}$ norm and derive formulae for its calculation based on the so-called gramians, presenting also conditions in terms of LMI, taken from (COSTA; FRAGOSO; TODOROV, 2015) and our work (OLIVEIRA; COSTA, 2017a). The concept of the $\mathcal{H}_{\infty}$ norm is shown in Section 3.2.4, along with the bounded-real lemma for hidden MJLS, taken from (TODOROV; FRAGOSO; COSTA, 2018).

\subsection{Notation}

For $\mathbb{Y}$ and $\mathbb{X}$ real Banach spaces, we set $\mathbb{B}(\mathbb{Y}, \mathbb{X})$ as the space of bounded linear operators of $\mathbb{Y}$ into $\mathbb{X}$, and for simplicity, $\mathbb{B}(\mathbb{Y}) \triangleq \mathbb{B}(\mathbb{Y}, \mathbb{Y})$. Specifically, the real $n$ dimensional Euclidean space is denoted by $\mathbb{R}^{n}$, and the linear space of bounded $m \times$ $n$ real operators is represented by $\mathbb{B}\left(\mathbb{R}^{n}, \mathbb{R}^{m}\right)$, with $\mathbb{B}\left(\mathbb{R}^{n}\right) \triangleq \mathbb{B}\left(\mathbb{R}^{n}, \mathbb{R}^{n}\right)$. In a Hilbert Space $\mathbb{Y},\langle\cdot ; \cdot\rangle$ represents the inner product. The superscript ' indicates the transpose of a matrix, the identity operator is represented by $I$ (or by $I_{n}$, for a $n \times n$ identity matrix), the null operator, by 0 (or equivalently $0_{n \times m}$ whenever the dimensions are needed), the trace operator by $\operatorname{Tr}(\cdot)$, and the block diagonal matrix, by $\operatorname{diag}(\cdot)$. The $i$-th eigenvalue of a square matrix is represented by $\lambda_{i}(\cdot)$, and considering a symmetric matrix $S^{\prime}=S \in \mathbb{B}\left(\mathbb{R}^{n}\right)$, we have that $S \geq(>) 0$ if $\lambda_{i}(S) \geq(>) 0$ for all $i$. For $N$ and $M$ positive integers, the sets $\mathbb{N}$ and $\mathbb{M}$ are defined, respectively, by $\mathbb{N} \triangleq\{1,2,3 \ldots, N\}$ and $\mathbb{M} \triangleq\{1,2,3, \ldots, M\}$. Furthermore, the set $\mathbb{H}^{n, m}$ represents the linear space of all $N$-sequences of real matrices $V=\left(V_{1}, V_{2}, \ldots, V_{N}\right), V_{i} \in \mathbb{B}\left(\mathbb{R}^{n}, \mathbb{R}^{m}\right), i \in \mathbb{N}$, and we write, for simplicity, that $\mathbb{H}^{n} \triangleq \mathbb{H}^{n, n}$ and $\mathbb{H}^{n+} \triangleq\left\{V \in \mathbb{H}^{n} ; V_{i} \geq 0, i \in \mathbb{N}\right\}$. For $P, V \in \mathbb{H}^{n+}$, we write that $P \geq V(P>V)$ if $P_{i}-V_{i} \geq 0\left(P_{i}-V_{i}>0\right)$ for all $i \in \mathbb{N}$. For $X \in \mathbb{H}^{n, m}$ and 
$Y \in \mathbb{H}^{n, m}$, we set

$$
\langle X ; Y\rangle \triangleq \sum_{i \in \mathbb{N}} \operatorname{Tr}\left(X_{i}^{\prime} Y_{i}\right)
$$

that induces the following norm in $\mathbb{H}^{n, m}$

$$
\|X\|_{2}^{2} \triangleq \sum_{i \in \mathbb{N}} \operatorname{Tr}\left(X_{i}^{\prime} X_{i}\right)
$$

An operator $T$ is said to be positive if $T(V) \in \mathbb{B}\left(\mathbb{H}^{n+}\right)$ whenever $V \in \mathbb{H}^{n+}$. The spectral radius of $T \in \mathbb{B}\left(\mathbb{H}^{n}\right)$ is represented by $r_{\sigma}(T)$. In this case if $r_{\sigma}(T)<1$ then for any $Q \in \mathbb{H}^{n}$ there exists a unique solution $P \in \mathbb{H}^{n}$ for the system $P-T(P)=Q$, given by $P=\sum_{k=0}^{\infty} T^{k}(Q)$ and clearly if $Q \in \mathbb{H}^{n+}$, then $P \in \mathbb{H}^{n+}$.

Let $\left(\Omega, \mathfrak{F},\left\{\mathfrak{F}_{k}\right\}\right.$, Prob) with filtration $\left\{\mathfrak{F}_{k}\right\}$ be a stochastic basis, with $\mathbf{E}(\cdot)$ representing the expected value operator and $\mathbf{E}(\cdot \mid \cdot)$, the conditional expectation operator. The Hilbert space of a random sequence of $\mathfrak{F}_{k}$-measurable vectors $w(k)$ such that

$$
\|w\|_{2}^{2} \triangleq \sum_{k=0}^{\infty} \mathbf{E}\left(\|w(k)\|^{2}\right)<\infty
$$

is denoted by $l_{2}^{r}\left(\Omega, \mathfrak{F}\right.$, Prob). Finally for $A \in \mathfrak{F}, 1_{A}$ represents the indicator function of the event $A$ (if $\omega \in A$, then $1_{A}(\omega)=1$ ).

\subsection{The hidden MJLS theory}

In this section we present the hidden Markov chain (or detector) framework that is used throughout this work, taken from (COSTA; FRAGOSO; TODOROV, 2015).

\subsubsection{Hidden Markov Jump Linear Systems}

We consider the fundamental probability space $\left(\Omega, \mathfrak{F}\right.$, Prob) with filtration $\left\{\mathfrak{F}_{k}\right\}$ and define the following MJLS

$$
\mathcal{G}:\left\{\begin{aligned}
x(k+1) & =A_{\theta(k) \hat{\theta}(k)} x(k)+J_{\theta(k) \hat{\theta}(k)} w(k) \\
x(0) & =x_{0}, \theta(0)=\theta_{0}
\end{aligned}\right.
$$

where $x(k) \in \mathbb{R}^{n_{x}}$ is the state, $w(k) \in \mathbb{R}^{r}$ is the exogenous input, and $x_{0}$ is a second order random vector in $\mathbb{R}^{n_{x}}$. We have also two stochastic processes $\theta \triangleq\{\theta(k), k \geq 0\}$ 
and $\hat{\theta} \triangleq\{\hat{\theta}(k), k \geq 0\}$. The former one is a homogeneous discrete-time Markov chain that assumes values in the set $\mathbb{N}$. Letting $\mathfrak{F}_{k}$ be the $\sigma$-field generated by $\{x(0), w(0), \theta(0), \hat{\theta}(0), x(1), w(1), \theta(1), \hat{\theta}(1), \ldots, x(k), w(k), \theta(k), \hat{\theta}(k)\}$, we assume that

$$
\operatorname{Prob}\left(\theta(k+1)=j \mid \mathfrak{F}_{k}\right)=\operatorname{Prob}(\theta(k+1)=j \mid \theta(k))=p_{\theta(k) j}, \quad j \in \mathbb{N} .
$$

The transition probability matrix is represented by $\mathbb{P} \triangleq\left[p_{i j}\right],(i, j) \in \mathbb{N} \times \mathbb{N}$, and we set $\theta(0)=\theta_{0}$, where $\theta_{0}$ is a random variable with $\operatorname{Prob}\left(\theta_{0}=i\right)=\mu_{i}>0$, for all $i \in \mathbb{N}$. We assume that the signal $\theta$ is (generally) hidden, that is, we do not have access to the values of $\theta(k)$. On the other hand, the available random variable $\hat{\theta}(k)$ takes its values on the set $\mathbb{M}$. In this context, we define the $\sigma$-fields $\hat{\mathfrak{F}}_{0}$, generated by $\{x(0), w(0), \theta(0)\}$, and $\hat{\mathfrak{F}}_{k}$, by $\{x(0), w(0), \theta(0), \hat{\theta}(0), x(1), w(1), \theta(1), \hat{\theta}(1), \ldots, x(k), w(k), \theta(k)\}, k>0$, and assume that

$$
\operatorname{Prob}\left(\hat{\theta}(k)=l \mid \hat{\mathfrak{F}}_{k}\right)=\operatorname{Prob}(\hat{\theta}(k)=l \mid \theta(k))=\alpha_{\theta(k) l}, \quad l \in \mathbb{M} .
$$

Since $\alpha_{i l} \geq 0$ for all $i \in \mathbb{N}$ such that $\sum_{l \in \mathbb{M}} \alpha_{i l}=1$, we define the sets $\mathbb{M}_{i}, i \in \mathbb{N}$ as follows

$$
\mathbb{M}_{i} \triangleq\left\{l \in \mathbb{M}: \alpha_{i l}>0\right\}, \quad \bigcup_{i \in \mathbb{N}} \mathbb{M}_{i}=\mathbb{M} .
$$

We write the detection (conditional) probabilities in the matrix form $\Upsilon \triangleq\left[\alpha_{i l}\right],(i, l) \in$ $\mathbb{N} \times \mathbb{M}$. The pair $(\theta(k), \hat{\theta}(k))$ is a hidden Markov model (HMM), specifically, a hidden Markov chain (HMC), see, for instance, the book (ROSS, 2010). From now on, we call the class of systems in (3.1), as hidden Markov jump linear systems.

Example 3.1: We consider the description of the aircraft failures given in Figure 2. In this case, we have a Markov chain $\theta$ composed by three states: $\theta(k)=1$ the nominal state, $\theta(k)=2$ a faulty state with failures in the aileron, and $\theta(k)=3$ another faulty state with failures on both the aileron and rudder. We define the following transition probability matrix, taken from (COSTA; FRAGOSO; TODOROV, 2015):

$$
\mathbb{P}=\left[p_{i j}\right]=\left[\begin{array}{ccc}
0.6 & 0.4 & 0 \\
0.2 & 0.7 & 0.1 \\
0 & 0.9 & 0.1
\end{array}\right] .
$$

We have that $\operatorname{Prob}(\theta(k+1)=1 \mid \theta(k)=1)=0.6$ and $\operatorname{Prob}(\theta(k+1)=3 \mid \theta(k)=1)=0$. 
On the other hand, we consider that we do not known in which mode of operation the system is currently into, but only an estimation of $\theta(k)$ coming from a suitable FDI device and represented by $\hat{\theta}(k)$. In this case, the conditional probability matrix is given by

$$
\Upsilon=\left[\alpha_{i l}\right]=\left[\begin{array}{ccc}
1.0 & 0 & 0 \\
0 & 0.7 & 0.3 \\
0 & 0.3 & 0.7
\end{array}\right],
$$

and then we have that $\operatorname{Prob}(\hat{\theta}(k)=1 \mid \theta(k)=1)=1$, that is, the detector would know if the system is currently on the nominal mode of operation. We also have that $\operatorname{Prob}(\hat{\theta}(k)=3 \mid \theta(k)=2)=0.3$, that is, the probability of estimating a wrong mode given that $\theta(k)=2$ is 0.3 . In this context, Figure 5 shows a possible trajectory of $\theta(k)$ in black and $\hat{\theta}(k)$ in grey in function of $k$. In this hypothetical situation, the aircraft starts

Figure 5 - A possible trajectory of $\theta(k)$ (Markov chain, in black) and $\hat{\theta}(k)$ (measured variable, in grey)

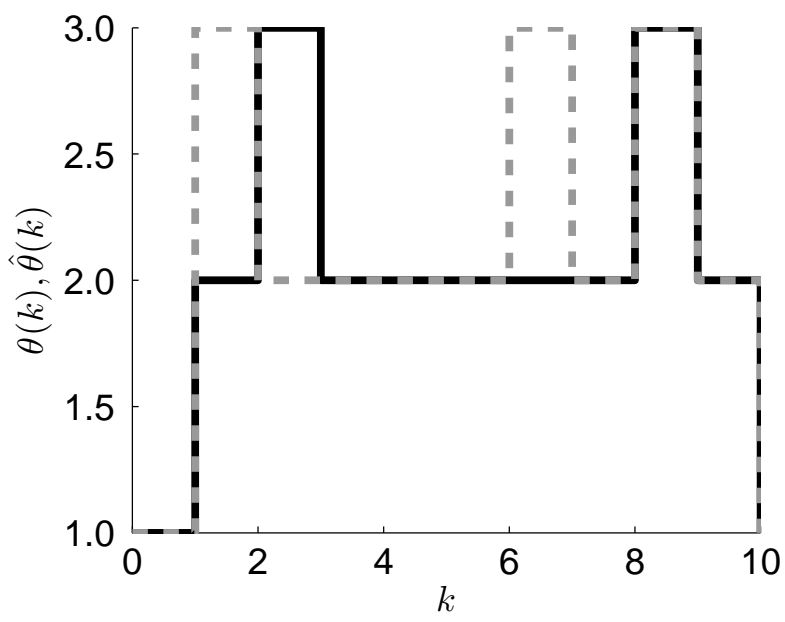

Source: Author.

working perfectly in the nominal mode of operation $\theta(k)=1$, but it promptly changes to $\theta(k)=2$, that is, the aileron does not work properly anymore. We note that in the switching process, the detector provides a wrong estimation of the mode of operation, that is, $\hat{\theta}(k)=3$. This asynchronous behavior becomes clear in Figure 5 .

Remark 3.1: An important question that may be posed is how to implement the model $(\theta(k), \hat{\theta}(k))$ in real applications. This is an open point in the literature that deserves further investigation, that is, how to satisfactorily tune the probabilities $\alpha_{i l}$. A possibility is to look at the statistics of the FDI algorithm that underlies the application as a starting 
point. In this sense, the work (MAHMOUD; JIANG; ZHANG, 2003) provides some forms of calculating the alternative formulation shown in (2.2) for the continuous-time formulation, and (CHITRAGANTI, 2014) discussed the use of semi-Markov jump linear systems and sequential change detection algorithms.

As studied in (COSTA; FRAGOSO; TODOROV, 2015), we have that the model $(\theta(k), \hat{\theta}(k))$ recovers some important cases regarding the observation of the mode $\theta(k)$ that can be found in literature.

- The mode-dependent case. This is the situation in which $\theta$ can be measured. We point out that by setting $\hat{\theta}(k)=\theta(k)$ for all $k$ we retrieve this case, that is the same to say that $\mathbb{M}=\mathbb{N}$ and $\alpha_{i i}=1$ for all $i \in \mathbb{N}$.

- The cluster case. Following (COSTA; FRAGOSO; TODOROV, 2015), there is a set $\mathbb{M}$ with $M \leq N$ such that

$$
\mathbb{N}=\bigcup_{l \in \mathbb{M}} \mathbb{N}_{l}
$$

for disjoint sets $\mathbb{N}_{l}$. Then, $c: \mathbb{N} \rightarrow \mathbb{M}$ such that $c(i)=l, i \in \mathbb{N}_{l}, c(\cdot)$ is a function that yields the cluster in which the mode $i$ is grouped. We would also have that $\mathbb{M}_{i}=\{c(i)\}$ and $\alpha_{i c(i)}=1$, and thus $\hat{\theta}(k)$ would give us the current cluster.

- The mode independent case. It can be obtained by setting $M=1$ and $\alpha_{i 1}=1$ for all $i \in \mathbb{N}$.

Remark 3.2: It is clear that there is no restriction on the numerical values of $M$ in relation to $N$ a priori. Although the cases in which $M=N$ and $M<N$ where properly characterized, the case $M>N$ could be also of interest, although this possibility is not considered in (COSTA; FRAGOSO; TODOROV, 2015).

Alternatively, there are some configurations of $\Upsilon$ that would yield the cluster and modeindependent cases, even if $M=N$. The following assumption on the partitions of $\Upsilon$ is taken from (OLIVEIRA; COSTA, 2017a).

Assumption 3.1 The cluster assumption: The modes of the Markov chain can be partitioned into $\kappa$ disjoint subsets $\mathbb{N}^{k}$, with $\bigcup_{s=1}^{\kappa} \mathbb{N}^{s}=\mathbb{N}$, such that for all $s \in\{1, \ldots, \kappa\}$, 
$i \in \mathbb{N}^{s}$, we have that $\mathbb{M}_{i}=\mathbb{M}^{s}$ for disjoint sets $\mathbb{M}^{s}, \bigcup_{s=1}^{\kappa} \mathbb{M}^{s}=\mathbb{M}$, and $\alpha_{i l}=\alpha_{l}^{s}$, for all $l \in \mathbb{M}^{s}$.

Example 3.2: Consider that $\mathbb{N}=\{1,2,3\}$ and $\mathbb{M}=\{1,2,3\}$. We define the partitions $\mathbb{N}^{1}=\{1\}$ and $\mathbb{N}^{2}=\{2,3\}$ and $\mathbb{M}_{1}=\mathbb{M}^{1}=\{1\}$ and $\mathbb{M}_{2}=\mathbb{M}_{3}=\mathbb{M}^{2}=\{2,3\}$. Additionally, assume that $\Upsilon$ has the following structure

$$
\Upsilon=\left[\begin{array}{ccc}
1 & 0 & 0 \\
0 & 1-\alpha_{2} & \alpha_{2} \\
0 & 1-\alpha_{3} & \alpha_{3}
\end{array}\right],
$$

for $0 \leq \alpha_{i} \leq 1, i \in \mathbb{M}^{2}$. This configuration respects Assumption 3.1 if $\alpha_{2}=\alpha_{3}=\alpha$.

Assumption 3.1 will allows us to establish a bridge between the hidden MJLS formulation and the cluster case in the following chapters.

Finally, we will (sometimes) consider the situation where $\mathbb{P}$ and $\Upsilon$ may not be exactly known, but are described by the following convex sets:

$$
\begin{aligned}
& \mathbb{D}_{\mathbb{P}} \triangleq\left\{\mathbb{P}: \mathbb{P}=\sum_{h=1}^{\eta} a_{h} \mathbb{P}_{h}=\sum_{h=1}^{\eta} a_{h}\left[p_{i j}^{(h)}\right], a_{h} \geq 0, \sum_{h=1}^{\eta} a_{h}=1\right\}, \\
& \mathbb{D}_{\Upsilon} \triangleq\left\{\Upsilon: \Upsilon=\sum_{t=1}^{\tau} b_{t} \Upsilon_{t}=\sum_{t=1}^{\tau} b_{t}\left[\alpha_{i l}^{(t)}\right], b_{t} \geq 0, \sum_{t=1}^{\tau} b_{t}=1\right\} .
\end{aligned}
$$

\subsubsection{Stability for hidden MJLS}

Consider the hidden MJLS (3.1) with $w=0$ defined on the probability space $\left(\Omega, \mathfrak{F}\right.$, Prob) with filtration $\left\{\mathfrak{F}_{k}\right\}$. We consider the following definition of stochastic stability taken from (COSTA; FRAGOSO; TODOROV, 2015).

Definition 3.1. System (3.1) with $w=0$ is said to be stochastically stable if, for every $\theta_{0}$ and every finite second moment $x_{0}$,

$$
\|x\|_{2}^{2}=\sum_{k=0}^{\infty} \mathbf{E}\left(\|x(k)\|^{2}\right)<\infty .
$$

In order to derive conditions for accessing the stability of (3.1), we consider the follow- 
ing operators for $V=\left(V_{1}, \ldots, V_{n}\right) \in \mathbb{H}^{n_{x}}$ :

$$
\begin{aligned}
& \mathcal{E}_{i}(V) \triangleq \sum_{j \in \mathbb{N}} p_{i j} V_{j}, \\
& \mathcal{L}_{i}(V) \triangleq \sum_{l \in \mathbb{M}_{i}} \alpha_{i l} A_{i l}^{\prime} \mathcal{E}_{i}(V) A_{i l}, \\
& \mathcal{T}_{j}(V) \triangleq \sum_{i \in \mathbb{N}} \sum_{l \in \mathbb{M}_{i}} p_{i j} \alpha_{i l} A_{i l} V_{i} A_{i l}^{\prime},
\end{aligned}
$$

for all $i, j \in \mathbb{N}$, and $\mathcal{E}, \mathcal{L}, \mathcal{T} \in \mathbb{H}^{n_{x}}$. The next theorem, adapted from (COSTA; FRAGOSO; TODOROV, 2015), provides necessary and sufficient conditions for the stochastic stability of (3.1).

Theorem 3.1. The following assertions are equivalent:

1. System (3.1) with $w=0$ is stochastically stable.

2. $r_{\sigma}(\mathcal{L})<1$ or $r_{\sigma}(\mathcal{T})<1$.

3. There exists $P \in \mathbb{H}^{n_{x}}, P>0$, such that

$$
P-\mathcal{L}(P)>0
$$

4. There exists $V \in \mathbb{H}^{n_{x}}, V>0$, such that

$$
V-\mathcal{T}(V)>0
$$

Proof. See (COSTA; FRAGOSO; TODOROV, 2015).

We now investigate the Bernoulli case, obtained if the following assumption holds.

Assumption 3.2 The Bernoulli Jump Case: For some $p_{i}>0, i \in \mathbb{N}$, we have that

$$
p_{i j}=p_{j}
$$

for all $(i, j) \in \mathbb{N} \times \mathbb{N}$.

Considering that the Bernoulli Assumption 3.2 is satisfied, we enunciate the following theorem, inspired in (FIORAVANTI et al., 2013) and (COSTA; FRAGOSO; 
TODOROV, 2015), that provides simplified conditions in respect to the ones given in Theorem 3.1 for the stochastic stability of (3.1). For that, consider the operator $\overline{\mathcal{J}} \in \mathbb{B}\left(\mathbb{R}^{n_{x}}\right)$

$$
\overline{\mathcal{J}}(\bar{V}) \triangleq \sum_{i \in \mathbb{N}} \sum_{l \in \mathbb{M}_{i}} p_{i} \alpha_{i l} A_{i l}^{\prime} \bar{V} A_{i l}
$$

for $\bar{V} \in \mathbb{B}\left(\mathbb{R}^{n_{x}}\right)$.

Theorem 3.2. Given that the Bernoulli Assumption 3.2 is satisfied, we have that $P-$ $\mathcal{L}(P)>0$ holds for $P \in \mathbb{H}^{n_{x}}, P>0$ whenever $\bar{P}-\overline{\mathcal{J}}(\bar{P})>0$ holds, for $\bar{P} \in \mathbb{B}\left(\mathbb{R}^{n_{x}}\right)$, $\bar{P}>0$.

Proof. See (COSTA; FRAGOSO; TODOROV, 2015).

We also introduce the following lemma that is necessary in the discussions of this chapter.

Lemma 3.1 (Costa, Fragoso e Marques (2005)). Let $T \in \mathbb{B}\left(\mathbb{H}^{n_{x}}\right)$ be a positive linear operator such that $r_{\sigma}(T)<1$. If $P, S \in \mathbb{H}^{n_{x}}$ are the respective solutions of $P-T(P)=Q$, $S-T(S)=V$, where $Q, V \in \mathbb{H}^{n_{x}}$ and $Q \geq V$, then $(P-S)-T(P-S)=Q-V$ and thus $P-S=\sum_{k=0}^{\infty} T^{k}(Q-V) \geq 0$, so that $P \geq S$.

\subsubsection{The $\mathcal{H}_{2}$ norm for hidden MJLS}

In this section we introduce the stochastic concept of the $\mathcal{H}_{2}$ norm of a hidden MJLS system depending on the stochastic process $(\theta(k), \hat{\theta}(k))$ as developed in (OLIVEIRA; COSTA, 2017a; COSTA; FRAGOSO; TODOROV, 2015). We derive expressions for the calculation of the $\mathcal{H}_{2}$ norm that are related to the operators that were introduced in the last section, including the Bernoulli case.

We consider the hidden MJLS (3.1) in the probability space $(\Omega, \mathfrak{F}$, Prob) with filtration $\left\{\mathfrak{F}_{k}\right\}$, and define the following controlled output,

$$
z_{2}(k)=C_{\theta(k) \hat{\theta}(k)}^{(2)} x(k)+E_{\theta(k) \hat{\theta}(k)}^{(2)} w(k),
$$

where $z_{2}(k) \in \mathbb{R}^{q_{2}}$. The state $x(k)$, the exogenous input $w(k)$, and the process $(\theta(k), \hat{\theta}(k))$ are already characterized in Section 3.2. We initially assume that: (1) $w$ 
is a wide-sense white noise sequence with identity covariance matrix (see (DAVIS; VINTER, 1985)); (2) $w(k)$ is independent of $x_{0}, \theta(k)$, and $\hat{\theta}(k)$.

In the discussion that follows we establish the relationship between the usual definition of the $\mathcal{H}_{2}$ norm, shown in Definition 3.2 below, and the so-called limit formulation of the $\mathcal{H}_{2}$ norm related to the covariance of the state $x$. For that, we need the following assumption.

Assumption 3.3: The Markov chain is ergodic (irreducible and aperiodic), see for instance, (ROSS, 2010) and (LEON-GARCIA, 2008).

Remark 3.3: We stress that Assumption 3.3 is necessary only for establishing the link between the usual definition of the $\mathcal{H}_{2}$ norm shown in Definition 3.2 and the limit formulation obtained (3.15) below. If the project goal is concerned only with Definition 3.2, Assumption 3.3 is not needed.

We have the following proposition.

Proposition 3.1: The state covariance conditioned to the $i$-th mode of operation $\hat{V}_{i}(k) \triangleq \mathbf{E}\left(x(k) x(k)^{\prime} 1_{\{\theta(k)=i\}}\right)$ is given by

$$
\hat{V}(k+1)=\mathcal{T}(\hat{V}(k))+\hat{\mathbf{J}}(k),
$$

where $\hat{V}(k) \triangleq\left(\hat{V}_{1}(k), \ldots, \hat{V}_{N}(k)\right) \in \mathbb{H}^{n_{x}+}, \hat{\mathbf{J}}(k) \triangleq\left(\hat{\mathbf{J}}_{\mathbf{1}}(k), \ldots, \hat{\mathbf{J}}_{\mathbf{N}}(k)\right) \in \mathbb{H}^{n_{x}+}, \hat{\mathbf{J}}_{\mathbf{j}}(\mathbf{k}) \triangleq$ $\sum_{i \in \mathbb{N}} \sum_{l \in \mathbb{M}_{i}} \nu_{i}(k) p_{i j} \alpha_{i l} J_{i l} J_{i l}^{\prime}$ and $\nu_{i}(k) \triangleq \operatorname{Prob}(\theta(k)=i)$.

Proof. It follows the same lines of (COSTA; FRAGOSO; MARQUES, 2005) and (OLIVEIRA; COSTA, 2017a). We evaluate

$$
\begin{array}{r}
\mathbf{E}\left(x(k+1) x(k+1)^{\prime} 1_{\{\theta(k+1)=j\}}\right)=\mathbf{E}\left(A_{\theta(k) \hat{\theta}(k)} x(k) x(k)^{\prime} A_{\theta(k) \hat{\theta}(k)}^{\prime} 1_{\{\theta(k+1)=j\}}\right) \\
+\operatorname{Her}\left[\mathbf{E}\left(J_{\theta(k) \hat{\theta}(k)} w(k) x(k)^{\prime} A_{\theta(k) \hat{\theta}(k)}^{\prime} 1_{\{\theta(k+1)=j\}}\right)\right] \\
+\mathbf{E}\left(J_{\theta(k) \hat{\theta}(k)} w(k) w(k)^{\prime} J_{\theta(k) \hat{\theta}(k)}^{\prime} 1_{\{\theta(k+1)=j\}}\right) .
\end{array}
$$


The first term is given by

$$
\begin{array}{rl}
\sum_{i \in \mathbb{N}} \sum_{l \in \mathbb{M}_{i}} & \mathbf{E}\left(A_{i l} x(k) x(k)^{\prime} A_{i l}^{\prime} 1_{\{\theta(k+1)=j\}} 1_{\{\theta(k)=i\}} 1_{\{\hat{\theta}(k)=l\}}\right) \\
= & \sum_{i \in \mathbb{N}} \sum_{l \in \mathbb{M}_{i}} A_{i l} \mathbf{E}\left(\mathbf{E}\left(x(k) x(k)^{\prime} 1_{\{\theta(k)=i\}} 1_{\{\theta(k+1)=j\}} 1_{\{\hat{\theta}(k)=l\}} \mid \mathfrak{F}_{k}\right)\right) A_{i l}^{\prime} \\
= & \sum_{i \in \mathbb{N}} \sum_{l \in \mathbb{M}_{i}} p_{i j} A_{i l} \mathbf{E}\left(\mathbf{E}\left(x(k) x(k)^{\prime} 1_{\{\theta(k)=i\}} 1_{\{\hat{\theta}(k)=l\}} \mid \hat{\mathfrak{F}}_{k}\right)\right) A_{i l}^{\prime} \\
= & \sum_{i \in \mathbb{N}} \sum_{l \in \mathbb{M}_{i}} p_{i j} \alpha_{i l} A_{i l} \hat{V}_{i}(k) A_{i l}^{\prime} .
\end{array}
$$

The second term yields to

$$
\begin{aligned}
\mathbf{E}\left(J_{\theta(k) \hat{\theta}(k)} w(k) x(k)^{\prime} A_{\theta(k) \hat{\theta}(k)}^{\prime} 1_{\{\theta(k+1)=j\}}\right) & =\sum_{i \in \mathbb{N}} p_{i j} \mathbf{E}\left(J_{i \hat{\theta}(k)} \mathbf{E}(w(k)) x(k)^{\prime} A_{i \hat{\theta}(k)}^{\prime} 1_{\{\theta(k)=i\}}\right) \\
& =0 .
\end{aligned}
$$

where the second equation comes from the independence assumptions on $w(k)$. Finally,

$$
\begin{aligned}
\mathbf{E}\left(J_{\theta(k) \hat{\theta}(k)} w(k) w(k)^{\prime} J_{\theta(k) \hat{\theta}(k)}^{\prime} 1_{\{\theta(k+1)=j\}}\right) & =\sum_{i \in \mathbb{N}} \sum_{l \in \mathbb{M}_{i}} p_{i j} \alpha_{i l} J_{i l} \mathbf{E}\left(w(k) w(k)^{\prime} 1_{\{\theta(k)=i\}}\right) J_{i l}^{\prime} \\
& =\sum_{i \in \mathbb{N}} \sum_{l \in \mathbb{M}_{i}} \nu_{i}(k) p_{i j} \alpha_{i l} J_{i l} J_{i l}^{\prime},
\end{aligned}
$$

and thus the claim follows.

By means of Assumption 3.3, and considering that (3.1) is stochastically stable, we have that $\nu(k) \rightarrow \nu$ and $\hat{V}(k) \rightarrow \hat{V}$ as $k \rightarrow \infty$. Thus, we consider this stationary situation for $\hat{V} \in \mathbb{H}^{n+}$ and $\hat{\mathbf{J}} \in \mathbb{H}^{n_{x}+}$ to write that

$$
\hat{V}=\mathcal{T}(\hat{V})+\hat{\mathbf{J}},
$$

where $\hat{\mathbf{J}}_{\mathbf{j}} \triangleq \sum_{i \in \mathbb{N}} \sum_{l \in \mathbb{M}_{i}} \nu_{i} p_{i j} \alpha_{i l} J_{i l} J_{i l}^{\prime}$. For the next proposition we also consider

$$
\hat{P}=\mathcal{L}(\hat{P})+\hat{\mathbf{C}}^{(2)},
$$

for $\hat{P} \in \mathbb{H}^{n_{x}+}, \hat{\mathbf{C}}^{(\mathbf{2})} \in \mathbb{H}^{n_{x}+}, \hat{\mathbf{C}}_{\mathbf{i}}^{(2)} \triangleq \sum_{l \in \mathbb{M}_{i}} \alpha_{i l} C_{i l}^{(2)^{\prime}} C_{i l}^{(2)}$.

Proposition 3.2: Assume that (3.1) is stochastically stable and that Assumption 3.3 
holds. We have that

$$
\begin{aligned}
\lim _{k \rightarrow \infty} \mathbf{E}\left(\left\|z_{2}(k)\right\|^{2}\right) & =\sum_{i \in \mathbb{N}} \sum_{l \in \mathbb{M}_{i}} \alpha_{i l} \operatorname{Tr}\left(C_{i l}^{(2)} \hat{V}_{i} C_{i l}^{(2)^{\prime}}+\nu_{i} E_{i l}^{(2)} E_{i l}^{(2)^{\prime}}\right) \\
& =\sum_{i \in \mathbb{N}} \sum_{l \in \mathbb{M}_{i}} \nu_{i} \alpha_{i l} \operatorname{Tr}\left(J_{i l}^{\prime} \mathcal{E}_{i}(\hat{P}) J_{i l}+E_{i l}^{(2)^{\prime}} E_{i l}^{(2)}\right)
\end{aligned}
$$

for the output (3.8), where $\hat{V}, \hat{P} \in \mathbb{H}^{n+}$ are the unique solution of (3.9) and (3.10), respectively.

Proof. Let us evaluate

$$
\begin{aligned}
\mathbf{E}\left(z_{2}(k)^{\prime} z_{2}(k)\right) & =\operatorname{Tr}\left(\mathbf{E}\left(C_{\theta(k) \hat{\theta}(k)}^{(2)} x(k) x(k)^{\prime} C_{\theta(k) \hat{\theta}(k)}^{(2)^{\prime}}\right)\right) \\
& +\operatorname{Tr}\left(\mathbf{E}\left(E_{\theta(k) \hat{\theta}(k)}^{(2)} w(k) w(k)^{\prime} E_{\theta(k) \hat{\theta}(k)}^{(2)^{\prime}}\right)\right) \\
& +\operatorname{Her}\left(\operatorname{Tr}\left(\mathbf{E}\left(E_{\theta(k) \hat{\theta}(k)}^{(2)} w(k) x(k)^{\prime} C_{\theta(k) \hat{\theta}(k)}^{(2)^{\prime}}\right)\right)\right) .
\end{aligned}
$$

The first term yields to

$$
\begin{aligned}
& \operatorname{Tr}\left(\mathbf{E}\left(C_{\theta(k) \hat{\theta}(k)}^{(2)} x(k) x(k)^{\prime} C_{\theta(k) \hat{\theta}(k)}^{(2)^{\prime}}\right)\right) \\
& =\sum_{i \in \mathbb{N}} \sum_{l \in \mathbb{M}_{i}} \operatorname{Tr}\left(C_{i l}^{(2)} \mathbf{E}\left(\mathbf{E}\left(x(k) x(k)^{\prime} 1_{\{\theta(k)=i\}} 1_{\{\hat{\theta}(k)=l\}} \mid \hat{\mathfrak{F}}_{k}\right)\right) C_{i l}^{(2)^{\prime}}\right) \\
& =\sum_{i \in \mathbb{N}} \sum_{l \in \mathbb{M}_{i}} \alpha_{i l} \operatorname{Tr}\left(C_{i l}^{(2)} \hat{V}_{i}(k) C_{i l}^{(2)^{\prime}}\right) .
\end{aligned}
$$

From the second term, we get that

$$
\begin{aligned}
\operatorname{Tr} & \left(\mathbf{E}\left(E_{\theta(k) \hat{\theta}(k)}^{(2)} w(k) w(k)^{\prime} E_{\theta(k) \hat{\theta}(k)}^{(2)^{\prime}}\right)\right) \\
& =\sum_{i \in \mathbb{N}} \sum_{l \in \mathbb{M}_{i}} \operatorname{Tr}\left(E_{i l}^{(2)} \mathbf{E}\left(\mathbf{E}\left(w(k) w(k)^{\prime} 1_{\{\theta(k)=i\}} 1_{\{\hat{\theta}(k)=l\}} \mid \hat{\mathfrak{F}}_{k}\right)\right) E_{i l}^{(2)^{\prime}}\right) \\
& =\sum_{i \in \mathbb{N}} \sum_{l \in \mathbb{M}_{i}} \alpha_{i l} \nu_{i}(k) \operatorname{Tr}\left(E_{i l}^{(2)} E_{i l}^{(2)^{\prime}}\right) .
\end{aligned}
$$

Finally, through the similar arguments given in the proof of Proposition 3.1, we have that

$$
\operatorname{Tr}\left(\mathbf{E}\left(E_{\theta(k) \hat{\theta}(k)}^{(2)} w(k) x(k)^{\prime} C_{\theta(k) \hat{\theta}(k)}^{(2)^{\prime}}\right)\right)=0 .
$$


Then, by Assumption 3.3, we have that $\hat{V}_{i}(k) \rightarrow \hat{V}_{i}$ when $k \rightarrow \infty$, leading to

$$
\lim _{k \rightarrow \infty} \mathbf{E}\left(\left\|z_{2}(k)\right\|^{2}\right)=\sum_{i \in \mathbb{N}} \sum_{l \in \mathbb{M}_{i}} \alpha_{i l} \operatorname{Tr}\left(C_{i l}^{(2)} \hat{V}_{i} C_{i l}^{(2)^{\prime}}+\nu_{i} E_{i l}^{(2)} E_{i l}^{(2)^{\prime}}\right) .
$$

Finally, we get that

$$
\begin{aligned}
\lim _{k \rightarrow \infty} \mathbf{E}\left(\left\|z_{2}(k)\right\|^{2}\right) & =\left\langle\hat{\mathbf{C}}^{(2)} ; \hat{V}\right\rangle+\sum_{i \in \mathbb{N}} \sum_{l \in \mathbb{M}_{i}} \nu_{i} \alpha_{i l} \operatorname{Tr}\left(E_{i l}^{(2)} E_{i l}^{(2)^{\prime}}\right) \\
& =\langle\hat{P}-\mathcal{L}(\hat{P}) ; \hat{V}\rangle+\sum_{i \in \mathbb{N}} \sum_{l \in \mathbb{M}_{i}} \nu_{i} \alpha_{i l} \operatorname{Tr}\left(E_{i l}^{(2)^{\prime}} E_{i l}^{(2)}\right) \\
& =\langle\hat{P} ; \hat{\mathbf{J}}\rangle+\sum_{i \in \mathbb{N}} \sum_{l \in \mathbb{M}_{i}} \nu_{i} \alpha_{i l} \operatorname{Tr}\left(E_{i l}^{(2)^{\prime}} E_{i l}^{(2)}\right) \\
& =\sum_{i \in \mathbb{N}} \sum_{l \in \mathbb{M}_{i}} \nu_{i} \alpha_{i l} \operatorname{Tr}\left(J_{i l}^{\prime} \mathcal{E}_{i}(\hat{P}) J_{i l}+E_{i l}^{(2)^{\prime}} E_{i l}^{(2)}\right)
\end{aligned}
$$

and thus, the claim follows.

We introduce the "deterministic" definition of the $\mathcal{H}_{2}$ norm in the following discussion, and its relationship with (3.11) and (3.12).

Definition 3.2 (The $\mathcal{H}_{2}$ norm for MJLS). Consider that (3.1) is stochastically stable and $x_{0}=0$. Let $z_{2 s}$ be the controlled output (3.8) for the exogenous input $w$ defined as follows:

$$
w(k)=\left\{\begin{array}{cc}
e_{s}, & k=0, \\
0, & k>0,
\end{array}\right.
$$

where $e_{s}$ is the $s$-th standard basis of $\mathbb{R}^{r}$. Then, the $\mathcal{H}_{2}$ norm of (3.1) with respect to (3.8) is defined as follows:

$$
\|\mathcal{G}\|_{2}^{2} \triangleq \sum_{s=1}^{r}\left\|z_{2 s}\right\|_{2}^{2}, \quad\left\|z_{2 s}\right\|_{2}^{2} \triangleq \sum_{k=0}^{\infty} \mathbf{E}\left(\left\|z_{2 s}(k)\right\|^{2}\right) .
$$

We point out that the definition of the $\mathcal{H}_{2}$ norm for MJLS given in Definition 3.2 becomes equivalent to the one used in the case without jumps, that is, $N=1$.

Recalling that $\mu_{i}=\operatorname{Prob}\left(\theta_{0}=i\right)$, let $V, P \in \mathbb{H}^{n_{x}}$ be the unique solution of the following equations

$$
\begin{aligned}
& V-\mathcal{T}(V)=\mathbf{J}, \\
& P-\mathcal{L}(P)=\mathbf{C}^{(\mathbf{2})},
\end{aligned}
$$


where $\mathbf{J}, \mathbf{C}^{(2)} \in \mathbb{H}^{n_{x}}$ are given by

$$
\begin{aligned}
\mathbf{J}_{j} & \triangleq \sum_{i \in \mathbb{N}} \sum_{l \in \mathbb{M}_{i}} \mu_{i} p_{i j} \alpha_{i l} J_{i l} J_{i l}^{\prime}, \\
\mathbf{C}_{\mathbf{i}}^{(\mathbf{2})} & \triangleq \sum_{l \in \mathbb{M}_{i}} \alpha_{i l} C_{i l}^{(2)^{\prime}} C_{i l}^{(2)} .
\end{aligned}
$$

Considering the definition of the $\mathcal{H}_{2}$ norm for MJLS in Definition 3.2, we can calculate the $\|\mathcal{G}\|_{2}$ through the next proposition.

Proposition 3.3: The $\mathcal{H}_{2}$ norm of (3.1) with respect to (3.8) is given by

$$
\begin{aligned}
\|\mathcal{G}\|_{2}^{2} & =\sum_{i \in \mathbb{N}} \sum_{l \in \mathbb{M}_{i}} \alpha_{i l} \operatorname{Tr}\left(C_{i l}^{(2)} V_{i} C_{i l}^{(2)^{\prime}}+\mu_{i} E_{i l}^{(2)} E_{i l}^{(2)^{\prime}}\right), \\
& =\sum_{i \in \mathbb{N}} \sum_{l \in \mathbb{M}_{i}} \mu_{i} \alpha_{i l} \operatorname{Tr}\left(J_{i l}^{\prime} \mathcal{E}_{i}(P) J_{i l}+E_{i l}^{(2)^{\prime}} E_{i l}^{(2)}\right)
\end{aligned}
$$

for $V, P$ given by (3.13) and (3.14), respectively.

Proof. See (COSTA; FRAGOSO; TODOROV, 2015).

It follows from Propositions 3.2 and 3.3 that

$$
\|\mathcal{G}\|_{2}^{2}=\lim _{k \rightarrow \infty} \mathbf{E}\left(\left\|z_{2}(k)\right\|^{2}\right)
$$

whenever $\nu=\mu$, that is, in the case that the limiting distribution $\nu$ equals the initial probability distribution $\mu$, see (COSTA; FRAGOSO; MARQUES, 2005) and (OLIVEIRA; COSTA, 2017a). We now present a form of calculating the $\mathcal{H}_{2}$ norm of (3.1) in terms of matrix inequalities. For that, we consider

$$
P-\mathcal{L}(P)>\mathbf{C}^{(2)}
$$

for $P \in \mathbb{H}^{n_{x}}, P>0$. We have the following proposition.

Proposition 3.4: If (3.16) is satisfied for some $P \in \mathbb{H}^{n_{x}}, P>0$, then (3.1) is SS and

$$
\|\mathcal{G}\|_{2}^{2}<\sum_{i \in \mathbb{N}} \sum_{l \in \mathbb{M}_{i}} \mu_{i} \alpha_{i l} \operatorname{Tr}\left(J_{i l}^{\prime} \mathcal{E}_{i}(P) J_{i l}+E_{i l}^{(2)^{\prime}} E_{i l}^{(2)}\right)<\gamma^{2}
$$

for some $\gamma>0$. Besides, for $\gamma_{\text {aux }} \triangleq \gamma^{2}$,

$$
\|\mathcal{G}\|_{2}^{2}=\inf _{\gamma, P>0}\left\{\gamma_{\text {aux }}: \text { subject to (3.16) }\right\}
$$


Proof. If follows directly from Theorem 3.1, Proposition 3.3, and Lemma 3.1.

We introduce the following matrix inequalities for a given $\gamma>0$

$$
\begin{gathered}
\sum_{i \in \mathbb{N}} \sum_{l \in \mathbb{M}_{i}} \mu_{i} \alpha_{i l} \operatorname{Tr}\left(W_{i l}\right)<\gamma^{2}, \quad\left[\begin{array}{ccc}
W_{i l} & \bullet & \bullet \\
J_{i l} & \mathcal{E}_{i}(P)^{-1} & \bullet \\
E_{i l}^{(2)} & 0 & I
\end{array}\right]>0, \\
P_{i}>\sum_{l \in \mathbb{M}_{i}} \alpha_{i l} M_{i l}^{(2)}, \quad\left[\begin{array}{ccc}
M_{i l}^{(2)} & \bullet & \bullet \\
A_{i l} & \mathcal{E}_{i}(P)^{-1} & \bullet \\
C_{i l}^{(2)} & 0 & I
\end{array}\right]>0,
\end{gathered}
$$

for all $l \in \mathbb{M}_{i}, i \in \mathbb{N}$. It is easy to verify by the Schur complement (see Lemma A.1 in the Appendix A) that (3.19) retrieves (3.17) and (3.20) yields (3.16), respectively. Then (3.18) can be rewritten as follows:

$$
\|\mathcal{G}\|_{2}^{2}=\inf _{\gamma_{\text {aux }}, P>0}\left\{\gamma_{\text {aux }}: \text { subject to }(3.19)-(3.20)\right\}
$$

for $\gamma_{a u x}=\gamma^{2}$.

We tackle now the Bernoulli case and consider that the Bernoulli Assumption 3.2 holds. For that, we consider the following inequality

$$
\bar{P}-\sum_{i \in \mathbb{N}} \sum_{l \in \mathbb{M}_{i}} p_{i} \alpha_{i l}\left(A_{i l}^{\prime} \bar{P} A_{i l}+C_{i l}^{(2)^{\prime}} C_{i l}^{(2)}\right)>0
$$

for $\bar{P} \in \mathbb{B}\left(\mathbb{R}^{n_{x}}\right), \bar{P}>0$.

Proposition 3.5: The following problems are equivalent if the Bernoulli Assumption 3.2 holds:

1. $\|\mathcal{G}\|_{2}^{2}=\inf _{P>0}\left\{\sum_{i \in \mathbb{N}} \sum_{l \in \mathbb{M}_{i}} \mu_{i} \alpha_{i l} \operatorname{Tr}\left(J_{i l}^{\prime} \mathcal{E}_{i}(P) J_{i l}+E_{i l}^{(2)^{\prime}} E_{i l}^{(2)}\right)\right.$ subject to (3.16) $\}$;

2. $\|\mathcal{G}\|_{2}^{2}=\inf _{\bar{P}>0}\left\{\sum_{i \in \mathbb{N}} \sum_{l \in \mathbb{M}_{i}} \mu_{i} \alpha_{i l} \operatorname{Tr}\left(J_{i l}^{\prime} \bar{P} J_{i l}+E_{i l}^{(2)^{\prime}} E_{i l}^{(2)}\right)\right.$ subject to (3.22) $\}$.

Proof. It follows by performing the similar steps as in (COSTA; FRAGOSO; TODOROV, 2015). 


\subsubsection{The $\mathcal{H}_{\infty}$ norm for hidden MJLS}

In this section we study the $\mathcal{H}_{\infty}$ norm for hidden MJLS. The motivation is that it provides us the worst-case input-to-output cost considering a stochastic exogenous input with finite norm. The bounded-real lemma given in this subsection is taken from (TODOROV; FRAGOSO; COSTA, 2015; TODOROV; FRAGOSO; COSTA, 2018).

We consider the hidden MJLS in (3.1) in the probability space $(\Omega, \mathfrak{F}$, Prob) with filtration $\left\{\mathfrak{F}_{k}\right\}$. We now assume that $w=\{w(k), k=0, \ldots\} \in l_{2}^{r}(\Omega, \mathfrak{F}$, Prob $)$ and define the following controlled output

$$
z_{\infty}(k)=C_{\theta(k)}^{(\infty)} x(k)+E_{\theta(k)}^{(\infty)} w(k),
$$

where $z_{\infty}(k) \in \mathbb{R}^{q_{\infty}}$.

Definition 3.3 (The $\mathcal{H}_{\infty}$ norm for MJLS). Consider that (3.1) is stochastically stable and $x_{0}=0$. The $\mathcal{H}_{\infty}$ norm of (3.1) is given by

$$
\|\mathcal{G}\|_{\infty}=\sup _{\theta_{0}, 0 \neq w \in l_{2}^{r}(\Omega, \mathfrak{F}, \operatorname{Prob})} \frac{\left\|z_{\infty}\right\|_{2}}{\|w\|_{2}}
$$

where $\left\|z_{\infty}\right\|_{2}^{2} \triangleq \sum_{k=0}^{\infty} \mathbf{E}\left(\left\|z_{\infty}(k)\right\|^{2}\right)$, for $z_{\infty}(k)$ defined in (3.23).

We introduce the following assumption that performs two roles: (1) if fulfilled, then the bounded-real lemma stated below becomes also necessary; (2) it is needed for the stability result stated in Theorem 4.1, Chapter 4.

Assumption 3.4: $\mathbb{P}$ is nondegenerate, i.e., $\sum_{i \in \mathbb{N}} p_{i j}>0, \forall j \in \mathbb{N}$.

The following result is known as the bounded-real lemma for hidden MJLS (or detector approach), taken from (TODOROV; FRAGOSO; COSTA, 2018).

Theorem 3.3 (Bounded-real lemma, (TODOROV; FRAGOSO; COSTA, 2018)). For a given $\delta>0$, consider the following statements:

(i) System (3.1) is stochastically stable with $\|\mathcal{G}\|_{\infty}<\delta$.

(ii) The following matrix inequalities hold

$$
\left[\begin{array}{cc}
Q_{i} & 0 \\
0 & \delta^{2} I
\end{array}\right]>\sum_{l \in \mathbb{M}_{i}} \alpha_{i l}\left[\begin{array}{cc}
A_{i l} & J_{i l} \\
C_{i l}^{(\infty)} & E_{i l}^{(\infty)}
\end{array}\right]^{\prime}\left[\begin{array}{cc}
\mathcal{E}_{i}(Q) & 0 \\
0 & I
\end{array}\right]\left[\begin{array}{cc}
A_{i l} & J_{i l} \\
C_{i l}^{(\infty)} & E_{i l}^{(\infty)}
\end{array}\right],
$$


for all $i \in \mathbb{N}$, and $Q \in \mathbb{H}^{n_{x}}, Q>0$.

Then $(i i) \Longrightarrow($ i) and $(i) \Longrightarrow$ (ii) if Assumption 3.4 holds.

Proof. See (TODOROV; FRAGOSO; COSTA, 2018).

Equation (3.25) can be equivalently written by means of the Schur complement and auxiliary matrices in the form

$$
\left[\begin{array}{cc}
Q_{i} & \bullet \\
0 & \delta^{2} I
\end{array}\right]>\sum_{l \in \mathbb{M}_{i}} \alpha_{i l}\left[\begin{array}{cc}
M_{i l}^{(\infty)} & \bullet \\
N_{i l}^{(\infty)} & S_{i l}^{(\infty)}
\end{array}\right], \quad\left[\begin{array}{cccc}
M_{i l}^{(\infty)} & \bullet & \bullet & \bullet \\
N_{i l}^{(\infty)} & S_{i l}^{(\infty)} & \bullet & \bullet \\
A_{i l} & J_{i l} & \mathcal{E}_{i}(Q)^{-1} & \bullet \\
C_{i l}^{(\infty)} & E_{i l}^{(\infty)} & 0 & I
\end{array}\right]>0
$$

for all $i \in \mathbb{N}, l \in \mathbb{M}_{i}$, that is a more suitable form for achieving the design conditions throughout this work. Moreover, the following corollary presents alternative, and yet more conservative, conditions for the calculation of an upper bound of the $\mathcal{H}_{\infty}$ control problem.

Corollary 3.1. System (3.1) is stochastically stable with $\|\mathcal{G}\|_{\infty}<\delta$ for a given $\delta>0$ if the following inequality set holds for all $i \in \mathbb{N}, l \in \mathbb{M}_{i}$,

$$
\begin{aligned}
& Q_{i}>\sum_{l \in \mathbb{M}_{i}} \alpha_{i l} M_{i l}^{(\infty)} \\
& {\left[\begin{array}{cc}
M_{i l}^{(\infty)} & 0 \\
0 & \delta^{2} I
\end{array}\right]>\left[\begin{array}{cc}
A_{i l} & J_{i l} \\
C_{i l}^{(\infty)} & E_{i l}^{(\infty)}
\end{array}\right]^{\prime}\left[\begin{array}{cc}
\mathcal{E}_{i}(Q) & 0 \\
0 & I
\end{array}\right]\left[\begin{array}{cc}
A_{i l} & J_{i l} \\
C_{i l}^{(\infty)} & E_{i l}^{(\infty)}
\end{array}\right], }
\end{aligned}
$$

for $Q \in \mathbb{H}^{n_{x}}, Q>0$.

Proof. It readily follows by multiplying (3.28) by $\alpha_{i l}$, summing everything up for all $l \in$ $\mathbb{M}_{i}$, recalling that $\sum_{l \in \mathbb{M}_{i}} \alpha_{i l}=1$, and considering (3.27).

In the sequence we focus our efforts in the Bernoulli case and consider that the 
Bernoulli Assumption 3.2 holds. Consider the following inequality set:

$$
\begin{gathered}
\bar{Q}>\sum_{i} p_{i} \bar{G}_{i} \\
{\left[\begin{array}{cc}
\bar{G}_{i} & \bullet \\
0 & \delta^{2} I
\end{array}\right]>\sum_{l \in \mathbb{M}_{i}} \alpha_{i l}\left[\begin{array}{cc}
A_{i l} & J_{i l} \\
C_{i l}^{(\infty)} & E_{i l}^{(\infty)}
\end{array}\right]^{\prime}\left[\begin{array}{cc}
\bar{Q} & \bullet \\
0 & I
\end{array}\right]\left[\begin{array}{cc}
A_{i l} & J_{i l} \\
C_{i l}^{(\infty)} & E_{i l}^{(\infty)}
\end{array}\right],}
\end{gathered}
$$

for all $i \in \mathbb{N}$.

Proposition 3.6: The following statements are equivalent for some given $\delta>0$ considering that the Bernoulli Assumption 3.2 holds.

1. Condition (3.25) holds and $Q \in \mathbb{H}^{n_{x}}, Q>0$.

2. Conditions (3.29)-(3.30) holds, $\bar{Q} \in \mathbb{B}\left(\mathbb{R}^{n_{x}}\right), \bar{Q}>0$ and $\bar{G} \in \mathbb{H}^{n_{x}}, \bar{G}>0$.

Proof. See (OLIVEIRA; COSTA, 2017b).

Finally, we discuss a property of the bounded-real lemma (3.25) that builds a bridge between the $\mathcal{H}_{2}$ and $\mathcal{H}_{\infty}$ theory in the next corollary.

Corollary 3.2. Consider that $C_{i l}=C_{i l}^{(2)}=C_{i l}^{(\infty)}$ and $E_{i l}=E_{i l}^{(2)}=E_{i l}^{(\infty)}$ for all $i \in \mathbb{N}, l \in$ $\mathbb{M}_{i}$. If (3.25) holds for some $\delta>0$ and $Q \in \mathbb{H}^{n_{x}}, Q>0$, then

$$
\|\mathcal{G}\|_{2}^{2}<\sum_{i \in \mathbb{N}} \sum_{l \in \mathbb{M}_{i}} \mu_{i} \alpha_{i l} \operatorname{Tr}\left(J_{i l}^{\prime} \mathcal{E}_{i}(Q) J_{i l}+E_{i l}{ }^{\prime} E_{i l}\right) .
$$

Proof. It follows by noting that if (3.25) holds, then $Q_{i}-$ $\sum_{l \in \mathbb{M}_{i}} \alpha_{i l}\left(A_{i l}^{\prime} \mathcal{E}_{i}(Q) A_{i l}+C_{i l}^{\prime} C_{i l}\right)>0$ also holds for all $i \in \mathbb{N}$. Then by Lemma 3.1, we get that $Q>S \geq 0$, where $S$ is the solution of (3.10), and then by Proposition 3.4, we get (3.31). 


\subsection{Final remarks}

The first study on the so-called detector approach can be found in (COSTA; FRAGOSO, 1995) where a bound on the probability of correct detection is obtained that ensures the stability of the closed-loop system. This discussion is also considered in (COSTA; FRAGOSO; MARQUES, 2005). More recently, the paper (COSTA; FRAGOSO; TODOROV, 2015) renovated the interest in the subject and laid the basis for this thesis. Specifically, (COSTA; FRAGOSO; TODOROV, 2015) discussed on stability conditions that resulted in Theorem 3.1, and the "deterministic" version of the $\mathcal{H}_{2}$ norm (and state-feedback control). The "limit form" and a more general version of the $\mathcal{H}_{2}$ norm can be found in (OLIVEIRA; COSTA, 2017a), that considered the "feedthrough" term $E_{\theta(k)}^{(2)}$. The bounded-real lemma for the detector approach (hidden MJLS) first appeared in (TODOROV; FRAGOSO; COSTA, 2015) in its sufficient form, whereas (TODOROV; FRAGOSO; COSTA, 2018) presented the also necessity part. Bernoulli conditions for the $\mathcal{H}_{\infty}$ norm can be found in (TODOROV; FRAGOSO; COSTA, 2018; OLIVEIRA; COSTA, 2017b). The fact that the bounded-real lemma for the detector approach also provides a bound on the $\mathcal{H}_{2}$ norm as presented in Corollary 3.2 is discussed in (OLIVEIRA; COSTA, 2018a), but is not a new idea. We refer the interested reader to (BERNSTEIN; HADDAD, 1989; KHARGONEKAR; ROTEA, 1991; KHARGONEKAR; ROTEA; BAEYENS, 1996; GEROMEL; PERES; SOUZA, 1995), and the references therein. 


\section{STATE-FEEDBACK CONTROL FOR HIDDEN MJLS}

In this chapter, we study the state-feedback control in the hidden MJLS formulation, that is, the problem of finding admissible controllers depending only on $\hat{\theta}(k)$ such that the plant is stochastically stable and eventually respects one or more performance indexes. Prior to this thesis, some works had already tackled this problem, such as (COSTA; FRAGOSO; TODOROV, 2015) for the $\mathcal{H}_{2}$ control and (TODOROV; FRAGOSO; COSTA, 2015) for the $\mathcal{H}_{\infty}$ control. The novelty here consists in tackling both problems at the same time in a mixed $\mathcal{H}_{2} / \mathcal{H}_{\infty}$ control formulation, a problem that cannot be solved directly by combining the conditions given in (COSTA; FRAGOSO; TODOROV, 2015; TODOROV; FRAGOSO; COSTA, 2015). In this sense, we present new $\mathcal{H}_{2}$ and $\mathcal{H}_{\infty}$ design LMls compared to the ones given in the aforementioned works, that can also retrieve the optimal control for each case taken separately in the modedependent case.

A first study on the subject was performed in the works (OLIVEIRA; COSTA, 2016a; OLIVEIRA; COSTA, 2017c), where we derived a type of Riccaty inequality that generalizes the one given in (COSTA; MARQUES, 1998) for hidden MJLS and presented design conditions for the mixed $\mathcal{H}_{2} / \mathcal{H}_{\infty}$ control. That led to a more general formulation for the problem by considering slack variables akin to (OLIVEIRA; GEROMEL; BERNUSSOU, 2002; TODOROV; FRAGOSO, 2016), that is related to the observability gramian and the bounded-real lemma of (TODOROV; FRAGOSO; COSTA, 2015; TODOROV; FRAGOSO; COSTA, 2018) and does not require some restrictions, namely, relying on the so-called Lyapunov shaping paradigm of (SCHERER; GAHINET; CHILALI, 1997), and fixing $J_{\theta(k)}=J$ as done in (OLIVEIRA; COSTA, 2016a; OLIVEIRA; COSTA, 2017c; COSTA; MARQUES, 1998). Our results can also be found in (OLIVEIRA; COSTA; DAAFOUZ, 2018b; OLIVEIRA; COSTA, 2018a).

This chapter is organized as follows. We present the problem formulation in Section 4.1. The results for the design of stabilizing, $\mathcal{H}_{2}, \mathcal{H}_{\infty}$, and mixed $\mathcal{H}_{2} / \mathcal{H}_{\infty}$ state-feedback controllers can be found in Sections 4.2, 4.3, 4.4, and 4.5, respectively. The mixed $\mathcal{H}_{2} / \mathcal{H}_{\infty}$ control for the cluster case and robust control can be found in Section 4.6. Our final remarks are given in Section 4.7. 


\subsection{Problem formulation}

We define the following MJLS in a probability space $\left(\Omega, \mathfrak{F}\right.$, Prob) with filtration $\left\{\mathfrak{F}_{k}\right\}$ :

$$
\mathcal{G}:\left\{\begin{aligned}
x(k+1) & =A_{\theta(k)} x(k)+B_{\theta(k)} u(k)+J_{\theta(k)} w(k) \\
z_{2}(k) & =C_{\theta(k)}^{(2)} x(k)+D_{\theta(k)}^{(2)} u(k)+E_{\theta(k)}^{(2)} w(k) \\
z_{\infty}(k) & =C_{\theta(k)}^{(\infty)} x(k)+D_{\theta(k)}^{(\infty)} u(k)+E_{\theta(k)}^{(\infty)} w(k) \\
x(0) & =x_{0}, \theta_{0} \sim \mu
\end{aligned}\right.
$$

where $x(k) \in \mathbb{R}^{n}$ is the state, $u(k) \in \mathbb{R}^{m}$ is the control input, $w(k) \in \mathbb{R}^{r}$ is the exogenous input, $z_{2}(k) \in \mathbb{R}^{p_{2}}$ and $z_{\infty}(k) \in \mathbb{R}^{p_{\infty}}$ are the controlled outputs related to the $\mathcal{H}_{2}$ and $\mathcal{H}_{\infty}$ control, respectively. We adopt the control law given by

$$
u(k)=K_{\hat{\theta}(k)} x(k)
$$

and connect (4.2) to (4.1) in order to write the closed-loop system

$$
\mathcal{G}_{K}:\left\{\begin{aligned}
x(k+1) & =A_{\theta(k) \hat{\theta}(k)} x(k)+J_{\theta(k)} w(k) \\
z_{2}(k) & =C_{\theta(k) \hat{\theta}(k)}^{(2)} x(k)+E_{\theta(k)}^{(2)} w(k) \\
z_{\infty}(k) & =C_{\theta(k) \hat{\theta}(k)}^{(\infty)} x(k)+E_{\theta(k)}^{(\infty)} w(k) \\
x(0) & =x_{0}, \theta_{0} \sim \mu,
\end{aligned}\right.
$$

where $A_{\theta(k) \hat{\theta}(k)}, C_{\theta(k) \hat{\theta}(k)}^{(2)}$ and $C_{\theta(k) \hat{\theta}(k)}^{(\infty)}$ are given by

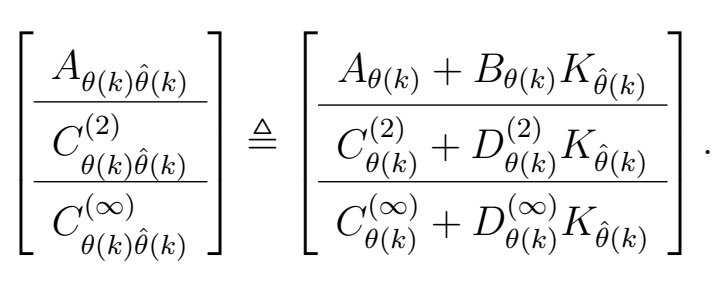

The output $z_{2}$ refers to the $\mathcal{H}_{2}$ norm presented in Definition 3.2 and $z_{\infty}$, to the $\mathcal{H}_{\infty}$ norm in Definition 3.3. Then, the exogenous input could be impulsive as in Definition 3.2 (or a wide-sense white noise sequence if only the $\mathcal{H}_{2}$ control is considered), or some stochastic sequence $w \in l_{2}^{r}(\Omega, \mathfrak{F}$, Prob), $w \neq 0$, as used in Definition 3.3. The initial condition $x_{0}$ can be taken deterministically, that is, $x_{0}=0$, or be a second order random variable in the "limit form" of the $\mathcal{H}_{2}$ control. 
The set of all admissible controllers is, for $A \triangleq\left(A_{1}, \ldots, A_{N}\right), B \triangleq\left(B_{1}, \ldots, B_{N}\right)$, and $K \triangleq\left(K_{1}, \ldots, K_{M}\right)$, represented by

$$
\mathbb{K} \triangleq\{K: \text { stochastically stabilizes }(A, B)\},
$$

or equivalently, $K$ that stochasticallly stabilizes (4.3).

In the following, we study the design of admissible feedback controllers considering upper bounds on the $\mathcal{H}_{2}, \mathcal{H}_{\infty}$ and mixed $\mathcal{H}_{2} / \mathcal{H}_{\infty}$ norms as performances indexes (measures), see, for instance, (KHARGONEKAR; ROTEA, 1991). For that, we introduce informally the problem of interest,

$$
\inf _{K \in \mathbb{K}, \gamma>0, \delta>0}\left\{g(\gamma, \delta) \text {; such that }\left\|\mathcal{G}_{K}\right\|_{2}<\gamma \text { and }\left\|\mathcal{G}_{K}\right\|_{\infty}<\delta\right\},
$$

where $\left\|\mathcal{G}_{K}\right\|_{2}$ is defined with respect to the output $z_{2}$ (see Definition 3.2) and $\left\|\mathcal{G}_{K}\right\|_{\infty}$, to $z_{\infty}$ (see Definition 3.3). The objective function $g(\gamma, \delta)$ can be chosen among the following options ${ }^{1}$ :

Control Project 1 (The mixed $\mathcal{H}_{2} / \mathcal{H}_{\infty}$ control problem). Find $K \in \mathbb{K}$ such that a suitable upper bound for the $\mathcal{H}_{2}$ norm of the closed-loop system (4.3) is obtained, subject to a given upper bound on the $\mathcal{H}_{\infty}$ norm. In this case, for a given $\delta>0$, we set

$$
g(\gamma, \delta)=\gamma^{2}
$$

Control Project 2 (The mixed "inverse" $\mathcal{H}_{2} / \mathcal{H}_{\infty}$ control problem). Find $K \in \mathbb{K}$ such that a suitable upper bound for the $\mathcal{H}_{\infty}$ norm of the closed-loop system (4.3) is obtained, subject to a given upper bound on the $\mathcal{H}_{2}$ norm. For a given $\delta>0$, this is obtained by setting

$$
g(\gamma, \delta)=\delta^{2}
$$

Control Project 3 (A weighted combination of the $\mathcal{H}_{2}$ and $\mathcal{H}_{\infty}$ costs). Find $K \in \mathbb{K}$ such that weighted combinations of suitable upper bounds of both $\mathcal{H}_{2}$ and $\mathcal{H}_{\infty}$ norms of (4.3) are obtained. Thus, for given $\beta^{(2)} \geq 0$ and $\beta^{(\infty)} \geq 0$, we set

$$
g(\gamma, \delta)=\gamma^{2} \beta^{(2)}+\delta^{2} \beta^{(\infty)} .
$$

\footnotetext{
${ }^{1}$ This formulation is also described in (GAHINET et al., 1995) and implemented in the LMI Control Toolbox of MATLAB by the function hinfmix for continuous-time linear systems.
} 
A first approach for solving (4.6) is to use (3.16)-(3.17) along with (3.25) with system matrices in (4.4). In this case, problem (4.6) could

$$
\inf _{K \in \mathbb{K}, P, W, Q, \gamma, \delta}\{g(\gamma, \delta) \text { : such that (3.16) - (3.17) and (3.25) hold }\} .
$$

The optimization problem (4.7) in its current form is highly non-linear on the decision variables and thus hard to solve. The main goal of this chapter is to circumvent such difficulties by jointly linearizing conditions (3.19)-(3.20) and (3.25), thus providing a design method akin to the one given in (TODOROV; FRAGOSO, 2016). For the study that follows we use the notation:

- For a given matrix sequence $V=\left(V_{1}, \ldots, V_{M}\right), V \in \mathbb{H}^{n}, \Phi(V) \triangleq \operatorname{diag}\left(V_{1}, \ldots, V_{M}\right)$. Besides, for $V_{i l}, i \in \mathbb{N}, l \in M_{i}$, we set $\Phi_{i}(V) \triangleq \operatorname{diag}\left(V_{i 1}, \ldots, V_{i M}\right)$;

- For a given matrix with positive coefficients $T=\left[\tau_{i j}\right], T \in \mathbb{R}^{N \times M}, \tau_{i j} \geq 0$,

$$
\Gamma_{i}(T) \triangleq\left[\begin{array}{lll}
\sqrt{\tau_{i 1}} I & \cdots & \sqrt{\tau_{i M}} I
\end{array}\right]^{\prime} .
$$

\subsection{Stabilizing state-feedback control for hidden MJLS}

In this section, we briefly present an LMI design condition for $K \in \mathbb{K}$ that extends the result given in (COSTA; FRAGOSO; TODOROV, 2015) by considering an additional degree of freedom. Consider the following matrices inequalities for a given scalar $\beta$,

$$
\left[\begin{array}{cc}
R_{i l}-\beta \operatorname{Her}\left(A_{i} G_{l}+B_{i} Y_{l}\right) & A_{i} G_{l}+B_{i} Y_{l}-G_{l}^{\prime} \beta \\
\bullet & \operatorname{Her}\left(G_{l}\right)-\mathcal{D}_{i}(\tilde{R})
\end{array}\right]>0,
$$

for all $i \in \mathbb{N}, l \in \mathbb{M}_{i}$, where $\tilde{R} \triangleq\left(R_{i l} \in \mathbb{B}\left(\mathbb{R}^{n}\right) ; i \in \mathbb{N}, l \in \mathbb{M}_{i}\right)$ and $\mathcal{D}_{i}(\tilde{R}) \triangleq$ $\sum_{j \in \mathbb{N}} \sum_{l \in \mathbb{M}_{j}} p_{j i} \alpha_{j l} R_{j l}$. The next theorem is an extension of Theorem 1 of (COSTA; FRAGOSO; TODOROV, 2015).

Theorem 4.1. Consider the following statements:

(i) There exist a given scalar $\beta$ and matrices $\tilde{R}>0, G_{l}$, and $Y_{l}$ of compatible dimensions such that (4.8) holds.

(ii) There exists $K \in \mathbb{K}$. 
We have by setting $K_{l}=Y_{l} G_{l}^{-1}$ for all $l \in \mathbb{M}$ that $(i) \Longrightarrow(i i)$. Besides, if $\hat{\theta}=\theta$, then in addition $($ ii $) \Longrightarrow($ i). Besides, $-1<\beta<1$.

Proof. Set $Y_{l}=K_{l} G_{l}$ in (4.8) and $A_{i l}^{(K)} \triangleq A_{i}+B_{i} K_{l}$, in order to rewrite (4.8) as follows

$$
\left[\begin{array}{cc}
R_{i l} & 0 \\
0 & -\mathcal{D}_{i}(\tilde{R})
\end{array}\right]+\operatorname{Her}\left(\left[\begin{array}{c}
A_{i l}^{(K)} \\
I
\end{array}\right] G_{l}\left[\begin{array}{ll}
-I \beta & I
\end{array}\right]\right)>0
$$

for all $i \in \mathbb{N}, l \in \mathbb{M}_{i}$. By means of the Elimination Lemma (see Lemma A.3 in the Appendix A), we have that $U^{\prime}=\left[\begin{array}{cc}A_{i l}^{(K)^{\prime}} & I\end{array}\right]$ and $V^{\prime}=\left[\begin{array}{ll}-I \beta & I\end{array}\right]$. By setting $\tilde{U}^{\prime}=$ $\left[\begin{array}{ll}-I & A_{i l}^{(K)}\end{array}\right]$ and $\tilde{V}^{\prime}=\left[\begin{array}{ll}I & I \beta\end{array}\right]$, we get that $\tilde{U}^{\prime} U=0$ and $\tilde{V}^{\prime} V=0$, so that (4.8) holds only if $R_{i l}-A_{i l}^{(K)} \mathcal{D}_{i}(\tilde{R}) A_{i l}^{(K)^{\prime}}>0$ and $R_{i l}-\beta^{2} \mathcal{D}_{i}(\tilde{R})>0$ jointly hold for all $i \in \mathbb{N}, l \in \mathbb{M}_{i}$. Recalling Assumption 3.4, we have at least one element $p_{i j} \alpha_{i l}>0, i \in \mathbb{N}, l \in \mathbb{M}_{i}$, so that $\mathcal{D}_{i}(\tilde{R})>0$ for all $i \in \mathbb{N}$. Thus by defining $Q_{i}=\mathcal{D}_{i}(\tilde{R})$ and performing the same steps of Theorem 1 as in (COSTA; FRAGOSO; TODOROV, 2015) in the first inequality, we note that by setting $K_{l}=Y_{l} G_{l}^{-1}$, then $K \in \mathbb{K}$. For $(i) \Longrightarrow$ (ii), note that if $\hat{\theta}=\theta$, then $\alpha_{i i}=1$ for all $i \in \mathbb{N}$, it is direct that (4.8) retrieves Equation (13) of (VAL; GEROMEL; GONÇALVES, 2002) with $\beta=0$ and $E_{i}=0$ for all $i \in \mathbb{N}$. Finally, considering that $R_{i l}-\beta^{2} \mathcal{D}_{i}(\tilde{R})>0$ must hold, define $R_{i}=\sum_{l \in \mathbb{M}_{i}} \alpha_{i l} R_{i l}$, and then we have that $R_{i l}-\beta^{2} \sum_{j \in \mathbb{N}} p_{j i} R_{j}>0$. By multiplying this inequality by $\alpha_{i l}$ and summing the result up for all $l \in \mathbb{M}_{i}$ and $i \in \mathbb{N}$, we get after simple manipulations in the spirit of (MORAIS et al., 2016a) that $\left(1-\beta^{2}\right) \sum_{i \in \mathbb{N}} R_{i}>0$, and thus $-1<\beta<1$.

Remark 4.1: The main role of $\beta$ in (4.8) is to decrease the conservatism of Theorem 4.1 in the partial observation case, by allowing a line search procedure if (4.8) fails to hold, thus extending the result given in (COSTA; FRAGOSO; TODOROV, 2015). We point out that for the study of the $\mathcal{H}_{2}$ and $\mathcal{H}_{\infty}$ state-feedback control, we opted for presenting simpler conditions without the additional degree of freedom provided by $\beta$.

Example 4.1 Stabilizing state-feedback control: We take the following dynamic and controlled input matrices

$$
A_{1}=\left[\begin{array}{cc}
1.4 & 0.1 \\
0 & 0.5
\end{array}\right], A_{2}=\left[\begin{array}{cc}
0.9 & 0 \\
0.3 & 1.2
\end{array}\right], B_{1}=\left[\begin{array}{l}
2 \\
1
\end{array}\right], B_{2}=\left[\begin{array}{l}
0 \\
0
\end{array}\right],
$$


along with the following transition probability matrix

$$
\mathbb{P}=\left[\begin{array}{ll}
0.9 & 0.1 \\
0.8 & 0.2
\end{array}\right]
$$

and thus, we have that the spectral radius of the operator $\mathcal{L}$, represented by $r_{\sigma}(\mathcal{L})$ (see (COSTA; FRAGOSO; MARQUES, 2005), Chapter 3, page 35), calculated through MATLAB, is given by $=1.8460$, that is, it is a stochastically unstable MJLS. We consider the following conditional probability matrix

$$
\left[\alpha_{i l}\right]=\left[\begin{array}{cc}
\rho_{1} & 1-\rho_{1} \\
1-\rho_{2} & \rho_{2}
\end{array}\right] .
$$

Initially, we set $\rho_{1}=\rho_{2}=0.7$ and calculate the following state-feedback controllers with $\beta=0$

$$
\left[\begin{array}{l}
K_{1} \\
K_{2}
\end{array}\right]=\left[\begin{array}{ll}
-0.6063 & -0.1132 \\
\hline-0.5903 & -0.1231
\end{array}\right],
$$

with $r_{\sigma}(\mathcal{L})=0.4468<1$. Besides, we set $\beta=0.5$ in order to obtain

$$
\left[\begin{array}{l}
K_{1} \\
K_{2}
\end{array}\right]=\left[\begin{array}{ll}
-0.4560 & -0.0379 \\
\hline-0.4192 & -0.0333
\end{array}\right],
$$

with $r_{\sigma}(\mathcal{L})=0.3976$.

\subsection{The $\mathcal{H}_{2}$ state-feedback control for hidden MJLS}

We introduce the following inequalities,

$$
\begin{aligned}
& \sum_{i \in \mathbb{N}} \mu_{i} \operatorname{Tr}\left(W_{i}\right)<\gamma^{2}, \quad\left[\begin{array}{ccc}
W_{i} & \bullet & \bullet \\
\Gamma_{i}(\mathbb{P}) J_{i} & \Phi(P) & \bullet \\
E_{i}^{(2)} & 0 & I
\end{array}\right]>0, \\
& {\left[\begin{array}{cc}
P_{i} & \bullet \\
\Gamma_{i}(\Upsilon) P_{i} & \Phi_{i}(X)
\end{array}\right]>0,\left[\begin{array}{ccc}
G_{l}+G_{l}^{\prime}-X_{i l} & \bullet & \bullet \\
\Gamma_{i}(\mathbb{P})\left(A_{i} G_{l}+B_{i} Y_{l}\right) & \Phi(P) & \bullet \\
C_{i}^{(2)} G_{l}+D_{i}^{(2)} Y_{l} & 0 & I
\end{array}\right]>0,}
\end{aligned}
$$


for all $i \in \mathbb{N}, l \in \mathbb{M}_{i}$. The set of variables of (4.10)-(4.11) for a given $\gamma$ is represented by

$$
\xi_{2} \triangleq\left\{P_{i}>0, W_{i}>0, X_{i l}>0, i \in \mathbb{N}, l \in \mathbb{M}_{i}, G_{l}, Y_{l}, l \in \mathbb{M}\right\}
$$

The set of all possible solutions of (4.10)-(4.11) for a given $\gamma$ is $\Xi_{2} \triangleq$ $\left\{\xi_{2}\right.$ such that $(4.10)-(4.11)$ holds $\}$. We have the following lemma.

Lemma 4.1. Consider the following statements for a given $\gamma>0$ :

(i) There exists $\xi_{2}$ such that $\xi_{2} \in \Xi_{2}$.

(ii) There exists a state-feedback controller $K$ such that $K \in \mathbb{K}$ and $\left\|\mathcal{G}_{K}\right\|_{2}<\gamma$.

We have that $(i) \Longrightarrow$ (ii) by taking $K_{l}=Y_{l} G_{l}^{-1}$ for all $l \in \mathbb{M}$. Besides, if $\hat{\theta}=\theta$, we have that $(i i) \Longrightarrow(i)$.

Proof. Considering that $\xi_{2} \in \Xi_{2}$ and conveniently applying the Schur complement to the second inequality in (4.10) yields

$$
\left[\begin{array}{ccc}
W_{i} & \bullet & \bullet \\
J_{i} & \left(\sum_{j \in \mathbb{N}} p_{i j} P_{j}^{-1}\right)^{-1} & \bullet \\
E_{i}^{(2)} & 0 & I
\end{array}\right]>0,
$$

for all $i \in \mathbb{N}$. Since $G_{l}+G_{l}^{\prime}>X_{i l}>0$ for all $i \in \mathbb{N}, l \in \mathbb{M}_{i}$, we have that $G_{l}$ is nonsingular. Then, by applying the result of Lemma A.2 (see the Appendix A) in (4.11), setting $Y_{l}=K_{l} G_{l}$, applying the congruence transformation $\operatorname{diag}\left(G_{l}^{-1}, I, I\right)$ to the resulting inequalities, and the Schur complement, allow us to write that

$$
\left[\begin{array}{ccc}
X_{i l}^{-1} & \bullet & \bullet \\
A_{i l} & \left(\sum_{j \in \mathbb{N}} p_{i j} P_{j}^{-1}\right)^{-1} & \bullet \\
C_{i l}^{(2)} & 0 & I
\end{array}\right]>0,
$$

holds for all $i \in \mathbb{N}, l \in \mathbb{M}_{i}$, for $A_{i l}$ and $C_{i l}^{(2)}$ given by (4.4). Besides, applying the congruence transformation $\operatorname{diag}\left(P_{i}^{-1}, I\right)$ to the first inequality of (4.11) and the Schur complement yields $P_{i}^{-1}>\sum_{l \in \mathbb{M}_{i}} \alpha_{i l} X_{i l}^{-1}$. It is clear that the last inequality, along with (4.12)-(4.13) and the first inequality of (4.10) are equivalent to (3.19)-(3.20), and thus $K \in \mathbb{K}$ and $\left\|\mathcal{G}_{K}\right\|_{2}<\gamma$. 
Conversely, $\hat{\theta}=\theta$ implies that $\alpha_{i i}=1$ for all $i \in \mathbb{N}$. Then it is clear that (4.10) is equal to the $\mathcal{H}_{2}$ optimal design conditions given in (GONÇALVES, 2014) since they do not depend on $\alpha_{i l}$. Applying a suitable perturbation $\epsilon_{i}>0$ on the remaining conditions of (GONÇALVES, 2014) such that

$$
\left[\begin{array}{ccc}
G_{i}+G_{i}^{\prime}-\left(P_{i}+I \epsilon_{i}\right) & \bullet & \bullet \\
\Gamma_{i}(\mathbb{P})\left(A_{i} G_{i}+B_{i} Y_{i}\right) & \Phi(P) & \bullet \\
C_{i}^{(2)} G_{i}+D_{i}^{(2)} Y_{i} & 0 & I
\end{array}\right]>0
$$

still hold for all $i \in \mathbb{N}$, and defining $P_{i}<X_{i i}<P_{i}+I \epsilon_{i}$ allow us to get (4.11).

Considering Lemma 4.1, we can write the $\mathcal{H}_{2}$ control problem as follows

$$
\inf _{\bar{\xi}_{2} \in \bar{\Xi}_{2}} \gamma_{\text {aux }}
$$

where $\bar{\xi}_{2} \triangleq \xi_{2} \cup\left\{\gamma_{a u x}\right\}, \gamma_{a u x} \triangleq \gamma^{2}$, and $\bar{\Xi}_{2} \triangleq\left\{\bar{\xi}_{2}\right.$ such that $(4.10)-(4.11)$ holds $\}$.

Example 4.2 $\mathcal{H}_{2}$ state-feedback control: We use the system matrices of Example 4.1, along with

$$
J_{1}=\left[\begin{array}{ccc}
0.5 & 0 & 0 \\
0 & 0.4 & 0
\end{array}\right], J_{2}=\left[\begin{array}{ccc}
1.0 & 0 & 0 \\
0 & 0.8 & 0
\end{array}\right], C_{1}^{(2)}=\left[\begin{array}{cc}
1 & -1 \\
0 & 0
\end{array}\right], C_{2}^{(2)}=\left[\begin{array}{ll}
1 & 0 \\
0 & 0
\end{array}\right],
$$

and the controlled output matrices are taken as follows $D_{1}^{(2)}=D_{2}^{(2)}=\left[\begin{array}{ll}0 & 1\end{array}\right]^{\prime}$, along with $E_{1}^{(2)}=E_{2}^{(2)}=0_{2 \times 3}$. The initial distribution of the Markov chain $\mu$ is taken as the stationary distribution. We set $\rho_{1}=\rho_{2}=1$ (the mode-dependent case) and calculate the following MJLS $\mathcal{H}_{2}$ optimal state-feedback controller by (4.14)

$$
\left[\begin{array}{l}
K_{1} \\
K_{2}
\end{array}\right]=\left[\begin{array}{cc}
-0.7405 & 0.0667 \\
\hline 0 & 0
\end{array}\right]
$$

with $r_{\sigma}(\mathcal{L})=0.3983$ and the optimal cost given by $\left\|\mathcal{G}_{*}\right\|_{2}=\gamma^{*}=0.9870$. On the other hand, we set $\rho_{1}=\rho_{2}=0.7$ and calculate the following state-feedback controller through (4.14),

$$
\left[\begin{array}{l}
K_{1} \\
K_{2}
\end{array}\right]=\left[\begin{array}{rr}
-0.6060 & -0.0232 \\
\hline-0.5728 & -0.0072
\end{array}\right],
$$

with $r_{\sigma}(\mathcal{L})=0.3884, \gamma^{*}=1.2165$, and $\left\|\mathcal{G}_{K}\right\|_{2}=1.0266$. 


\subsection{The $\mathcal{H}_{\infty}$ state-feedback control for hidden MJLS}

We introduce the following inequalities,

$$
\left.\begin{array}{cccc}
Q_{i} & \bullet \\
\Gamma_{i}(\Upsilon) Q_{i} & \Phi_{i}(Z)
\end{array}\right]>0,
$$

for all $i \in \mathbb{N}, l \in \mathbb{M}_{i}$. The set of variables of (4.17)-(4.18) for a given $\delta>0$ is represented by

$$
\xi_{\infty} \triangleq\left\{Q_{i}>0, Z_{i l}>0, i \in \mathbb{N}, l \in \mathbb{M}_{i}, G_{l}, Y_{l}, l \in \mathbb{M}\right\}
$$

and the set of all possible solutions of $(4.17)-(4.18)$ is $\Xi_{\infty} \triangleq$ $\left\{\xi_{\infty}\right.$ such that $(4.17)-(4.18)$ holds $\}$. We have the following lemma.

Lemma 4.2. Consider the following statements for a given $\delta>0$ :

(i) There exists $\xi_{\infty}$ such that $\xi_{\infty} \in \Xi_{\infty}$.

(ii) There exists a state-feedback controller $K$ such that $K \in \mathbb{K}$ and $\left\|\mathcal{G}_{K}\right\|_{\infty}<\delta$.

We have that $(i) \Longrightarrow($ ii $)$ by taking $K_{l}=Y_{l} G_{l}^{-1}$ for all $l \in \mathbb{M}$. Besides, if $\hat{\theta}=\theta$, then $(i i) \Longrightarrow(i)$.

Proof. If $\xi_{\infty} \in \Xi_{\infty}$, then applying the Schur complement to (4.17) yields

$$
Q_{i}^{-1}>\sum_{l \in \mathbb{M}_{i}} \alpha_{i l} Z_{i l}^{-1}
$$

Considering Lemma A.2 (see the Appendix A) in (4.18), setting $Y_{l}=K_{l} G_{l}$, and applying the congruence transformation $\operatorname{diag}\left(G_{l}^{-1}, I, I, I\right)$, and performing the Schur com- 
plement in the resulting inequality yields

$$
\left[\begin{array}{cccr}
Z_{i l}^{-1} & \bullet & \bullet & \bullet \\
0 & \delta^{2} I & \bullet & \bullet \\
A_{i l} & J_{i} & \left(\sum_{j \in \mathbb{N}} p_{i j} Q_{j}^{-1}\right)^{-1} & \bullet \\
C_{i l}^{(\infty)} & E_{i}^{(\infty)} & 0 & I
\end{array}\right]>0 .
$$

Since (3.27) and (4.19), and (3.27) and (4.20) are equivalent, we get that $K \in \mathbb{K}$ and $\left\|\mathcal{G}_{K}\right\|_{\infty}<\delta$.

Conversely, by applying a small perturbation $\epsilon_{i}>0$ in the optimal $\mathcal{H}_{\infty}$ design conditions of (GONÇALVES; FIORAVANTI; GEROMEL, 2012; OLIVEIRA, 2015) such that

$$
\left[\begin{array}{cccc}
G_{i}+G_{i}^{\prime}-\left(Q_{i}+I \epsilon_{i}\right) & \bullet & \bullet & \bullet \\
0 & \delta^{2} I & \bullet & \bullet \\
\Gamma_{i}(\mathbb{P})\left(A_{i} G_{i}+B_{i} Y_{i}\right) & \Gamma_{i}(\mathbb{P}) J_{i} & \Phi(Q) & \bullet \\
C_{i}^{(\infty)} G_{i}+D_{i}^{(\infty)} Y_{i} & E_{i}^{(\infty)} & 0 & I
\end{array}\right]>0
$$

still holds for all $i \in \mathbb{N}$, and defining $Q_{i}<Z_{i l}<Q_{i}+I \epsilon_{i}$, we get (4.17)-(4.18).

Remark 4.2: The set of conditions (4.17)-(4.18) is different from the one obtained in (TODOROV; FRAGOSO; COSTA, 2015). As we are going to see in Section 4.5, this formulation will be convenient to tackle the joint mixed $\mathcal{H}_{2} / \mathcal{H}_{\infty}$ problem, since it will allow us to obtain the same set of controller gains for both the $\mathcal{H}_{2}$ and $\mathcal{H}_{\infty}$ costs.

Bearing in mind the result of Lemma 4.2, we define

$$
\bar{\xi}_{\infty} \triangleq \xi_{\infty} \cup\left\{\delta_{a u x}\right\}
$$

for $\delta_{\text {aux }} \triangleq \delta^{2}$, and considering the set of all possible solutions of (4.10)-(4.11) given by $\bar{\Xi}_{\infty} \triangleq\left\{\bar{\xi}_{\infty}\right.$ such that $(4.17)-(4.18)$ holds $\}$, we write the $\mathcal{H}_{\infty}$ control problem:

$$
\inf _{\bar{\xi}_{\infty} \in \bar{\Xi}_{\infty}} \delta_{\text {aux }}
$$


Example 4.3 $\mathcal{H}_{\infty}$ state-feedback control: We use the system matrices of Examples 4.1 and 4.2 , and the controlled output matrices taken as follows

$$
C_{1}^{(\infty)}=\left[\begin{array}{ll}
1 & 0 \\
0 & 1 \\
0 & 0
\end{array}\right], C_{2}^{(\infty)}=\left[\begin{array}{ll}
0 & 1 \\
1 & 0 \\
0 & 0
\end{array}\right], D_{1}^{(\infty)}=D_{2}^{(\infty)}=\left[\begin{array}{l}
0 \\
0 \\
1
\end{array}\right],
$$

along with $E_{1}^{(\infty)}=E_{2}^{(\infty)}=0_{3 \times 3}$. We set $\rho_{1}=\rho_{2}=1$ (the mode-dependent case) and calculate the following MJLS $\mathcal{H}_{\infty}$ optimal state-feedback controller by (4.21)

$$
\left[\begin{array}{l}
K_{1} \\
K_{2}
\end{array}\right]=\left[\begin{array}{cc}
-0.6177 & -0.0635 \\
0 & 0
\end{array}\right],
$$

with $r_{\sigma}(\mathcal{L})=0.4041$ and the optimal cost given by $\left\|\mathcal{G}_{*}\right\|_{\infty}=\delta^{*}=2.3777$. On the other hand, we set $\rho_{1}=\rho_{2}=0.7$ and calculate the following state-feedback controller through (4.21),

$$
\left[\begin{array}{l}
K_{1} \\
K_{2}
\end{array}\right]=\left[\begin{array}{ll}
-0.4936 & -0.1518 \\
\hline-0.4776 & -0.1421
\end{array}\right],
$$

with $r_{\sigma}(\mathcal{L})=0.5380, \delta^{*}=6.5889$, and $\left\|\mathcal{G}_{K}\right\|_{\infty}=2.6734$.

\subsection{The mixed $\mathcal{H}_{2} / \mathcal{H}_{\infty}$ state-feedback control for hidden MJLS}

Lemmas 4.1 and 4.2 allow us to state the next theorem.

$$
\bar{\xi} \triangleq \xi_{2} \cup \xi_{\infty} \cup \xi_{\text {aux }}
$$

where $\xi_{\text {aux }}$ is the set that contains $\delta_{\text {aux }}, \gamma_{\text {aux }}$ or both upper bounds depending on the objective function $g(\gamma, \delta)$. We define $\bar{\Xi} \triangleq\{\bar{\xi}$ as in (4.24) such that the LMI (4.10) (4.11), (4.17) - (4.18) are jointly feasible\}.

Theorem 4.2. If there exists $\bar{\xi}$ such that $\bar{\xi} \in \bar{\Xi}$, then by setting $K_{l}=Y_{l} G_{l}^{-1}$ for all $l \in \mathbb{M}$, we get that $K \in \mathbb{K},\left\|\mathcal{G}_{K}\right\|_{2}<\gamma$, and $\left\|\mathcal{G}_{K}\right\|_{\infty}<\delta$.

Proof. The result is a consequence of Lemmas 4.1 and 4.2 and the use of the same variables $G_{l}$ and $Y_{l}$ for obtaining $K_{l}$. 
We write the following approximation for problem (4.7):

$$
\inf _{\bar{\xi} \in \bar{\Xi}} g(\gamma, \delta),
$$

where the function $g(\gamma, \delta)$ is set according to the problem of interest.

Remark 4.3: For obtaining a convex formulation for the $\mathcal{H}_{2}, \mathcal{H}_{\infty}$, and mixed $\mathcal{H}_{2} / \mathcal{H}_{\infty}$ control, we are obliged to perform the minimization on the upper bounds $\gamma$ and $\delta$, rather than on the actual $\mathcal{H}_{2}$ and $\mathcal{H}_{\infty}$ norms. We will usually refer to $\gamma$ and $\delta$ as guaranteed costs and the smallest values attained by (4.14), (4.21), and (4.25) as minimum guaranteed costs, $\gamma^{*}$ and $\delta^{*}$. A similar nomenclature is used, for instance, in (TODOROV; FRAGOSO, 2016; COSTA; FRAGOSO; TODOROV, 2015; TODOROV; FRAGOSO; COSTA, 2018). This jargon will be used, mutatis mutandis, throughout the work .

Remark 4.4: The property described in Corollary 3.2 allows us to simplify the result of Theorem 4.2 if $z(k)=z_{2}(k)=z_{\infty}(k)$. In this context, we set $C_{i}=C_{i}^{(2)}=C_{i}^{(\infty)}$, $D_{i}=D_{i}^{(2)}=D_{i}^{(\infty)}$, and $E_{i}=E_{i}^{(2)}=E_{i}^{(\infty)}$ for all $i \in \mathbb{N}$, along with $P_{i}=Q_{i}$ and $X_{i l}=Z_{i l}$ for all $i \in \mathbb{N}, l \in \mathbb{M}_{i}$. Then, even if we redefine $\bar{\xi}=\xi_{\infty} \cup\left\{W_{i}, i \in \mathbb{N}\right\} \cup \xi_{\text {aux }}$ and $\bar{\Xi}=\{\bar{\xi}$ such that the LMI (4.10) and (4.17) - (4.18) are jointly feasible $\}$, Theorem 4.2 would still hold.

Example 4.4 Mixed $\mathcal{H}_{2} / \mathcal{H}_{\infty}$ state-feedback control: We use the system matrices of Examples 4.1, 4.2, and 4.3 in order to obtain the best $\mathcal{H}_{2}$ guaranteed cost $g(\gamma, \delta)=\gamma^{2}$ for a given $\delta>0$ (Problem 1). By initially considering $\rho_{1}=\rho_{2}=1$ (the mode-dependent case) and setting $\delta=3$, and calculate the following MJLS $\mathcal{H}_{\infty}$ optimal state-feedback controller by (4.25)

$$
\left[\begin{array}{l}
K_{1} \\
K_{2}
\end{array}\right]=\left[\begin{array}{cc}
-0.6913 & -0.0143 \\
0 & 0
\end{array}\right],
$$

with $r_{\sigma}(\mathcal{L})=0.3881$, along with $\gamma^{*}=1.0839,\left\|\mathcal{G}_{K}\right\|_{2}=0.9979,\left\|\mathcal{G}_{K}\right\|_{\infty}=2.4255$. On the other hand, we set $\rho_{1}=\rho_{2}=0.7$ and $\delta=7$, and calculate the following state-feedback controller through (4.25),

$$
\left[\begin{array}{l}
K_{1} \\
K_{2}
\end{array}\right]=\left[\begin{array}{ll}
-0.5505 & -0.1277 \\
\hline-0.5336 & -0.1167
\end{array}\right],
$$

with $r_{\sigma}(\mathcal{L})=0.4744, \gamma^{*}=1.5921,\left\|\mathcal{G}_{K}\right\|_{2}=1.0955$, and $\left\|\mathcal{G}_{K}\right\|_{\infty}=2.4880$. 


\subsection{State-feedback control for the cluster case and robust control}

We focus our efforts on the equivalence between the detector approach under Assumption 3.1 and the cluster case of (VAL; GEROMEL; GONÇALVES, 2002). For that, consider the following inequality set taken from (GONÇALVES, 2014; OLIVEIRA, 2015),

$$
\begin{gathered}
\sum_{i \in \mathbb{N}} \mu_{i} \operatorname{Tr}\left(W_{i}\right)<\gamma^{2}, \quad\left[\begin{array}{ccc}
W_{i} & \bullet & \bullet \\
\Gamma_{i}(\mathbb{P}) J_{i} & \Phi(P) & \bullet \\
E_{i}^{(2)} & 0 & I
\end{array}\right]>0, \\
\\
{\left[\begin{array}{ccc}
G^{s}+G^{s \prime}-P_{i} & \bullet & \bullet \\
\Gamma_{i}(\mathbb{P})\left(A_{i} G^{s}+B_{i} Y^{s}\right) & \Phi(P) & \bullet \\
C_{i}^{(2)} G^{s}+D_{i}^{(2)} Y^{s} & 0 & I
\end{array}\right]>0,}
\end{gathered}
$$

as well as the following conditions taken from (GONÇALVES et al., 2011; GONÇALVES; FIORAVANTI; GEROMEL, 2012; OLIVEIRA, 2015)

$$
\left[\begin{array}{cccc}
G^{s}+G^{s \prime}-Q_{i} & \bullet & \bullet & \bullet \\
0 & \delta^{2} I & \bullet & \bullet \\
\Gamma_{i}(\mathbb{P})\left(A_{i} G^{s}+B_{i} Y^{s}\right) & \Gamma_{i}(\mathbb{P}) J_{i} & \Phi(Q) & \bullet \\
C_{i}^{(\infty)} G^{s}+D_{i}^{(\infty)} Y^{s} & E_{i}^{(\infty)} & 0 & I
\end{array}\right]>0,
$$

for all $s \in\{1, \ldots, \kappa\}, i \in \mathbb{N}^{s}$. We have the following proposition.

Proposition 4.1: Under Assumption 3.1, we have that

- Conditions (4.10)-(4.11) and (4.28)-(4.29) are equivalent;

- Conditions (4.17)-(4.18) and (4.30) are equivalent .

Proof. Considering that (4.10)-(4.11) hold, we multiply the second inequality of (4.11) by $\alpha_{l}^{s}$, sum it up for $l \in \mathbb{M}^{s}$, and define

$$
G^{s}=\sum_{l \in \mathbb{M}^{s}} \alpha_{l}^{s} G_{l}, Y^{s}=\sum_{l \in \mathbb{M}^{s}} \alpha_{l}^{s} Y_{l}, X_{i}^{s}=\sum_{l \in \mathbb{M}^{s}} \alpha_{l}^{s} X_{i l}
$$


in order to get that

$$
\left[\begin{array}{ccc}
G^{s}+G^{s^{\prime}}-X_{i}^{s} & \bullet & \bullet \\
\Gamma_{i}(\mathbb{P})\left(A_{i} G^{s}+B_{i} Y^{s}\right) & \Gamma_{i}(\mathbb{P}) J_{i} & \Phi(P) \\
C_{i}^{(2)} G^{s}+D_{i}^{(2)} Y^{s} & 0 & I
\end{array}\right]>0,
$$

for all $s \in\{1, \ldots, \kappa\}, i \in \mathbb{N}^{s}$. Applying the congruence transformation $\operatorname{diag}\left(P_{i}^{-1}, I\right)$ and the Schur complement to the first inequality of (4.11), and noting, by Lemma A.4 (see the Appendix A), that $\sum_{l \in \mathbb{M}^{s}} \alpha_{l}^{s} X_{i l} \geq\left(\sum_{l \in \mathbb{M}^{s}} \alpha_{l}^{s} X_{i l}^{-1}\right)^{-1}$, we get by the first inequality of (4.11) that

$$
\left[\begin{array}{cc}
P_{i}^{-1} & \bullet \\
I & \sum_{l \in \mathbb{M}^{s}} \alpha_{l}^{s} X_{i l}
\end{array}\right]>0,
$$

for all $i \in \mathbb{N}$. Applying the Schur complement to the inequality above yields $X_{i}{ }^{s}>P_{i}$ and consequently $G^{s}+G^{s \prime}-P_{i}>G^{s}+G^{s \prime}-X_{i}^{s}$, that allows us to rewrite (4.31) as (4.29).

On the other hand, define $X_{i l}$ such that $P_{i}<X_{i l}<P_{i}+I \epsilon_{i}$ for some suitable $\epsilon_{i}>0$ such that

$$
\left[\begin{array}{ccc}
G^{s}+G^{s \prime}-\left(P_{i}+I \epsilon_{i}\right) & \bullet & \bullet \\
\Gamma_{i}(\mathbb{P})\left(A_{i} G^{s}+B_{i} Y^{s}\right) & \Phi(P) & \bullet \\
C_{i}^{(2)} G^{s}+D_{i}^{(2)} Y^{s} & 0 & I
\end{array}\right]>0
$$

still holds. It follows from the definition of $X_{i l}$ that $P_{i}^{-1}>\sum_{l \in \mathbb{M}_{i}} \alpha_{l}^{s} X_{i l}^{-1}$, a condition that retrieves the first inequality of (4.11). Besides, by setting $G^{s}=G_{l}$ and $Y^{s}=Y_{l}$, and considering that the definition of $X_{i l}$ implies that $G_{l}+G_{l}^{\prime}-X_{i l}>G^{s}+G^{s \prime}-\left(P_{i}+I \epsilon_{i}\right)$ allows us to rewrite (4.32) as the second inequality of (4.11). The similar reasoning can be applied to (4.30) to (4.17)-(4.18), and the claim follows.

We now study the case in which the transition probability matrix and the detection probabilities are not exactly known, but are described by $\mathbb{P} \in \mathbb{D}_{\mathbb{P}}$ and $\Upsilon \in \mathbb{D}_{\Upsilon}$, for $\mathbb{D}_{\mathbb{P}}$ and $\mathbb{D}_{\Upsilon}$ given respectivelly in (3.3) and (3.4). In this case, we define the set $\mathbb{K}_{r} \triangleq\{K$ : (4.3) for $\mathbb{P} \in \mathbb{D}_{\mathbb{P}}$ and $\Upsilon \in \mathbb{D}_{\Upsilon}$ is SS , set

$$
\begin{aligned}
& \Gamma_{i}(\mathbb{P})=\Gamma_{i}\left(\mathbb{P}_{k}\right), \\
& \Gamma_{i}(\Upsilon)=\Gamma_{i}\left(\Upsilon_{t}\right),
\end{aligned}
$$


in (4.10)-(4.11) and (4.17)-(4.18) and define $\tilde{\Xi} \triangleq\{\bar{\xi}$ such that $(4.10)-(4.11),(4.17)-$ (4.18), and (4.33) jointly hold\}.

Corollary 4.1. If $\bar{\xi} \in \tilde{\Xi}$, then by setting $K_{l}=Y_{l} G_{l}^{-1}$ for all $l \in \mathbb{M}$, we get that $K \in \mathbb{K}_{r}$, $\left\|\mathcal{G}_{K}\right\|_{2}<\gamma$, and $\left\|\mathcal{G}_{K}\right\|_{\infty}<\delta$.

Proof. We can rewrite (4.11) subject to (4.33) as follows

$$
P_{i}^{-1}>\sum_{l \in \mathbb{M}_{i}} \alpha_{i l}^{(t)} X_{i l}^{-1} \geq \sum_{l \in \mathbb{M}_{i}} \alpha_{i l}^{(t)}\left(A_{i l}^{\prime}\left(\sum_{j \in \mathbb{N}} p_{i j}^{(h)} P_{j}^{-1}\right) A_{i l}+C_{i l}^{(2)^{\prime}} C_{i l}^{(2)}\right) .
$$

Multiplying the result by $a_{h}$ and summing the result up for all $h \in\{1, \ldots, \eta\}$, as well as by $b_{t}$ and summing it up for all $t \in\{1, \ldots, \tau\}$ yields (3.16). The same reasoning can applied to (4.10), (4.17) and (4.18) and the claim follows.

Example 4.5 Mixed $\mathcal{H}_{2} / \mathcal{H}_{\infty}$ state-feedback control for the cluster case: In this example, we set $\rho_{1}=\rho_{2}=0.5$ and $\rho_{3}=1$ in (4.9). Note that this choice of $\rho_{i}$ in (4.9) respects Assumption 3.1. By taking $\delta=8$ and solving (4.25) for $g(\gamma, \delta)=\gamma^{2}$, we get the following controller

$$
K_{i}=\left[\begin{array}{ll}
-0.5416 & -0.1276
\end{array}\right]
$$

for all $i \in \mathbb{N}$, with $r_{\sigma}(\mathcal{L})=0.4797, \gamma^{*}=1.6103,\left\|\mathcal{G}_{K}\right\|_{2}=1.1000$, and $\left\|\mathcal{G}_{K}\right\|_{\infty}=2.4987$. Note the "clusterization effect" $\mathbb{N}^{1}=\{1,2\}$ induced by the choice of conditional probabilities.

\subsection{Final remarks}

In this chapter, we studied the state-feedback control for hidden MJLS, assuming that the controller has access only to the observable variable $\hat{\theta}(k)$. We provided design conditions in terms of LMls for stabilizing, $\mathcal{H}_{2}, \mathcal{H}_{\infty}$, and mixed $\mathcal{H}_{2} / \mathcal{H}_{\infty}$ state-feedback controllers and studied also the cluster case through the lens of the detector approach. Numerical examples were given in order to illustrate the results. 


\section{FILTERING FOR HIDDEN MJLS}

In this chapter we study the $\mathcal{H}_{2}, \mathcal{H}_{\infty}$, and mixed $\mathcal{H}_{2} / \mathcal{H}_{\infty}$ filtering design for hidden MJLS. We present design conditions in the LMI formulation for the $\mathcal{H}_{2}, \mathcal{H}_{\infty}$, and mixed $\mathcal{H}_{2} / \mathcal{H}_{\infty}$ filtering in order to get full order filters depending only on $\hat{\theta}(k)$. The results concerning $\mathcal{H}_{2}$ filters can be found in (OLIVEIRA; COSTA, 2017a) and the $\mathcal{H}_{\infty}$ filters, in (OLIVEIRA; COSTA, 2016b; OLIVEIRA; COSTA, 2017b). The mixed case in a different formulation from the one given in this thesis can be found in (OLIVEIRA; COSTA, 2018b). We also investigate the Bernoulli case and the relationship of our results to the cluster case in (GONÇALVES; FIORAVANTI; GEROMEL, 2010).

This chapter is organized as follows. In Section 5.1 we present the filtering problems that we are concerned, along with the filter structure. Sections 5.2, 5.3, and 5.4 deal with the $\mathcal{H}_{2}, \mathcal{H}_{\infty}$, and mixed $\mathcal{H}_{2} / \mathcal{H}_{\infty}$ filtering for hidden MJLS, respectively. The cluster case and the robust filtering is tackled in Section 5.5 and the Bernoulli formulation, in Section 5.6. Our final remarks are stated in Section 5.7.

\subsection{Problem formulation}

On a probabilistic space $\left(\Omega, \mathfrak{F}\right.$, Prob) with filtration $\left\{\mathfrak{F}_{k}\right\}$, we consider the following Markov jump linear system (MJLS)

$$
\mathcal{G}:\left\{\begin{aligned}
x(k+1) & =A_{\theta(k)} x(k)+J_{\theta(k)} w(k) \\
y(k) & =L_{\theta(k)} x(k)+H_{\theta(k)} w(k) \\
z_{2}(k) & =C_{\theta(k)}^{(2)} x(k)+E_{\theta(k)}^{(2)} w(k) \\
z_{\infty}(k) & =C_{\theta(k)}^{(\infty)} x(k)+E_{\theta(k)}^{(\infty)} w(k) \\
x(0) & =x_{0},
\end{aligned}\right.
$$

where $x(k) \in \mathbb{R}^{n}$ is the state variable, $w(k) \in \mathbb{R}^{r}$ is the input variable, $y(k) \in \mathbb{R}^{p}$ is the measured output, $z_{2}(k) \in \mathbb{R}^{q_{2}}$ and $z_{\infty}(k) \in \mathbb{R}^{q_{\infty}}$ are the outputs to be estimated related to the $\mathcal{H}_{2}$ and $\mathcal{H}_{\infty}$ performance criteria. In general, $x_{0}=0$, unless for the stochastic $\mathcal{H}_{2}$ filtering where it can be taken as a second order random vector, with $\mathbf{E}\left(x_{0}\right)=0$ and $\theta_{0} \sim \mu$, for $\mu_{i}>0, i \in \mathbb{N}$, as usual. 
We want to synthesize filters with the following structure:

$$
\mathcal{F}:\left\{\begin{aligned}
x_{f}(k+1) & =A_{f \hat{\theta}(k)} x_{f}(k)+B_{f \hat{\theta}(k)} y(k) \\
z_{f}(k) & =C_{f \hat{\theta}(k)} x_{f}(k)+D_{f \hat{\theta}(k)} y(k) \\
x_{f}(0) & =0
\end{aligned}\right.
$$

where $x_{f}(k) \in \mathbb{R}^{n}$ and $z_{f}(k) \in \mathbb{R}^{q}$, that is whose matrices depend only on the detector output $\hat{\theta}(k)$. We define the error signals such that

$$
\begin{gathered}
e_{2}(k) \triangleq z_{2}(k)-z_{f}(k), \\
e_{\infty}(k) \triangleq z_{\infty}(k)-z_{f}(k) .
\end{gathered}
$$

Note that the definition of $e_{2}$ and $e_{\infty}$ above imposes that $q_{2}=q_{\infty}=q$. Connecting (5.2) to the output of (5.1) yields to the following extended system:

$$
\mathcal{G}_{f}:\left\{\begin{aligned}
\tilde{x}(k+1) & =A_{\theta(k) \hat{\theta}(k)} \tilde{x}(k)+J_{\theta(k) \hat{\theta}(k)} w(k) \\
e_{2}(k) & =C_{\theta(k) \hat{\theta}(k)}^{(2)} \tilde{x}(k)+E_{\theta(k) \hat{\theta}(k)}^{(2)} w(k) \\
e_{\infty}(k) & =C_{\theta(k) \hat{\theta}(k)}^{(\infty)} \tilde{x}(k)+E_{\theta(k) \hat{\theta}(k)}^{(\infty)} w(k),
\end{aligned}\right.
$$

where $\tilde{x}(k) \in \mathbb{R}^{2 n}$ is defined as $\tilde{x}(k)^{\prime}=\left[x(k)^{\prime} \quad x_{f}(k)^{\prime}\right]$, along with

$$
\left[\begin{array}{c|c}
A_{i l} & J_{i l} \\
\hline C_{i l}^{(2)} & E_{i l}^{(2)} \\
\hline C_{i l}^{(\infty)} & E_{i l}^{(\infty)}
\end{array}\right]=\left[\begin{array}{cc|c}
A_{i} & 0 & J_{i} \\
B_{f l} L_{i} & A_{f l} & B_{f l} H_{i} \\
\hline C_{i}^{(2)}-D_{f l} L_{i} & -C_{f l} & E_{i}^{(2)}-D_{f l} H_{i} \\
\hline C_{i}^{(\infty)}-D_{f l} L_{i} & -C_{f l} & E_{i}^{(\infty)}-D_{f l} H_{i}
\end{array}\right],
$$

for $i \in \mathbb{N}, l \in \mathbb{M}_{i}$. The set of admissible filters is represented by

$\mathbb{F} \triangleq\{\mathcal{F}$ as in (5.2) such that (5.3) is stochastically stable $\}$.

We need the following assumption.

Assumption 5.1: System (5.1) is stochastically stable.

Remark 5.1: For the mode-dependent case discussed in Chapter 3, Section 3.2, that occurs whenever $\hat{\theta}=\theta$, we have that Assumption 5.1 could be suppressed since the filter could be rewritten in a Markov observer form and, in this case, the Markov dynamic equation for the errors could be stochastically stable even if the original system is not (see, for instance, (COSTA; FRAGOSO; MARQUES, 2005)). However, for the general 
case, we adopt a similar approach as the one used in the cluster observation, see for instance (GONÇALVES; FIORAVANTI; GEROMEL, 2010), in which Assumption 5.1 is required.

The problem is presented below

$$
\inf _{\mathcal{F} \in \mathbb{F}}\left\{g(\gamma, \delta) ; \text { such that }\left\|G_{f}\right\|_{2}<\gamma \text { and }\left\|G_{f}\right\|_{\infty}<\delta\right\},
$$

where $\left\|G_{f}\right\|_{2}$ is related to the error signal $e_{2}$ (see Definition 3.2) and $\left\|G_{f}\right\|_{\infty}$, to $e_{\infty}$ (see Definition 3.3). The objective function $g(\gamma, \delta)$ can be chosen as follows:

Filtering Project 1 (The mixed $\mathcal{H}_{2} / \mathcal{H}_{\infty}$ filtering problem). Find $\mathcal{F} \in \mathbb{F}$ such that a suitable upper bound for the $\mathcal{H}_{2}$ norm of (5.3) is obtained, subject to a given upper bound $\delta>0$ on the $\mathcal{H}_{\infty}$ norm:

$$
g(\gamma, \delta)=\gamma^{2} .
$$

Filtering Project 2 (The mixed "inverse" $\mathcal{H}_{2} / \mathcal{H}_{\infty}$ filtering problem). Find $\mathcal{F} \in \mathbb{F}$ such that a suitable upper bound for the $\mathcal{H}_{\infty}$ norm of (5.3) is obtained, subject to a given upper bound $\gamma>0$ on the $\mathcal{H}_{2}$ norm:

$$
g(\gamma, \delta)=\delta^{2} .
$$

Filtering Project 3 (A weighted combination of the $\mathcal{H}_{2}$ and $\mathcal{H}_{\infty}$ costs for the filtering problem). Find $\mathcal{F} \in \mathbb{F}$ such that weighted combinations of suitable upper bounds for both $\mathcal{H}_{2}$ and $\mathcal{H}_{\infty}$ norm of (5.3) are obtained. Thus, for given $\beta^{(2)} \geq 0$ and $\beta^{(\infty)} \geq 0$, we set

$$
g(\gamma, \delta)=\gamma^{2} \beta^{(2)}+\delta^{2} \beta^{(\infty)}
$$

Similarly to the first approach of Chapter 4 , a first method is to minimize the objective function $g(\gamma, \delta)$ subject to (3.19)-(3.20) and (3.25) with system matrices given in (5.4):

$$
\inf _{\mathcal{F} \in \mathbb{E}, P, W, Q, \gamma, \delta}\{g(\gamma, \delta): \text { such that (3.19) - (3.20) and (3.25) holds }\} .
$$

This problem is non-linear and hard to solve in $\mathcal{F}, P, W$ and $Q$. However, due to the form of (3.19)-(3.20) and (3.25), the same linearization procedures devised in (FIORA- 
VANTI; GONÇALVES; GEROMEL, 2008), (GONÇALVES; FIORAVANTI; GEROMEL, 2009), and (GONÇALVES; FIORAVANTI; GEROMEL, 2010) can be used for both the $\mathcal{H}_{2}$ and $\mathcal{H}_{\infty}$ filtering. Specially, the work (GONÇALVES; FIORAVANTI; GEROMEL, 2010) introduced slack variables that is an essential tool for our results.

\section{2 $\quad \mathcal{H}_{2}$ filtering for hidden MJLS}

Consider the following inequality set, for all $i \in \mathbb{N}, l \in \mathbb{M}_{i}$, and a given $\gamma>0$ :

$$
\begin{aligned}
& \sum_{i \in \mathbb{N}} \sum_{l \in \mathbb{M}_{i}} \mu_{i} \alpha_{i l} \operatorname{Tr}\left(W_{i l}\right)<\gamma^{2}, \\
& {\left[\begin{array}{cccc}
W_{i l} & \bullet & \bullet & \bullet \\
\mathcal{E}_{i}(Y) J_{i} & \mathcal{E}_{i}(Y) & \bullet & \bullet \\
G_{l} J_{i}+F_{l} H_{i} & 0 & \operatorname{Her}\left(G_{l}\right)+\mathcal{E}_{i}(Y)-\mathcal{E}_{i}(X) & \bullet \\
E_{i}^{(2)}+K_{l} H_{i} & 0 & 0 & I
\end{array}\right]>0,} \\
& {\left[\begin{array}{cc}
Y_{i} & \bullet \\
Y_{i} & X_{i}
\end{array}\right]>\sum_{l \in \mathbb{M}_{i}} \alpha_{i l}\left[\begin{array}{cccc}
M_{i l}^{(2,1,1)} & \bullet \\
M_{i l}^{(2,2,1)} & M_{i l}^{(2,2,2)}
\end{array}\right],} \\
& {\left[\begin{array}{ccccc}
M_{i l}^{(2,1,1)} & \bullet & \bullet & \bullet \\
M_{i l}^{(2,2,1)} & M_{i l}^{(2,2,2)} & \bullet & \bullet & \bullet \\
\mathcal{E}_{i}(Y) A_{i} & \mathcal{E}_{i}(Y) A_{i} & \mathcal{E}_{i}(Y) & \bullet & \bullet \\
G_{l} A_{i}+F_{l} L_{i}+R_{l} & G_{l} A_{i}+F_{l} L_{i} & 0 & \operatorname{Her}\left(G_{l}\right)+\mathcal{E}_{i}(Y)-\mathcal{E}_{i}(X) & \bullet \\
C_{i}^{(2)}+K_{l} L_{i}+O_{l} & C_{i}^{(2)}+K_{l} L_{i} & 0 & 0 & \bullet
\end{array}\right]>0,}
\end{aligned}
$$

We define the following sets,

$$
\begin{aligned}
\xi_{(2, a)} & \triangleq\left\{Y_{i}, X_{i} \text { for all } i \in \mathbb{N}\right\}, \\
\xi_{(f)} & \triangleq\left\{G_{l}, R_{l}, F_{l}, K_{l}, O_{l} \text { for all } l \in \mathbb{M}\right\}, \\
\xi_{(2, b)} & \triangleq\left\{M_{i l}^{(2,1,1)}, M_{i l}^{(2,2,1)}, M_{i l}^{(2,2,2)} \text { for all } i \in \mathbb{N}, l \in \mathbb{M}_{i}\right\},
\end{aligned}
$$

such that $\xi_{2} \triangleq \xi_{(2, a)} \cup \xi_{(f)} \cup \xi_{(2, b)}$, and so the set of all possible solutions of (5.7)-(5.10) for a given $\gamma$ is given by $\Xi_{2} \triangleq\left\{\xi_{2}\right.$ such that (5.7) $-(5.10)$ holds $\}$. We have the following lemma.

Lemma 5.1. Consider the following statements for a given $\gamma>0$ :

(i) There exists $\xi_{2}$ such that $\xi_{2} \in \Xi_{2}$. 
(ii) There exists $\mathcal{F} \in \mathbb{F}$ with $\left\|\mathcal{G}_{f}\right\|_{2}<\gamma$.

We have that $(i) \Longrightarrow$ (ii) by setting $A_{f l}=-G_{l}^{-1} R_{l}, B_{f l}=-G_{l}^{-1} F_{l}, C_{f l}=-O_{l}$ and $D_{f l}=-K_{l}$ for all $l \in \mathbb{M}$. If $\hat{\theta}=\theta$, then $(i i) \Longrightarrow(i)$.

Proof. We follow the similar lines as in the proofs given in (GONÇALVES; FIORAVANTI; GEROMEL, 2010). Considering that $\xi_{2} \in \Xi_{2}$ and setting $R_{l}=-G_{l} A_{f l}$, $F_{l}=-G_{l} B_{f l}, O_{l}=-C_{f l}$, and $K_{l}=-D_{f l}$, and recalling Lemma A.2 allows us to infer that

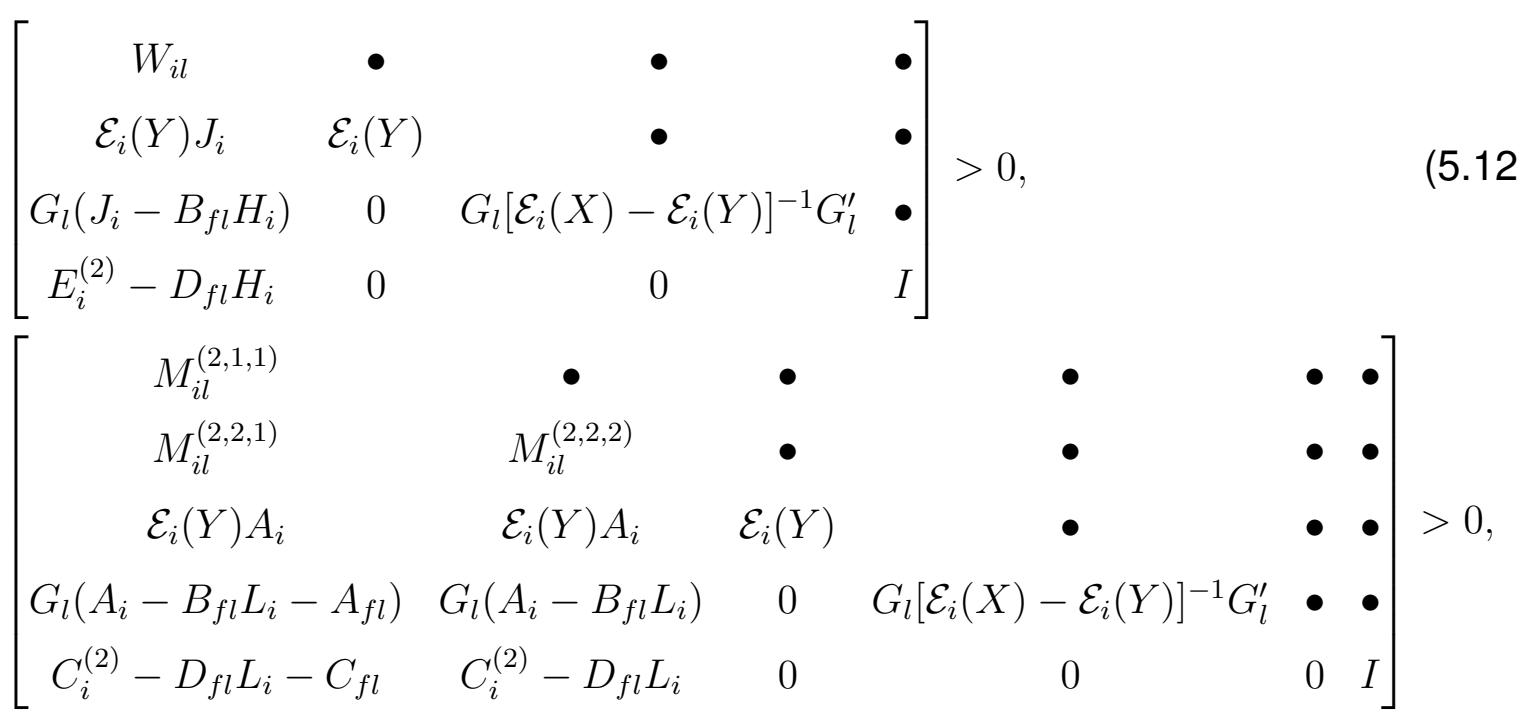

also hold for all $i \in \mathbb{N}, l \in \mathbb{M}_{i}$. As in (GONÇALVES; FIORAVANTI; GEROMEL, 2010), we define the matrix

$$
\mathcal{D}_{i l}^{(2)} \triangleq\left[\begin{array}{cc}
I & I \\
0 & G_{l}{ }^{-T}\left[\mathcal{E}_{i}(X)-\mathcal{E}_{i}(Y)\right]
\end{array}\right]
$$

and apply the congruence transformations $\operatorname{diag}\left(I_{r}, \mathcal{D}_{i l}^{(2)}, I_{q_{2}}\right)$ and $\operatorname{diag}\left(I_{2 n}, \mathcal{D}_{i l}^{(2)}, I_{q_{2}}\right)$ to (5.12) and (5.13) in order to obtain that

$$
\left[\begin{array}{cccc}
W_{i l} & \bullet & \bullet & \bullet \\
\mathcal{E}_{i}(Y) J_{i} & \mathcal{E}_{i}(Y) & \bullet & \bullet \\
\mathcal{E}_{i}(X) J_{i}+\mathcal{E}_{i}(Y-X) B_{f l} H_{i} & \mathcal{E}_{i}(Y) & \mathcal{E}_{i}(X) & \bullet \\
E_{i}^{(2)}-D_{f l} H_{i} & 0 & 0 & I
\end{array}\right]>0,
$$




$$
\left[\begin{array}{cccccc}
M_{i l}^{(2,1,1)} & \bullet & \bullet & \bullet & \bullet & \bullet \\
M_{i l}^{(2,2,1)} & M_{i l}^{(2,2,2)} & \bullet & \bullet & \bullet & \bullet \\
\mathcal{E}_{i}(Y) A_{i} & \mathcal{E}_{i}(Y) A_{i} & \mathcal{E}_{i}(Y) & \bullet & \bullet & \bullet \\
\mathcal{X}_{i l}^{(1)}+\mathcal{E}_{i}(Y-X) A_{f l} & \mathcal{X}_{i l}^{(1)} & \mathcal{E}_{i}(Y) & \mathcal{E}_{i}(X) & \bullet & \bullet \\
C_{i}^{(2)}-D_{f l} L_{i}-C_{f l} & C_{i}^{(2)}-D_{f l} L_{i} & 0 & 0 & 0 & I
\end{array}\right]>0,
$$

where $\mathcal{X}_{i l}^{(1)} \triangleq \mathcal{E}_{i}(X) A_{i}+\mathcal{E}_{i}(Y-X) B_{f l} L_{i}$. By restricting the structure of $P_{i}$ in (3.19)-(3.20) as shown in (A.1) and setting $\hat{U}_{i}=-\hat{X}_{i}$, we have that $\hat{V}_{i} Y_{i}=I$ and $Y_{i}=\hat{U}_{i}+X_{i}$, and consequently $\hat{P}_{1 i}^{-1}=\mathcal{E}_{i}(Y)$. This allows us to rewrite (5.14) and (5.15) in the following form:

$$
\left[\begin{array}{ccc}
W_{i l} & \bullet & \bullet \\
\mathcal{H}_{i}^{(2)^{\prime}} J_{i l} & \mathcal{H}_{i}^{(2)^{\prime}} \mathcal{E}_{i}(P)^{-1} \mathcal{H}_{i}^{(2)} & \bullet \\
E_{i l}^{(2)} & 0 & I
\end{array}\right]>0, \quad\left[\begin{array}{ccc}
\mathcal{T}_{i}^{(2)^{\prime}} M_{i l}^{(2)} \mathcal{T}_{i}^{(2)} & \bullet & \bullet \\
\mathcal{H}_{i}^{(2)^{\prime}} A_{i l} \mathcal{T}_{i}^{(2)} & \mathcal{H}_{i}^{(2)^{\prime}} \mathcal{E}_{i}(P)^{-1} \mathcal{H}_{i}^{(2)} & \bullet \\
C_{i l}^{(2)} \mathcal{T}_{i}^{(2)} & 0 & I
\end{array}\right]>0
$$

as well as (5.9) as $\mathcal{T}_{i}^{(2)^{\prime}} P_{i} \mathcal{T}_{i}^{(2)}>\sum_{l \in \mathbb{M}_{i}} \alpha_{i l} \mathcal{T}_{i}^{(2)^{\prime}} M_{i l}^{(2)} \mathcal{T}_{i}^{(2)}$, for system matrices shown in (5.4) and $\mathcal{T}_{i}^{(2)}$ and $\mathcal{H}_{i}^{(2)}$ as in (A.3) and (A.5), respectively. Applying the congruence transformations $\operatorname{diag}\left(I_{r}, \mathcal{H}_{i}^{(2)^{-1}}, I_{q_{2}}\right), \operatorname{diag}\left(\mathcal{T}_{i}^{(2)^{-1}}, \mathcal{H}_{i}^{(2)^{-1}}, I_{q_{2}}\right)$, and $\mathcal{T}_{i}^{(2)^{-1}}$ to the previous inequalities, and considering (5.7), we get (3.19)-(3.20) with system matrices given in (5.4).

Conversely, if $\hat{\theta}=\theta$, we get that $\alpha_{i i}=1$ for all $i \in \mathbb{N}$. By considering that the Equation (14) in (GONÇALVES; FIORAVANTI; GEROMEL, 2010) still holds by applying a small perturbation $\epsilon_{i}>0$ in the first two blocks of the main diagonal and defining $M_{i l}^{(2,1,1)}, M_{i l}^{(2,2,1)}$, and $M_{i l}^{(2,2,2)}$ such that

$$
\left[\begin{array}{cc}
Y_{i}-\epsilon_{i} I & \bullet \\
Y_{i} & X_{i}-\epsilon_{i} I
\end{array}\right]<\left[\begin{array}{cc}
M_{i l}^{(2,1,1)} & \bullet \\
M_{i l}^{(2,2,1)} & M_{i l}^{(2,2,2)}
\end{array}\right]<\left[\begin{array}{cc}
Y_{i} & \bullet \\
Y_{i} & X_{i}
\end{array}\right]
$$

holds, we get (5.9)-(5.10).

By defining the following set $\bar{\xi}_{2} \triangleq \xi_{2} \cup\left\{\gamma_{\text {aux }}\right\}$, where $\gamma_{\text {aux }} \triangleq \gamma^{2}$, and considering the set of all possible solutions of (5.7)-(5.10) given by $\bar{\Xi}_{2} \triangleq\left\{\bar{\xi}_{2}\right.$ such that (5.7) $-(5.10)$ holds $\}$, we write the $\mathcal{H}_{2}$ filtering problem as follows,

$$
\inf _{\bar{\xi}_{2} \in \bar{\Xi}_{2}} \gamma_{\text {aux }}
$$


Example 5.1 $\mathcal{H}_{2}$ filtering for hidden MJLS: We consider a modified version of the system in (SOUZA, 2003). System matrices are as follows:

$$
A_{1}=\left[\begin{array}{rr}
0 & -0.5 \\
1.0 & 1.0
\end{array}\right], A_{2}=\left[\begin{array}{rr}
0 & -0.33 \\
1.00 & 1.40
\end{array}\right], J_{i}=\left[\begin{array}{cc}
0.5 & 0 \\
0 & 0
\end{array}\right], L_{i}=\left[\begin{array}{l}
1 \\
0
\end{array}\right]^{\prime}, H_{i}=\left[\begin{array}{l}
0 \\
1
\end{array}\right]^{\prime}
$$

for $i \in \mathbb{N}$. For the $\mathcal{H}_{2}$ filtering, we set

$$
C_{i}^{(2)}=\left[\begin{array}{ll}
1 & 0 \\
0 & 1
\end{array}\right], \quad E_{i}^{(2)}=0_{2 \times 2},
$$

for $i \in \mathbb{N}$, that is, we want the best estimate of $e_{1}(k)$ and $e_{2}(k)$ in the presence of white noise. The transition probabilities are taken as

$$
\mathbb{P}=\left[\begin{array}{ll}
0.8 & 0.2 \\
0.4 & 0.6
\end{array}\right]
$$

and $\mu$ is taken as the stationary distribution. We consider the following structure for the conditional probabilities

$$
\Upsilon=\left[\begin{array}{cc}
\rho_{1} & 1-\rho_{1} \\
1-\rho_{2} & \rho_{2}
\end{array}\right]
$$

and initially set $\rho_{1}=\rho_{2}=1$. By solving (5.16), we get the following filter matrices

$$
\left[\begin{array}{l|l}
A_{f 1} & B_{f 1} \\
\hline C_{f 1} & D_{f 1}
\end{array}\right]=\left[\begin{array}{rr|r}
-0.1364 & -0.5000 & 0.1364 \\
0.9487 & 1.0000 & 0.0513 \\
\hline 0.6759 & -0.0000 & 0.3241 \\
0.2728 & 1.0000 & -0.2728
\end{array}\right]
$$

and

$$
\left[\begin{array}{l|l}
A_{f 2} & B_{f 2} \\
\hline C_{f 2} & D_{f 2}
\end{array}\right]=\left[\begin{array}{rr|r}
-0.1154 & -0.3300 & 0.1154 \\
1.1791 & 1.4000 & -0.1791 \\
\hline 0.6895 & -0.0000 & 0.3105 \\
0.3497 & 1.0000 & -0.3497
\end{array}\right],
$$

with $\gamma^{*}=\left\|\mathcal{G}_{*}\right\|_{2}=1.2224$. We now set $\rho_{1}=\rho_{2}=0.7$ in (5.17) and calculate the following 
filter matrices

$$
\left[\begin{array}{l|l}
A_{f 1} & B_{f 1} \\
\hline C_{f 1} & D_{f 1}
\end{array}\right]=\left[\begin{array}{rr|r}
-0.3933 & -0.5708 & 0.1498 \\
1.0658 & 0.9999 & -0.0075 \\
\hline 0.7191 & -0.0801 & 0.3353 \\
0.5830 & 1.1099 & -0.3169
\end{array}\right]
$$

and

$$
\left[\begin{array}{c|c}
A_{f 2} & B_{f 2} \\
\hline C_{f 2} & D_{f 2}
\end{array}\right]=\left[\begin{array}{rr|r}
-0.2114 & -0.4476 & 0.1429 \\
0.5339 & 0.7748 & -0.1049 \\
\hline 0.7764 & 0.3963 & 0.3212 \\
0.0976 & 0.6319 & -0.3453
\end{array}\right]
$$

with $\gamma^{*}=1.2868$ and $\left\|\mathcal{G}_{f}\right\|_{2}=1.2842$.

\section{3 $\mathcal{H}_{\infty}$ filtering for hidden MJLS}

We introduce the following inequality set, for all $i \in \mathbb{N}, l \in \mathbb{M}_{i}$, and a given $\delta>0$ :

$$
\begin{aligned}
& {\left[\begin{array}{ccc}
Z_{i} & \bullet & \bullet \\
Z_{i} & T_{i} & \bullet \\
0 & 0 & \delta^{2} I
\end{array}\right]>\sum_{l \in \mathbb{M}_{i}} \alpha_{i l}\left[\begin{array}{ccc}
M_{i l}^{(\infty, 1,1)} & \bullet & \bullet \\
M_{i l}^{(\infty, 2,1)} & M_{i l}^{(\infty, 2,2)} & \bullet \\
N_{i l}^{(\infty, 1,1)} & N_{i l}^{(\infty, 2,1)} & S_{i l}^{(\infty)}
\end{array}\right]} \\
& {\left[\begin{array}{cccccc}
M_{i l}^{(\infty, 1,1)} & \bullet & \bullet & \bullet & \bullet & \bullet \\
M_{i l}^{(\infty, 2,1)} & M_{i l}^{(\infty, 2,2)} & \bullet & \bullet & \bullet & \bullet \\
N_{i l}^{(\infty, 1,1)} & N_{i l}^{(\infty, 2,1)} & S_{i l}^{(\infty)} & \bullet & \bullet & \bullet \\
\mathcal{E}_{i}(Z) A_{i} & \mathcal{E}_{i}(Z) A_{i} & \mathcal{E}_{i}(Z) J_{i} & \mathcal{E}_{i}(Z) & \bullet & \bullet \\
G_{l} A_{i}+F_{l} L_{i}+R_{l} & G_{l} A_{i}+F_{l} L_{i} & G_{l} J_{i}+F_{l} H_{i} & 0 & \mathcal{X}_{i l} & \bullet \\
C_{i}^{(\infty)}+K_{l} L_{i}+O_{l} & C_{i}^{(\infty)}+K_{l} L_{i} & E_{i}^{(\infty)}+K_{l} H_{i} & 0 & 0 & I
\end{array}\right]>0}
\end{aligned}
$$

where $\mathcal{X}_{i l}=\operatorname{Her}\left(G_{l}\right)+\mathcal{E}_{i}(Z)-\mathcal{E}_{i}(T)$. Consider also the following sets

$$
\begin{aligned}
& \xi_{(\infty, a)} \triangleq\left\{T_{i}, Z_{i} \text { for all } i \in \mathbb{N}\right\}, \\
& \xi_{(\infty, b)} \triangleq\left\{M_{i l}^{(\infty, 1,1)}, M_{i l}^{(\infty, 2,1)}, M_{i l}^{(\infty, 2,2)}, N_{i l}^{(\infty, 1,1)}, N_{i l}^{(\infty, 1,2)} S_{i l}^{(\infty)} \text { for all } i \in \mathbb{N}, l \in \mathbb{M}_{i}\right\},
\end{aligned}
$$

such that $\xi_{\infty} \triangleq \xi_{(\infty, a)} \cup \xi_{(f)} \cup \xi_{(\infty, b)}$, where $\xi_{(f)}$ is given in (5.11). The set of all possible solutions of (5.18)-(5.19) for a given $\delta$ is $\Xi_{\infty} \triangleq\left\{\xi_{\infty}\right.$ such that (5.18) - (5.19) hold $\}$. 
Lemma 5.2. Consider the following statements for a given $\delta>0$ :

(i) There exists $\xi_{\infty}$ such that $\xi_{\infty} \in \Xi_{\infty}$.

(ii) There exists $\mathcal{F} \in \mathbb{F}$ with $\left\|\mathcal{G}_{f}\right\|_{\infty}<\delta$.

We have that $($ ii $) \Longrightarrow$ (i) by setting $A_{f l}=-G_{l}^{-1} R_{l}, B_{f l}=-G_{l}^{-1} F_{l}, C_{f l}=-O_{l}$ and $D_{f l}=-K_{l}$ or all $l \in \mathbb{M}$. If $\hat{\theta}=\theta$, then $(i) \Longrightarrow(i i)$.

Proof. It follows the same lines as in the proof of Lemma 5.1. Considering that $\xi_{\infty} \in$ $\Xi_{\infty}$, setting $R_{l}=-G_{l} A_{f l}, F_{l}=-G_{l} B_{f l}, O_{l}=-C_{f l}$, and $K_{l}=-D_{f l}$, and recalling Lemma A.2, we get that

$$
\left[\begin{array}{cccccc}
M_{i l}^{(\infty, 1,1)} & \bullet & \bullet & \bullet & \bullet & \bullet \\
M_{i l}^{(\infty, 2,1)} & M_{i l}^{(\infty, 2,2)} & \bullet & \bullet & \bullet & \bullet \\
N_{i l}^{(\infty, 1,1)} & N_{i l}^{(\infty, 2,1)} & S_{i l}^{(\infty)} & \bullet & \bullet & \bullet \\
\mathcal{E}_{i}(Z) A_{i} & \mathcal{E}_{i}(Z) A_{i} & \mathcal{E}_{i}(Z) J_{i} & \mathcal{E}_{i}(Z) & \bullet & \bullet \\
G_{l}\left(A_{i}-B_{f l} L_{i}-A_{f l}\right) & G_{l}\left(A_{i}-B_{f l} L_{i}\right) & G_{l}\left(J_{i}-B_{f l} H_{i}\right) & 0 & \mathcal{X}_{i l}^{(2)} & \bullet \\
C_{i}^{(\infty)}-D_{f l} L_{i}-C_{f l} & C_{i}^{(\infty)}-D_{f l} L_{i} & E_{i}^{(\infty)}-B_{f l} H_{i} & 0 & 0 & I
\end{array}\right]>0,
$$

where $\left.\mathcal{X}_{i l}^{(2)} \triangleq G_{l}\left[\mathcal{E}_{i}(T)-\mathcal{E}_{i}(Z)\right)^{-1}\right] G_{l}^{\prime}$. Defining the matrix

$$
\mathcal{D}_{i l}^{(\infty)}=\left[\begin{array}{cc}
I & I \\
0 & G_{l}^{-T}\left[\mathcal{E}_{i}(Z)-\mathcal{E}_{i}(T)\right]
\end{array}\right],
$$

and applying the congruence transformation $\operatorname{diag}\left(I_{r}, \mathcal{D}_{i l}^{(\infty)}, I_{q_{\infty}}\right)$ to $(5.20)$ yields

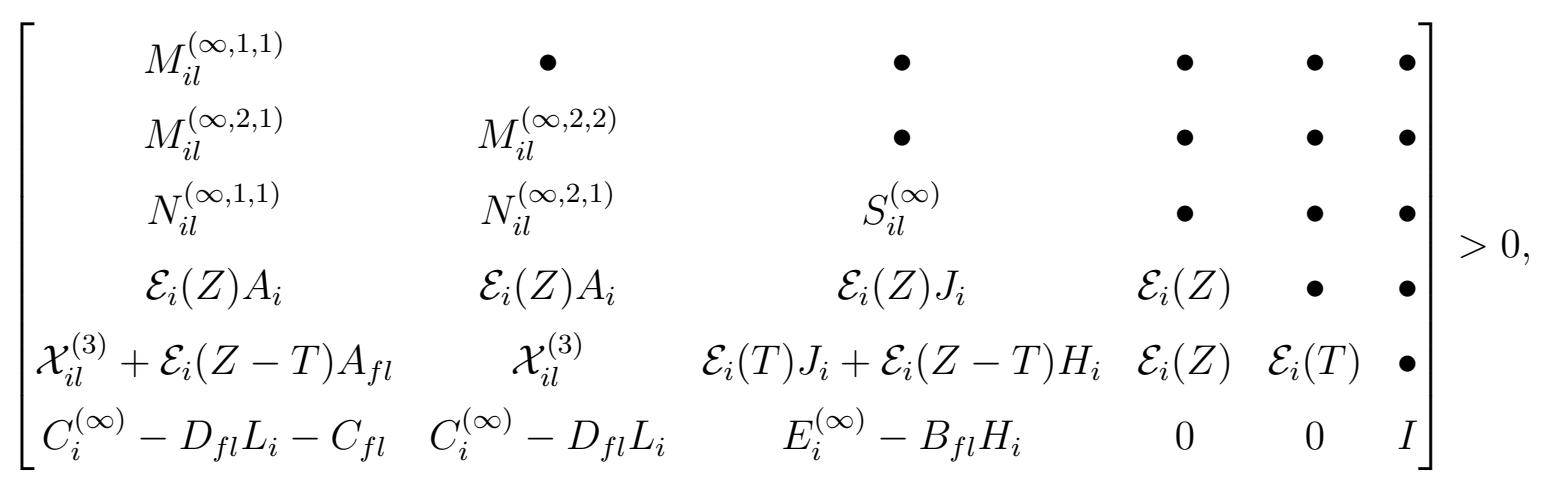

where $\mathcal{X}_{i l}^{(3)} \triangleq \mathcal{E}_{i}(Z) A_{i}+\mathcal{E}_{i}(Z-T) B_{f l} L_{i}$. By restricting the structure of $Q_{i}$ in (3.26) as in (A.2) with $\bar{U}_{i}=\bar{T}_{i}$, we get that $\bar{V}_{i} \bar{T}_{i}=I, Z_{i}=T_{i}+\bar{U}_{i}$, and $\hat{Q}_{1 i}^{-1}=\mathcal{E}_{i}(Z)$. Thus, we are 
able to write (5.21) as follows

$$
\left[\begin{array}{cccc}
\mathcal{T}_{i}^{(\infty))^{\prime}} M_{i l}^{(\infty)} \mathcal{T}_{i}^{(\infty)} & \bullet & \bullet & \bullet \\
N_{i l}^{(\infty)} \mathcal{T}_{i}^{(\infty)} & S_{i l}^{(\infty)} & \bullet & \bullet \\
\mathcal{H}_{i}^{(\infty)^{\prime}} A_{i l}^{(\infty)} \mathcal{T}_{i}^{(\infty)} & \mathcal{H}_{i}^{(\infty)^{\prime}} J_{i l}^{(\infty)} & \mathcal{H}_{i}^{(\infty)^{\prime}} \mathcal{E}_{i}(Q)^{-1} \mathcal{H}_{i}^{(\infty)} & \bullet \\
C_{i l}^{(\infty)} \mathcal{T}_{i}^{(\infty)} & E_{i l}^{(\infty)} & 0 & I
\end{array}\right]>0
$$

for the systems matrices given in (5.4), and $\mathcal{T}_{i}^{(\infty)}$ and $\mathcal{H}_{i}^{(\infty)}$ given in (A.3) and (A.5), respectively. Additionally (5.18) can be rewritten as

$$
\left[\begin{array}{cc}
\mathcal{T}_{i}^{(\infty)^{\prime}} Q_{i} \mathcal{T}_{i}^{(\infty)} & \bullet \\
0 & \delta^{2} I
\end{array}\right]>\sum_{l \in \mathbb{M}_{i}} \alpha_{i l}\left[\begin{array}{cc}
\mathcal{T}_{i}^{(\infty)^{\prime}} M_{i l}^{(\infty)} \mathcal{T}_{i}^{(\infty)} & \bullet \\
N_{i l}^{(\infty)} \mathcal{T}_{i}^{(\infty)} & S_{i l}^{(\infty)}
\end{array}\right]
$$

Applying the congruence transformations $\operatorname{diag}\left(\mathcal{T}_{i}^{(\infty)^{-1}}, I_{r}, \mathcal{H}_{i}^{(\infty)^{-1}}, I_{q_{\infty}}\right)$ and $\operatorname{diag}\left(\mathcal{T}_{i}^{(\infty)^{-1}}, I_{q_{\infty}}\right)$ yields (3.26) with system matrices in (5.4).

Conversely, if $\hat{\theta}=\theta$, we get that $\alpha_{i i}=1$ for all $i \in \mathbb{N}$. By applying a suitable perturbation $\epsilon_{i}>0$ to (16) of (GONÇALVES; FIORAVANTI; GEROMEL, 2010) such that it still holds, and defining

$$
\left[\begin{array}{ccc}
Z_{i}-\epsilon_{i} I & \bullet & \bullet \\
Z_{i} & T_{i}-\epsilon_{i} I & \bullet \\
0 & 0 & \delta^{2} I-\epsilon_{i} I
\end{array}\right]<\left[\begin{array}{ccc}
M_{i i}^{(\infty, 1,1)} & \bullet & \bullet \\
M_{i i}^{(\infty, 2,1)} & M_{i i}^{(\infty, 2,2)} & \bullet \\
N_{i i}^{(\infty, 1,1)} & N_{i i}^{(\infty, 2,1)} & S_{i i}^{(\infty)}
\end{array}\right]<\left[\begin{array}{ccc}
Z_{i} & \bullet & \bullet \\
Z_{i} & T_{i} & \bullet \\
0 & 0 & \delta^{2} I
\end{array}\right]
$$

we get (5.18)-(5.19).

By defining the following set $\bar{\xi}_{\infty} \triangleq \xi_{\infty} \cup\left\{\delta_{\text {aux }}\right\}$, where $\delta_{\text {aux }} \triangleq \delta^{2}$, and considering the set of all possible solutions of (5.18)-(5.19) given by $\bar{\Xi}_{(\infty)} \triangleq\left\{\bar{\xi}_{\infty}\right.$ such that (5.18) (5.19) holds $\}$, we write the $\mathcal{H}_{\infty}$ filtering for hidden MJLS as follows:

$$
\inf _{\bar{\xi}_{\infty} \in \bar{\Xi}_{\infty}} \delta_{\text {aux }}
$$

Example 5.2 $\mathcal{H}_{\infty}$ filtering for hidden MJLS: We use the system matrices of Example 5.1 , along with

$$
C_{i}^{(\infty)}=\left[\begin{array}{ll}
0 & 1 \\
0 & 0
\end{array}\right], \quad E_{i}^{(\infty)}=0_{2 \times 2}
$$

for all $i \in \mathbb{N}$, that is, we want the best estimate of $x_{2}(k)$ if $w \in l_{2}^{r}(\Omega, \mathfrak{F}$, Prob). By setting 
$\rho_{1}=\rho_{2}=1$ in (5.17) and solving (5.22), we get

$$
\left[\begin{array}{c|c}
A_{f 1} & B_{f 1} \\
\hline C_{f 1} & D_{f 1}
\end{array}\right]=\left[\begin{array}{rr|r}
-0.8725 & -0.5000 & 0.8725 \\
1.7450 & 1.0000 & -0.7450 \\
\hline 1.7450 & 1.0000 & -1.7450 \\
0 & 0 & 0
\end{array}\right]
$$

and

$$
\left[\begin{array}{c|c}
A_{f 2} & B_{f 2} \\
\hline C_{f 2} & D_{f 2}
\end{array}\right]=\left[\begin{array}{rr|r}
-0.3567 & -0.3300 & 0.3567 \\
1.5135 & 1.4000 & -0.5135 \\
\hline 1.0810 & 1.0000 & -1.0810 \\
0 & 0 & 0
\end{array}\right],
$$

with $\delta^{*}=\left\|\mathcal{G}_{*}\right\|_{\infty}=3.3912$. Conversely, we set $\rho_{1}=\rho_{2}=0.7$ and solve (5.22) in order to get

$$
\left[\begin{array}{c|c}
A_{f 1} & B_{f 1} \\
\hline C_{f 1} & D_{f 1}
\end{array}\right]=\left[\begin{array}{rc|c}
-0.6300 & -0.3125 & 1.3553 \\
1.3166 & 0.6734 & -1.6595 \\
\hline 1.6436 & 0.9573 & -1.6080 \\
0 & 0 & 0
\end{array}\right]
$$

and

$$
\left[\begin{array}{c|c}
A_{f 2} & B_{f 2} \\
\hline C_{f 2} & D_{f 2}
\end{array}\right]=\left[\begin{array}{rr|r}
-0.7131 & -0.3860 & 0.5620 \\
1.4882 & 0.8165 & -0.8103 \\
\hline 1.8737 & 1.0913 & -1.8458 \\
0 & 0 & 0
\end{array}\right],
$$

with $\delta^{*}=3.5994$ and $\left\|\mathcal{G}_{f}\right\|_{\infty}=3.5981$.

\subsection{The mixed $\mathcal{H}_{2} / \mathcal{H}_{\infty}$ filtering for hidden MJLS}

Similarly to what we have done in Chapter 4, Lemmas 5.1 and 5.2 allow us to state a theorem that presents conditions for achieving mixed $\mathcal{H}_{2} / \mathcal{H}_{\infty}$ filters. For that, define $\bar{\xi} \triangleq \xi_{2} \cup \xi_{\infty} \cup \xi_{\text {aux }}$, where $\xi_{\text {aux }}$ is the set that contains $\delta, \gamma$ or both depending on the 
objective function $g(\gamma, \delta)$. Define also

$\bar{\Xi} \triangleq\{\xi$ such that the LMls $(5.7)-(5.10)$ and $(5.18)-(5.19)$ are jointly feasible $\}$.

Theorem 5.1. If there exists $\bar{\xi} \in \bar{\Xi}$, then by setting $A_{f l}=-G_{l}^{-1} R_{l}, B_{f l}=-G_{l}^{-1} F_{l}, C_{f l}=$ $-O_{l}$, and $D_{f l}=-K_{l}$ for all $l \in \mathbb{M}$, we have that $\mathcal{F} \in \mathbb{F},\left\|\mathcal{G}_{f}\right\|_{2}<\gamma$, and $\left\|\mathcal{G}_{f}\right\|_{\infty}<\delta$.

Proof. The result is a direct consequence of Lemmas 5.1 and 5.2 and the use of the same variables $G_{l}, R_{l}, F_{l}, O_{l}$, and $K_{l}$ that are used for calculating the filter matrices.

In this case, we rewrite the optimization problem (5.6) as follows:

$$
\inf _{\xi \in \Xi} g(\gamma, \delta) .
$$

Remark 5.2: We can simplify the result of Theorem 5.1 by exploiting the properties shown in Corollary 3.2. Then, we set $C_{i}^{(2)}=C_{i}^{(\infty)}=C_{i}, D_{i}^{(2)}=D_{i}^{(\infty)}=D_{i}, Y_{i}=Z_{i}$, and $X_{i}=T_{i}$. Theorem 5.1 still holds if we redefine $\bar{\xi}=\xi_{\infty} \cup\left\{W_{i l}, i \in \mathbb{N}, l \in M_{i}\right\}$, and $\bar{\Xi}=\{\bar{\xi}$ such that the LMls (5.7) - (5.8) and (5.18) - (5.19) are jointly feasible $\}$.

Example 5.3 The mixed $\mathcal{H}_{2} / \mathcal{H}_{\infty}$ filtering for hidden MJLS: We use the system matrices of Example 5.1 and 5.2. The interpretation is, if $w$ is a wide-sense white noise sequence, we want the best estimate of $x_{1}(k)$ and $x_{2}(k)$, and if $w$ is a sequence of finite energy, we want an estimate of $x_{2}(k)$ that is robust to this type of exogenous input. We set $\rho_{1}=\rho_{2}=0.7$ and $\delta=4$ to solve (5.23) for $g(\gamma, \delta)=\gamma^{2}$. The filter matrices are given below

$$
\left[\begin{array}{l|l}
A_{f 1} & B_{f 1} \\
\hline C_{f 1} & D_{f 1}
\end{array}\right]=\left[\begin{array}{rr|r}
-1.4829 & -1.1835 & 0.8376 \\
2.3676 & 1.8252 & -0.8830 \\
\hline 0.2546 & 0.1972 & -0.6689 \\
0.5030 & 0.3847 & -0.1600
\end{array}\right]
$$

and

$$
\left[\begin{array}{l|l}
A_{f 2} & B_{f 2} \\
\hline C_{f 2} & D_{f 2}
\end{array}\right]=\left[\begin{array}{rr|r}
-0.8730 & -0.6423 & 0.6763 \\
1.9743 & 1.5008 & -1.0511 \\
\hline 0.1415 & 0.1123 & -0.7322 \\
0.0895 & 0.0789 & -0.1852
\end{array}\right],
$$

with $\gamma^{*}=1.8496,\left\|\mathcal{G}_{f}\right\|_{2}=1.8442$, and $\left\|\mathcal{G}_{f}\right\|_{\infty}=3.9939$. 


\subsection{Filtering for the cluster case and robust filtering}

In this section, we study the equivalence between the filtering problem for hidden MJLS under Assumption 3.1 and the cluster case of (GONÇALVES; FIORAVANTI; GEROMEL, 2010). Since we use different Lyapunov matrices in the $\mathcal{H}_{2}$ and $\mathcal{H}_{\infty}$ filtering, we transcribe the LMIs from (GONÇALVES; FIORAVANTI; GEROMEL, 2010) for the cluster case modified to our purposes. Consider the following matrix inequality set for all $s \in\{1, \ldots, \kappa\}, i \in \mathbb{N}^{s}$,

$$
\begin{aligned}
& \sum_{i \in \mathbb{N}} \mu_{i} \operatorname{Tr}\left(\bar{W}_{i}\right)<\gamma^{2}, \\
& {\left[\begin{array}{cccc}
\bar{W}_{i} & \bullet & \bullet & \bullet \\
\mathcal{E}_{i}(Y) J_{i} & \mathcal{E}_{i}(Y) & \bullet & \bullet \\
G^{s} J_{i}+F^{s} H_{i} & 0 & \operatorname{Her}\left(G^{s}\right)+\mathcal{E}_{i}(Y)-\mathcal{E}_{i}(X) & \bullet \\
E_{i}^{(2)}+K^{s} H_{i} & 0 & 0 & I
\end{array}\right]>0,}
\end{aligned}
$$

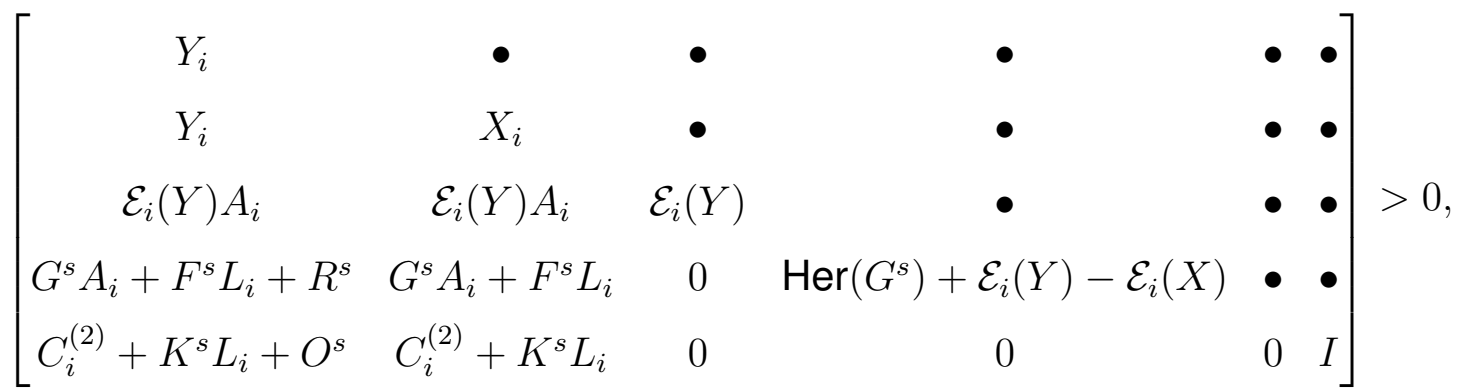

as well as

$$
\left[\begin{array}{cccccc}
Z_{i} & \bullet & \bullet & \bullet & \bullet & \bullet \\
Z_{i} & T_{i} & \bullet & \bullet & \bullet & \bullet \\
0 & 0 & \delta^{2} I & \bullet & \bullet & \bullet \\
\mathcal{E}_{i}(Z) A_{i} & \mathcal{E}_{i}(Z) A_{i} & \mathcal{E}_{i}(Z) J_{i} & \mathcal{E}_{i}(Z) & \bullet & \bullet \\
G^{s} A_{i}+F^{s} L_{i}+R^{s} & G^{s} A_{i}+F^{s} L_{i} & G^{s} J_{i}+F^{s} H_{i} & 0 & \mathcal{X}_{i l} & \bullet \\
C_{i}^{(\infty)}+K^{s} L_{i}+O^{s} & C_{i}^{(\infty)}+K^{s} L_{i} & E_{i}^{(\infty)}+K^{s} H_{i} & 0 & 0 & I
\end{array}\right]>0
$$

where $\mathcal{X}_{i l} \triangleq \operatorname{Her}\left(G^{s}\right)+\mathcal{E}_{i}(Z-T)$. We have the following proposition.

Proposition 5.1: Given that Assumption 3.1 holds, the following statements also hold true: 
- Conditions (5.7)-(5.10) are equivalent to (5.24)-(5.25).

- Conditions (5.18)-(5.19) are equivalent to (5.27).

Proof. It follows the similar steps as in the proof of Proposition 4.1.

We now suppose that $\mathbb{P}$ and $\Upsilon$ are properly described by (3.3) and (3.4), respectively, such that $\mathbb{P} \in \mathbb{D}_{\mathbb{P}}$ and $\Upsilon \in \mathbb{D}_{\Upsilon}$. We define the set $\mathbb{F}_{r} \triangleq\{\mathcal{F}:(5.3)$ for $\mathbb{P} \in$ $\mathbb{D}_{\mathbb{P}}$ and $\Upsilon \in \mathbb{D}_{\Upsilon}$ is $\left.S S\right\}$, and set

$$
\begin{aligned}
\alpha_{i l}=\alpha_{i l}^{(t)}, & \mathcal{E}_{i}(Y)=\mathcal{E}_{i}^{(h)}(Y), & \mathcal{E}_{i}(X)=\mathcal{E}_{i}^{(h)}(X), \\
& \mathcal{E}_{i}(T)=\mathcal{E}_{i}^{(h)}(T), & \mathcal{E}_{i}(Z)=\mathcal{E}_{i}^{(h)}(Z),
\end{aligned}
$$

in (5.7)-(5.10) and (5.18)-(5.19), where $\mathcal{E}_{i}^{(h)}(U) \triangleq \sum_{j \in \mathbb{N}} p_{i j}^{(h)} U_{j}$. By defining $\bar{\Xi}^{(u)}=$ $\{\bar{\xi} \quad ; \quad$ such that $(5.7)-(5.10)$ and $(5.18)-(5.19)$ subject to (5.28) hold for all $h \in$ $\left.\{1, \ldots, \eta\}, t \in\{1, \ldots, \eta\}, i \in \mathbb{N}, l \in \mathbb{M}_{i}\right\}$, we have the following theorem regarding the synthesis of filters for $\mathbb{P} \in \mathbb{D}_{\mathbb{P}}$ and $\Upsilon \in \mathbb{D}_{\Upsilon}$.

Theorem 5.2. Given that $\mathbb{P} \in \mathbb{D}_{\mathbb{P}}$ and $\Upsilon \in \mathbb{D}_{\Upsilon}$, if $\bar{\xi} \in \bar{\Xi}^{(u)}$, then by setting $A_{f l}=$ $-G_{l}^{-1} R_{l}, B_{f l}=-G_{l}^{-1} F_{l}, C_{f l}=-O_{l}$ and $D_{f l}=-K_{l}$ for all $l \in \mathbb{M}$, we have that $\mathcal{F} \in \mathbb{F}_{r}$, $\left\|\mathcal{G}_{f}\right\|_{2}<\gamma$, and $\left\|\mathcal{G}_{f}\right\|_{\infty}<\delta$.

Proof. It follows by noting that (5.7)-(5.10) and (5.18)-(5.19) subject to (5.28) are affine with respect to $\alpha_{i l}^{(t)}$ and $p_{i j}^{(h)}$, and so by multiplying the inequalities by $a_{h}$ and $b_{t}$ and summing them up for all $s \in\{1, \ldots, \eta\}$ and $t \in\{1, \ldots, \eta\}$, we get the original conditions.

Example 5.4 The mixed $\mathcal{H}_{2} / \mathcal{H}_{\infty}$ filtering for the cluster case: We now set $\rho_{1}=$ $\rho_{2}=0.5$ and solve (5.23) for $g(\gamma, \delta)=\gamma^{2}$ and $\delta=5$. The resulting mixed $\mathcal{H}_{2} / \mathcal{H}_{\infty}$ mode-independent filter is given below:

$$
\left[\begin{array}{c|c}
A_{f i} & B_{f i} \\
\hline C_{f i} & D_{f i}
\end{array}\right]=\left[\begin{array}{rr|r}
-0.7083 & -0.2706 & 0.1584 \\
0.8602 & 0.3456 & -0.0554 \\
\hline 0.5904 & 0.4724 & 0.3371 \\
0.4349 & 0.3926 & -0.3397
\end{array}\right],
$$

for $i \in \mathbb{N}$, with $\gamma^{*}=1.2985,\left\|\mathcal{G}_{f}\right\|_{2}=1.2981$, and $\left\|\mathcal{G}_{f}\right\|_{\infty}=5.2746$. 


\subsection{Filtering for the Bernoulli case for hidden MJLS}

In this section we will briefly analyze the separated problems of the optimal $\mathcal{H}_{2}$ and $\mathcal{H}_{\infty}$ filtering for the Bernoulli case. The mixed formulation could also be tackled at the expense of a common Lyapunov matrix for both problems. Since that leads to more conservative results, we do not address it directly here.

Consider the following inequality set, for all $i \in \mathbb{N}, l \in \mathbb{M}_{i}$, and a given $\gamma>0$ :

$$
\begin{aligned}
& \sum_{i \in \mathbb{N}} \sum_{l \in \mathbb{M}_{i}} \mu_{i} \alpha_{i l} \operatorname{Tr}\left(W_{i l}\right)<\gamma^{2}, \\
& {\left[\begin{array}{cccc}
W_{i l} & \bullet & \bullet & \bullet \\
Y J_{i} & Y & \bullet & \bullet \\
X J_{i}+F_{l} H_{i} & Y & X & \bullet \\
E_{i}^{(2)}+K_{l} H_{i} & 0 & 0 & I
\end{array}\right]>0,} \\
& {\left[\begin{array}{cc}
Y & Y \\
Y & X
\end{array}\right]>\sum_{i \in \mathbb{N}} \sum_{l \in \mathbb{M}_{i}} p_{i} \alpha_{i l}\left[\begin{array}{cccc}
M_{i l}^{(2,1,1)} & \bullet \\
M_{i l}^{(2,2,1)} & M_{i l}^{(2,2,2)}
\end{array}\right],} \\
& {\left[\begin{array}{ccccc}
M_{i l}^{(2,1,1)} & \bullet & \bullet & \bullet & \bullet \\
M_{i l}^{(2,2,1)} & M_{i l}^{(2,2,2)} & \bullet & \bullet & \bullet \\
Y A_{i} & Y A_{i} & Y & \bullet & \bullet \\
X A_{i}+F_{l} L_{i}+R_{l} & X A_{i}+F_{l} L_{i} & Y & X & \bullet \\
C_{i}^{(2)}+K_{l} L_{i}+O_{l} & C_{i}^{(2)}+K_{l} L_{i} & 0 & 0 & I
\end{array}\right]>0,}
\end{aligned}
$$

for all $i \in \mathbb{N}, l \in \mathbb{M}_{i}$. For $\xi_{2} \triangleq\left\{Y, X, M_{i l}^{(2,1,1)}, M_{i l}^{(2,2,1)}, M_{i l}^{(2,2,2)}, i \in \mathbb{N}, l \in\right.$ $\left.\mathbb{M}_{i}, R_{l}, F_{l}, K_{l}, O_{l}, l \in \mathbb{M}\right\}, \Xi_{2} \triangleq\left\{\xi_{2}\right.$ such that (5.29) - (5.32) holds $\}$, we have the following theorem.

Theorem 5.3. There exists a filter such that $\mathcal{F} \in \mathbb{F}$ with $\left\|\mathcal{G}_{f}\right\|_{2}<\gamma$, for a given $\gamma>0$, if and only if there is some $\xi_{2}$ such that $\xi_{2} \in \Xi_{2}$. In the affirmative case, the filter matrices can be calculated by setting $A_{f l}=(Y-X)^{-1} R_{l}, B_{f l}=(Y-X)^{-1} F_{l}, C_{f l}=-O_{l}$ and $D_{f l}=-K_{l}$ for all $l \in \mathbb{M}$.

Proof. By means of the Schur complement (see Lemma A.1 in the Appendix A) and of 
additional auxiliary variables, we can rewrite the conditions of (3.5) as follows

$$
\begin{gathered}
\sum_{i \in \mathbb{N}} \sum_{l \in \mathbb{M}_{i}} \mu_{i} \alpha_{i l} \operatorname{Tr}\left(W_{i l}\right)<\gamma^{2}, \\
{\left[\begin{array}{lll}
W_{i l} & \bullet & \bullet \\
\bar{P} J_{i l} & \bar{P} & \bullet \\
E_{i l}^{(2)} & 0 & i I
\end{array}\right]>0,} \\
\bar{P}>\sum_{i \in \mathbb{N}} \sum_{l \in \mathbb{M}_{i}} p_{i} \alpha_{i l} M_{i l}^{(2)}, \\
{\left[\begin{array}{lll}
M_{i l}^{(2)} & \bullet & \bullet \\
\bar{P} A_{i l} & \bar{P} & \bullet \\
C_{i l}^{(2)} & 0 & I
\end{array}\right]>0,}
\end{gathered}
$$

for all $i \in \mathbb{N}, l \in \mathbb{M}_{i}$, and then we get that $\left\|\mathcal{G}_{f}\right\|_{2}^{2}=\inf _{W_{i l}, \bar{P}} \gamma^{2}$ subject to (5.33)-(5.36) for a $\mathcal{F} \in \mathbb{F}$. Consider the following partition of $\bar{P}$ in (3.22)

$$
\bar{P}=\left[\begin{array}{cc}
X & \hat{U} \\
\hat{U}^{\prime} & \hat{X}
\end{array}\right], \bar{P}^{-1}=\left[\begin{array}{cc}
Y^{-1} & \hat{V} \\
\hat{V}^{\prime} & \hat{Y}
\end{array}\right],
$$

and define

$$
\mathcal{T}^{(2)}=\left[\begin{array}{cc}
I & I \\
\hat{V}^{\prime} Y & 0
\end{array}\right]
$$

such that

$$
\mathcal{T}^{(2)^{\prime}} \bar{P} \mathcal{T}^{(2)}=\left[\begin{array}{ll}
Y & Y \\
Y & X
\end{array}\right] .
$$

It is then direct that applying the congruence transformations $\operatorname{diag}\left(I_{r}, \mathcal{T}^{(2)}, I_{q_{2}}\right), \mathcal{T}^{(2)}$, and $\operatorname{diag}\left(\mathcal{T}^{(2)}, \mathcal{T}^{(2)}, I_{q_{2}}\right)$ to (5.34)-(5.36), respectively, and defining $R_{l} \triangleq \hat{U}^{\prime} A_{f l} \hat{V}^{\prime} Y, F_{l} \triangleq$ $\hat{U} B_{f l}, O_{l} \triangleq=-C_{f l} \hat{V}^{\prime} Y$, and $K_{L} \triangleq-D_{f l}$, we get (5.29)-(5.32). Conversely, by fixing the structure of $\bar{P}$ as follows

$$
\bar{P}=\left[\begin{array}{cc}
X & Y-X \\
Y-X & X-Y
\end{array}\right],
$$

we get that $U=Y-X$ and $\hat{V}^{\prime} Y=Y \hat{V}=I$. It readily follows that by setting $A_{f l}=$ $(Y-X) R_{l}, B_{f l}=(Y-X) F_{l}, C_{f l}=-O_{l}$, and $D_{f l}=-K_{l}$ for all $l \in \mathbb{M}$, and applying 
the congruence transformations $\operatorname{diag}\left(I_{r}, \mathcal{T}^{(2)^{-1}}, I_{q_{2}}\right), \mathcal{T}^{(2)^{-1}}$, and $\operatorname{diag}\left(\mathcal{T}^{(2)^{-1}}, \mathcal{T}^{(2)^{-1}}, I_{q_{2}}\right)$ to (5.30)-(5.32), we get (5.34)-(5.36).

Setting $\bar{\xi}_{2}=\xi_{2} \cup\left\{\gamma_{a u x}\right\}, \gamma_{a u x}=\gamma^{2}$, and considering $\bar{\Xi}_{2}=\left\{\bar{\xi}_{2} ;\right.$ such that (5.29) (5.32) hold\}, we have that

$$
\left\|\mathcal{G}_{*}\right\|_{2}^{2}=\inf _{\bar{\xi}_{2} \in \bar{\Xi}_{2}} \gamma_{\text {aux }}
$$

We now study the problem of the $\mathcal{H}_{\infty}$ filtering problem for the Bernoulli condition. Consider the following inequalities for all $i \in \mathbb{N}, l \in \mathbb{M}_{i}$, and a given $\delta>0$ :

$$
\begin{aligned}
& {\left[\begin{array}{cc}
Z & \bullet \\
Z & T
\end{array}\right]>\sum_{i \in \mathbb{N}} p_{i}\left[\begin{array}{cc}
Q_{i}^{(1,1)} & \bullet \\
Q_{i}^{(2,1)} & Q_{i}^{(2,2)}
\end{array}\right]} \\
& {\left[\begin{array}{ccc}
Q_{i}^{(1,1)} & \bullet & \bullet \\
Q_{i}^{(2,1)} & Q_{i}^{(2,2)} & \bullet \\
0 & 0 & \delta^{2} I
\end{array}\right]>\sum_{l \in \mathbb{M}_{i}} \alpha_{i l}\left[\begin{array}{ccc}
M_{i l}^{(\infty, 1,1)} & \bullet & \bullet \\
M_{i l}^{(\infty, 2,1)} & M_{i l}^{(\infty, 2,2)} & \bullet \\
N_{i l}^{(\infty, 1,1)} & N_{i l}^{(\infty, 1,2)} & S_{i l}^{(\infty, 1,1)}
\end{array}\right] \text {, }} \\
& {\left[\begin{array}{cccccc}
M_{i l}^{(\infty, 1,1)} & \bullet & \bullet & \bullet & \bullet & \bullet \\
M_{i l}^{(\infty, 2,1)} & M_{i l}^{(\infty, 2,2)} & \bullet & \bullet & \bullet & \bullet \\
N_{i l}^{(\infty, 1,1)} & N_{i l}^{(\infty, 1,2)} & S_{i l}^{(\infty, 1,1)} & \bullet & \bullet & \bullet \\
Z A_{i} & Z A_{i} & Z J_{i} & Z & \bullet & \bullet \\
T A_{i}+F_{l} L_{i}+R_{l} & T A_{i}+F_{l} L_{i} & T J_{i}+F_{l} H_{i} & Z & T & \bullet \\
C_{i}^{(\infty)}+K_{l} L_{i}+O_{l} & C_{i}^{(\infty)}+K_{l} L_{i} & E_{i}^{(\infty)}+K_{l} H_{i} & 0 & 0 & I
\end{array}\right]>0 .}
\end{aligned}
$$

For the following sets

$$
\begin{aligned}
& \xi_{(\infty, 1)} \triangleq\left\{Q_{i}^{(11)}, Q_{i}^{(21)}, Q_{i}^{(22)} \text { for all } i \in \mathbb{N}\right\}, \\
& \xi_{(\infty, 2)} \triangleq\left\{M_{i l}^{(\infty, 1,1)}, M_{i l}^{(\infty, 2,1)}, M_{i l}^{(\infty, 2,2)}, N_{i l}^{(\infty, 1,1)}, N_{i l}^{(\infty, 1,2)}, S_{i l}^{(\infty)} \text { for all } i \in \mathbb{N}, l \in \mathbb{M}_{i}\right\},
\end{aligned}
$$

such that $\xi_{\infty} \triangleq \xi_{(\infty, 1)} \cup \xi_{(\infty, 2)} \cup\left\{R_{l}, F_{l}, O_{l}, K_{l}, l \in \mathbb{M}\right\}$, as well as, $\Xi_{\infty} \triangleq$ $\left\{\xi_{\infty}\right.$ such that $(5.38)-(5.40)$ holds $\}$. We have the following result.

Theorem 5.4. There exists a filter such that $\mathcal{F} \in \mathbb{F}$ with $\left\|\mathcal{G}_{f}\right\|_{\infty}<\delta$, for a given $\delta>0$, if and only if $\xi_{\infty} \in \Xi_{\infty}$. In the affirmative case, the filter matrices can be calculated by setting $A_{f l}=(Z-T)^{-1} R_{l}, B_{f l}=(Z-T)^{-1} F_{l}, C_{f l}=-O_{l}$ and $D_{f l}=-K_{l}$ for all $l \in \mathbb{M}$. 
Proof. We rewrite the conditions of (3.29)-(3.30) as follows:

$$
\begin{aligned}
& \bar{Q}>\sum_{i} p_{i} \bar{G}_{i}, \\
& {\left[\begin{array}{cc}
\bar{G}_{i} & \bullet \\
0 & \delta^{2} I
\end{array}\right]>\sum_{l \in \mathbb{M}_{i}} \alpha_{i l}\left[\begin{array}{cc}
M_{i l}^{(\infty)} & \bullet \\
N_{i l}^{(\infty)} & S_{i l}^{(\infty)}
\end{array}\right],} \\
& {\left[\begin{array}{cccc}
M_{i l}^{(\infty)} & \bullet & \bullet & \bullet \\
N_{i l}^{(\infty)} & S_{i l}^{(\infty)} & \bullet & \bullet \\
\bar{Q} A_{i l} & \bar{Q} J_{i l} & \bar{Q} & \bullet \\
C_{i l}^{(\infty)} & E_{i l}^{(\infty)} & 0 & I
\end{array}\right]>0 .}
\end{aligned}
$$

Consider the following partition of $\bar{P}$ in (3.22)

$$
\bar{Q}=\left[\begin{array}{cc}
T & \bar{U} \\
\bar{U}^{\prime} & \hat{T}
\end{array}\right], \bar{Q}^{-1}=\left[\begin{array}{cc}
Z^{-1} & \bar{V} \\
\bar{V}^{\prime} & \hat{Z}
\end{array}\right],
$$

and define

$$
\mathcal{T}^{(\infty)}=\left[\begin{array}{cc}
I & I \\
\bar{V}^{\prime} Z & 0
\end{array}\right]
$$

such that

$$
\mathcal{T}^{(\infty)^{\prime}} \bar{Q} \mathcal{T}^{(\infty)}=\left[\begin{array}{ll}
Z & Z \\
Z & T
\end{array}\right] .
$$

Then, the proof follows along the lines of the proof of Theorem 5.3, mutatis mutandis.

Setting $\bar{\xi}_{\infty} \triangleq \xi_{\infty} \cup\left\{\delta_{\text {aux }}\right\}, \delta_{\text {aux }}=\delta^{2}$, and considering $\bar{\Xi}_{\infty} \triangleq\left\{\bar{\xi}_{\infty} ;\right.$ such that (5.38) (5.40) hold\}, we write the following optimization problem for the $\mathcal{H}_{\infty}$ filtering under the Bernoulli condition:

$$
\left\|\mathcal{G}_{*}\right\|_{\infty}=\inf _{\bar{\xi}_{\infty} \in \bar{\Xi}_{\infty}} \delta_{\text {aux }} .
$$

Example 5.5 $\mathcal{H}_{2}$ and $\mathcal{H}_{\infty}$ filtering in the Bernoulli setting: We use the same system as of the previous examples, with exception of

$$
\mathbb{P}=\left[\begin{array}{ll}
2 / 3 & 1 / 3 \\
2 / 3 & 1 / 3
\end{array}\right] .
$$


By setting $\rho_{1}=\rho_{2}=0.7$, we get by solving (5.37)

$$
\left[\begin{array}{l|l}
A_{f 1} & B_{f 1} \\
\hline C_{f 1} & D_{f 1}
\end{array}\right]=\left[\begin{array}{rr|r}
-0.1056 & -0.4700 & 0.1056 \\
0.9415 & 1.0706 & 0.0585 \\
\hline 0.7008 & -0.0000 & 0.2992 \\
0.2248 & 1.0000 & -0.2248
\end{array}\right]
$$

and

$$
\left[\begin{array}{l|l}
A_{f 2} & B_{f 2} \\
\hline C_{f 2} & D_{f 2}
\end{array}\right]=\left[\begin{array}{rr|r}
-0.0918 & -0.4085 & 0.0918 \\
0.9740 & 1.2154 & 0.0260 \\
\hline 0.7008 & 0.0000 & 0.2992 \\
0.2248 & 1.0000 & -0.2248
\end{array}\right]
$$

with $\gamma^{*}=\left\|\mathcal{G}_{*}\right\|_{2}=1.0799$.

For the $\mathcal{H}_{\infty}$ filtering, we solve (5.44) in order to get

$$
\left[\begin{array}{c|c}
A_{f 1} & B_{f 1} \\
\hline C_{f 1} & D_{f 1}
\end{array}\right]=\left[\begin{array}{rr|r}
-0.4392 & -0.4762 & 0.5173 \\
1.0458 & 1.0719 & -0.0458 \\
\hline 0.9597 & 1.0272 & -0.9597 \\
0 & 0 & 0
\end{array}\right]
$$

and

$$
\left[\begin{array}{c|c}
A_{f 2} & B_{f 2} \\
\hline C_{f 2} & D_{f 2}
\end{array}\right]=\left[\begin{array}{rr|r}
-0.2781 & -0.3072 & 0.3561 \\
1.3777 & 1.2014 & -0.3777 \\
\hline 1.0456 & 0.9613 & -1.0456 \\
0 & 0 & 0
\end{array}\right]
$$

with $\delta^{*}=\left\|\mathcal{G}_{*}\right\|_{\infty}=1.9994$.

\subsection{Final remarks}

In this chapter, we studied the filtering problem for hidden MJLS. We derived conditions for the $\mathcal{H}_{2}, \mathcal{H}_{\infty}$, and mixed $\mathcal{H}_{2} / \mathcal{H}_{\infty}$ filtering considering that we have access only to $\hat{\theta}(k)$. We also analysed the cluster case and the robust filtering with respect to the transition and conditional probabilities. Finally, we considered the Bernoulli situation, in which we derived necessary and sufficient conditions for the $\mathcal{H}_{2} / \mathcal{H}_{\infty}$ filtering 
for hidden MJLS. Our results can be found in (OLIVEIRA; COSTA, 2017a; OLIVEIRA; COSTA, 2016b; OLIVEIRA; COSTA, 2017b).

As future steps, an interesting question is if it is possible to derive the Bernoulli design conditions for obtaining mixed $\mathcal{H}_{2} / \mathcal{H}_{\infty}$ filters without fixing the Lyapunov matrices of the $\mathcal{H}_{2}$ and $\mathcal{H}_{\infty}$ conditions. Further studies concerning reduced order filters are also of interest. Finally, the assumption that the system being observed must be stochastically stable is a very restricting one and requires further investigation in the literature. 


\section{STATIC OUTPUT FEEDBACK CONTROL FOR HIDDEN MJLS}

The goal of this chapter is to present a first formulation for the $\mathcal{H}_{2}$ and $\mathcal{H}_{\infty}$ static output feedback control in the context of hidden MJLS. The static output feedback control is known for its complexity, since even for the case without jumps it can be only formulated as a bilinear matrix inequality (BMI) problem, see, for instance, (PEAUCELLE; ARZELIER, 2001; MEHDI; BOUKAS; BACHELIER, 2004) and the survey (SADABADI; PEAUCELLE, 2016). That led to an increasing effort in the study of suboptimal and convex formulations such as the two steps procedure in (PEAUCELLE; ARZELIER, 2001; MEHDI; BOUKAS; BACHELIER, 2004), among others. In the MJLS context, we can mention (MORAIS et al., 2014) and (MORAIS et al., 2016a) that studied the $\mathcal{H}_{2}$ and $\mathcal{H}_{\infty}$ static output feedback control by means of the aforementioned two steps procedure, as well as (ZHANG; XIA; BOUKAS, 2010; CHE; WANG, 2010) that considered the $\mathcal{H}_{\infty}$ static control problem by imposing rank constraints in the system matrices.

We tackle this problem by means of the two steps procedure of (PEAUCELLE; ARZELIER, 2001; MEHDI; BOUKAS; BACHELIER, 2004), in which a stabilizing statefeedback controller is calculated and provided in the second step to calculate the static output feedback controller. Even though this formulation enables us to derive somewhat simple and convex design conditions, we can say that the results are limited to the choice of the initial guess $K$. That motivates us in finding alternative formulations for future work, i.e., as in (SONG et al., 2017) for the $l_{2}-l_{\infty}$ static output design in finite time in the detector approach, or (STADTMANN; COSTA, 2018) for the $\mathcal{H}_{\infty}$ static output feedback control for the detector approach in a continuous-time formulation.

This chapter is organized as follows. We first introduce the static controller structure and the goals in Section 6.1. The $\mathcal{H}_{2}, \mathcal{H}_{\infty}$, and mixed $\mathcal{H}_{2} / \mathcal{H}_{\infty}$ static output feedback control in the hidden MJLS approach are considered in Sections 6.2, 6.3, and 6.4, respectively. The cluster and robust cases are studied in Section 6.5, and our final remarks are stated in Section 6.6. The results of this chapter were developed in collaboration with the Centre de Recherche en Automatique de Nancy (CRAN) and can be found in (OLIVEIRA; COSTA; DAAFOUZ, 2018c) and (OLIVEIRA; COSTA; DAAFOUZ, 2018a). 


\subsection{Problem formulation}

On a probabilistic space $\left(\Omega, \mathfrak{F}\right.$, Prob) with filtration $\left\{\mathfrak{F}_{k}\right\}$ we consider the MJLS

$$
\mathcal{G}:\left\{\begin{array}{rlrl}
x(k+1) & =A_{\theta(k)} x(k)+B_{\theta(k)} u(k) & +J_{\theta(k)} w(k) \\
y(k) & =L_{\theta(k)} x(k) & & +H_{\theta(k)} w(k) \\
z_{2}(k) & =C_{\theta(k)}^{(2)} x(k)+D_{\theta(k)}^{(2)} u(k) & +E_{\theta(k)}^{(2)} w(k) \\
z_{\infty}(k) & =C_{\theta(k)}^{(\infty)} x(k)+D_{\theta(k)}^{(\infty)} u(k) & +E_{\theta(k)}^{(\infty)} w(k) \\
x(0) & =x_{0}, \theta_{0} \sim \mu,
\end{array}\right.
$$

where $x(k) \in \mathbb{R}^{n}$ is the state variable, $u(k) \in \mathbb{R}^{m}$ is the control input, $w(k) \in \mathbb{R}^{r}$ is the exogenous input, $y(k) \in \mathbb{R}^{p}$ is the measured output, and $z(k) \in \mathbb{R}^{q}$ is the controlled output. The goal is to design static output feedback controllers depending only on $\hat{\theta}(k)$ as follows

$$
u(k)=F_{\hat{\theta}(k)} y(k),
$$

Connecting (6.1) and (6.2) yields the following closed-loop system

$$
\mathcal{G}_{F}:\left\{\begin{aligned}
\tilde{x}(k+1) & =A_{\theta(k) \hat{\theta}(k)} \tilde{x}(k)+J_{\theta(k) \hat{\theta}(k)} w(k) \\
z_{2}(k) & =C_{\theta(k) \hat{\theta}(k)}^{(2)} \tilde{x}(k)+E_{\theta(k) \hat{\theta}(k)}^{(2)} w(k) \\
z_{\infty}(k) & =C_{\theta(k) \hat{\theta}(k)}^{(\infty)} \tilde{x}(k)+E_{\theta(k) \hat{\theta}(k)}^{(\infty)} w(k),
\end{aligned}\right.
$$

where

$$
\left[\begin{array}{c|c}
A_{i l} & J_{i l} \\
\hline C_{i l}^{(2)} & E_{i l}^{(2)} \\
\hline C_{i l}^{(\infty)} & E_{i l}^{(\infty)}
\end{array}\right] \triangleq\left[\begin{array}{c|c}
A_{i}+B_{i} F_{l} L_{i} & J_{i}+B_{i} F_{l} H_{i} \\
\hline C_{i}^{(2)}+D_{i}^{(2)} F_{l} L_{i} & E_{i}^{(2)}+D_{i}^{(2)} F_{l} H_{i} \\
\hline C_{i}^{(\infty)}+D_{i}^{(\infty)} F_{l} L_{i} & E_{i}^{(\infty)}+D_{i}^{(\infty)} F_{l} H_{i}
\end{array}\right],
$$

for $i \in \mathbb{N}, l \in \mathbb{M}_{i}$. We define the set of admissible controllers $\mathbb{F}$ as

$$
\mathbb{F} \triangleq\{F \text { as in (6.2) such that (6.3) is SS }\}
$$

Considering the similar spirit of Chapter 4 , we state the general mixed $\mathcal{H}_{2} / \mathcal{H}_{\infty}$ problem as follows

$$
\inf _{F \in \mathbb{F}}\left\{g(\gamma, \delta) \text { subject to }\left\|G_{F}\right\|_{2}<\gamma \text { and }\left\|G_{F}\right\|_{\infty}<\delta\right\}
$$


where $F \triangleq\left\{F_{l}, l \in \mathbb{M}\right\},\left\|G_{F}\right\|_{2}$ and $\left\|G_{F}\right\|_{\infty}$ are accordingly given in Definition 3.2 and Definition 3.3 and $g(\gamma, \delta)$ depends on the desired project (see Control Projects 1, 2, and 3).

\section{2 $\mathcal{H}_{2}$ static output feedback control for hidden MJLS}

Consider the following inequalities for a given $\gamma>0$,

$$
\begin{aligned}
& \sum_{i \in \mathbb{N}} \sum_{l \in \mathbb{M}_{i}} \mu_{i} \alpha_{i l} \operatorname{Tr}\left(W_{i l}\right)<\gamma^{2}, \\
& {\left[\begin{array}{cccc}
W_{i l} & \bullet & \bullet & \bullet \\
Z_{i l} J_{i} & \operatorname{Her}\left(Z_{i l}\right)-\mathcal{E}_{i}(P) & \bullet & \bullet \\
E_{i}^{(2)} & 0 & I & \bullet \\
-Y_{l} H_{i} & B_{i}^{\prime} Z_{i l}^{\prime} & D_{i}^{(2)^{\prime}} & \operatorname{Her}\left(X_{l}\right)
\end{array}\right]>0,} \\
& P_{i}>\sum_{l \in \mathbb{M}_{i}} \alpha_{i l} M_{i l}^{(2)}, \\
& {\left[\begin{array}{cccc}
M_{i l}^{(2)} & & & \\
G_{i l}^{(2)}\left(A_{i}+B_{i} K_{l}^{(2)}\right) & \operatorname{Her}\left(G_{i l}^{(2)}\right)-\mathcal{E}_{i}(P) & \bullet & \bullet \\
C_{i}^{(2)}+D_{i}^{(2)} K_{l}^{(2)} & 0 & I & \bullet \\
X_{l} K_{l}^{(2)}-Y_{l} L_{i} & B_{i}^{\prime} G_{i l}^{(2)} & D_{i}^{(2)^{\prime}} & \operatorname{Her}\left(X_{l}\right)
\end{array}\right]>0,}
\end{aligned}
$$

for all $i \in \mathbb{N}, l \in \mathbb{M}_{i}$. We define the set of variables of (7.7)-(7.10) as

$$
\xi_{2} \triangleq\left\{P_{i}, W_{i l}, M_{i l}^{(2)}, G_{i l}^{(2)}, Z_{i l}, X_{l}, Y_{l}, K_{l}^{(2)}, i \in \mathbb{N}, l \in \mathbb{M}_{i}\right\} \cup \xi_{\text {aux }}^{(2)},
$$

where $\xi_{\text {aux }}^{(2)} \triangleq \emptyset$ if $\gamma$ is not considered as a decision variable, and $\xi_{a u x}^{(2)}=\left\{\gamma_{a}\right\}, \gamma_{a}=\gamma^{2}$, otherwise. The set of all solutions of (6.7)-(6.10) is given by

$$
\Xi_{2} \triangleq\left\{\xi_{2}:(6.7)-(6.10) \text { holds }\right\}
$$

The set $\Xi_{2}$ is bilinear in the decision variables due to the products involving $X_{l}$ and $K_{l}^{(2)}$ as well as $G_{i l}^{(2)}$ and $K_{l}^{(2)}$. As we are going to see in this section, it is possible to provide for our formulation a sub-optimal approach in the spirit of the so-called two steps procedure introduced by (PEAUCELLE; ARZELIER, 2001).

Theorem 6.1. If $\xi_{2} \in \Xi_{2}$, then by setting (6.2) as $F_{l}=X_{l}^{-1} Y_{l}$ for all $l \in \mathbb{M}$, we have that $F \in \mathbb{F}$ and $\left\|\mathcal{G}_{F}\right\|_{2}<\gamma$. Besides, $K^{(2)} \in \mathbb{K}$. 
Proof. If (6.7)-(6.10) holds, we have that $\operatorname{Her}\left(X_{l}\right)>0$ which implies that $X_{l}$ is nonsingular. In this case, we set $F_{l}=X_{l}^{-1} Y_{l}$ and rewrite (6.8) as $\tilde{\Phi}_{i l}+\operatorname{Her}\left(U X_{l} V\left(T_{i l}\right)^{\prime}\right)>0$, where $V\left(T_{i l}\right)^{\prime} \triangleq\left[\begin{array}{cccc}-T_{i l} & 0 & 0 & I\end{array}\right], T_{i l} \triangleq F_{l} H_{i}$, and $U \triangleq\left[\begin{array}{llll}0 & 0 & 0 & I\end{array}\right]^{\prime}$, as well as

$$
\tilde{\Phi}_{i l} \triangleq\left[\begin{array}{cccc}
W_{i l} & \bullet & \bullet & \bullet \\
Z_{i l} J_{i l} & \operatorname{Her}\left(Z_{i l}\right)-\mathcal{E}_{i}(P) & \bullet & \bullet \\
E_{i l}^{(2)} & 0 & I & \bullet \\
0 & B_{i}^{\prime} Z_{i l}^{\prime} & D_{i}^{(2)^{\prime}} & 0
\end{array}\right] .
$$

By defining

$$
\mathcal{N}(X)^{\prime} \triangleq\left[\begin{array}{cccc}
I & 0 & 0 & X^{\prime} \\
0 & I & 0 & 0 \\
0 & 0 & I & 0
\end{array}\right]
$$

and by Lemma A.3 (see the Appendix A), we note that $V\left(T_{i l}\right)^{\prime} \mathcal{N}\left(T_{i l}\right)=0$ and $U^{\prime} \mathcal{N}(0)=$ 0 , and thus by multiplying (6.8) to the left hand side by $\mathcal{N}\left(T_{i l}\right)^{\prime}\left(\mathcal{N}(0)^{\prime}\right)$ and to the right by its transpose, we get that (6.8) holds only if

$$
\bar{\Phi}_{i l}\left(T_{i l}\right) \triangleq\left[\begin{array}{ccc}
W_{i l} & \bullet & \bullet \\
Z_{i l}\left(J_{i}+B_{i} T_{i l}\right) & \operatorname{Her}\left(Z_{i l}\right)-\mathcal{E}_{i}(P) & \bullet \\
E_{i}^{(2)}+D_{i}^{(2)} T_{i l} & 0 & I
\end{array}\right]>0,
$$

and $\bar{\Phi}_{i l}(0)>0$, for all $i \in \mathbb{N}, l \in \mathbb{M}_{i}$. We note that $J_{i}+B_{i} T_{i l}=J_{i}+B_{i} F_{l} H_{i}=J_{i l}$ and $E_{i}^{(2)}+D_{i}^{(2)} T_{i l}=E_{i}^{(2)}+B_{i} F_{l} T_{i l}=E_{i l}^{(2)}$, where $J_{i l}$ and $E_{i l}^{(2)}$ are given in (6.4), and thus we get that $\bar{\Phi}_{i l}\left(T_{i l}\right)$ leads to (3.19). Note also that $\bar{\Phi}_{i l}(0)>0$ leads to (3.19) with system matrices as in (4.4). Similarly, we can rewrite (6.10) as $\tilde{\Phi}_{i l}+\operatorname{Her}\left(U X_{l} V\left(S_{i l}\right)^{\prime}\right)>0$, where

$$
\tilde{\Phi}_{i l} \triangleq\left[\begin{array}{cccc}
M_{i l}^{(2)} & \bullet & \bullet & \bullet \\
G_{i l}^{(2)} A_{i l}\left(K^{(2)}\right) & \operatorname{Her}\left(G_{i l}^{(2)}\right)-\mathcal{E}_{i}(P) & \bullet & \bullet \\
C_{i l}^{(2)}\left(K^{(2)}\right) & 0 & I & \bullet \\
0 & B_{i}^{\prime} G_{i l}^{(2)} & D_{i}^{\prime} & 0
\end{array}\right],
$$

and $S_{i l} \triangleq F_{l} L_{i}-K_{l}^{(2)}$, as well as $A_{i l}\left(K^{(2)}\right) \triangleq A_{i}+B_{i} K_{l}^{(2)}$ and $C_{i l}^{(2)}\left(K^{(2)}\right) \triangleq C_{i}^{(2)}+D_{i}^{(2)} K_{l}^{(2)}$. We note that $V\left(S_{i l}\right)^{\prime} \mathcal{N}\left(S_{i l}\right)=0$, and thus by multiplying (6.15) to the left hand side by 
$\mathcal{N}\left(S_{i l}\right)^{\prime}\left(\mathcal{N}(0)^{\prime}\right)$ and to the right by its transpose, we get that (6.10) holds only if

$$
\bar{\Phi}_{i l}\left(S_{i l}\right) \triangleq\left[\begin{array}{ccc}
M_{i l}^{(2)} & \bullet & \bullet \\
G_{i l}^{(2)}\left(A_{i l}\left(K^{(2)}\right)+B_{i} S_{i l}\right) & \operatorname{Her}\left(G_{i l}^{(2)}\right)-\mathcal{E}_{i}(P) & \bullet \\
C_{i l}^{(2)}\left(K^{(2)}\right)+D_{i} S_{i l} & 0 & I
\end{array}\right]>0,
$$

and $\bar{\Phi}_{i l}(0)>0$, for all $i \in \mathbb{N}, l \in \mathbb{M}_{i}$. Thus we have that $A_{i l}\left(K^{(2)}\right)+B_{i} S_{i l}=A_{i}+B_{i} F_{l} L_{i}=$ $A_{i l}$, as well as $C_{i l}^{(2)}\left(K^{(2)}\right)+D_{i} S_{i l}=C_{i}^{(2)}+D_{i}^{(2)} F_{l} L_{i}=C_{i l}^{(2)}$, that are the matrices $A_{i l}$ and $C_{i l}^{(2)}$ in (6.4), and therefore (6.16) leads to (3.20). Since (6.7) and (6.9) are respectively equal to the first inequalities in (3.19) and (3.20), we get that $F \in \mathbb{F}$ and $\left\|\mathcal{G}_{F}\right\|_{2}<\gamma$. Similarly, by considering $\bar{\Phi}_{i l}(0)>0$ we retrieve (3.20) with system matrices given in (4.4) and thus $K^{(2)} \in \mathbb{K}$.

As previously discussed, the problem of finding a solution for (6.7)-(6.10) is bilinear due to the product of variables $X_{l}$ and $K_{l}^{(2)}$, as well as $G_{i l}^{(2)}$ and $K_{l}^{(2)}$. However, as shown in Theorem 6.1, and similarly to (PEAUCELLE; ARZELIER, 2001; MEHDI; BOUKAS; BACHELIER, 2004), a necessary condition for obtaining $\xi_{2} \in \Xi_{2}$ is that $K^{(2)}$ stochastically stabilizes the pair $(A, B)$. In that case, the next corollary provides a sub-optimal method for obtaining $F \in \mathbb{F}$. For that we define $\bar{\xi}_{2} \triangleq \xi_{2}-\left\{K_{l}^{(2)}\right\}$ (the set of variables of (6.7)-(6.10) without $\left.K_{l}^{(2)}\right)$, and $\bar{\Xi}_{2}\left(K^{(2)}\right) \triangleq$ $\left\{\bar{\xi}_{2}\right.$; such that the inequalities (6.7)-(6.10) hold $\}$.

Corollary 6.1. For a given $K^{(2)} \in \mathbb{K}$ such that $\bar{\Xi}_{2}\left(K^{(2)}\right) \neq \emptyset$, if $\bar{\xi}_{2} \in \bar{\Xi}_{2}\left(K^{(2)}\right)$, then by setting $F_{l}=X_{l}^{-1} Y_{l}$ for all $l \in \mathbb{M}$, we have that $F \in \mathbb{F}$ and $\left\|\mathcal{G}_{F}\right\|_{2}<\gamma$.

Corollary 6.1 suggests what is called the two steps procedure in the literature, that is, first a stochastic stabilizing state-feedback gain is calculated such that $\bar{\Xi}_{2}\left(K^{(2)}\right) \neq \emptyset$ and used as an input for solving (6.7)-(6.10), and finally obtaining the static output feedback controller $F$. Thus, we can write the $\mathcal{H}_{2}$ control as follows

$$
\inf _{\bar{\xi}_{2} \in \bar{\Xi}_{2}\left(K^{(2)}\right), K^{(2)} \in \mathbb{K}} \gamma_{a}
$$

for $\gamma_{a}=\gamma^{2}$. The procedure of Corollary 6.1 is shown in Algorithm 1. Since the solution set $\Xi_{2}\left(K^{(2)}\right)$ depends on the choice of $K^{(2)}$, this parameter will impact on (6.17) and the resulting value of $\gamma^{*}$. As for the case in which $\bar{\Xi}_{2}\left(K^{(2)}\right)=\emptyset$, a new state-feedback gain must be provided for the algorithm. 
Algorithm 1 The two steps procedure of Corollary 6.1

1: Calculate a stochastic stabilizing state-feedback gain $K^{(2)}$ such that $\bar{\Xi}_{2}\left(K^{(2)}\right) \neq \emptyset$;

2: Use $K_{l}^{(2)}$ as an input in (6.7)-(6.10) and calculate $F_{l}$ for all $l \in \mathbb{M}$ following Corollary 6.1 .

Example 6.1 $\mathcal{H}_{2}$ static output feedback control: We consider the system of Chapter 4, specifically Example 4.2. For the measurement equation, we set

$$
L_{1}=\left[\begin{array}{ll}
1 & 0
\end{array}\right], L_{2}=\left[\begin{array}{ll}
0 & 1
\end{array}\right], H_{1}=H_{2}=\left[\begin{array}{lll}
0 & 0 & 1
\end{array}\right]
$$

along with $\rho=0.7$ in (4.9), and use the state-feedback control gains shown in (4.16), obtained through the optimization problem (4.14), in (6.17) in order to get the following static output feedback controller

$$
\left[\begin{array}{l}
F_{1} \\
F_{2}
\end{array}\right]=\left[\frac{-0.4901}{-0.4548}\right],
$$

for $r_{\sigma}(\mathcal{L})=0.4181, \gamma^{*}=1.9575$, and $\left\|\mathcal{G}_{F}\right\|_{2}=1.6344$.

\section{3 $\mathcal{H}_{\infty}$ static output feedback control for hidden MJLS}

We now investigate the $\mathcal{H}_{\infty}$ static control. For that, we introduce the following inequalities,

$$
\begin{aligned}
& {\left[\begin{array}{cc}
Q_{i} & \bullet \\
0 & \delta^{2} I
\end{array}\right]>\sum_{l \in \mathbb{M}_{i}} \alpha_{i l}\left[\begin{array}{cc}
M_{i l}^{(\infty)} & \bullet \\
N_{i l}^{(\infty)} & S_{i l}^{(\infty)}
\end{array}\right],} \\
& {\left[\begin{array}{ccccc}
M_{i l}^{(\infty)} & \bullet & \bullet & \bullet & \bullet \\
N_{i l}^{(\infty)} & S_{i l}^{(\infty)} & \bullet & \bullet & \bullet \\
G_{i l}^{(\infty)}\left(A_{i}+B_{i} K_{l}^{(\infty)}\right) & G_{i l}^{(\infty)} J_{i} & \operatorname{Her}\left(G_{i l}^{(\infty)}\right)-\mathcal{E}_{i}(Q) & \bullet & \bullet \\
C_{i}^{(\infty)}+D_{i}^{(\infty)} K_{l}^{(\infty)} & E_{i}^{(\infty)} & 0 & I & \bullet \\
X_{l} K_{l}^{(\infty)}-Y_{l} L_{i} & -Y_{l} H_{i} & B_{i}^{\prime} G_{i l}^{(\infty)^{\prime}} & D_{i}^{(\infty)^{\prime}} & \operatorname{Her}\left(X_{l}\right)
\end{array}\right]>0 .}
\end{aligned}
$$

The set of variables of (6.18)-(6.19) is defined as follows,

$$
\xi_{\infty} \triangleq\left\{Q_{i}, M_{i l}^{(\infty)}, N_{i l}^{(\infty)}, S_{i l}^{(\infty)}, G_{i l}^{(\infty)}, X_{l}, Y_{l}, K_{l}^{(\infty)}, i \in \mathbb{N}, l \in \mathbb{M}_{i}\right\} \cup \xi_{\text {aux }}^{(\infty)},
$$


where $\xi_{\text {aux }}^{(\infty)}=\emptyset$ if $\delta$ is not considered as a decision variable, and $\xi_{\text {aux }}^{(\infty)}=\left\{\delta_{a}\right\}, \delta_{a}=\delta^{2}$, otherwise. The set of all solutions of (6.18)-(6.19) is represented by

$$
\Xi_{\infty} \triangleq\left\{\xi_{\infty} ; \text { inequalities (6.18)-(6.19) hold }\right\}
$$

Theorem 6.2. If $\xi_{\infty} \in \Xi_{\infty}$, then by setting $F_{l}=X_{l}^{-1} Y_{l}$ for all $l \in \mathbb{M}$, we have that $F \in \mathbb{F}$ and $\left\|\mathcal{G}_{F}\right\|_{\infty}<\delta$. Besides, $K^{(\infty)} \in \mathbb{K}$.

Proof. Consider that (6.18)-(6.19) hold. We have that $\operatorname{Her}\left(X_{l}\right)>0$ that implies that $X_{l}$ is non-singular. Thus, (6.19) can be rewritten $\tilde{\Phi}_{i l}+\operatorname{Her}\left(U X_{l} V\left(S_{i l}, T_{i l}\right)^{\prime}\right)>0$, where

$$
\tilde{\Phi}_{i l} \triangleq\left[\begin{array}{ccccc}
M_{i l}^{(\infty)} & \bullet & \bullet & \bullet & \bullet \\
N_{i l}^{(\infty)} & S_{i l}^{(\infty)} & \bullet & \bullet & \bullet \\
G_{i l}^{(\infty)} A_{i l}\left(K^{(\infty)}\right) & G_{i l}^{(\infty)} J_{i} & \operatorname{Her}\left(G_{i l}^{(\infty)}\right)-\mathcal{E}_{i}(Q) & \bullet & \bullet \\
C_{i l}^{(\infty)}\left(K^{(\infty)}\right) & E_{i}^{(\infty)} & 0 & I & \bullet \\
0 & 0 & B_{i}^{\prime} G_{i l}^{(\infty)^{\prime}} & D_{i}^{(\infty)^{\prime}} & 0
\end{array}\right],
$$

as well as $U \triangleq\left[\begin{array}{lllll}0 & 0 & 0 & 0 & I\end{array}\right]^{\prime}, V\left(S_{i l}, T_{i l}\right)^{\prime} \triangleq\left[\begin{array}{lllll}-S_{i l} & -T_{i l} & 0 & 0 & I\end{array}\right]$, for $S_{i l} \triangleq F_{l} L_{i}-$ $K_{l}^{(\infty)}$ and $T_{i l} \triangleq F_{l} H_{i}, F_{l}=X_{l}^{-1} Y_{l}$. We define

$$
\mathcal{N}(X, Y)^{\prime} \triangleq\left[\begin{array}{ccccc}
I & 0 & 0 & 0 & X^{\prime} \\
0 & I & 0 & 0 & Y^{\prime} \\
0 & 0 & I & 0 & 0 \\
0 & 0 & 0 & I & 0
\end{array}\right]
$$

and point out that $V\left(S_{i l}, T_{i l}\right)^{\prime} \mathcal{N}\left(S_{i l}, T_{i l}\right)=0$ and $U^{\prime} \mathcal{N}(0,0)=0$. By multiplying (6.20) to the left hand side $\mathcal{N}\left(S_{i l}, T_{i l}\right)^{\prime}$ and to the right hand side by $\mathcal{N}\left(S_{i l}, T_{i l}\right)$, we get that (6.19) holds only if

$$
\left[\begin{array}{cccc}
M_{i l}^{(\infty)} & \bullet & \bullet & \bullet \\
N_{i l}^{(\infty)} & S_{i l}^{(\infty)} & \bullet & \bullet \\
G_{i l}^{(\infty)}\left[A_{i l}\left(K^{(\infty)}\right)+B_{i} S_{i l}\right] & G_{i l}^{(\infty)}\left(J_{i}+B_{i} T_{i l}\right) & \operatorname{Her}\left(G_{i l}^{(\infty)}\right)-\mathcal{E}_{i}(Q) & \bullet \\
C_{i l}^{(\infty)}\left(K^{(\infty)}\right)+D_{i l}^{(\infty)} S_{i l} & E_{i}^{(\infty)}+D_{i}^{(\infty)} T_{i l} & 0 & I
\end{array}\right]>0
$$

and $\mathcal{N}(0,0)^{\prime} \tilde{\Phi}_{i l} \mathcal{N}(0,0)>0$. Considering the same reasoning as used in the proof of Theorem 6.1, we have that (6.18)-(6.21) implies (3.26) with system matrices in (6.4), thus $F \in F,\left\|\mathcal{G}_{F}\right\|_{\infty}<\delta$. Furthermore, since $\mathcal{N}(0,0)^{\prime} \tilde{\Phi}_{i l} \mathcal{N}(0,0)>0$, we have the 
bounded-real lemma inequalities with system matrices in (4.4) that implies that $K^{(\infty)} \in$ $\mathbb{K}$.

Similarly as it happened in the $\mathcal{H}_{2}$ control, the set $\Xi_{\infty}$ is bilinear in the decision variables due to the products between $G_{i l}^{(\infty)}$ and $K_{l}^{(\infty)}$, as well as $X_{l}$ and $K_{l}^{(\infty)}$. In the same spirit of Section 6.2, we can write the following convex approximation for the $\mathcal{H}_{\infty}$ control problem. For that we define $\bar{\xi}_{\infty} \triangleq \xi_{\infty}-\left\{K_{l}^{(\infty)}\right\}$ and $\bar{\Xi}_{\infty}\left(K^{(\infty)}\right) \triangleq$ $\left\{\bar{\xi}_{\infty}\right.$ such that the inequalities (6.18)-(6.19) hold $\}$.

Corollary 6.2. For a given $K^{(\infty)} \in \mathbb{K}$ such that $\bar{\Xi}_{\infty}\left(K^{(\infty)}\right) \neq \emptyset$, if $\bar{\xi}_{\infty} \in \bar{\Xi}_{\infty}\left(K^{(\infty)}\right)$, then by setting $F_{l}=X_{l}^{-1} Y_{l}$ for all $l \in \mathbb{M}$, we have that $F \in \mathbb{F}$ and $\left\|\mathcal{G}_{F}\right\|_{\infty}<\delta$.

Thus, we can write the $\mathcal{H}_{\infty}$ static output feedback control in the following manner,

$$
\inf _{\bar{\xi}_{\infty} \in \bar{\Xi}_{\infty}\left(K^{(\infty)}\right), K^{(\infty)} \in \mathbb{K}} \delta_{a}
$$

for $\delta_{a}=\delta^{2}$.

Algorithm 2 The two steps procedure of Corollary 6.2

1: Calculate a stochastic stabilizing state-feedback gain $K^{(\infty)}$ such that $\bar{\Xi}_{\infty}\left(K^{(\infty)}\right) \neq$ $\emptyset$;

2: Use $K_{l}^{(\infty)}$ as an input in (6.18)-(6.19) and calculate $F_{l}$ for all $l \in \mathbb{M}$ following Corollary 6.2.

Example 6.2 $\mathcal{H}_{\infty}$ static output feedback control: We consider the same system of Example 6.1 with the controlled output matrices of Example 4.3, and set $\rho=0.7$. By using the state-feedback gains in (4.23), obtained through the optimization problem (4.21), as an input to (6.22), we get the following static output feedback gains

$$
\left[\begin{array}{l}
F_{1} \\
F_{2}
\end{array}\right]=\left[\frac{-0.7636}{-0.6822}\right],
$$

for $r_{\sigma}(\mathcal{L})=0.3874, \delta^{*}=3.2200$, and $\left\|\mathcal{G}_{F}\right\|_{\infty}=2.7300$.

\subsection{The mixed $\mathcal{H}_{2} / \mathcal{H}_{\infty}$ static output feedback control}

Theorems 6.1 and 6.2 allow us to state a new result that presents conditions for calculating static controllers such that $F \in \mathbb{F},\left\|\mathcal{G}_{F}\right\|_{2}<\gamma$, and $\left\|\mathcal{G}_{F}\right\|_{\infty}<\delta$. For that, we 
define

$$
\bar{\xi} \triangleq \bar{\xi}_{(2)} \cup \bar{\xi}_{(\infty)}
$$

along with

$$
\begin{array}{r}
\bar{\Xi}\left(K^{(2)}, K^{(\infty)}\right) \triangleq\{\bar{\xi} \text { such that the LMls }(6.7)-(6.10) \text { and }(6.18)-(6.19) \\
\text { are jointly feasible }\} .
\end{array}
$$

Theorem 6.3. Given $K^{(2)} \in \mathbb{K}$ and $K^{(\infty)} \in \mathbb{K}$ such that $\bar{\Xi}\left(K^{(2)}, K^{(\infty)}\right) \neq \emptyset$, if $\bar{\xi} \in \bar{\Xi}\left(K^{(2)}, K^{(\infty)}\right)$, then by setting $F_{l}=X_{l}^{-1} Y_{l}$, we have that $F \in \mathbb{F},\left\|\mathcal{G}_{F}\right\|_{2}<\gamma$, and $\left\|\mathcal{G}_{F}\right\|_{\infty}<\delta$.

Proof. The result is a direct consequence of Theorems 6.1 and 6.2.

In this case, we rewrite the optimization problem (6.6) as follows:

$$
\inf _{\bar{\xi} \in \bar{\Xi}\left(K^{(2)}, K^{(\infty)}\right)} g(\gamma, \delta)
$$

Remark 6.1: The property described in Corollary 3.2 allows us to simplify problem (6.23) given that $z_{2}=z_{\infty}$. Then, by taking $C_{\theta(k)}=C_{\theta(k)}^{(\infty)}=C_{\theta(k)}^{(2)}, D_{\theta(k)}=D_{\theta(k)}^{(\infty)}=$ $D_{\theta(k)}^{(2)}$, and $E_{\theta(k)}=E_{\theta(k)}^{(\infty)}=E_{\theta(k)}^{(2)}$, as well as $P_{i}=Q_{i}$ and $K=K^{(2)}=K^{(\infty)}$, it is possible to redefine $\bar{\phi}$ as $\bar{\xi} \triangleq \bar{\xi}_{\infty} \cup\left\{W_{i l}, Z_{i l}, i \in \mathbb{N}, l \in \mathbb{M}_{i}\right\}$ and $\bar{\Xi} \triangleq\{\bar{\xi}$ such that (6.7) (6.8) and (6.18) - (6.19) jointly hold $\}$ in (6.23) and Theorem 6.3 would still hold.

Remark 6.2: In the similar spirit of (MORAIS et al., 2016a) and (MORAIS et al., $2016 b)$, it is possible to design dynamic output feedback full or reduced order controllers through (6.7)-(6.10) and (6.18)-(6.19). In this case, note that by controlling (6.1) with the following structure

$$
\mathcal{C}:\left\{\begin{aligned}
x_{c}(k+1) & =A_{c \hat{\theta}(k)} x_{c}(k)+B_{c \hat{\theta}(k)} y(k) \\
u(k) & =C_{c \hat{\theta}(k)} x_{c}(k)+D_{c \hat{\theta}(k)} y(k),
\end{aligned}\right.
$$

where $x_{c}(k) \in \mathbb{R}^{n_{c}}, 0 \leq n_{c} \leq n$, it is possible to rewrite the respective closed-loop 
system as follows

$$
\begin{aligned}
& \begin{aligned}
\tilde{x}(k+1) & =\left[\begin{array}{cc}
A_{\theta(k)} & 0 \\
0 & 0_{n_{c}}
\end{array}\right] \tilde{x}(k)+\left[\begin{array}{cc}
0 & B_{\theta(k)} \\
I_{n_{c}} & 0
\end{array}\right] \tilde{u}(k)+\left[\begin{array}{c}
J_{\theta(k)} \\
0
\end{array}\right] w(k), \\
\tilde{y}(k) & =\left[\begin{array}{cc}
0 & I_{n_{c}} \\
L_{\theta(k)} & 0
\end{array}\right] \tilde{x}(k)
\end{aligned} \\
& z_{2}(k)=\left[\begin{array}{ll}
C_{\theta(k)}^{(2)} & 0
\end{array}\right] \tilde{x}(k)+\left[\begin{array}{ll}
0 & D_{\theta(k)}^{(2)}
\end{array}\right] \tilde{u}(k)+\left[E_{\theta(k)}^{(2)}\right] w(k), \\
& z_{\infty}(k)=\left[\begin{array}{ll}
C_{\theta(k)}^{(\infty)} & 0
\end{array}\right] \tilde{x}(k)+\left[\begin{array}{ll}
0 & D_{\theta(k)}^{(\infty)}
\end{array}\right] \tilde{u}(k)+\left[E_{\theta(k)}^{(\infty)}\right] w(k),
\end{aligned}
$$

where $\tilde{x}(k)=\left[\begin{array}{ll}x(k)^{\prime} & x_{c}(k)^{\prime}\end{array}\right]^{\prime}$ and

$$
\tilde{u}(k)=\left[\begin{array}{ll}
A_{c \hat{\theta}(k)} & B_{c \hat{\theta}(k)} \\
C_{c \hat{\theta}(k)} & D_{c \hat{\theta}(k)}
\end{array}\right] \tilde{y}(k),
$$

that is, the problem of finding a dynamic output feedback controller is converted into finding a static one. Then, an alternative to the results presented in Chapter 7 is to use (6.7)-(6.10) and (6.18)-(6.19) with the modified system matrices in (6.24).

Example 6.3: For $\rho=0.7$ we use (4.16) and (4.23) as inputs to (6.23). By selecting the upper bound $\delta=16$ and minimizing $\gamma$ in (6.23), the resulting mixed $\mathcal{H}_{2} / \mathcal{H}_{\infty}$ dynamic controller is given below

$$
\left[\frac{F_{1}}{F_{2}}\right]=\left[\frac{-0.5203}{-0.4913}\right],
$$

with $r_{\sigma}(\mathcal{L})=0.4065, \gamma_{F}^{*}=1.9808,\left\|\mathcal{G}_{F}\right\|_{2}=1.6494, \delta_{F}=4$, and $\left\|\mathcal{G}_{F}\right\|_{\infty}=2.6306$.

\subsection{Static output feedback control for the cluster case and robust control}

Considering the properties of our formulation, it is possible to obtain cluster and consequently mode-independent static output feedback controllers through problems (6.17), (6.22), and (6.23). Our conditions retrieve a modified version of the ones in (MORAIS et al., 2016a) and (MORAIS et al., 2014). 
Consider the following inequalities,

$$
\begin{aligned}
& \sum_{i \in \mathbb{N}} \operatorname{Tr}\left(\bar{W}_{i}\right)<\gamma^{2}, \\
& {\left[\begin{array}{cccc}
\bar{W}_{i} & \bullet & \bullet & \bullet \\
Z_{i} J_{i} & \operatorname{Her}\left(Z_{i}\right)-\mathcal{E}_{i}(P) & \bullet & \bullet \\
E_{i}^{(2)} & 0 & I & \bullet \\
-Y^{s} H_{i} & B_{i}^{\prime} Z_{i}^{\prime} & D_{i}^{(2)^{\prime}} & \operatorname{Her}\left(X^{s}\right)
\end{array}\right]>0,}
\end{aligned}
$$

$\left[\begin{array}{cccc}P_{i} & \bullet & \bullet & \bullet \\ G_{i}^{(2)}\left(A_{i}+B_{i} K^{(2) s}\right) & \operatorname{Her}\left(G_{i}^{(2)}\right)-\mathcal{E}_{i}(P) & \bullet & \bullet \\ C_{i}^{(2)}+D_{i}^{(2)} K^{(2) s} & 0 & I & \bullet \\ X^{s} K^{(2) s}-Y^{s} L_{i} & B_{i}^{\prime} G_{i}^{(2)^{\prime}} & D_{i}^{(2)^{\prime}} & \operatorname{Her}\left(X^{s}\right)\end{array}\right]>0$,

along with

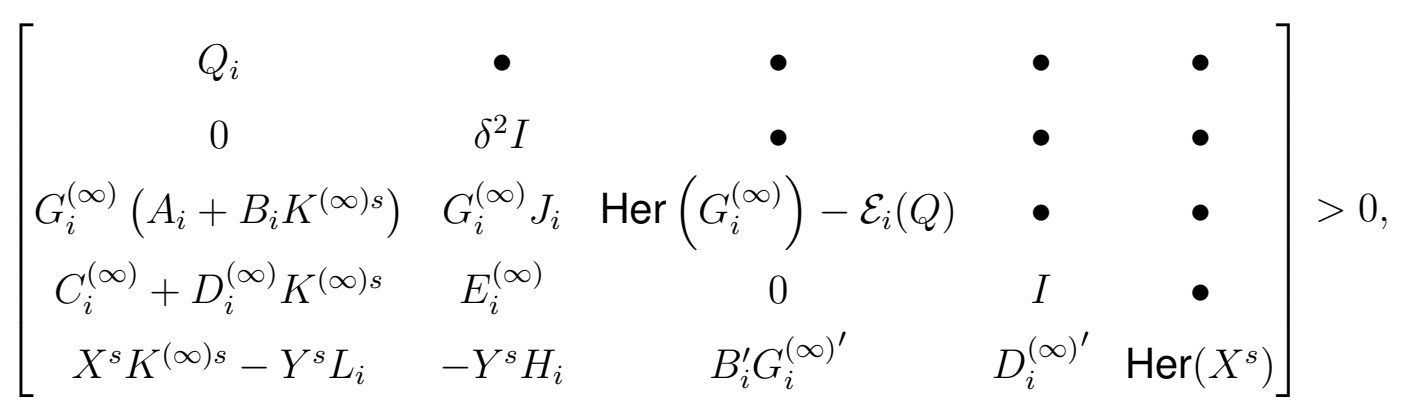

for $s \in\{1, \ldots, \kappa\}, i \in \mathbb{N}^{s}$.

Proposition 6.1: Under Assumption 3.1, we have that

- For a given $K_{l}^{(2)}=K^{(2) s}, l \in \mathbb{M}^{s}, s \in\{1, \ldots, \kappa\}$, Equations (6.7)-(6.10) and (6.25)-(6.27) are equivalent;

- For a given $K_{l}^{(\infty)}=K^{(\infty) s}, l \in \mathbb{M}^{s}, s \in\{1, \ldots, \kappa\}$, Equations (6.18)-(6.19) and (6.28) are equivalent.

Proof. It follows the similar reasoning employed in the proof of Proposition 4.1.

In a similar spirit as in (MORAIS et al., 2016a) and (MORAIS et al., 2014), since the parametrization of $F$ does not involve the transition and conditional probabilities, and besides (6.7)-(6.10) and (6.18)-(6.19) are affine with respect to $p_{i j}$ and $\alpha_{i l}$, we assume that $\mathbb{P} \in \mathbb{D}_{\mathbb{P}}$ and $\Upsilon \in \mathbb{D}_{\Upsilon}$. We define the set $\mathbb{F}_{r} \triangleq\left\{F:(6.3)\right.$ for $\mathbb{P} \in \mathbb{D}_{\mathbb{P}}$ and $\Upsilon \in$ 
$\mathbb{D}_{\Upsilon}$ is $\left.S S\right\}$, and also set

$$
p_{i j}=p_{i j}^{(h)}, \alpha_{i l}=\alpha_{i l}^{(t)}
$$

in (6.7)-(6.10) and (6.18)-(6.19) for $s \in\{1, \ldots, \eta\}, t \in\{1, \ldots, \tau\}, i \in \mathbb{N}, l \in \mathbb{M}_{i}$. We define $\tilde{\Xi}\left(K^{(2)}, K^{(\infty)}\right)$ in this case by considering the changes described in (6.29)

$$
\tilde{\Xi}\left(K^{(2)}, K^{(\infty)}\right)=\{\bar{\xi} \text { such that the LMIs }(6.7)-(6.10) \text { and }(6.18)-(6.19) \text { subject to }
$$

(6.29) are jointly feasible\}.

Corollary 6.3. Given $K^{(2)} \in \mathbb{K}_{r}$ and $K^{(\infty)} \in \mathbb{K}_{r}$ such that $\tilde{\Xi}\left(K^{(2)}, K^{(\infty)}\right) \neq \emptyset$, if $\bar{\xi} \in$ $\tilde{\Xi}\left(K^{(2)}, K^{(\infty)}\right)$, then by setting $F_{l}=X_{l}^{-1} Y_{l}$ for all $l \in \mathbb{M}$, we get that $F \in \mathbb{F}_{r},\left\|\mathcal{G}_{F}\right\|_{2}<\gamma$ and $\left\|\mathcal{G}_{F}\right\|_{\infty}<\delta$.

Example 6.4: Recalling Assumption 3.1, we set $\rho=0.5$ in (4.9) and calculate the $\mathcal{H}_{2}$ state-feedback controller through (4.14), in order to get

$$
K_{i}=\left[\begin{array}{ll}
-0.5895 & -0.0266
\end{array}\right]
$$

for all $i \in \mathbb{N}$, with $\gamma_{K}^{*}=1.2422$. Then we use (6.30) in (6.17) in order to get the following $\mathcal{H}_{2}$ static output feedback controller

$$
F_{i}=-0.4805
$$

for all $i \in \mathbb{N}$, with $r_{\sigma}(\mathcal{L})=0.4176, \gamma_{F}^{*}=1.9735$, and $\left\|\mathcal{G}_{F}\right\|_{2}=1.6344$.

On the other hand, for the $\mathcal{H}_{\infty}$ control, we design a $\mathcal{H}_{\infty}$ state-feedback controller through (4.21) with $\rho=0.5$ in order to get

$$
K_{i}=\left[\begin{array}{ll}
-0.4857 & -0.1532
\end{array}\right]
$$

for all $i \in \mathbb{N}$, with $\delta=7.2054$ and $\delta_{K}=2.6980$. By applying $K_{l}$ in (6.22), we calculate the following $\mathcal{H}_{\infty}$ controller

$$
F_{i}=-0.7451
$$

for all $i \in \mathbb{N}$, with $r_{\sigma}(\mathcal{L})=0.3874, \delta=3.2846$, and $\delta_{K}=3.0143$. 


\subsection{Final remarks}

In this chapter, we studied the design of static output feedback controllers in the context of partial observation of $\hat{\theta}(k)$, for the $\mathcal{H}_{2}, \mathcal{H}_{\infty}$, and mixed $\mathcal{H}_{2} / \mathcal{H}_{\infty}$ control. Bilinear synthesis conditions were given that can formulated in a convex two steps procedure inspired in (PEAUCELLE; ARZELIER, 2001; MEHDI; BOUKAS; BACHELIER, 2004) and (MORAIS et al., 2014; MORAIS et al., 2016a) for MJLS. We stress that the proposed solution is suboptimal and depends on the choice of the state-feedback gain, and thus some alternative formulations are also desirable, such as the ones given in (STADTMANN; COSTA, 2018) 


\section{DYNAMIC OUTPUT FEEDBACK CONTROL FOR HIDDEN MJLS}

In this chapter we study the $\mathcal{H}_{2}$ and $\mathcal{H}_{\infty}$ dynamic output feedback control in the context of hidden MJLS. This is a very challenging problem since there is no result in the literature that presents conditions for the parametrization of the dynamic controller structure in this case. It is also noteworthy that there are few results dealing with the cluster and mode-independent cases due to its complexity. In this chapter, we directly tackle this problem by first providing bilinear matrix inequality conditions for the design of full-order controllers depending only on $\hat{\theta}(k)$ such that the $\mathcal{H}_{2}$ and/or $\mathcal{H}_{\infty}$ norms of the closed-loop system are bounded. Given this formulation, we show that a necessary condition for the feasibility of our BMls is that the pair $(A, B)$ is stochastically stabilizable, that enables us to present a type of ad hoc separation procedure in the spirit of (OLIVEIRA, 1999) and (OLIVEIRA; GEROMEL; BERNUSSOU, 2000). First a stabilizing state-feedback controller is calculated and used as input in order to derive the remaining $\hat{\theta}(k)$-dependent controller matrices. Additionally, our conditions becomes also necessary in the case of complete observation of the Markov chain, that is, $\hat{\theta}=\theta$, and thus we are able to retrieve the result in (GEROMEL; GONÇALVES; FIORAVANTI, 2009) for strictly proper controller structures by using suitable choices of state-feedback controllers as inputs.

Recalling the discussion in Chapter 2, considering a perfect observation of $\theta(k)$, the aforementioned work (COSTA; TUESTA, 2004) tackled the design of $\mathcal{H}_{2}$ dynamic controllers through the lens of the celebrated separation procedure that echoes the classic LQG control, and thus the final structure is composed by the optimal LQR state-feedback controller and the optimal Luenberger gain of the $\mathcal{H}_{2}$ filter, both obtained by means of coupled algebraic Riccati equations (CARE). Conversely the work (GEROMEL; GONÇALVES; FIORAVANTI, 2009) investigated the design of proper dynamic controllers in the context of $\mathcal{H}_{2}$ and $\mathcal{H}_{\infty}$ control, where necessary and sufficient conditions are given in the LMI formulation. As for the cluster and mode-independent cases, we may cite a few works such as (FIORAVANTI; GONÇALVES; GEROMEL, 2013a) that presents model-based controllers, and due to this structural choice, the system matrices must be equal inside a given cluster for providing cluster-dependent 
controllers. By writing the dynamic design problem as one of static controllers, the works (MORAIS et al., 2016b) and (MORAIS et al., 2016a) presented sub-optimal design conditions in the two-steps procedure of (PEAUCELLE; ARZELIER, 2001) and (MEHDI; BOUKAS; BACHELIER, 2004). More recently the work (MORAIS et al., 2017) presented sub-optimal conditions for obtaining cluster and mode-independent controller by means of an iterative algorithm. Note that the strategy presented in this thesis applied to the context of partial observation case is new due to the difficulties that arise in the convexification of the problem. In this sense our work (OLIVEIRA; COSTA; DAAFOUZ, 2018b) ${ }^{1}$ proposed the ad hoc separation procedure strategy applied for the hidden MJLS, that can also be used in the cluster and mode-independent cases.

The structure of this chapter is as follows. Section 7.1 introduces the problem formulation, Section 7.2 presents design conditions for the $\mathcal{H}_{2}$ dynamic output feedback control. In Section 7.3, the $\mathcal{H}_{\infty}$ dynamic output feedback control problem is studied and given the conditions in Sections 7.2 and 7.3, we present the mixed $\mathcal{H}_{2} / \mathcal{H}_{\infty}$ control design conditions in Section 7.4. The cluster and mode-independent cases are studied in Section 7.5, along with the dynamic output feedback robust control with respect to the transitions and conditional probabilities. Our final remarks are presented in Section 7.6.

\subsection{Problem formulation}

On a probabilistic space $\left(\Omega, \mathfrak{F}\right.$, Prob) with filtration $\left\{\mathcal{F}_{k}\right\}$ we consider the MJLS

$$
\mathcal{G}:\left\{\begin{array}{rlrl}
x(k+1) & =A_{\theta(k)} x(k)+B_{\theta(k)} u(k) & +J_{\theta(k)} w(k) \\
y(k) & =L_{\theta(k)} x(k) & & +H_{\theta(k)} w(k) \\
z_{2}(k) & =C_{\theta(k)}^{(2)} x(k)+D_{\theta(k)}^{(2)} u(k) & +E_{\theta(k)}^{(2)} w(k) \\
z_{\infty}(k) & =C_{\theta(k)}^{(\infty)} x(k)+D_{\theta(k)}^{(\infty)} u(k) & +E_{\theta(k)}^{(\infty)} w(k) \\
x(0) & =x_{0}, \theta_{0} \sim \mu,
\end{array}\right.
$$

where $x(k) \in \mathbb{R}^{n}$ is the state variable, $u(k) \in \mathbb{R}^{m}$ is the control input, $w(k) \in \mathbb{R}^{r}$ is the exogenous input, $y(k) \in \mathbb{R}^{p}$ is the measured output, and $z_{2}(k) \in \mathbb{R}^{q_{2}}$ and $z_{\infty}(k) \in \mathbb{R}^{q_{\infty}}$ are the controlled output. We want to design strictly proper controllers with the following

\footnotetext{
${ }^{1}$ Written in collaboration with the Centre de Recherche en Automatique de Nancy (CRAN).
} 
structure,

$$
\mathcal{C}:\left\{\begin{aligned}
x_{c}(k+1) & =A_{c \hat{\theta}(k)} x_{c}(k)+B_{c \hat{\theta}(k)} y(k) \\
u(k) & =C_{c \hat{\theta}(k)} x_{c}(k) \\
x_{c}(0) & =x_{c 0},
\end{aligned}\right.
$$

where $x_{c}(k) \in \mathbb{R}^{n}$, that is, a full-order controller. Connecting (7.1) and (7.2) yields the following closed-loop system

$$
\mathcal{G}_{c}:\left\{\begin{aligned}
\tilde{x}(k+1) & =A_{\theta(k) \hat{\theta}(k)} \tilde{x}(k)+J_{\theta(k) \hat{\theta}(k)} w(k) \\
z_{2}(k) & =C_{\theta(k) \hat{\theta}(k)}^{(2)} \tilde{x}(k)+E_{\theta(k) \hat{\theta}(k)}^{(2)} w(k) \\
z_{\infty}(k) & =C_{\theta(k) \hat{\theta}(k)}^{(\infty)} \tilde{x}(k)+E_{\theta(k) \hat{\theta}(k)}^{(\infty)} w(k),
\end{aligned}\right.
$$

where $\tilde{x}(k) \in \mathbb{R}^{2 n}$ is given by $\tilde{x}(k)^{\prime}=\left[\begin{array}{ll}x(k)^{\prime} & x_{c}(k)^{\prime}\end{array}\right]$, and

$$
\left[\begin{array}{c|c}
A_{i l} & J_{i l} \\
\hline C_{i l}^{(2)} & E_{i l}^{(2)} \\
\hline C_{i l}^{(\infty)} & E_{i l}^{(\infty)}
\end{array}\right] \triangleq\left[\begin{array}{cc|c}
A_{i} & B_{i} C_{c l} & J_{i} \\
B_{c l} L_{i} & A_{c l} & B_{c l} H_{i} \\
\hline C_{i}^{(2)} & D_{i}^{(2)} C_{c l} & E_{i}^{(2)} \\
\hline C_{i}^{(\infty)} & D_{i}^{(\infty)} C_{c l} & E_{i}^{(\infty)}
\end{array}\right],
$$

for $i \in \mathbb{N}, l \in \mathbb{M}_{i}$. We define the set of admissible controllers $\mathbb{C}$ as

$$
\mathbb{C}=\{\mathcal{C} \text { as in (7.2) such that (7.3) is SS }\}
$$

Similarly as we did in Chapters 4 and 5 , we state a general mixed $\mathcal{H}_{2} / \mathcal{H}_{\infty}$ problem as follows

$$
\inf _{\mathcal{C} \in \mathbb{C}}\left\{g(\gamma, \delta) \text { subject to }\left\|G_{c}\right\|_{2}<\gamma \text { and }\left\|G_{c}\right\|_{\infty}<\delta\right\},
$$

where $\left\|G_{c}\right\|_{2}$ and $\left\|G_{c}\right\|_{\infty}$ are accordingly given in Definition 3.2 and Definition 3.3 and $g(\gamma, \delta)$ depends on the desired project, similarly as in Chapter 4 (see Control Projects 1, 2, and 3).

Using the system matrices (7.4) in (3.19) and (3.20), and trying to minimize $\gamma^{2}$ with respect to $\left(A_{c l}, B_{c l}, C_{c l}\right)$ and $\left(W_{i l}, M_{i l}^{(2)}, P_{i}\right)$ is not an option since the feasibility set of the resulting problem is highly non-linear (the same thing can be said with respect to the $\mathcal{H}_{\infty}$ control problem). Another option would be trying to directly use the congruence transformations of (GEROMEL; GONÇALVES; FIORAVANTI, 2009), however the one-to-one change of variables depends also on the system matrices. In this case, the 
resulting controller matrices would depend not only on $\theta(k)$ but also on $\hat{\theta}(k)$ (a similar effect can be noted in the work (ABERKANE; SAUTER; PONSART, 2008)). Instead, we aim at finding a BMl formulation akin to the ones given in (GEROMEL; BERNUSSOU; OLIVEIRA, 1999), (OLIVEIRA, 1999), and (OLIVEIRA; GEROMEL; BERNUSSOU, 2000), in the context of LTI systems and decentralized control. For that, we used the ideas in (GEROMEL; GONÇALVES; FIORAVANTI, 2009) and (GONÇALVES; FIORAVANTI; GEROMEL, 2010) applied in a different way in order to get our formulation.

\section{2 $\mathcal{H}_{2}$ dynamic output feedback control for hidden MJLS}

Consider the following inequalities,

$$
\begin{aligned}
& \sum_{i \in \mathbb{N}} \sum_{l \in \mathbb{M}_{i}} \mu_{i} \alpha_{i l} \operatorname{Tr}\left(W_{i l}\right)<\gamma^{2}, \\
& {\left[\begin{array}{cccc}
W_{i l} & \bullet & \bullet & \bullet \\
\mathcal{E}_{i}(Y) J_{i} & \mathcal{E}_{i}(Y) & \bullet & \bullet \\
G_{l} J_{i}+F_{l} H_{i} & 0 & \operatorname{Her}\left(G_{l}\right)+\mathcal{E}_{i}(Y)-\mathcal{E}_{i}(X) & \bullet \\
E_{i}^{(2)} & 0 & 0 & I
\end{array}\right]>0,} \\
& {\left[\begin{array}{cc}
Y_{i} & \bullet \\
Y_{i} & X_{i}
\end{array}\right]>\sum_{l \in \mathbb{M}_{i}} \alpha_{i l}\left[\begin{array}{cc}
M_{i l}^{(2,1)} & \bullet \\
M_{i l}^{(2,2)} & M_{i l}^{(2,3)}
\end{array}\right] \text {, }} \\
& {\left[\begin{array}{ccccc}
M_{i l}^{(2,1)} & \bullet & \bullet & \bullet & \bullet \\
M_{i l}^{(2,2)} & M_{i l}^{(2,3)} & \bullet & \bullet & \bullet \\
\mathcal{E}_{i}(Y)\left(A_{i}+B_{i} K_{l}\right) & \mathcal{E}_{i}(Y) A_{i} & \mathcal{E}_{i}(Y) & \bullet & \bullet \\
G_{l}\left(A_{i}+B_{i} K_{l}\right)+F_{l} L_{i}+R_{l} & G_{l} A_{i}+F_{l} L_{i} & 0 & \operatorname{Her}\left(G_{l}\right)+\mathcal{E}_{i}(Y)-\mathcal{E}_{i}(X) & \bullet \\
C_{i}^{(2)}+D_{i}^{(2)} K_{l} & C_{i}^{(2)} & 0 & 0 & I
\end{array}\right]>0,}
\end{aligned}
$$

for all $i \in \mathbb{N}, l \in \mathbb{M}_{i}$. We define the set of variables of (7.7)-(7.10) as

$$
\xi_{2} \triangleq\left\{M_{i l}^{(2,1)}, M_{i l}^{(2,2)}, M_{i l}^{(2,3)}, Y_{i}, X_{i}, G_{l}, K_{l}, F_{l}, R_{l}, i \in \mathbb{N}, l \in \mathbb{M}_{i}\right\} \cup \xi_{\text {aux }}^{(2)}
$$

where $\xi_{\text {aux }}^{(2)}=\emptyset$ if $\gamma$ is given, or $\xi_{\text {aux }}^{(2)}=\left\{\gamma_{a}\right\}, \gamma_{a} \triangleq \gamma^{2}$ if $\gamma$ is a problem variable. The set of all solutions of (7.7)-(7.10) is given by

$$
\Xi_{2} \triangleq\left\{\xi_{2}:(7.7)-(7.10) \text { holds }\right\}
$$


The feasibility set $\Xi_{2}$ is bilinear in the decision variables, where the source of nonlinearity arises due to the products involving $\mathcal{E}_{i}(Y)$ and $K_{l}$, as well as $G_{l}$ and $K_{l}$. As we are going to see in this section, we can provide a convex formulation by conveniently holding $K_{l}$ fixed following a classical assumption.

Theorem 7.1. Consider the following statements:

(i) There exists $\xi_{2}$ such that $\xi_{2} \in \Xi_{2}$.

(ii) There exists a controller $\mathcal{C}$ such that $\mathcal{C} \in \mathbb{C}$ and $\left\|\mathcal{G}_{c}\right\|_{2}<\gamma$.

We have that $(i) \Longrightarrow($ ii $)$ by setting $A_{c l}=-G_{l}^{-1} R_{l}, B_{c l}=-G_{l}^{-1} F_{l}$, and $C_{c l}=K_{l}$ for all $l \in \mathbb{M}$. Moreover $(i i) \Longrightarrow($ i) whenever $\hat{\theta}=\theta$.

Proof. $(i) \Longrightarrow$ (ii): Considering that (7.7)-(7.10) hold, then by the left-hand side of (7.9), we note that $X_{i}-Y_{i}>0$, and so by (7.10), we have that $\operatorname{Her}\left(G_{l}\right)>\mathcal{E}_{i}(X-Y)>0$ for all $i \in \mathbb{N}, l \in \mathbb{M}_{i}$, which implies that $G_{l}$ is non-singular. Setting $R_{l}=-G_{l} A_{c l}$ and $F_{l}=-G_{l} B_{c l}$ and bearing in mind Lemma A.2 allows us to rewrite (7.8) and (7.9) as follows

$$
\begin{gathered}
{\left[\begin{array}{cccc}
W_{i l} & \bullet & \bullet & \bullet \\
\mathcal{E}_{i}(Y) J_{i} & \mathcal{E}_{i}(Y) & \bullet & \bullet \\
G_{l}\left(J_{i}-B_{c l} H_{i}\right) & 0 & \mathcal{X}_{i l}^{(1)} & \bullet \\
E_{i}^{(2)} & 0 & 0 & I
\end{array}\right]>0,} \\
{\left[\begin{array}{ccccc}
M_{i l}^{(2,1)} & & & & \\
M_{i l}^{(2,2)} & M_{i l}^{(2,3)} & \bullet & \bullet & \bullet \\
\mathcal{E}_{i}(Y)\left(A_{i}+B_{i} C_{c l}\right) & \mathcal{E}_{i}(Y) A_{i} & \mathcal{E}_{i}(Y) & \bullet & \bullet \\
G_{l}\left(A_{i}+B_{i} C_{c l}\right)-G_{l}\left(A_{c l}+B_{c l} L_{i}\right) & G_{l}\left(A_{i}-B_{c l} L_{i}\right) & 0 & \mathcal{X}_{i l}^{(1)} & \bullet \\
C_{i}^{(2)}+D_{i}^{(2)} C_{c l} & C_{i}^{(2)} & 0 & 0 & I
\end{array}\right]>0,}
\end{gathered}
$$

also hold, where $\mathcal{X}_{i l}^{(1)} \triangleq G_{l}\left[\mathcal{E}_{i}(X)-\mathcal{E}_{i}(Y)\right]^{-1} G_{l}^{\prime}$. Inspired in the work (GONÇALVES; FIORAVANTI; GEROMEL, 2010), which is a MJLS filtering work, and similarly as we did in Chapter 5 , we define the matrix

$$
\mathcal{D}_{i l}=\left[\begin{array}{cc}
I & I \\
0 & G_{l}^{-T}\left[\mathcal{E}_{i}(X)-\mathcal{E}_{i}(Y)\right]
\end{array}\right]
$$


and apply the congruence transformations $\operatorname{diag}\left(I_{r}, \mathcal{D}_{i l}, I_{q}\right)$ and $\operatorname{diag}\left(I_{2 n}, \mathcal{D}_{i l}, I_{q}\right)$ to (7.13) and (7.14) respectively to obtain that

$$
\begin{aligned}
& {\left[\begin{array}{cccc}
W_{i l} & \bullet & \bullet & \bullet \\
\mathcal{E}_{i}(Y) J_{i} & \mathcal{E}_{i}(Y) & \bullet & \bullet \\
\mathcal{E}_{i}(X) J_{l}+\mathcal{E}_{i}(Y-X) B_{c l} H_{i} & \mathcal{E}_{i}(Y) & \mathcal{E}_{i}(X) & \bullet \\
E_{i}^{(2)} & 0 & 0 & I
\end{array}\right]>0,} \\
& {\left[\begin{array}{ccccc}
M_{i l}^{(2,1)} & \bullet & \bullet & \bullet & \bullet \\
M_{i l}^{(2,2)} & M_{i l}^{(2,3)} & \bullet & \bullet & \bullet \\
\mathcal{E}_{i}(Y)\left(A_{i}+B_{i} C_{c l}\right) & \mathcal{E}_{i}(Y) A_{i} & \mathcal{E}_{i}(Y) & \bullet & \bullet \\
\mathcal{X}_{i l}^{(2)} & \mathcal{E}_{i}(X) A_{i}+\mathcal{E}_{i}(Y-X) B_{c l} L_{i} & \mathcal{E}_{i}(Y) & \mathcal{E}_{i}(X) & \bullet \\
C_{i}^{(2)}+D_{i}^{(2)} C_{c l} & C_{i}^{(2)} & 0 & 0 & I
\end{array}\right]>0,}
\end{aligned}
$$

hold, where $\mathcal{X}_{i l}^{(2)} \triangleq \mathcal{E}_{i}(X)\left(A_{i}+B_{i} C_{c l}\right)+\mathcal{E}_{i}(Y-X)\left(A_{c l}+B_{c l} L_{i}\right)$. Applying the congruence transformations $\operatorname{diag}\left(I_{r}, \mathcal{E}_{i}(Y)^{-1}, I_{n}, I_{q_{2}}\right)$ and $\operatorname{diag}\left(Y_{i}^{-1}, \mathcal{E}_{i}(Y)^{-1}, I_{n}, I_{q_{2}}\right)$ to the previous inequalities, as well as $\operatorname{diag}\left(Y_{i}^{-1}, I_{n}\right)$ to $(7.9)$ yields to

$$
\begin{aligned}
& {\left[\begin{array}{cc}
Y_{i}^{-1} & \bullet \\
I & X_{i}
\end{array}\right]>\sum_{l \in \mathbb{M}_{i}} \alpha_{i l}\left[\begin{array}{cc}
\bar{M}_{i l}^{(2,1)} & \bullet \\
\bar{M}_{i l}^{(2,2)} & M_{i l}^{(2,3)}
\end{array}\right] \text {, }} \\
& {\left[\begin{array}{cccc}
W_{i l} & \bullet & \bullet & \bullet \\
J_{i} & \mathcal{E}_{i}(Y)^{-1} & \bullet & \bullet \\
\mathcal{E}_{i}(X) J_{l}+\mathcal{E}_{i}(Y-X) B_{c l} H_{i} & I & \mathcal{E}_{i}(X) & \bullet \\
E_{i}^{(2)} & 0 & 0 & I
\end{array}\right]>0 \text {, }} \\
& {\left[\begin{array}{ccccc}
\bar{M}_{i l}^{(2,1)} & \bullet & \bullet & \bullet & \bullet \\
\bar{M}_{i l}^{(2,2)} & M_{i l}^{(2,3)} & \bullet & \bullet & \bullet \\
\left(A_{i}+B_{i} C_{c l}\right) Y_{i}^{-1} & A_{i} & \mathcal{E}_{i}(Y)^{-1} & \bullet & \bullet \\
\mathcal{X}_{i l}^{(2)} Y_{i}^{-1} & \mathcal{E}_{i}(X) A_{i}+\mathcal{E}_{i}(Y-X) B_{c l} L_{i} & I & \mathcal{E}_{i}(X) & \bullet \\
\left(C_{i}^{(2)}+D_{i}^{(2)} C_{c l}\right) Y_{i}^{-1} & C_{i}^{(2)} & 0 & 0 & I
\end{array}\right]>0 .}
\end{aligned}
$$

Then, by imposing the structures

$$
P_{i}=\left[\begin{array}{cc}
X_{i} & Y_{i}-X_{i} \\
Y_{i}-X_{i} & X_{i}-Y_{i}
\end{array}\right]
$$


and

$$
M_{i l}^{(2)}=\left[\begin{array}{cc}
M_{i l}^{(2,3)} & \bullet \\
M_{i l}^{(2,2)^{\prime}}-M_{i l}^{(2,3)} & M_{i l}^{(1,1)}-\operatorname{Her}\left(M_{i l}^{2,2}\right)+M_{i l}^{(2,3)}
\end{array}\right]
$$

in (3.19)-(3.20), we get that $\hat{U}_{i}=-\hat{X}_{i}=Y_{i}-X_{i}$ in (A.1) and recalling the discussion in Appendix $\mathrm{A}$, we have that $\hat{V}_{i}=\hat{V}_{i}^{\prime}=Y_{i}^{-1}$. Besides, by bearing in mind the definitions of $\mathcal{T}_{i}^{(2)}$ and $\mathcal{H}_{i}^{(2)}$ in (A.7) and (A.8)

$$
\mathcal{T}_{i}^{(2)}=\left[\begin{array}{cc}
Y_{i}^{-1} & I \\
\hat{V}_{i}^{\prime} & 0
\end{array}\right] \text { such that } \mathcal{T}_{i}^{(2)^{\prime}} P_{i} \mathcal{T}_{i}^{(2)}=\left[\begin{array}{cc}
Y_{i}^{-1} & I \\
I & X_{i}
\end{array}\right]
$$

as well as

$$
\mathcal{H}_{i}^{(2)}=\left[\begin{array}{ll}
I & \mathcal{E}_{i}(X) \\
0 & \mathcal{E}_{i}(\hat{U})^{\prime}
\end{array}\right] \text { such that } \mathcal{H}_{i}^{(2)^{\prime}} \mathcal{E}_{i}(P)^{-1} \mathcal{H}_{i}^{(2)}=\left[\begin{array}{cc}
\hat{P}_{1 i} & I \\
I & \mathcal{E}_{i}(X)
\end{array}\right],
$$

with $\hat{U}_{i}=Y_{i}-X_{i}$ and $\hat{P}_{1 i}=\left[\mathcal{E}_{i}(X)-\mathcal{E}_{i}(\hat{U}) \mathcal{E}_{i}(\hat{X})^{-1} \mathcal{E}_{i}(\hat{U})^{\prime}\right]^{-1}=\mathcal{E}_{i}(X+\hat{U})^{-1}=\mathcal{E}_{i}(Y)^{-1}$, we can rewrite (7.15)-(7.17) as follows

$$
\begin{aligned}
& {\left[\begin{array}{ccr}
W_{i l} & \bullet & \bullet \\
\mathcal{H}_{i}^{(2)^{\prime}} J_{i l} & \mathcal{H}_{i}^{(2)^{\prime}} \mathcal{E}_{i}(P)^{-1} \mathcal{H}_{i}^{(2)} & \bullet \\
E_{i l}^{(2)} & 0 & I
\end{array}\right]>0,} \\
& \mathcal{T}_{i}^{(2)^{\prime}} P_{i} \mathcal{T}_{i}^{(2)}>\mathcal{T}_{i}^{(2)^{\prime}} \sum_{l \in \mathbb{M}_{i}} \alpha_{i l} M_{i l}^{(2)} \mathcal{T}_{i}^{(2)}, \\
& {\left[\begin{array}{ccr}
\mathcal{T}_{i}^{(2)^{\prime}} M_{i l}^{(2)} \mathcal{T}_{i}^{(2)} & \bullet & \bullet \\
\mathcal{H}_{i}^{(2)^{\prime}} A_{i l} \mathcal{T}_{i}^{(2)} & \mathcal{H}_{i}^{(2)^{\prime}} \mathcal{E}_{i}(P)^{-1} \mathcal{H}_{i}^{(2)} & \bullet \\
C_{i l}^{(2)} \mathcal{T}_{i}^{(2)^{\prime}} & 0 & I
\end{array}\right]>0,}
\end{aligned}
$$

hold, where $A_{i l}, J_{i l}, C_{i l}^{(2)}$, and $E_{i l}^{(2)}$ are the closed-loop matrices in (7.4). Applying the congruence transformations $\operatorname{diag}\left(I_{r} \mathcal{H}_{i}^{(2)^{-1}}, I_{q}\right), \mathcal{H}_{i}^{(2)^{-1}}$ and $\operatorname{diag}\left(\mathcal{T}_{i}^{(2)^{-1}}, \mathcal{H}_{i}^{(2)^{-1}}, I_{q}\right)$ to the previous inequalities, we get that (3.19) and (3.20) hold, and thus $\mathcal{C} \in \mathbb{C}$ and $\left\|\mathcal{G}_{c}\right\|_{2}<\gamma$.

For the case $(i i) \Longrightarrow(i)$, considering that $\hat{\theta}(k)=\theta(k)$ for all $k$, we have that $\alpha_{i i}=1$ for all $i \in \mathbb{N}$. In this case, the inequalities (3.19) and (3.20) reduce to $\sum_{i \in \mathbb{N}} \mu_{i} \operatorname{Tr}\left(W_{i}\right)<$ 
$\gamma^{2}$

$$
\left[\begin{array}{ccc}
W_{i} & \bullet & \bullet \\
J_{i i} & \mathcal{E}_{i}(P)^{-1} & \bullet \\
E_{i i}^{(2)} & 0 & I
\end{array}\right]>0,\left[\begin{array}{ccc}
P_{i} & \bullet & \bullet \\
A_{i i} & \mathcal{E}_{i}(P)^{-1} & \bullet \\
C_{i i}^{(2)} & 0 & I
\end{array}\right]>0,
$$

for a given controller $\mathcal{C} \in \mathbb{C}$, where $W_{i} \triangleq W_{i i}$. Applying the congruence transformations $\operatorname{diag}\left(I_{r}, \mathcal{H}_{i}^{(2)}, I_{q}\right)$ and $\operatorname{diag}\left(\mathcal{T}_{i}^{(2)}, \mathcal{H}_{i}^{(2)}, I_{q}\right)$ to the last two inequalities yields

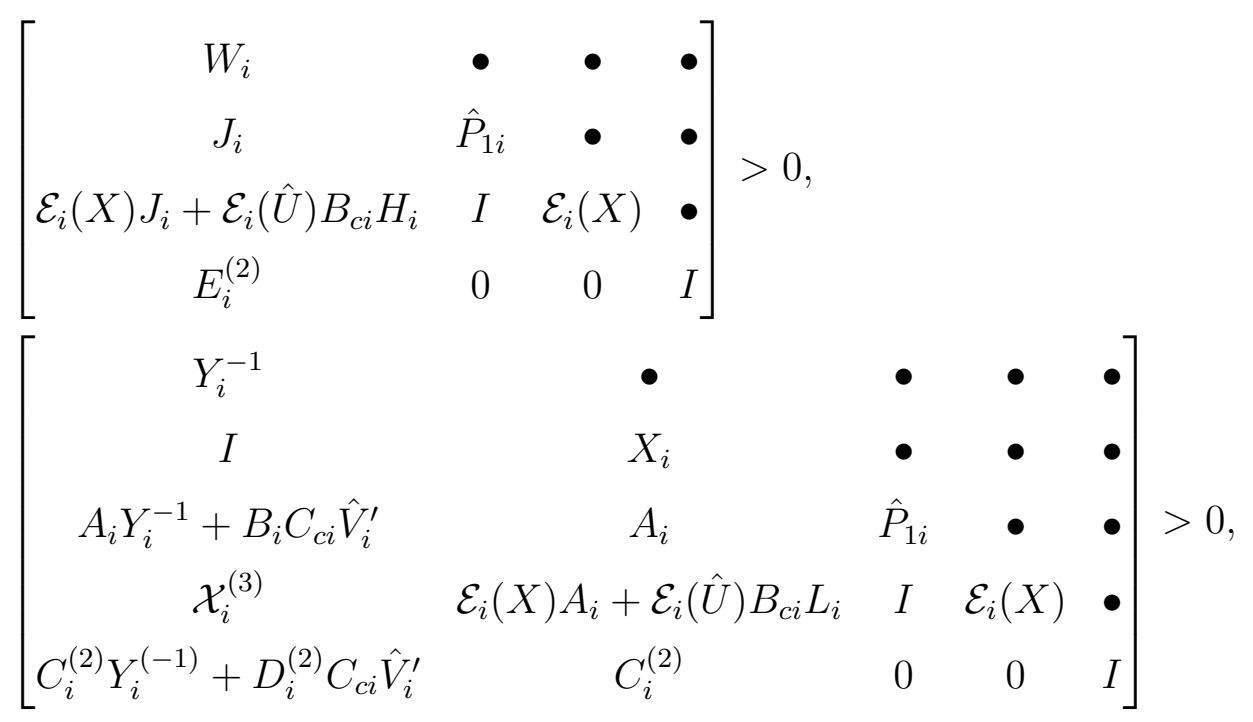

where $\mathcal{X}_{i}^{(3)} \triangleq \mathcal{E}_{i}(X) A_{i} Y_{i}^{-1}+\mathcal{E}_{i}(X) B_{i} C_{c i} \hat{V}_{i}^{\prime}+\mathcal{E}_{i}(\hat{U}) A_{c i} \hat{V}_{i}^{\prime}+\mathcal{E}_{i}(\hat{U}) B_{c i} L_{i} Y_{i}^{-1}$. Considering the reasoning explained in Section A.2, we have that $\mathcal{E}_{i}(Y)^{-1} \geq \hat{P}_{1 i}$, and then by applying the congruence transformations $\operatorname{diag}\left(I_{r}, \mathcal{E}_{i}(Y), I_{n}, I_{q}\right)$ and $\operatorname{diag}\left(Y_{i}, I_{n}, \mathcal{E}_{i}(Y), I_{n}, I_{q}\right)$, and defining $K_{i} \triangleq C_{c i} \hat{V}_{i}^{\prime} Y_{i}, F_{i} \triangleq \mathcal{E}_{i}(\hat{U}) B_{c i}$, and $R_{i} \triangleq \mathcal{E}_{i}(\hat{U}) A_{c i} \hat{V}_{i}^{\prime} Y_{i}$, we can write the following inequalities

$$
\begin{aligned}
& {\left[\begin{array}{cccc}
W_{i i} & \bullet & \bullet & \bullet \\
\mathcal{E}_{i}(Y) J_{i} & \mathcal{E}_{i}(Y) & \bullet & \bullet \\
\mathcal{E}_{i}(X) J_{i}+F_{i} H_{i} & \mathcal{E}_{i}(Y) & \mathcal{E}_{i}(X) & \bullet \\
E_{i}^{(2)} & 0 & 0 & I
\end{array}\right]>0,} \\
& {\left[\begin{array}{ccccc}
Y_{i} & & & & \\
Y_{i} & X_{i} & \bullet & \bullet & \bullet \\
\mathcal{E}_{i}(Y)\left[A_{i}+B_{i} K_{i}\right] & \mathcal{E}_{i}(Y) A_{i} & \mathcal{E}_{i}(Y) & \bullet & \bullet \\
\mathcal{E}_{i}(X)\left[A_{i}+B_{i} K_{i}\right]+F_{i} L_{i}+R_{i} & \mathcal{E}_{i}(X) A_{i}+F_{i} L_{i} & \mathcal{E}_{i}(Y) & \mathcal{E}_{i}(X) & \bullet \\
C_{i}^{(2)}+D_{i}^{(2)} K_{i} & C_{i}^{(2)} & 0 & 0 & I
\end{array}\right]>0 .}
\end{aligned}
$$


Defining $\mathcal{D}=\left[\begin{array}{cc}I & -I \\ 0 & I\end{array}\right]$ and applying the congruence transformations $\operatorname{diag}\left(I_{r}, \mathcal{D}, I_{q}\right)$ and $\operatorname{diag}\left(I_{2 n} \mathcal{D}, I_{q}\right)$ to the previous inequalities yields

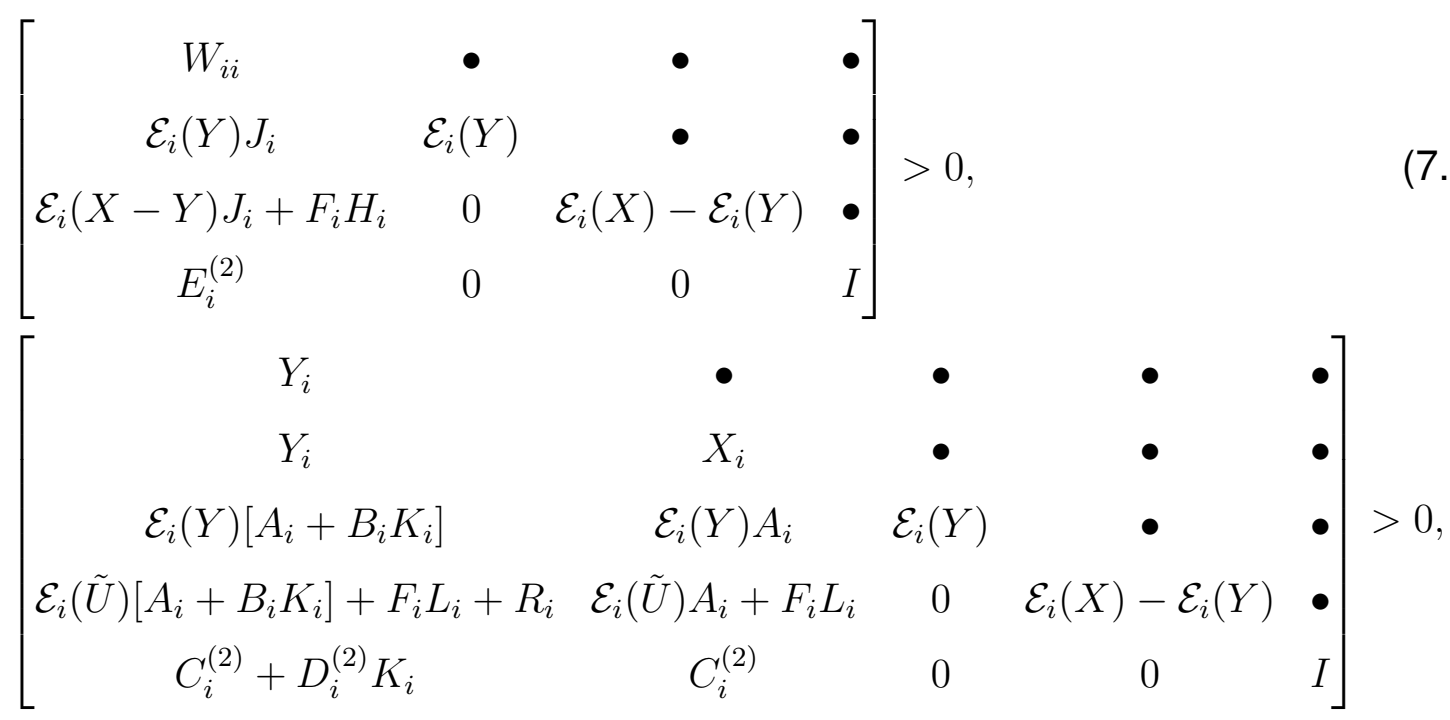

where $\tilde{U}_{i} \triangleq X_{i}-Y_{i}$, and thus, by recalling that $\alpha_{i i}=1$ in (7.7)-(7.10) and with the choice $G_{i}=\mathcal{E}_{i}(X-Y)$, we have that $(7.18)$ is equivalent to (7.8) and (7.19) is equivalent to (7.9)-(7.10).

Theorem 7.1 provides a sufficient result (that becomes also necessary in the modedependent case) in terms of bilinear matrix inequalities for the design of controllers depending only on $\hat{\theta}(k)$ by means of specific manipulations on the transformations presented in (GEROMEL; GONÇALVES; FIORAVANTI, 2009; GONÇALVES; FIORAVANTI; GEROMEL, 2010). It is also interesting to note the similarity of (7.7)-(7.10) with the design conditions for $\hat{\theta}(k)$-filters in Chapter 5, see Equations (5.8)-(5.10), by taking $K_{l}=0$ in (7.7)-(7.10). This similar pattern was also pointed out in (OLIVEIRA, 1999) for the continuous-time uncertain LTI case.

In the discussion that follows, we aim to provide a convex approximation for (7.7)(7.10). We recall the set of stabilizing state-feedback controllers $\mathbb{K}$ whose definition can be found in (4.5). The following lemma provides a relation between the solution set $\Xi_{2}$ and $\mathbb{K}$.

Lemma 7.1. If there exists $\xi_{2} \in \Xi_{2}$, then $K \in \mathbb{K}$.

Proof. It follows directly by analysing the $3 n \times 3 n$ diagonal block in (7.10). By applying 
the Schur complement to this block, we get that

$$
\left[\begin{array}{cc}
M_{i l}^{(2,1)}-A_{i l}^{(K)^{\prime}} \mathcal{E}_{i}(Y) A_{i l}^{(K)} & \bullet \\
M_{i l}^{(2,2)}-A_{i l}^{(K)^{\prime}} \mathcal{E}_{i}(Y) A_{i} & M_{i l}^{(2,3)}-A_{i}^{\prime} \mathcal{E}_{i}(Y) A_{i}
\end{array}\right]>0
$$

must hold, where $A_{i l}^{(K)} \triangleq A_{i}+B_{i} K_{l}$. Then, by considering (7.9), we get the stochastic stabilizability condition $Y_{i}>\sum_{l \in \mathbb{M}_{i}} \alpha_{i l} A_{i l}^{(K)^{\prime}} \mathcal{E}_{i}(Y) A_{i l}^{(K)}$, for all $i \in \mathbb{N}$.

Considering Theorem 7.1 and Lemma 7.1 and inspired in the ad hoc separation procedure of (OLIVEIRA, 1999; OLIVEIRA; GEROMEL; BERNUSSOU, 2000), we assume that the stabilizing state-feedback gain $K_{l}$ is given, and so the variable set of (7.7)-(7.10) for this case becomes

$$
\bar{\xi}_{2} \triangleq\left\{M_{i l}^{(2,1)}, M_{i l}^{(2,2)}, M_{i l}^{(2,3)}, Y_{i}, X_{i}, G_{l}, F_{l}, R_{l}, i \in \mathbb{N}, l \in \mathbb{M}_{i}\right\} \cup \xi_{a u x}^{(2)} .
$$

We also define

$$
\bar{\Xi}_{2}(K) \triangleq\left\{\bar{\xi}_{2}:(7.7)-(7.10) \text { hold }\right\}
$$

Corollary 7.1. For a given $K \in \mathbb{K}$ such that $\bar{\Xi}_{2}(K) \neq \emptyset$, if $\bar{\xi}_{2} \in \bar{\Xi}_{2}(K)$, then by setting $A_{c l}=-G_{l}^{-1} R_{l}, B_{c l}=-G_{l}^{-1} F_{l}$, and $C_{c l}=K_{l}$ for all $l \in \mathbb{M}$ we have that $\mathcal{C} \in \mathbb{C}$ and $\left\|\mathcal{G}_{c}\right\|_{2}<\gamma$.

Proof. It follows from Theorem 7.1.

The previous result deserves some comments. Note that by fixing $K$, the inequality (7.9) becomes an LMI in $\bar{\xi}_{2}$, and so (7.7)-(7.10) can be solved by standard LMI solvers. Then, the ad hoc separation procedure for MJLS with partial information consists in finding a stabilizing state-feedback gain and calculating the remaining controller matrices by Corollary 7.1. In this case, we can write the $\mathcal{H}_{2}$ dynamic output feedback control as follows

$$
\inf _{\bar{\xi}_{2} \in \bar{\Xi}_{2}(K)}\left\{\gamma_{a} ; \text { such that }(7.7)-(7.10) \text { hold }\right\} \text {. }
$$

The ad hoc separation procedure of Corollary 7.1 is shown in Algorithm 3. Considering the suboptimal characteristics of (7.20), it is a fact that those conditions do not parametrize all the controllers in the form (7.2) for the partial observation case. Besides, even if $K \in \Xi(K)$ in (7.7)-(7.10), we have no guarantee that $\bar{\Xi}_{2}(K) \neq \emptyset$ for our 
Algorithm 3 The $\mathcal{H}_{2}$ ad hoc separation procedure (Corollary 7.1)

1: Calculate a stochastic stabilizing state-feedback gain $K$ such that $\bar{\Xi}_{2}(K) \neq \emptyset$;

2: Use $K_{l}$ as an input in (7.7)-(7.10) and calculate $A_{c l}$ and $B_{c l}$ for all $l \in \mathbb{M}$. The final controller is given by $\left(A_{c l}, B_{c l}, C_{c l}\right)$ as shown in Corollary 7.1.

choice of $K$. In the case in which the conditions (7.7)-(7.10) do not hold for a given $K$, a new stabilizing state-feedback gain must be provided. As for the case where $\Xi_{2}=\emptyset$, there are no similar results in the literature to date that would provide an alternative to the design of $\mathcal{C}$ in the hidden MJLS formulation, and thus further study is required. Note also that contrarily to the two steps procedure of Chapter 6, the state-feedback gain $K$ is a part of the final structure, and not only a numerical means to obtain the desired controller.

Example 7.1: We consider the system of Chapter 6, specifically Example 6.1. We set $\rho_{1}=\rho_{2}=1$ and then, by using $K$ in (4.15) as an input to (7.20), we get the remaining controllers matrices

$$
\left[\begin{array}{c|c}
A_{c 1} & B_{c 1} \\
\hline A_{c 2} & B_{c 2}
\end{array}\right]=\left[\begin{array}{cc|c}
-0.9326 & 0.2334 & 0.8516 \\
-0.7588 & 0.5667 & 0.0183 \\
\hline 0.9000 & -0.0878 & 0.0878 \\
0.3000 & 0.7928 & 0.4072
\end{array}\right],
$$

with $r_{\sigma}(\mathcal{L})=0.4026$ and the optimal cost given by $\gamma_{c}^{*}=2.2507$. We now compare our result with respect to the separation principle of (COSTA; TUESTA, 2004), and thus we get that

$$
P_{1}=Y_{1}^{*}=\left[\begin{array}{rr}
2.0956 & -1.3341 \\
-1.3341 & 1.1814
\end{array}\right], P_{2}=Y_{2}^{*}=\left[\begin{array}{rr}
2.1577 & -0.8607 \\
-0.8607 & 1.9115
\end{array}\right],
$$

where $P_{i}$ is obtained by solving (B.1). We note that $\sum_{i \in \mathbb{N}} \mu_{i} \operatorname{Tr}\left(J_{i}^{\prime} \mathcal{E}_{i}\left(Y^{*}\right) J_{i}\right)=0.9741$, that is exactly the square of the cost provided by the state-feedback control part of the separation principle, $\gamma_{K}^{*}=0.987$. Conversely, by solving (B.3), we get

$$
S_{1}^{*}=\left[\begin{array}{ll}
1.3653 & 0.0824 \\
0.0824 & 0.3143
\end{array}\right], S_{2}^{*}=\left[\begin{array}{ll}
0.1801 & 0.0158 \\
0.0158 & 0.0511
\end{array}\right],
$$


for

$$
F_{1}(S)=\left[\begin{array}{l}
0.8516 \\
0.0183
\end{array}\right], F_{2}(S)=\left[\begin{array}{l}
0.0878 \\
0.4072
\end{array}\right]
$$

that are numerically equal to $B_{c i}$ calculated through (7.20). It is clear also that

$$
A_{c i}=A_{i}+B_{i} K_{i}-B_{c i} L_{i}
$$

for all $i \in \mathbb{N}$, echoing the result in (COSTA; TUESTA, 2004). Finally, by computing

$$
\left(\gamma_{F}^{*}\right)^{2}=\sum_{i \in \mathbb{N}} \operatorname{Tr}\left[O_{i}^{1 / 2} K_{i}\left(Y^{*}\right) S_{i} K_{i}\left(Y^{*}\right)^{\prime} O_{i}^{1 / 2}\right]=4.0914
$$

where $O_{i} \triangleq\left[B_{i}^{\prime} \mathcal{E}_{i}(P) B_{i}+D_{i}^{\prime} D_{i}\right]$, we have that $\left(\gamma_{c}^{*}\right)^{2}=\left(\gamma_{K}^{*}\right)^{2}+\left(\gamma_{F}^{*}\right)^{2}=0.9741+4.0914=$ 5.0655 , that is, $\left\|\mathcal{G}_{*}\right\|_{2}=2.2507$, as expected, due to the necessity of Theorem 7.1 and the choice of $K$.

We now set $\rho=0.7$ and use the state-feedback gains given in (4.16) for calculating the final dynamic controller given by

$$
\left[\begin{array}{l|l}
A_{c 1} & B_{c 1} \\
\hline A_{c 2} & B_{c 2}
\end{array}\right]=\left[\begin{array}{rr|r}
-0.6145 & -0.0315 & 0.9231 \\
-0.6741 & 0.6821 & 0.2050 \\
\hline 0.0205 & -0.0281 & 0.5557 \\
-0.4236 & 0.9857 & 0.2079
\end{array}\right],
$$

with $r_{\sigma}(\mathcal{L})=0.7658, \gamma^{*}=3.8978$, and $\left\|\mathcal{G}_{c}\right\|_{2}=2.9660$. 


\section{3 $\mathcal{H}_{\infty}$ dynamic output feedback control for hidden MJLS}

Consider the following inequalities,

$$
\begin{aligned}
& {\left[\begin{array}{ccc}
Z_{i} & \bullet & \bullet \\
Z_{i} & T_{i} & \bullet \\
0 & 0 & \delta^{2} I
\end{array}\right]>\sum_{l \in \mathbb{M}_{i}} \alpha_{i l}\left[\begin{array}{ccc}
M_{i l}^{(o, 1)} & \bullet & \bullet \\
M_{i l}^{(o, 2)} & M_{i l}^{(o, 3)} & \bullet \\
N_{i l}^{(o, 1)} & N_{i l}^{(o, 2)} & S_{i l}^{(o, 1)}
\end{array}\right],} \\
& {\left[\begin{array}{cccccc}
M_{i l}^{(o, 1)} & \bullet & \bullet & \bullet & \bullet & \bullet \\
M_{i l}^{(o, 2)} & M_{i l}^{(o, 3)} & \bullet & \bullet & \bullet & \bullet \\
N_{i l}^{(o, 1)} & N_{i l}^{(o, 2)} & S_{i l}^{(o, 1)} & \bullet & \bullet & \bullet \\
\mathcal{E}_{i}(Z)\left(A_{i}+B_{i} K_{l}\right) & \mathcal{E}_{i}(Z) A_{i} & \mathcal{E}_{i}(Z) J_{i} & \mathcal{E}_{i}(Z) & \bullet & \bullet \\
G_{l}\left(A_{i}+B_{i} K_{l}\right)+F_{l} L_{i}+R_{l} & G_{l} A_{i}+F_{l} L_{i} & G_{l} J_{i}+F_{l} H_{i} & 0 & \Phi_{i l} & \bullet \\
C_{i}^{(\infty)}+D_{i}^{(\infty)} K_{l} & C_{i}^{(\infty)} & E_{i}^{(\infty)} & 0 & 0 & I
\end{array}\right]>0,}
\end{aligned}
$$

for all $i \in \mathbb{N}, l \in \mathbb{M}_{i}$, where $\Phi_{i l}=\operatorname{Her}\left(G_{l}\right)+\mathcal{E}_{i}(Z)-\mathcal{E}_{i}(T)$. We define the set of variables of $(7.21)-(7.22)$ as

$\xi_{\infty} \triangleq\left\{M_{i l}^{(o, 1)}, M_{i l}^{(o, 2)}, M_{i l}^{(o, 3)}, N_{i l}^{(o, 1)}, N_{i l}^{(o, 2)}, S_{i l}^{(o, 1)}, Z_{i}, T_{i}, G_{l}, K_{l}, F_{l}, R_{l}, i \in \mathbb{N}, l \in \mathbb{M}_{i}\right\} \cup \xi_{\text {aux }}^{(\infty)}$,

where $\xi_{\text {aux }}^{(\infty)}=\emptyset$ if $\delta$ is given, and $\xi_{\text {aux }}^{(\infty)}=\delta_{a}, \delta_{a} \triangleq \delta^{2}$. The set of all solutions of (7.21)(7.22) is given by

$$
\Xi_{\infty} \triangleq\left\{\xi_{\infty}:(7.21)-(7.22) \text { holds }\right\}
$$

Theorem 7.2. Consider the following statements:

(i) There exists $\xi_{\infty}$ such that $\xi_{\infty} \in \Xi_{\infty}$.

(ii) There exists a controller $\mathcal{C}$ such that $\mathcal{C} \in \mathbb{C}$ and $\left\|\mathcal{G}_{c}\right\|_{\infty}<\delta$.

We have that $(i) \Longrightarrow$ (ii) by setting $A_{c l}=-G_{l}^{-1} R_{l}, B_{c l}=-G_{l}^{-1} F_{l}$, and $C_{c l}=K_{l}$ for all $l \in \mathbb{M}$. Moreover $(i i) \Longrightarrow(i)$ whenever $\hat{\theta}=\theta$.

Proof. The proof is similar to the one given for Theorem 7.1. For the implication (ii) $\Longrightarrow(i)$, we note that $G_{l}$ is non-singular due to $\phi_{i l}>0$ in (7.22). Then, by setting $R_{l}=-G_{l} A_{c l}, F_{l}=-G_{l} B_{c l}$, and $K_{l}=C_{c l}$ in (7.22), as well as recalling Lemma 
A.2 allow us to write that

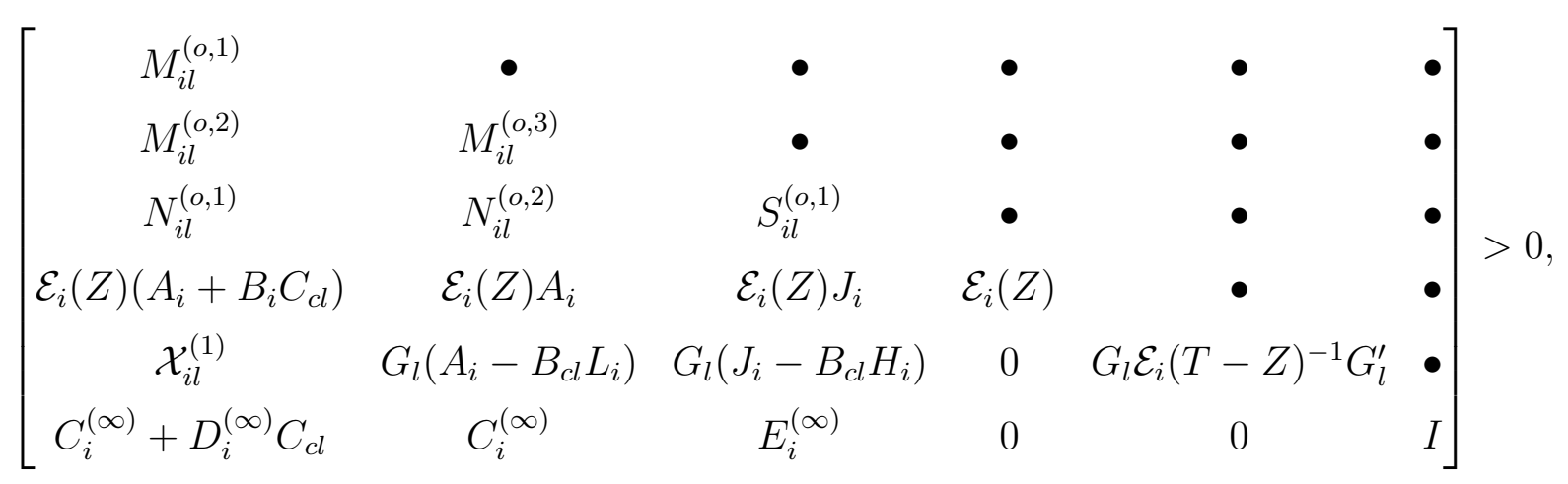

holds, where $\mathcal{X}_{i l}^{(1)} \triangleq G_{l}\left(A_{i}+B_{i} C_{c l}-B_{c l} L_{i}-A_{c l}\right)$. We define the matrix

$$
\mathcal{D}_{i l} \triangleq\left[\begin{array}{cc}
I & I \\
0 & G_{l}^{-T} \mathcal{E}_{i}(T-Z)
\end{array}\right]
$$

and apply the congruence transformation $\operatorname{diag}\left(I_{2 n}, I_{r}, \mathcal{D}_{i l}, I_{q}\right)$ to the last inequality in order to obtain that

$$
\left[\begin{array}{cccccc}
M_{i l}^{(o, 1)} & \bullet & \bullet & \bullet & \bullet & \bullet \\
M_{i l}^{(o, 2)} & M_{i l}^{(o, 3)} & \bullet & \bullet & \bullet & \bullet \\
N_{i l}^{(o, 1)} & N_{i l}^{(o, 2)} & S_{i l}^{(o, 1)} & \bullet & \bullet & \bullet \\
\mathcal{E}_{i}(Z)\left(A_{i}+B_{i} C_{c l}\right) & \mathcal{E}_{i}(Z) A_{i} & \mathcal{E}_{i}(Z) J_{i} & \mathcal{E}_{i}(Z) & \bullet & \bullet \\
\mathcal{X}_{i l}^{(2)} & \mathcal{X}_{i l}^{(3)} & \mathcal{E}_{i}(T) J_{i}+\mathcal{E}_{i}(Z-T) B_{c l} H_{i} & \mathcal{E}_{i}(Z) & \mathcal{E}_{i}(T) & \bullet \\
C_{i}^{(\infty)}+D_{i}^{(\infty)} C_{c l} & C_{i}^{(\infty)} & E_{i}^{(\infty)} & 0 & 0 & I
\end{array}\right]>0
$$

holds, where $\mathcal{X}_{i l}^{(2)} \triangleq \mathcal{E}_{i}(X)\left[A_{i}+B_{i} C_{c l}\right]+\mathcal{E}_{i}(Z-T) B_{c l} L_{i}+\mathcal{E}_{i}(Z-T) A_{c l}$ and $\mathcal{X}_{i l}^{(3)} \triangleq$ $\mathcal{E}_{i}(T) A_{i}+\mathcal{E}_{i}(Z-T) B_{c l} L_{i}$. Applying the congruence transformations $\operatorname{diag}\left(Z_{i}^{-1}, I_{n}, I_{r}\right)$ to (7.21) and $\operatorname{diag}\left(Z_{i}^{-1}, I_{n}, I_{r}, \mathcal{E}_{i}(Z)^{-1}, I_{n}, I_{q}\right)$ to the last inequality and defining $U_{i} \triangleq Z_{i}-T_{i}$ allow us to write that

$$
\left[\begin{array}{ccc}
Z_{i}^{-1} & \bullet & \bullet \\
I & T_{i} & \bullet \\
0 & 0 & \delta^{2} I
\end{array}\right]>\sum_{l \in \mathbb{M}_{i}} \alpha_{i l}\left[\begin{array}{ccc}
\bar{M}_{i l}^{(o, 1)} & \bullet & \bullet \\
\bar{M}_{i l}^{(o, 2)} & M_{i l}^{(o, 3)} & \bullet \\
\bar{N}_{i l}^{(o, 1)} & N_{i l}^{(o, 2)} & S_{i l}^{(o, 1)}
\end{array}\right]
$$




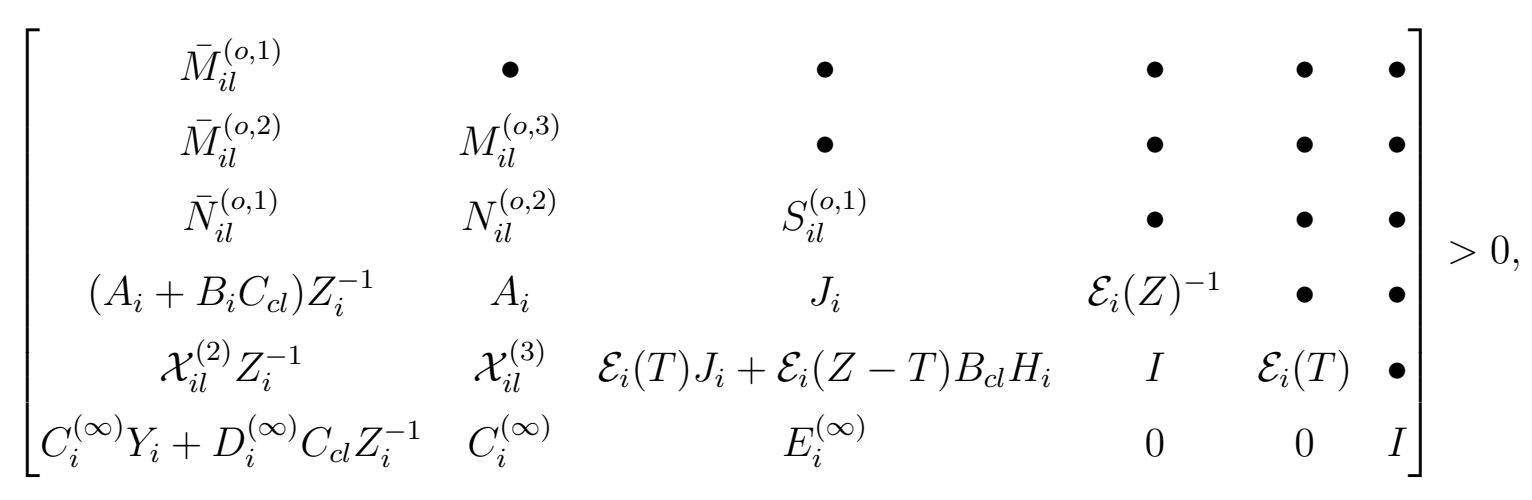

holds for all $i \in \mathbb{N}$. By imposing the structures

$$
Q_{i}=\left[\begin{array}{cc}
T_{i} & Z_{i}-T_{i} \\
Z_{i}-T_{i} & T_{i}-Z_{i}
\end{array}\right]
$$

and

$$
M_{i l}^{(\infty)}=\left[\begin{array}{cc}
M_{i l}^{(o, 3)} & \bullet \\
M_{i l}^{(o, 2)^{\prime}}-M_{i l}^{(o, 3)} & M_{i l}^{(o, 1)}-\operatorname{Her}\left(M_{i l}^{(o, 2)}\right)+M_{i l}^{(o, 3)}
\end{array}\right], N_{i l}^{(\infty)^{\prime}}=\left[\begin{array}{c}
N_{i l}^{(o, 2)^{\prime}} \\
N_{i l}^{(o, 1)^{\prime}}-N_{i l}^{(o, 2)^{\prime}}
\end{array}\right],
$$

and $S_{i l}^{(\infty)}=S_{i l}^{(o, 1)}$ in (3.26), we get that $\bar{U}_{i}=-\hat{T}_{i}=Z_{i}-T_{i}$ in (A.2) and recalling the discussion in Appendix A, we have that $\bar{V}_{i}=\bar{V}_{i}^{\prime}=Z_{i}^{-1}$. Besides, by bearing in mind the definitions of $\mathcal{T}_{i}^{(\infty)}$ and $\mathcal{H}_{i}^{(\infty)}$ in (A.9) and (A.10)

$$
\mathcal{T}_{i}^{(\infty)}=\left[\begin{array}{cc}
Z_{i}^{-1} & I \\
\bar{V}_{i}^{\prime} & 0
\end{array}\right] \text { such that } \mathcal{T}_{i}^{(\infty)^{\prime}} P_{i} \mathcal{T}_{i}^{(\infty)}=\left[\begin{array}{cc}
Z_{i}^{-1} & I \\
I & T_{i}
\end{array}\right]
$$

as well as

$$
\mathcal{H}_{i}^{(\infty)}=\left[\begin{array}{ll}
I & \mathcal{E}_{i}(T) \\
0 & \mathcal{E}_{i}(\bar{U})^{\prime}
\end{array}\right] \text { such that } \mathcal{H}_{i}^{(\infty)^{\prime}} \mathcal{E}_{i}(P)^{-1} \mathcal{H}_{i}^{(\infty)}=\left[\begin{array}{cc}
\hat{Q}_{1 i} & I \\
I & \mathcal{E}_{i}(T)
\end{array}\right],
$$

with $\bar{U}_{i}=Z_{i}-T_{i}$ and $\hat{Q}_{1 i}=\left[\mathcal{E}_{i}(T)-\mathcal{E}_{i}(\bar{U}) \mathcal{E}_{i}(\hat{T})^{-1} \mathcal{E}_{i}(\bar{U})^{\prime}\right]^{-1}=\mathcal{E}_{i}(T+\bar{U})^{-1}=\mathcal{E}_{i}(Z)^{-1}$, we can rewrite A.2 (7.25) and (7.26) as follows

$$
\left[\begin{array}{cc}
\mathcal{T}_{i}^{(\infty)}{ }^{\prime} Q_{i} \mathcal{T}_{i}^{(\infty)} & \bullet \\
0 & \delta^{2} I
\end{array}\right]>\left[\begin{array}{cc}
\mathcal{T}_{i}^{(\infty)}{ }^{\prime} M_{i l} \mathcal{T}_{i}^{(\infty)} & \bullet \\
N_{i l} \mathcal{T}_{i}^{(\infty)} & S_{i l}
\end{array}\right]
$$




$$
\left[\begin{array}{cccc}
\mathcal{T}_{i}^{(\infty)^{\prime}} M_{i l} \mathcal{T}_{i}^{(\infty)} & \bullet & \bullet & \bullet \\
N_{i l} \mathcal{T}_{i}^{(\infty)} & S_{i l} & \bullet & \bullet \\
\mathcal{H}_{i}^{(\infty)^{\prime}} A_{i l} \mathcal{T}_{i}^{(\infty)} & \mathcal{H}_{i}^{(\infty)^{\prime}} J_{i l} & \mathcal{H}_{i}^{(\infty))^{\prime}} \mathcal{E}_{i}(Q)^{-1} \mathcal{H}_{i}^{(\infty)} & \bullet \\
C_{i l}^{(\infty)} \mathcal{T}_{i}^{(\infty)} & E_{i}^{(\infty)} & 0 & I
\end{array}\right]>0
$$

where $A_{i l}, J_{i l}, C_{i l}^{(\infty)}$, and $E_{i l}^{(\infty)}$ are the closed-loop matrices in (7.4). By respectively applying the congruence transformations $\operatorname{diag}\left(\mathcal{T}_{i}^{(\infty)^{-1}}, I_{r}\right)$ and $\operatorname{diag}\left(\mathcal{T}_{i}^{(\infty)^{-1}}, I_{r}, \mathcal{H}_{i}^{(\infty)^{-1}}, I_{q}\right)$, we get the conditions of the bounded-real lemma in (3.26), and therefore the claim follows.

For $(i) \Longrightarrow(i i)$, we have that $\hat{\theta}(k)=\theta(k)$ that implies that $\alpha_{i i}=1$ for all $i \in \mathbb{N}$. This allows us to rewrite the conditions of the bounded-real lemma as follows:

$$
\left[\begin{array}{cccc}
Q_{i} & \bullet & \bullet & \bullet \\
0 & \delta^{2} I & \bullet & \bullet \\
A_{i i} & J_{i i} & \mathcal{E}_{i}(Q)^{-1} & \bullet \\
C_{i i}^{(\infty)} & E_{i i}^{(\infty)} & 0 & I
\end{array}\right]>0,
$$

for all $i \in \mathbb{N}$, for the closed-loop matrices in (7.4). By respectively applying the congruence transformation $\operatorname{diag}\left(\mathcal{T}_{i}^{(\infty)}, I_{r}, \mathcal{H}_{i}^{(\infty)}, I_{q}\right)$, we get that

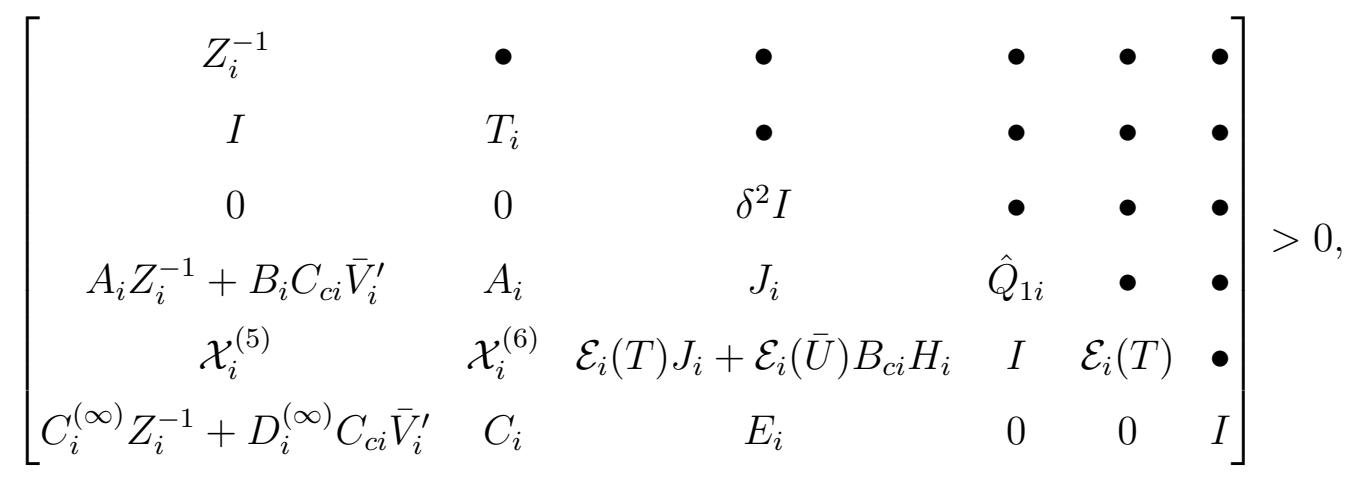

where $\mathcal{X}_{i}^{(5)} \triangleq \mathcal{E}_{i}(T) A_{i} Z_{i}^{-1}+\mathcal{E}_{i}(T) B_{i} C_{c i} \bar{V}_{i}^{\prime}+\mathcal{E}_{i}(\bar{U}) B_{c i} L_{i} Z_{i}^{-1}+\mathcal{E}_{i}(\bar{U}) A_{c i} V_{i}^{\prime}$ and $\mathcal{X}_{i}^{(6)} \triangleq$ $\mathcal{E}_{i}(T) A_{i}+\mathcal{E}_{i}(\bar{U}) B_{c i} L_{i}$. Recalling that $\mathcal{E}_{i}(Z)^{-1} \geq \hat{Q}_{1 i}$ (see Section A.2), we rewrite the previous inequality, after applying the congruence transformation $\operatorname{diag}\left(Z_{i}, I_{n}, I_{r}, \mathcal{E}_{i}(Z), I_{q}\right)$, 
as follows

$$
\left[\begin{array}{cccccc}
Z_{i} & \bullet & \bullet & \bullet & \bullet & \bullet \\
Z_{i} & T_{i} & \bullet & \bullet & \bullet & \bullet \\
0 & 0 & \delta^{2} I & \bullet & \bullet & \bullet \\
\mathcal{E}_{i}(Z)\left(A_{i}+B_{i} K_{i}\right) & \mathcal{E}_{i}(Z) A_{i} & \mathcal{E}_{i}(Z) J_{i} & \mathcal{E}_{i}(Z) & \bullet & \bullet \\
\mathcal{X}_{i}^{(7)} & \mathcal{E}_{i}(T) A_{i}+F_{i} L_{i} & \mathcal{E}_{i}(T) J_{i}+F_{i} H_{i} & \mathcal{E}_{i}(Z) & \mathcal{E}_{i}(T) & \bullet \\
C_{i}^{(\infty)}+D_{i}^{(\infty)} K_{i} & C_{i}^{(\infty)} & E_{i}^{(\infty)} & 0 & 0 & I
\end{array}\right]>0,
$$

where $\mathcal{X}_{i}^{(7)} \triangleq \mathcal{E}_{i}(T)\left(A_{i}+B_{i} K_{i}\right)+F_{i} L_{i}+R_{i}, R_{i}=\mathcal{E}_{i}(\bar{U}) A_{c i} \bar{V}_{i}^{\prime} Z_{i}, F_{i}=\mathcal{E}_{i}(\bar{U}) B_{c i}$, and $K_{i}=C_{c i} \bar{V}_{i}^{\prime} Z_{i}$. By defining $\mathcal{D}=\left[\begin{array}{cc}I & -I \\ 0 & I\end{array}\right]$ and applying the congruence transformation $\operatorname{diag}\left(I_{2 n}, I_{r}, \mathcal{D}, I_{q}\right)$ in the previous inequality, we get

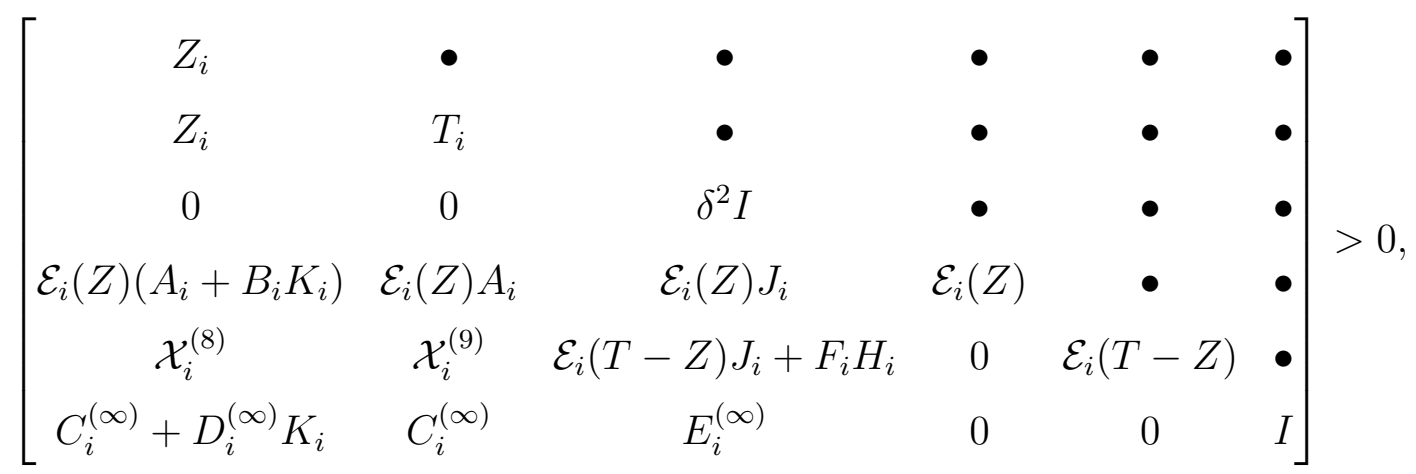

where $\mathcal{X}_{i}^{(8)} \triangleq \mathcal{E}_{i}(T-Q)\left(A_{i}+B_{i} K_{i}\right)+F_{i} L_{i}+R_{i}$ and $\mathcal{X}_{i}^{(9)} \triangleq \mathcal{E}_{i}(T-Z) A_{i}+F_{i} L_{i}$. Finally, by noting that for $\alpha_{i i}$ and the choice $G_{i}=\mathcal{E}_{i}(T-Z)$ we get that (7.22) is equivalent to (7.27), and thus the claim follows.

Following the similar arguments as those previously applied to the $\mathcal{H}_{2}$ control, we can enunciate the following lemma.

Lemma 7.2. If there exists $\xi_{\infty} \in \Xi_{\infty}$, then $K \in \mathbb{K}$.

Proof. It follows the similar reasoning as used in the proof of Lemma 7.2.

We define the set of of variables of (7.21)-(7.22) for a given $K$ as follows $\bar{\xi}_{\infty} \triangleq\left\{M_{i l}^{(o, 1)}, M_{i l}^{(o, 2)} M_{i l}^{(o, 3)}, N_{i l}^{(o, 1)}, N_{i l}^{(o, 2)}, S_{i l}^{(o, 1)}, Z_{i}, T_{i}, G_{l}, F_{l}, R_{l}, i \in \mathbb{N}, l \in \mathbb{M}_{i}\right\} \cup \xi_{\text {aux }}^{(\infty)}$ 
along with

$$
\bar{\Xi}_{\infty}(K) \triangleq\left\{\bar{\xi}_{\infty}:(7.21)-(7.22) \text { hold }\right\}
$$

Corollary 7.2. For a given $K \in \mathbb{K}$ such that $\bar{\Xi}_{\infty}(K) \neq \emptyset$, if $\bar{\xi}_{\infty} \in \bar{\Xi}_{\infty}(K)$, then by setting $A_{c l}=-G_{l}^{-1} R_{l}, B_{c l}=-G_{l}^{-1} F_{l}$, and $C_{c l}=K_{l}$ for all $l \in \mathbb{M}$ we have that $\mathcal{C} \in \mathbb{C}$ and $\left\|\mathcal{G}_{c}\right\|_{\infty}<\delta$.

Proof. It follows from Theorem 7.2.

Corollary 7.2 allows us to write the following $\mathcal{H}_{\infty}$ control problem in a convex formulation

$$
\inf _{\bar{\xi}_{\infty} \in \bar{\Xi}_{\infty}(K)}\left\{\delta_{a} ; \text { such that }(7.21)-(7.22) \text { hold }\right\},
$$

where $\delta_{a}=\delta^{2}$. The separation procedure of Corollary 7.2 is shown in Algorithm 4.

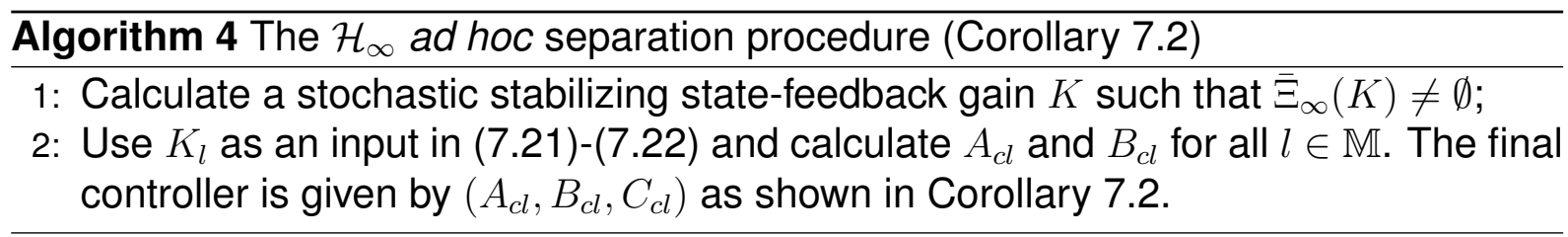

Example 7.2: As an illustrative example, we consider the same system as described in Examples 4.3 and 7.1. We set $\rho=1$, calculate the optimal $\mathcal{H}_{\infty}$ state-feedback control by means of problem (4.21), and use $K$ shown in (4.22) as an input to (7.28), in order to get the remaining controllers matrices

$$
\left[\begin{array}{l|l}
A_{c 1} & B_{c 1} \\
\hline A_{c 2} & B_{c 2}
\end{array}\right]=\left[\begin{array}{rr|r}
-1.2743 & -0.0201 & 1.4221 \\
-0.7319 & 0.4426 & 0.1067 \\
\hline 1.0725 & -0.3511 & 0.3121 \\
0.3072 & 0.5907 & 0.7129
\end{array}\right],
$$

with the $\mathcal{H}_{\infty}$ cost given by $\delta_{c}=3.8999$ and $\left\|\mathcal{G}_{c}\right\|_{\infty}=3.6010$, that is, with the optimal $\mathcal{H}_{\infty}$ state-feedback used as an input to (7.28), we get a sub-optimal $\mathcal{H}_{\infty}$ dynamic output feedback controller. On the other hand, with the choice

$$
\left[\begin{array}{l}
K_{1} \\
K_{2}
\end{array}\right]=\left[\begin{array}{cc}
-0.5760 & -0.0819 \\
\hline 0 & 0
\end{array}\right]
$$


in (7.28), we get

$$
\left[\begin{array}{l|l}
A_{c 1} & B_{c 1} \\
\hline A_{c 2} & B_{c 2}
\end{array}\right]=\left[\begin{array}{rr|r}
-1.2235 & -0.0698 & 1.4869 \\
-0.7007 & 0.4267 & 0.1120 \\
\hline 1.0928 & -0.3788 & 0.3437 \\
0.3134 & 0.5555 & 0.7654
\end{array}\right]
$$

with $r_{\sigma}(\mathcal{L})=0.4124$, and $\gamma_{c}^{*}=\left\|\mathcal{G}_{c}\right\|_{\infty}=3.5953$, that coincides with the result in (GEROMEL; GONÇALVES; FIORAVANTI, 2009) for strictly proper controllers.

We now set $\rho=0.7$, calculate the $\mathcal{H}_{\infty}$ state-feedback controller through (4.21), and use $K$ in (4.23) to finally get the remaining controller matrices

$$
\left[\begin{array}{c|c}
A_{c 1} & B_{c 1} \\
\hline A_{c 2} & B_{c 2}
\end{array}\right]=\left[\begin{array}{rr|r}
-0.4741 & -0.2902 & 0.9944 \\
-0.2997 & 0.2872 & -0.0269 \\
\hline 0.2415 & -0.3517 & 0.4192 \\
-0.0859 & 0.4735 & -0.0456
\end{array}\right],
$$

with $r_{\sigma}(\mathcal{L})=0.8002, \delta^{*}=16.9927$, and $\left\|\mathcal{G}_{c}\right\|_{\infty}=10.2283$.

\subsection{The mixed $\mathcal{H}_{2} / \mathcal{H}_{\infty}$ dynamic output feedback control}

Theorems 7.1 and 7.2 allow us to state a new result that presents conditions for achieving dynamic controllers such that $\mathcal{C} \in \mathbb{C},\left\|\mathcal{G}_{c}\right\|_{2}<\gamma$, and $\left\|\mathcal{G}_{c}\right\|_{\infty}<\delta$. For that, define

$$
\bar{\xi} \triangleq \bar{\xi}_{(2)} \cup \bar{\xi}_{(\infty)}
$$

along with

$$
\bar{\Xi}(K) \triangleq\{\bar{\xi} \text { such that the LMIs }(7.7)-(7.10) \text { and }(7.21)-(7.22) \text { are jointly feasible }\} \text {. }
$$

Theorem 7.3. For a given $K \in \mathbb{K}$ such that $\bar{\Xi}(K) \neq \emptyset$, if $\bar{\xi} \in \bar{\Xi}(K)$, then by setting $A_{c l}=-G_{l}^{-1} R_{l}, B_{c l}=-G_{l}^{-1} F_{l}$, and $C_{c l}=K_{l}$ for all $l \in \mathbb{M}$, we have that $\mathcal{C} \in \mathbb{C},\left\|\mathcal{G}_{c}\right\|_{2}<\gamma$, and $\left\|\mathcal{G}_{c}\right\|_{\infty}<\delta$.

Proof. The result is a consequence of Theorems 7.1 and 7.2 and the use of a common same slack variable $G_{l}$, and matrices $R_{l}, F_{l}$, and $K_{l}$. 
In this case, we rewrite the optimization problem (7.6) as follows:

$$
\inf _{\bar{\xi} \in \bar{\Xi}(K)} g(\gamma, \delta) .
$$

Remark 7.1: The property described in Corollary 3.2 enables us to simplify problem (7.29) given that $z_{2}=z_{\infty}$. In this case, by setting $C_{\theta(k)}=C_{\theta(k)}^{(\infty)}=C_{\theta(k)}^{(2)}$, $D_{\theta(k)}=D_{\theta(k)}^{(\infty)}=D_{\theta(k)}^{(2)}$, and $E_{\theta(k)}=E_{\theta(k)}^{(\infty)}=E_{\theta(k)}^{(2)}$, as well as $Y_{i}=Z_{i}$ and $X_{i}=T_{i}$, it is possible to redefine $\bar{\phi}$ as $\bar{\xi}=\bar{\xi}_{\infty} \cup\left\{W_{i l}, i \in \mathbb{N}, l \in \mathbb{M}_{i}\right\}$ and $\bar{\Xi}=\{\bar{\xi}$ such that (7.7) (7.8) and (7.21) - (7.22) jointly hold $\}$ in (7.29) and Theorem 7.3 would still hold, albeit a more conservative result would be obtained.

Example 7.3: We use the same system given in Example 7.1. For the complete observation case $\rho=1.0$, we use the $\mathcal{H}_{\infty}$ state-feedback controller in (4.22) as an input to (7.29) and minimize $\gamma$ subject to $\delta=10$. The resulting controller is given below

$$
\left[\begin{array}{l|l}
A_{c 1} & B_{c 1} \\
\hline A_{c 2} & B_{c 2}
\end{array}\right]=\left[\begin{array}{rr|r}
-0.7031 & -0.0910 & 0.8904 \\
-0.3605 & 0.1596 & -0.1448 \\
\hline 0.9000 & -0.0970 & 0.0970 \\
0.3000 & 0.7941 & 0.4059
\end{array}\right],
$$

along with $K$ in (4.22), for $r_{\sigma}(\mathcal{L})=0.5112, \gamma_{c}^{*}=2.5479,\left\|\mathcal{G}_{c}\right\|_{2}=2.2895, \delta_{c}^{*}=10$, and $\left\|\mathcal{G}_{c}\right\|_{\infty}=4.6936$. On the other hand, for $\rho=0.7$, we use the controllers in (4.23) as an input to (7.29). By selecting the upper bound $\delta^{2}=500$ and minimizing $\gamma$ in (7.29), the resulting mixed $\mathcal{H}_{2} / \mathcal{H}_{\infty}$ dynamic controller is given below

$$
\left[\begin{array}{r|r}
A_{c 1} & B_{c 1} \\
\hline A_{c 2} & B_{c 2}
\end{array}\right]=\left[\begin{array}{rr|r}
-0.4044 & -0.3116 & 0.8885 \\
-0.1967 & 0.2778 & -0.0873 \\
\hline 0.1794 & -0.3161 & 0.4834 \\
-0.0160 & 0.4858 & -0.0562
\end{array}\right],
$$

with $r_{\sigma}(\mathcal{L})=0.8076, \gamma_{c}^{*}=4.4629,\left\|\mathcal{G}_{c}\right\|_{2}=3.2270, \delta_{c}=22.3607$, and $\left\|\mathcal{G}_{c}\right\|_{\infty}=10.9459$.

\subsection{Dynamic output feedback control for the cluster case and robust control}

Similarly as it was done in the previous sections, it is possible to investigate the cluster case through our formulation. In this case, we introduce the following inequality 
sets

$$
\begin{aligned}
& \sum_{i \in \mathbb{N}} \mu_{i} \operatorname{Tr}\left(\bar{W}_{i}\right)<\gamma^{2}, \\
& {\left[\begin{array}{cccc}
\bar{W}_{i} & \bullet & \bullet & \bullet \\
\mathcal{E}_{i}(Y) J_{i} & \mathcal{E}_{i}(Y) & \bullet & \bullet \\
G^{s} J_{i}+F^{s} H_{i} & 0 & \Psi_{i}^{s} & \bullet \\
E_{i}^{(2)} & 0 & 0 & I
\end{array}\right]>0,} \\
& {\left[\begin{array}{cccccc}
Y_{i} & & & & & \\
{\left[Y_{i}\right.} & & X_{i} & \bullet & \bullet & \bullet \\
\mathcal{E}_{i}(Y)\left(A_{i}+B_{i} K^{s}\right) & \mathcal{E}_{i}(Y) A_{i} & \mathcal{E}_{i}(Y) & \bullet & \bullet \\
G^{s}\left(A_{i}+B_{i} K^{s}\right)+F^{s} L_{i}+R^{s} & G^{s} A_{i}+F^{s} L_{i} & 0 & \Psi_{i}^{s} & \bullet \\
C_{i}^{(2)}+D_{i}^{(2)} K^{s} & C_{i}^{(2)} & 0 & 0 & I
\end{array}\right]>0,}
\end{aligned}
$$

where $\Psi_{i}^{s} \triangleq \operatorname{Her}\left(G^{s}\right)+\mathcal{E}_{i}(Y)-\mathcal{E}_{i}(X)$, along with

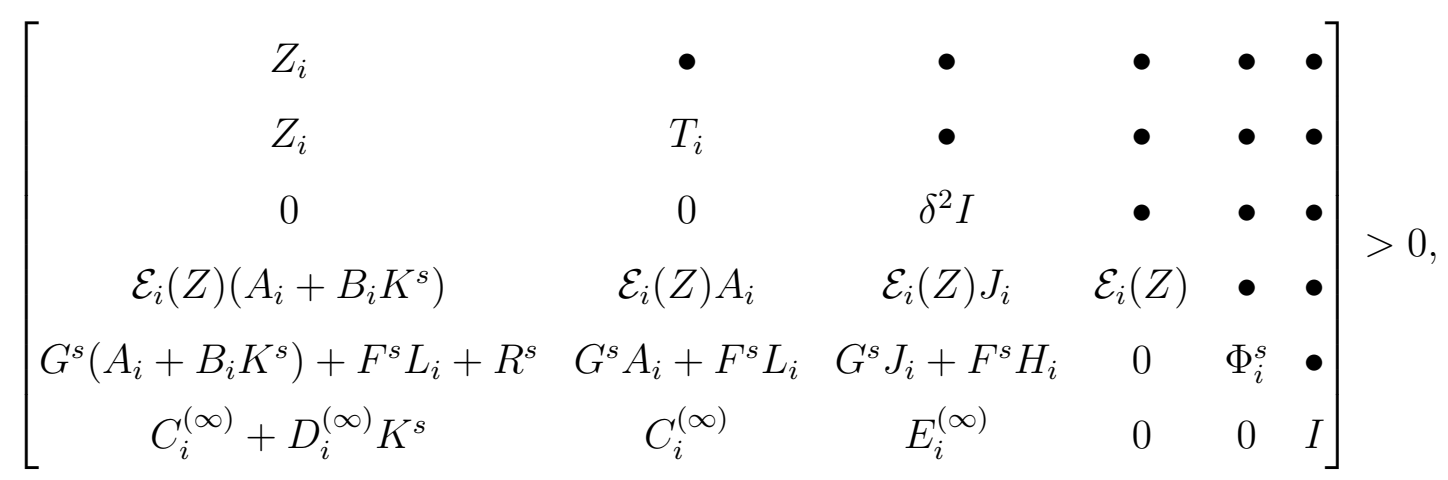

for $s \in\{1, \ldots, \kappa\}, i \in \mathbb{N}^{s}$, where $\Phi_{i}^{s} \triangleq \operatorname{Her}\left(G^{s}\right)+\mathcal{E}_{i}(Z)-\mathcal{E}_{i}(T)$.

Proposition 7.1: Under Assumption 3.1, for a given $K_{l}=K^{s}, l \in \mathbb{M}^{s}, s \in\{1, \ldots, \kappa\}$, such that $K \in \mathbb{K}$, we have that

- Conditions (7.7)-(7.10) and (7.30)-(7.32) are equivalent;

- Conditions (7.21)-(7.22) and (7.33) are equivalent.

Proof. See Proposition 4.1.

Equations (7.31)-(7.32) and (7.33) are alternative design conditions for the cluster case with respect to the work (FIORAVANTI; GONÇALVES; GEROMEL, 2013a) that 
requires the system matrices being all equal inside a given cluster, as well as (MORAIS et al., 2017), that needs the initialization with a full-order, partially mode-dependent, dynamic output feedback controller.

Furthermore, since the parametrization of $\left(A_{c \hat{\theta}(k)}, B_{c \hat{\theta}(k)}, C_{c \hat{\theta}(k)}\right)$ does not involve the transition and conditional probabilities, and besides (7.7)-(7.10) and (7.21)-(7.22) are affine with respect to $p_{i j}$ and $\alpha_{i l}$, we assume that $\mathbb{P} \in \mathbb{D}_{\mathbb{P}}$ and $\Upsilon \in \mathbb{D}_{\Upsilon}$. We define the set $\mathbb{C}_{r} \triangleq\left\{\mathcal{C}:(7.3)\right.$ for $\mathbb{P} \in \mathbb{D}_{\mathbb{P}}$ and $\Upsilon \in \mathbb{D}_{\Upsilon}$ is SS $\}$, and set

$$
p_{i j}=p_{i j}^{(h)}, \alpha_{i l}=\alpha_{i l}^{(t)},
$$

in (7.7)-(7.10) and (7.21)-(7.22) for $h \in\{1, \ldots, \eta\}, t \in\{1, \ldots, \tau\}, i \in \mathbb{N}, l \in \mathbb{M}_{i}$. We redefine $\bar{\Xi}(K)$ in this case by considering the changes described in (7.34)

$\tilde{\Xi}(K)=\{\bar{\xi}$ such that the LMls $(7.7)-(7.10)$ and $(7.21)-(7.22)$ subject to $(7.34)$ are jointly feasible\}.

Corollary 7.3. Given $K \in \mathbb{K}_{r}$ such that $\tilde{\Xi}(K) \neq \emptyset$, if $\bar{\xi} \in \tilde{\Xi}(K)$, then by setting $A_{c l}=$ $-G_{l}^{-1} R_{l}, B_{c l}=-G_{l}^{-1} F_{l}$, and $C_{c l}=K_{l}$ for all $l \in \mathbb{M}$, we get that $\mathcal{C} \in \mathbb{C}_{r},\left\|\mathcal{G}_{c}\right\|_{2}<\gamma$ and $\left\|\mathcal{G}_{c}\right\|_{\infty}<\delta$.

Example 7.4: Recalling Assumption 3.1, we set $\rho=0.5$ and use the state-feedback controller in (6.30) in the optimization problem (7.20), in order to calculate the following remaining matrices

$$
\left[\begin{array}{l|l}
A_{c i} & B_{c i}
\end{array}\right]=\left[\begin{array}{ll|l}
-0.3724 & -0.0430 & 0.8137 \\
-0.2262 & -0.4463 & 0.0024
\end{array}\right],
$$

for all $i \in \mathbb{N}$ with $r_{\sigma}(\mathcal{L})=0.8026, \gamma_{c}^{*}=6.6674$, and $\left\|\mathcal{G}_{c}\right\|_{2}=3.1058$, that is, a modeindependent dynamic output feedback controller is obtained.

On the other hand, for the $\mathcal{H}_{\infty}$ control, we set $\rho=0.5$ and use the state-feedback controller (6.31) in (7.20), in order to get following remaining matrices

$$
\left[A_{c i} \mid B_{c i}\right]=\left[\begin{array}{cc|r}
-0.1628 & -0.3275 & 0.7570 \\
-0.1937 & 0.3355 & -0.0427
\end{array}\right],
$$

for all $i \in \mathbb{N}$ with $r_{\sigma}(\mathcal{L})=0.8402, \delta=52.4994$ and $\delta_{K}=13.0697$, that is, a modeindependent $\mathcal{H}_{\infty}$ dynamic output feedback controller is obtained. 


\subsection{Final remarks}

In this chapter, we studied the design of dynamic output feedback controllers in the context of partial observation of $\hat{\theta}(k)$, for the $\mathcal{H}_{2}, \mathcal{H}_{\infty}$, and mixed $\mathcal{H}_{2} / \mathcal{H}_{\infty}$ control. Bilinear synthesis conditions were given that yields to a type of ad-hoc separation procedure that echoes the works (OLIVEIRA, 1999) and (OLIVEIRA; GEROMEL; BERNUSSOU, 2000). The flexibility of our method also allows for the robust case, in which the transition and detection probabilities are uncertain. Even though the presented formulation is an advance with respect to the current literature, the parametrization of all stabilizing dynamic controllers for the partial observation case remains an open and challenging problem. The weak point in our formulation is the choice of $K$ that can prevent the calculation of the remaining controller matrices, however some highlights can be pointed out such as retrieving the optimal controller for the suitable choice of $K$ in the mode-dependent case, and the design of cluster and mode-independent controllers. 


\section{ACADEMIC APPLICATIONS}

In this chapter, we present three academic applications in the contexts of AFTSC and NCS. In Section 8.1, we consider the control of the lateral dynamics of an unmanned aircraft subject to faults in the actuators. Two cases are studied: (1) the states are available to the controller; (2) only the output $y$ can be measured and used in the control. In Section 8.2, we consider a filtering application, by observing the states of a classical spring-mass-damper system whose measured signal are transmitted through a channel subject to data loss and noise. Finally, in Section 8.3, we present a new model for wireless NCS, unrelated to the hidden MJLS, introduced in the work (VARMA et al., 2017) and derive stability and design conditions for state-feedback controllers.

\subsection{Unmanned Aircraft}

For illustrating the results given in Chapters 4 and 7, we consider the lateraldirectional dynamics of a small unmanned aircraft in steady flight, that is illustrated in Figure 1, as modeled in (DUCARD, 2009). The states are variations in the roll rate $(\Delta p)$, the yaw rate $(\Delta r)$, the sideslip angle $(\Delta \beta)$, and the roll angle $(\Delta \phi)$, whereas the control inputs are the aileron $\left(\Delta \delta_{\text {aileron }}\right)$ and the rudder $\left(\Delta \delta_{\text {rudder }}\right)$. It is considered that the actuators are subject to abrupt faults as assumed by (COSTA; FRAGOSO; TODOROV, 2015) and illustrated by Figure 2. The transition probability matrix is given by

$$
\mathbb{P}=\left[\begin{array}{ccc}
0.6 & 0.4 & 0 \\
0.2 & 0.7 & 0.1 \\
0 & 0.9 & 0.1
\end{array}\right]
$$

and the initial probability is taken as the stationary distribution $\mu=$ $\left[\begin{array}{lll}0.3103 & 0.6207 & 0.0690\end{array}\right]$. The detection probability matrix has the following structure:

$$
\Upsilon=\left[\begin{array}{ccc}
1 & 0 & 0 \\
0 & \rho_{2} & 2-\rho_{2} \\
0 & 1-\rho_{3} & \rho_{3}
\end{array}\right]
$$


where $\rho_{i}=\operatorname{Prob}(\hat{\theta}(k)=i \mid \theta(k)=i), i \in\{2,3\}$, that is, the probability of detecting correctly the parameter $\theta(k)$ for modes 2 and 3 . Then, in the nominal mode $\theta(k)=1$, the detector would provide correct estimates of the Markov parameters, whereas for the remaining faulty states, the probability of obtaining the correct mode of operation is $\rho_{i}$. In this case, the discretized (zero-order hold, $T=0.05 \mathrm{~s}$ ) system matrices are given by

$$
\left[A_{i} \mid B_{1}\right]=\left[\begin{array}{rrrr|rr}
0.5637 & 0.1133 & -0.6607 & -0.0062 & 2.9735 & -0.0618 \\
0.0198 & 0.8368 & 1.0512 & 0.0089 & -0.1175 & 0.6414 \\
0.0033 & -0.0450 & 0.9481 & 0.0159 & 0.0112 & -0.0165 \\
0.0381 & 0.0073 & -0.0164 & 0.9999 & 0.0812 & -0.0006
\end{array}\right]
$$

for all $i \in \mathbb{N}$. Moreover the actuator failures are modeled by

$$
B_{2}=B_{1}\left[\begin{array}{ll}
0 & 0 \\
0 & 1
\end{array}\right], \quad B_{3}=-B_{2}
$$

and thus, we have that $\theta(k)=1$ is the nominal mode of operation, $\theta(k)=2$ is the mode with inactive aileron command, and $\theta(k)=3$ is the mode where the aileron is still not active and the rudder command is inverted. The exogenous input matrix is taken as, for all $i \in \mathbb{N}$,

$$
J_{i}=\left[\begin{array}{ll}
I_{4} & 0_{4 \times 2}
\end{array}\right]
$$

The objective of this example is to illustrate the state-feedback and dynamic output feedback control developed in Chapters 4 and 7, considering that $\hat{\theta}(k)$ models a simple failure detector, as done in (COSTA; FRAGOSO; TODOROV, 2015).

\subsubsection{State-feedback control}

For the $\mathcal{H}_{2}$ state-feedback control, we set

$$
C_{i}^{(2)}=\left[\begin{array}{c}
I_{4} \\
0_{2 \times 4}
\end{array}\right], \quad D_{i}^{(2)}=\left[\begin{array}{c}
0_{4 \times 2} \\
I_{2}
\end{array}\right], \quad E_{i}^{(2)}=0_{6 \times 6},
$$

for all $i \in \mathbb{N}$. We solve (4.14) by varying $\rho_{2}$ and $\rho_{3}$ in order to get Figure 6 . We note two interesting cases: (1) $\rho_{2}=\rho_{3}=1$ and $\rho_{2}=\rho_{3}=0$; (2) $\rho_{2}=1-\rho_{3}$. In the first case, we get the mode-dependent formulation, since $\operatorname{Prob}(\hat{\theta}(k)=i \mid \theta(k)=i)=1$ 
Figure $6-\gamma^{*}$ against $\rho_{2}$ and $\rho_{3}$

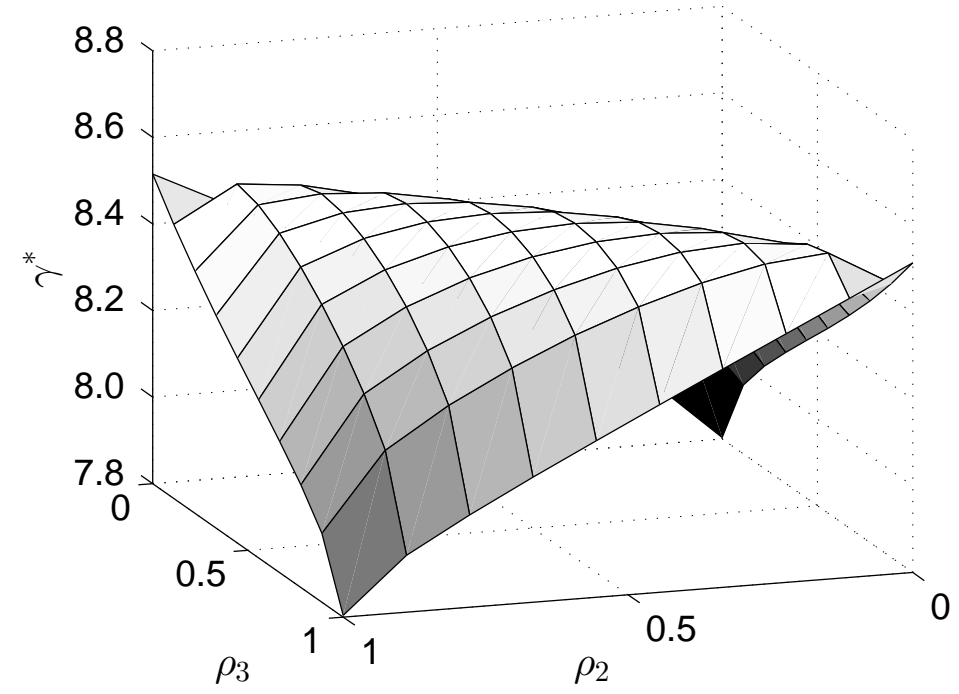

Source: Author.

for $i \in\{2,3\}$, with $\left\|\mathcal{G}_{*}\right\|_{2}=\gamma^{*}=7.8037$. Interestingly, the case characterized by $\operatorname{Prob}(\hat{\theta}(k)=i \mid \theta(k)=i)=0$ for $i \in\{2,3\}$ leads also to the mode-dependent case, since there are only two possible outcomes for $\theta(k)=2$ and $\theta(k)=3$. The controller for $\rho_{2}=\rho_{1}=3$ is shown in Equation (C.1).

On the other hand, if $\rho_{2}=1-\rho_{3}$, we get that the first two rows of (8.1) are numerically equal. This configures the cluster case for $\mathbb{N}^{1}=\{1\}$ and $\mathbb{N}^{2}=\{2,3\}$, as the Assumption 3.1 is fulfilled. Then, we get $\gamma^{*}=8.5152,\left\|\mathcal{G}_{K}\right\|_{2}=8.2633$, along with

$$
\left[\begin{array}{l}
K_{1} \\
K_{2} \\
K_{3}
\end{array}\right]=\left[\begin{array}{cccc}
-0.1874 & -0.0581 & 0.1750 & -0.4909 \\
-0.0334 & -0.6378 & -0.3274 & -0.1564 \\
\hline 0 & 0 & 0 & 0 \\
0.0002 & -0.1878 & -0.5158 & -0.1133 \\
\hline 0 & 0 & 0 & 0 \\
0.0002 & -0.1878 & -0.5158 & -0.1133
\end{array}\right] \text {, }
$$

for $\rho_{3}=0.5$, that is, the controller for $\hat{\theta}(k)=2$ and $\hat{\theta}(k)=3$ are numerically equal. Finally, we note a type of symmetry in Figure 6 with respect to $\rho_{2}$ and $\rho_{3}$, due to the structure of (8.1). 
For the $\mathcal{H}_{\infty}$ state-feedback control, we use the same values for $J_{i}, i \in \mathbb{N}$, and set

$$
C_{i}^{(\infty)}=\left[\begin{array}{cccc}
0.1 & 0 & 0 & 0 \\
0 & 1 & 0 & 0 \\
0 & 0 & 0 & 0
\end{array}\right], D_{i}^{(\infty)}=\left[\begin{array}{cc}
0 & 0 \\
0 & 0 \\
0 & 1
\end{array}\right], E_{i}^{(\infty)}=0_{3 \times 6},
$$

for all $i \in \mathbb{N}$. Considering $\rho_{2}=\rho_{3}=\rho$ in (8.1) and solving the optimization problem (4.21) by varying $\rho$, we get the guaranteed costs and actual norms shown in Figure 7. The similar remarks as made for Figure 6 can be also stated for Figure 7, that is, we

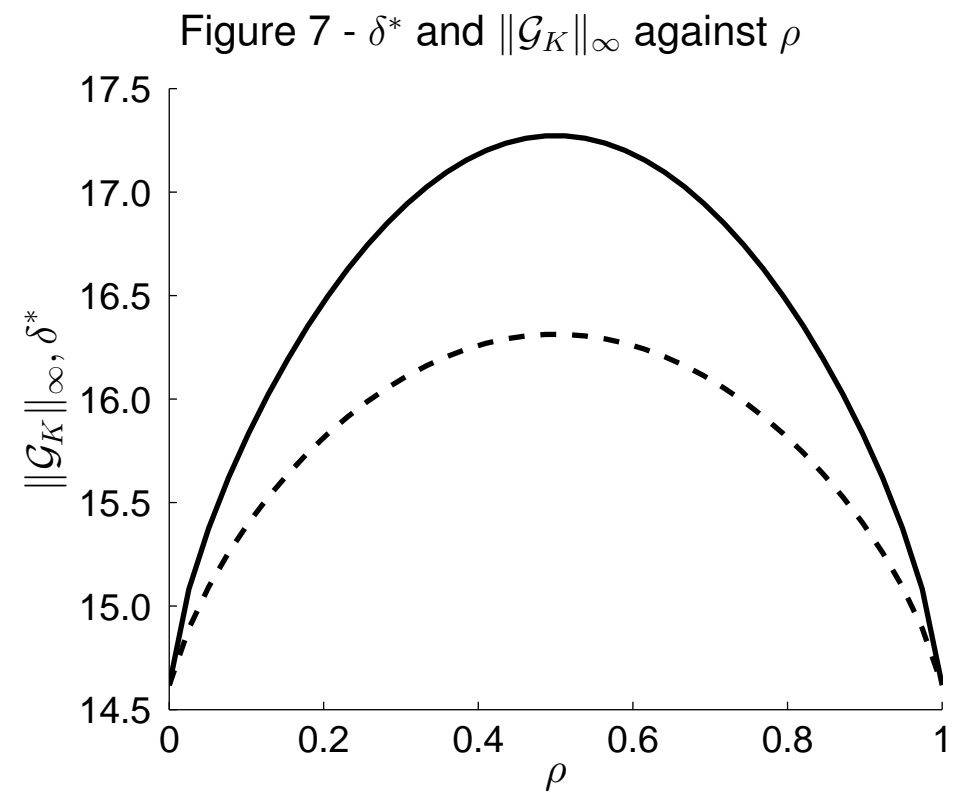

Source: Author.

get mode-dependent costs for $\rho=1$ and $\rho=0\left(\left\|\mathcal{G}_{*}\right\|_{\infty}=\delta^{*}=14.6181\right)$, as well as clusterized controllers for $\rho=0.5\left(\delta^{*}=17.2728,\left\|\mathcal{G}_{K}\right\|_{\infty}=16.3132\right)$. The controllers for both cases $\rho=1$ and $\rho=0.5$ are respectively shown in Equations (C.2) and (C.3). We recall that the symmetry displayed in the curves of Figure 7 is caused by the fact that there are only two possible "uncertain" outcomes that can be provided by the detector, namely $\hat{\theta}(k)=2$ and $\hat{\theta}(k)=3$.

We now solve (4.25) by setting $g(\gamma, \delta)=\gamma^{2}$ for three possible values for $\rho_{2}=\rho_{3}=\rho$, that is, $\rho \in\{0.5,0.7,1.0\}$. For that, we find the minimum value of $\delta$ such that (4.25) is still feasible. The values of $\gamma^{*}$ against $\delta$ are shown in Figure 8 . Figure 8 shows tradeoff curves for problem (4.25) in the sense that, as we increase the values of $\delta$, we get smaller values of $\gamma^{*}$ (and vice versa). It is intuitive also to note that the guaranteed costs for $\rho=1.0$ are smaller with respect to $\rho=0.5$, that configures the cluster case. 
Figure $8-\gamma^{*}$ against $\delta$ for $\rho=1$ (black line), $\rho=0.7$ (darker grey line), and $\rho=0.5$ (brighter grey line)

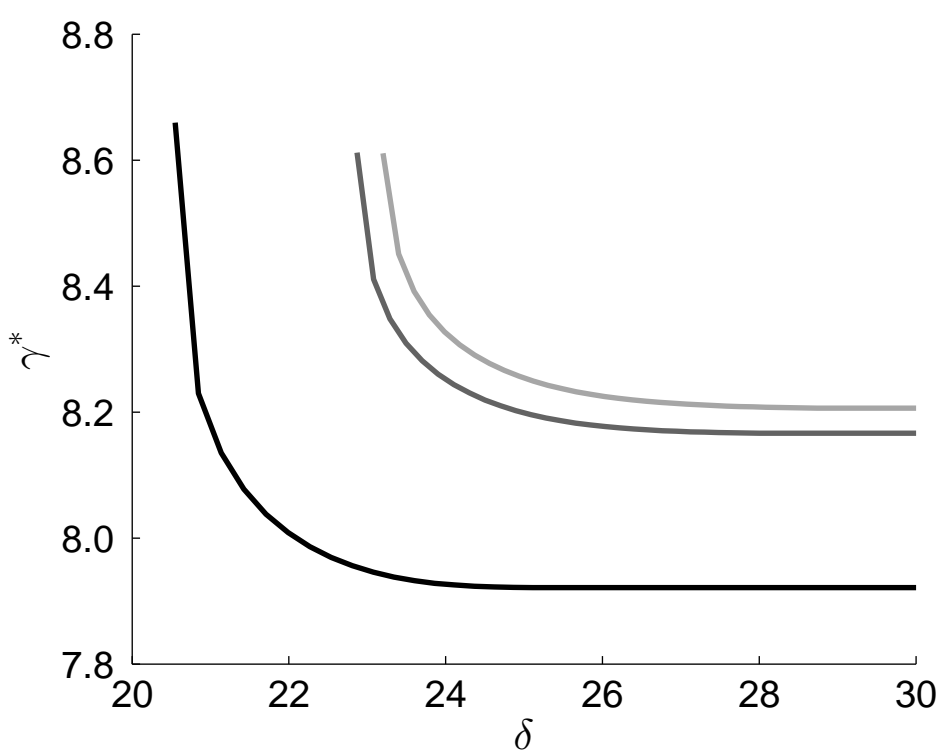

Source: Author.

We now set $\delta=23.5$ and solve (4.25) by varying $\rho \in[0,1]$. The result is shown in Figure 9. We note the similar symmetry as obtained in Figures 6 and 7, however for Figure $9-\gamma^{*}$ (black line) and $\left\|\mathcal{G}_{K}\right\|_{2}$ (dashed black line) against $\rho$

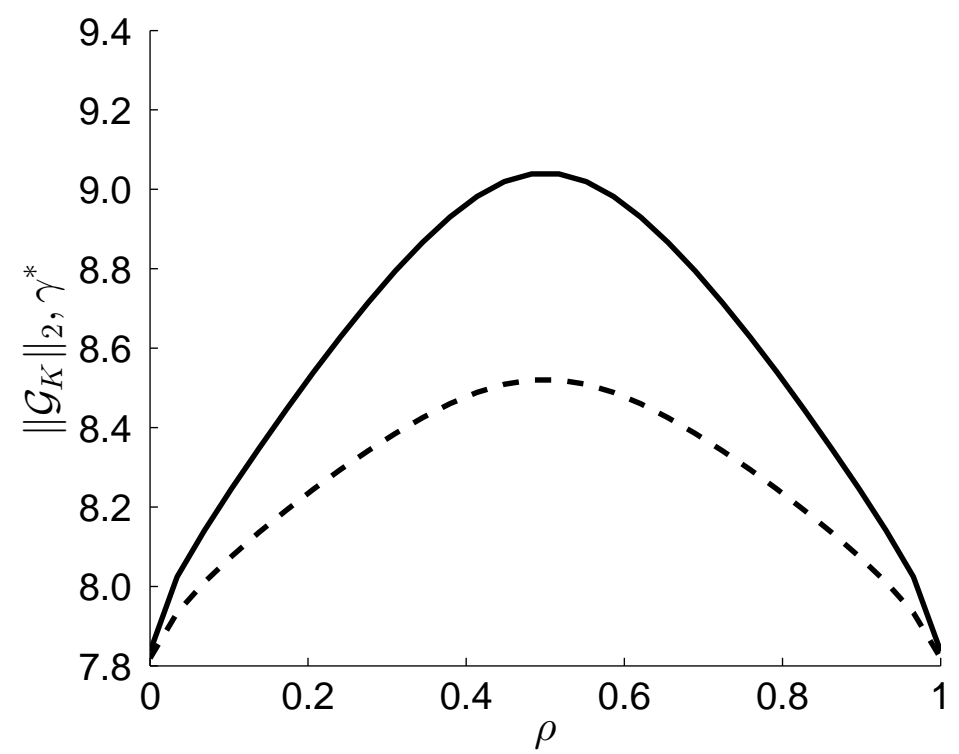

Source: Author.

$\rho=1$ and $\rho=0$, we do not obtain the optimal $\mathcal{H}_{2}$ controller, since the addition of the $\mathcal{H}_{\infty}$ control part to the problem renders the problem suboptimal $\left(\gamma^{*}=7.8360,\left\|\mathcal{G}_{K}\right\|_{2}=\right.$ 7.8192). By taking $\rho=0.7$ and $\delta=23.5$, we get the controller shown in (C.4), with $\gamma^{*}=8.7709,\left\|\mathcal{G}_{K}\right\|_{2}=8.3727$, and $\left\|\mathcal{G}_{K}\right\|_{\infty}=19.4748$. 


\section{Mixed $\mathcal{H}_{2} / \mathcal{H}_{\infty}$ state-feedback control with an uncertain $\Upsilon$}

We now consider the case in which $\Upsilon \in \mathbb{D}_{\Upsilon}$. The vertices of $\Upsilon$ are given by

$$
\left[\Upsilon_{1} \mid \Upsilon_{2}\right]=\left[\begin{array}{ccc|ccc}
1 & 0 & 0 & 1 & 0 & 0 \\
0 & 1 & 0 & 0 & \epsilon & 1-\epsilon \\
0 & 0 & 1 & 0 & 1-\epsilon & \epsilon
\end{array}\right],
$$

for $0 \leq \epsilon \leq 1$. We again set $\delta=23.5$ and solve (4.25) for $0 \leq \epsilon \leq 1$. The result is shown in Figure 10. In this example, the first thing to be pointed out is the similarity between

Figure $10-\gamma^{*}$ for $\delta=23.5$ against $\epsilon$ for the vertices of $\Upsilon$ given in (8.3)

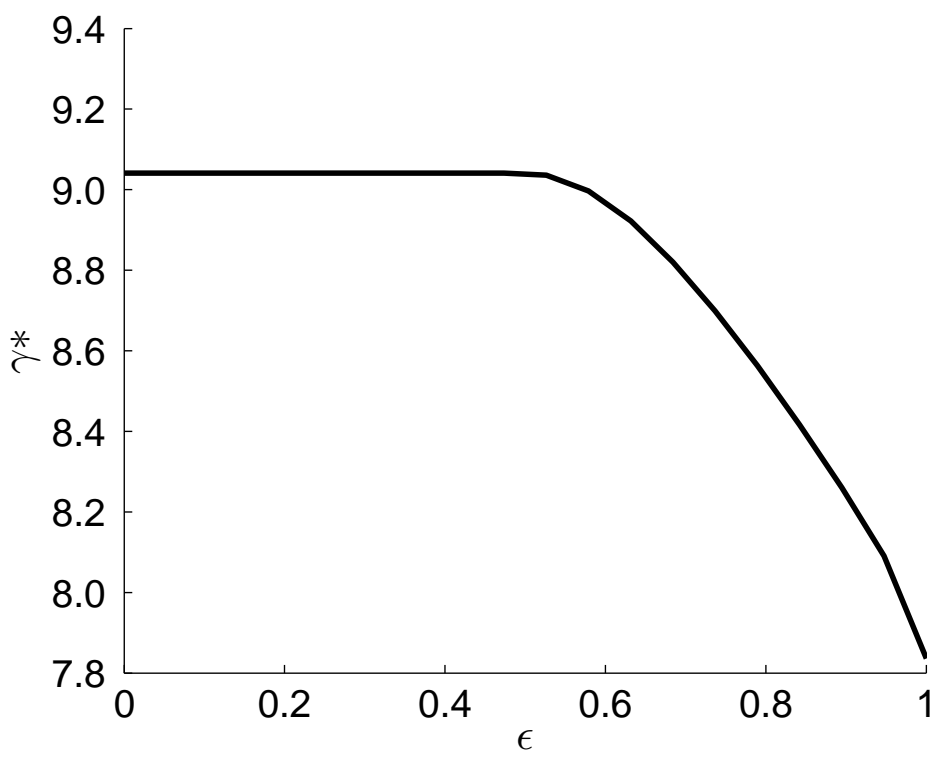

Source: Author.

Figure 9 and Figure 10 for the region defined by $\epsilon>0.5$, that is, the guaranteed costs are numerically close for both the problem with and without parametric uncertainty on $\Upsilon$. On the other hand, for $0 \leq \epsilon \leq 0.5$, the guaranteed cost remains constant (considering the numerical precision) and equal to $\gamma^{*} \approx 9.041$, that is the guaranteed cost for the cluster case. This can be explained by considering the definition of $\Upsilon$ in (3.4), and so we write that

$$
\Upsilon=b_{1} \Upsilon_{1}+b_{2} \Upsilon_{2}=\left[\begin{array}{ccc}
1 & 0 & 0 \\
0 & 1-b_{2}(1-\epsilon) & b_{2}(1-\epsilon) \\
0 & b_{2}(1-\epsilon) & 1-b_{2}(1-\epsilon)
\end{array}\right],
$$

for $b_{1}+b_{2}=1$ and $0 \leq b_{2}(1-\epsilon) \leq 1$. As explained in Example 3.2, the "clusterization" in the detector case occurs whenever some given rows in $\Upsilon$ are equal. In this case, we 
have that $1-b_{2}(1-\epsilon)=b_{2}(1-\epsilon)$ must be satisfied in order to get numerically equal second and third rows of $\Upsilon$. Thus,

$$
b_{2}=\frac{1}{2(1-\epsilon)}
$$

and then, by considering that $0 \leq b_{2} \leq 1$, we have that the range of $\epsilon$ in which the previous equation is valid is $0 \leq \epsilon \leq 0.5$. Recalling that the class of problems of parametric uncertainty always yields the worst-case scenario, we have that for $0 \leq \epsilon \leq$ 0.5 , the guaranteed cost will always converge to the worst cost obtained by (4.25) in this situation, that is, the cluster case cost. On the other hand, for $\epsilon>0.5$, the value of $b_{2}$ would remain "fixed" in $b_{2}=1$, then $\Upsilon_{2}$ becomes dominant in the optimization problem, yielding cost values that are numerically close to the ones presented in Figure 10.

\subsubsection{Dynamic output feedback control}

We now consider that $x$ is not available to the control anymore, but only a measured output $y$ subject to noise. In this case, we set the remaining matrices of system (7.1) as follows

$$
L_{i}=\left[\begin{array}{llll}
0 & 0 & 1 & 0 \\
0 & 0 & 0 & 1
\end{array}\right], H_{i}=\left[\begin{array}{ll}
0_{2 \times 4} & I_{2}
\end{array}\right],
$$

for all $i \in \mathbb{N}$, that is, we consider that we can measure the variations on the sideslip and roll angles. For the mode-dependent case, we set $\rho=1.0$ and use the optimal $\mathcal{H}_{2}$ state-feedback control (C.1) in (7.20) to obtain the remaining controller matrices shown in (C.5) and (C.6), with $\gamma^{*}=\left\|\mathcal{G}_{*}\right\|_{2}=8.2335$. We note that (i) $K$ and $B_{c}$ coincide with the gains obtained through the control and filtering CARE in (B.3) and (B.4), as expected; (ii) the observer gains $B_{c i}$ in (C.6) are numerically equal for $i \in \mathbb{N}$, since there is no variation in $(A, B, J, H)$.

We now solve (4.14) to obtain the $\mathcal{H}_{2}$ state-feedback controller, use it as an input in (7.20) to investigate the behavior of $\gamma^{*}$ and $\left\|\mathcal{G}_{c}\right\|_{2}$ with respect to $\rho \in[0,1]$. The result is shown in Figure 11. In this example, we note the similar symmetry of Figure 11 compared to Figures 6 and 7, and more importantly, the optimal $\mathcal{H}_{2}$ dynamic output feedback controller can be obtained by setting $\rho=0$ and $\rho=1$, along with clusterized dynamic controllers for $\rho=0.5$. For the cluster case $(\rho=0.5)$, the controller is given by 
Figure $11-\gamma^{*}$ (black line) and $\left\|\mathcal{G}_{c}\right\|_{2}$ (dashed black line) against $\rho$

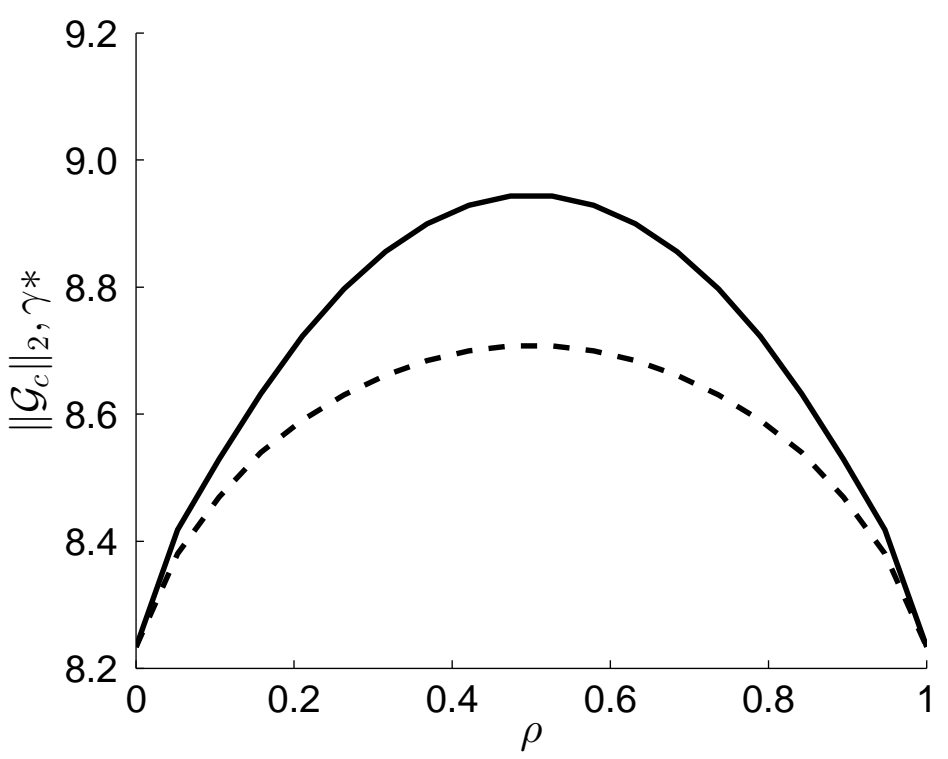

Source: Author.

(8.2), along with

$$
\left[\begin{array}{c}
A_{c 1} \\
A_{c i}
\end{array}\right]=\left[\begin{array}{rrrr}
0.0121 & -0.0251 & 0.4127 & -1.6416 \\
0.0210 & 0.4315 & 0.1302 & -0.1000 \\
0.0016 & -0.0338 & 0.4018 & 0.0273 \\
0.0203 & 0.0084 & -0.0693 & 0.4430 \\
\hline 0.5150 & 0.0141 & -0.5141 & -0.1855 \\
0.0502 & 0.7511 & 0.3197 & 0.0364 \\
-0.0055 & 0.0471 & 0.5637 & 0.0599 \\
0.0413 & 0.0096 & -0.0103 & 0.3941
\end{array}\right]
$$

for $i \in \mathbb{N}^{2}=\{2,3\}$, along with

$$
\left[B_{c 1} \mid B_{c i}\right]=\left[\begin{array}{rr|rr}
-0.4322 & 0.3141 & -0.2348 & 0.1307 \\
0.7413 & 0.0860 & 0.4892 & -0.0724 \\
0.5319 & -0.0195 & 0.3270 & -0.0305 \\
-0.0367 & 0.4224 & 0.0044 & 0.6086
\end{array}\right]
$$

for $i \in \mathbb{N}^{2}$, with $\gamma^{*}=8.9450$ and $\left\|\mathcal{G}_{c}\right\|_{2}=8.7084$.

We now focus our efforts on the $\mathcal{H}_{\infty}$ control and proceed with the steps of Algorithm 4 by solving (4.21) and (7.28) for $\rho \in[0,1]$. The result is shown in Figure 12. Contrarily as it happened in Example 7.2, we are able to approximate the optimal $\mathcal{H}_{\infty}$ dynamic 
Figure $12-\delta^{*}$ (black line) and $\left\|\mathcal{G}_{c}\right\|_{\infty}$ (dashed black line) against $\rho$

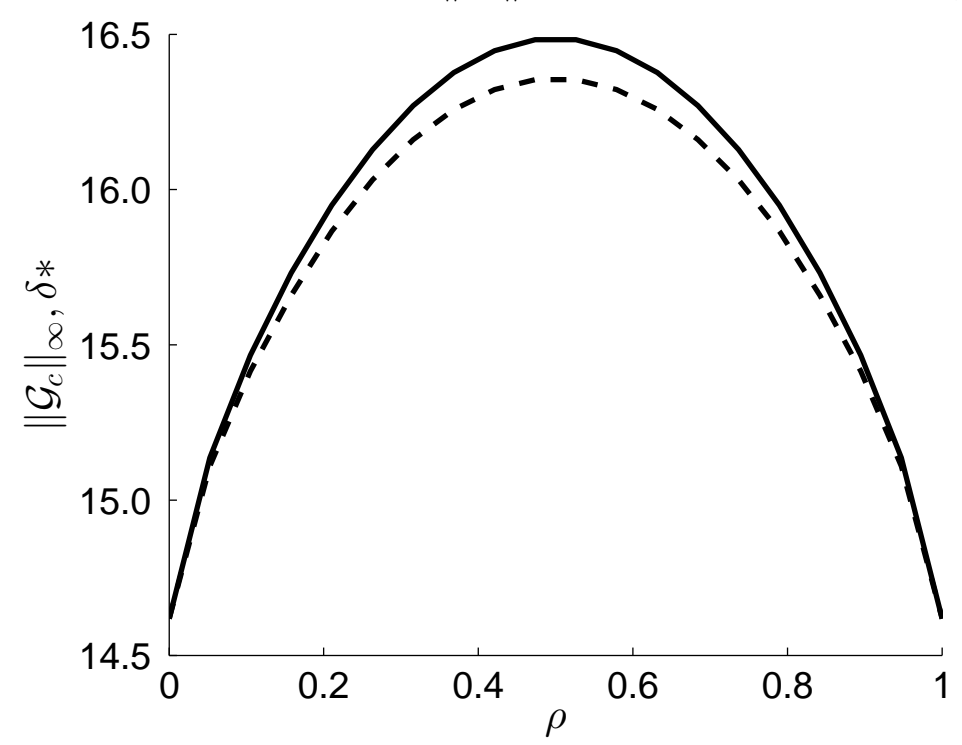

Source: Author.

output feedback control by feeding to (7.28) the state-feedback gains (C.2) obtained from (4.21) with $\rho=0$ and $\rho=1$. In this case, by setting $\rho=1$, we get the controller matrices shown in (C.7) and (C.8), with $\delta^{*}=\left\|\mathcal{G}_{*}\right\|_{\infty}=14.6181$. Additionally, for $\rho=$ 0.5 , we use the state-feedback controller (C.3) in (7.28) in order to get the remaining controller matrices,

$$
\left[\begin{array}{c}
A_{c 1} \\
\hline A_{c i}
\end{array}\right]=\left[\begin{array}{rrrr}
-0.0829 & 1.8097 & -16.2013 & -3.3292 \\
0.0246 & 0.3613 & 2.6831 & 0.4537 \\
0.0017 & -0.0299 & 0.0330 & 0.0146 \\
0.0205 & 0.0533 & -0.3181 & -0.0246 \\
\hline 0.5635 & 0.1369 & 1.7833 & -6.6703 \\
0.0202 & 0.8628 & 1.4207 & -0.8901 \\
0.0076 & -0.0709 & -0.0910 & -0.0349 \\
0.0392 & 0.0042 & 0.1157 & -0.4600
\end{array}\right],
$$

for $i \in \mathbb{N}^{2}=\{2,3\}$, along with

$$
\left[B_{c 1} \mid B_{c i}\right]=\left[\begin{array}{rr|rr}
-2.9078 & -1.4961 & -2.4374 & 6.6545 \\
-1.1954 & -0.3019 & -0.7264 & 0.8968 \\
0.9747 & -0.0127 & 1.3067 & 0.0884 \\
-0.2055 & 0.8964 & -0.1053 & 1.4716
\end{array}\right]
$$

for $i \in \mathbb{N}^{2}$, with $\delta^{*}=16.4870$ and $\left\|\mathcal{G}_{c}\right\|_{\infty}=16.3580$. 
Finally, we design a mixed $\mathcal{H}_{2} / \mathcal{H}_{\infty}$ dynamic output feedback controller for $\rho=0.7$. For that, we solve (4.14) and get the state-feedback controller gains shown in (C.9). By setting $g(\gamma, \delta)=\gamma^{2}$ and $\delta=25$ in (7.29) and using (C.9) as an input, we get the remaining controller matrices, with $\gamma^{*}=8.8401,\left\|\mathcal{G}_{c}\right\|_{2}=8.6528$, and $\left\|\mathcal{G}_{c}\right\|_{\infty}=21.2648$.

\subsection{Mass-spring-damper system}

The second example is a modified version of the one given in (FIORAVANTI; GONÇALVES; GEROMEL, 2015), consisting of a classical two mass systems coupled by a spring and a damper, where the first mass is also connected to a wall by a spring. The first state is the position of the first mass, the second state, the position of the second mass, and the third and fourth states represent the velocities of the first and second mass, respectively. The states are transmitted through a channel subject to varying noise levels and data loss, modeled by a Markov chain. There are three states: (1) the transmission is successful with a nominal noise level; (2) the transmission is successful with an augmented noise level; (3) the transmission is lost. We model this process as a Markov chain with state space $\mathbb{N}=\{1,2,3\}$ and

$$
\mathbb{P}=\left[\begin{array}{lll}
0.7 & 0.2 & 0.1 \\
0.5 & 0.4 & 0.1 \\
0.5 & 0.1 & 0.4
\end{array}\right],
$$

and take the initial distribution as the stationary, that is, $\mu=\left[\begin{array}{lll}0.6250 & 0.2321 & 0.1429\end{array}\right]$. The system is discretized via a zero order hold of period $T=0.5 \mathrm{~s}$ in each input, as in (FIORAVANTI; GONÇALVES; GEROMEL, 2015), yielding the following system matrices:

$$
\left[A_{i} \mid J_{i}\right]=\left[\begin{array}{rrrr|rr}
-0.7562 & 0.5086 & 0.0791 & 0.1435 & 0.0974 & 0 \\
0.2092 & 0.5604 & 0.0718 & 0.4012 & 0.0559 & 0 \\
-1.6559 & 0.0736 & -0.7588 & 0.5113 & -1.7562 & 0 \\
-0.1463 & -1.2887 & 0.2556 & 0.5140 & 0.2092 & 0
\end{array}\right]
$$


and the sensor matrices are given by

$$
\left[\begin{array}{c|c}
L_{1} & H_{1} \\
\hline L_{2} & H_{2} \\
\hline L_{3} & H_{3}
\end{array}\right]=\left[\begin{array}{cccc|cc}
0 & 0 & 1.0 & 0 & 0 & 0.1 \\
\hline 0 & 0 & 0.1 & 0 & 0 & 1.0 \\
\hline 0 & 0 & 0 & 0 & 0 & 0
\end{array}\right] .
$$

We want to estimate the position of the second mass $x_{2}$ for the $\mathcal{H}_{2}$ filtering and the position of the first mass $x_{1}$ for the $\mathcal{H}_{\infty}$ filtering:

$$
\left[\begin{array}{c|c}
C_{i}^{(2)} & E_{i}^{(2)} \\
\hline C_{i}^{(\infty)} & D_{i}^{(\infty)}
\end{array}\right]=\left[\begin{array}{llll|ll}
0 & 1 & 0 & 0 & 0 & 0 \\
\hline 1 & 0 & 0 & 0 & 0 & 0
\end{array}\right] .
$$

The detection/asynchronous behavior is modeled as follows

$$
\Upsilon=\left[\begin{array}{ccc}
\rho & 1-\rho & 0 \\
1-\rho & \rho & 0 \\
0 & 0 & 1
\end{array}\right]
$$

with $\rho \in[0,1]$, that is, the modes of operation that are subject to noise cannot be perfectly distinguished, but only the case in which the data is lost.

We calculate the $\mathcal{H}_{2}$ and $\mathcal{H}_{\infty}$ filters by solving (5.16) and (5.22) for $\rho \in$ $\{0,0.3,0.5,0.7,1.0\}$. The values $\gamma^{*},\left\|\mathcal{G}_{f}\right\|$, and $\delta^{*}$ and $\left\|\mathcal{G}_{f}\right\|_{\infty}$ are shown in Table 1. We note a similar type of symmetry in Table 1 with respect to the guaranteed costs and

Table 1 - Values of $\gamma^{*},\left\|\mathcal{G}_{f}\right\|_{2}, \delta^{*}$, and $\left\|\mathcal{G}_{f}\right\|_{\infty}$ in function of $\rho$.

\begin{tabular}{|c|c|c|c|c|}
\hline \hline$\rho$ & $\gamma^{*}$ & $\left\|\mathcal{G}_{f}\right\|_{2}$ & $\delta^{*}$ & $\left\|\mathcal{G}_{f}\right\|_{\infty}$ \\
\hline \hline 1.0000 & 0.2405 & 0.2405 & 0.5038 & 0.5038 \\
\hline 0.7000 & 0.3380 & 0.3258 & 0.7522 & 0.7427 \\
\hline 0.5000 & 0.3448 & 0.3373 & 0.8650 & 0.8318 \\
\hline 0.3000 & 0.3380 & 0.3258 & 0.7522 & 0.7427 \\
\hline 0 & 0.2405 & 0.2405 & 0.5038 & 0.5038 \\
\hline
\end{tabular}

norms compared with the previous example, due to the conditional matrix structure in (8.4). For $\rho=1$ and $\rho=0$, we get the mode-dependent cost, and thus the optimal $\mathcal{H}_{2}$ and $\mathcal{H}_{\infty}$ filters are obtained. For $\rho=0.7$ and $\rho=0.3$, we get the same values of guaranteed costs and actual system norms, and for $\rho=0.5$, a clusterization is induced in the states $\mathbb{N}^{1}=\{1,2\}$ and $\mathbb{N}^{2}=\{3\}$, where the $\mathcal{H}_{\infty}$ filter matrices are transcribed 
below

$$
\left[\begin{array}{c|c}
A_{f i} & B_{f i} \\
\hline C_{f i} & D_{f i}
\end{array}\right]=\left[\begin{array}{rrrr|r}
-0.7141 & 0.5049 & 0.0508 & 0.1357 & 0.0378 \\
0.2245 & 0.5579 & 0.0761 & 0.3980 & 0.0005 \\
-2.3441 & 0.1539 & -0.4122 & 0.6455 & -0.5126 \\
-0.0392 & -1.2992 & 0.1564 & 0.4927 & 0.1205 \\
\hline 0.9960 & 0.0012 & 0.0092 & 0.0014 & -0.0094
\end{array}\right]
$$

for $i \in \mathbb{N}^{1}$, and

$$
\left[\begin{array}{c|c}
A_{f 3} & B_{f 3} \\
\hline C_{f 3} & D_{f 3}
\end{array}\right]=\left[\begin{array}{cccc|c}
-0.7401 & 0.5061 & 0.0858 & 0.1408 & 0 \\
0.2184 & 0.5590 & 0.0756 & 0.3997 & 0 \\
-1.9449 & 0.1192 & -0.8793 & 0.5599 & 0 \\
-0.1118 & -1.2942 & 0.2700 & 0.5082 & 0 \\
\hline 1 & 0 & 0 & 0 & 0
\end{array}\right] .
$$

The guaranteed costs and actual norms in Table 1 are numerically equal to the ones calculated with the conditions in (FIORAVANTI; GONÇALVES; GEROMEL, 2008; GONÇALVES; FIORAVANTI; GEROMEL, 2009; GONÇALVES; FIORAVANTI; GEROMEL, 2010) for the mode-dependent and cluster cases.

We now investigate the mixed $\mathcal{H}_{2} / \mathcal{H}_{\infty}$ filtering for $\rho \in\{0,0.3,0.5,0.7,1.0\}$. We set $g(\gamma, \delta)=\delta$ and $\gamma=0.5$ and calculate the filters through (5.23). The resulting guaranteed cost and actual system norms are shown in Table 2. The behavior of

Table 2 - Values of $\delta^{*},\left\|\mathcal{G}_{f}\right\|_{\infty}$, and $\left\|\mathcal{G}_{f}\right\|_{2}$ in function of $\rho$.

\begin{tabular}{|c|c|c|c|}
\hline \hline$\rho$ & $\delta^{*}$ & $\left\|\mathcal{G}_{f}\right\|_{\infty}$ & $\left\|\mathcal{G}_{f}\right\|_{2}$ \\
\hline \hline 1.0000 & 0.9538 & 0.8401 & 0.4214 \\
\hline 0.7000 & 1.1250 & 1.0378 & 0.4114 \\
\hline 0.5000 & 1.1671 & 1.0845 & 0.4005 \\
\hline 0.3000 & 1.1250 & 1.0378 & 0.4114 \\
\hline 0 & 0.9538 & 0.8401 & 0.4214 \\
\hline
\end{tabular}

the costs is similar to the ones presented in Table 1, where the difference lies in the conservatism associated with the mode-dependent case due to the $\mathcal{H}_{2}$ aspect of the problem. The filter matrices for $\rho=0.7$ are shown in Equation C.10.

We now investigate the error dynamic by injecting a white noise (covariance ma- 
trix equals to identity) input in system (5.3). Figure 13 shows the mean value of $\left\|e_{2}(k)\right\|^{2}$ and standard deviation for a Monte Carlo simulation of 1000 rounds, as well as $\left\|\mathcal{G}_{f}\right\|_{2}^{2}=0.1693$. The Markov chain is simulated following the procedure of Chapter 15 of (TRANTER et al., 2003). We note that, by the choice of the initial probability con-

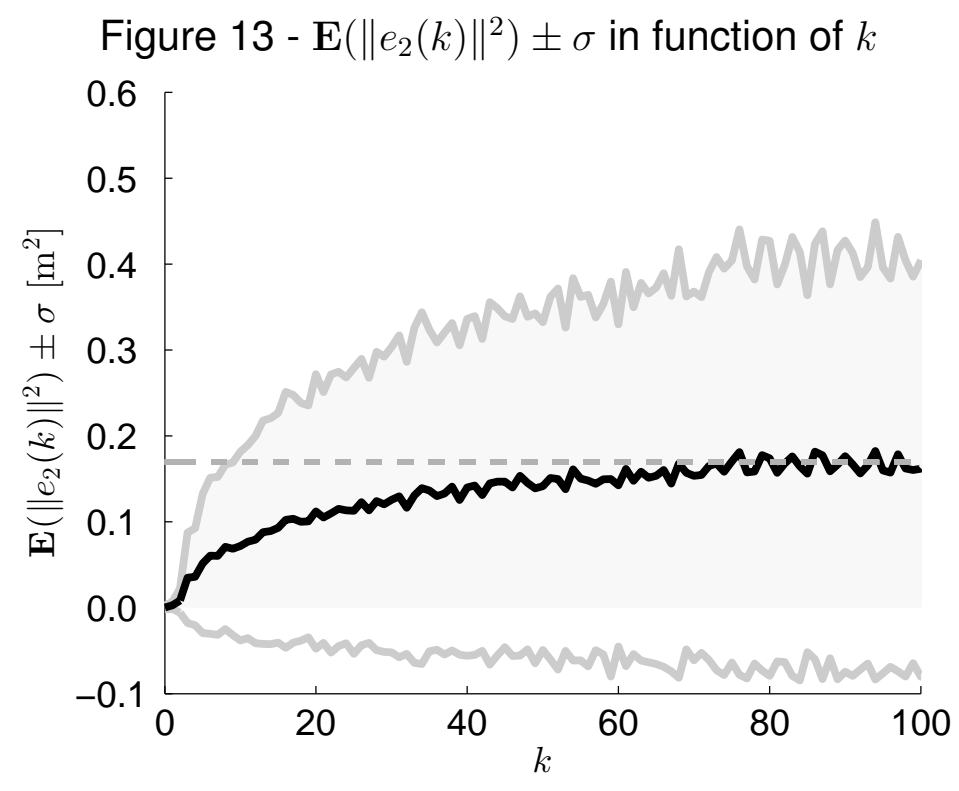

Source: Author.

dition $\mu$ as the stationary distribution, we have that $\left\|\mathcal{G}_{f}\right\|_{2}^{2}=\lim _{k \rightarrow \infty} \mathbf{E}\left(\left\|e_{2}(k)\right\|^{2}\right)$ (see (3.15)). This can be perceived in Figure 13 , as the value of $\mathbf{E}\left(\left\|e_{2}(k)\right\|^{2}\right)$ slowly tends to $\left\|\mathcal{G}_{f}\right\|_{2}^{2}$.

Let us focus now on the Bernoulli filters and set

$$
\mathbb{P}=\left[\begin{array}{lll}
0.6250 & 0.2321 & 0.1429 \\
0.6250 & 0.2321 & 0.1429 \\
0.6250 & 0.2321 & 0.1429
\end{array}\right]
$$

We calculate (5.37) and (5.44) $\mathcal{H}_{2}$ and $\mathcal{H}_{\infty}$ filters by setting $\rho=0.7$ in order to get $\left\|\mathcal{G}_{*}\right\|_{2}=\gamma^{*}=0.2738$ and $\left\|\mathcal{G}_{*}\right\|_{\infty}=0.5327$. The filter structures are shown in (C.11) and (C.12). We point out the interesting behavior of the $\mathcal{H}_{2}$ filter for the mode $\theta(k)=3$, that considers a data loss. In this mode, the filter dynamic matrix $A_{f 3}$ in (C.11) and the system matrix $A_{i}$ are numerically close, that is, the filter tries to replicate the system's dynamics in the case there is no input. Curiously, this is not the case for the $\mathcal{H}_{\infty}$ filter in (C.12). Finally, for illustrating the behavior of the Bernoulli $\mathcal{H}_{\infty}$ filter, we apply the 
following exogenous input to (5.3),

$$
\begin{aligned}
& w_{1}(k)=\left\{\begin{aligned}
0.875 v_{1}(k) \sin (k), & 0 \leq k \leq 50, \\
0, & k>0,
\end{aligned}\right. \\
& w_{2}(k)=\left\{\begin{aligned}
v_{2}(k), & 0 \leq k \leq 50, \\
0, & k>0,
\end{aligned}\right.
\end{aligned}
$$

where $v_{1}(k) \sim \mathcal{U}(0,0.1)$ and $v_{2}(k) \sim \mathcal{N}(0,1 e-6)$. We plot the mean values of $z_{f}(k)$ and $x_{1}(k)$ for a Monte Carlo simulation of 1000 rounds in Figure 14. The standard deviations are omitted for clarity of presentation. We note that the filter is able to follow the state

Figure 14 - $\mathbf{E}\left(x_{1}(k)\right)$ (black line) and $\mathbf{E}\left(z_{f}(k)\right)$ (grey line) against $k$ for the $\mathcal{H}_{\infty}$ filter

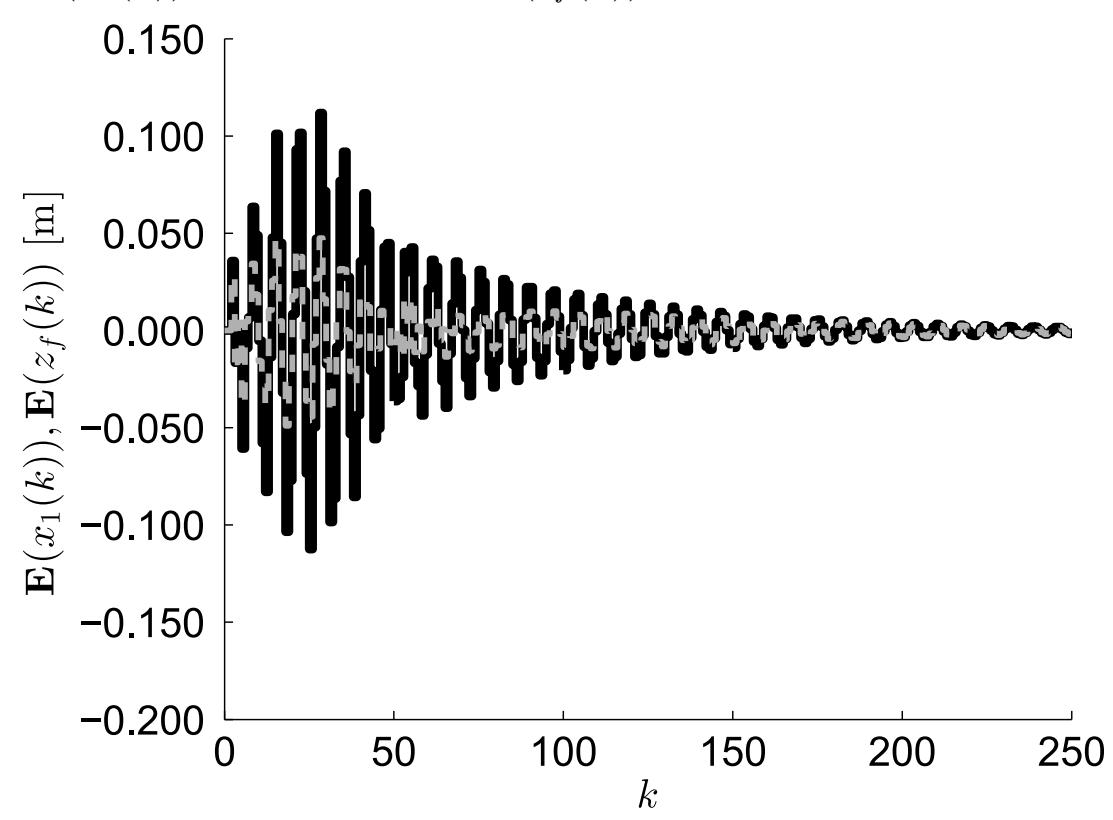

Source: Author.

$x_{1}$ for most of the time. In this case, we get

$$
\sqrt{\frac{\sum_{k=0}^{1000} \mathbf{E}\left(\left\|e_{\infty}(k)\right\|^{2}\right)}{\sum_{k=0}^{1000} \mathbf{E}\left(\|w(k)\|^{2}\right)}} \approx 0.1888
$$

that is less than $\left\|\mathcal{G}_{f}\right\|_{\infty}=0.5327$, as expected.

\subsection{Stochastic Allowable Transmission Intervals - The $(\eta, \delta)$-SATI}

We now consider a different problem than the one that was studied in the last chapters, that is, we do not use the hidden MJLS framework in this application. Instead, the aim is to find mean-square stability conditions for a class of MJLS in the context 
of WNCS applications, and consequently presenting LMls for the synthesis of modeindependent controllers ${ }^{1}$. The use of wireless networks in control systems offers many advantages in terms of ease of implementation, maintenance, flexibility, and reduced costs. However, it also induces inevitable communication imperfections and constraints such as time-varying sampling, packet drops, scheduling, delays, limited bandwidth, which may deteriorate the performance of the closed-loop system. This has motivated many researchers over the last decades to analyse and design suitable control strategies for WNCS, see, e.g., (HEEMELS; WOUW, 2010; HESPANHA; NAGHSHTABRIZI; $\mathrm{XU}, 2007)$ and the references therein. Figure 15 illustrates the schematic of a WNCS.

Figure 15 - Schematic of the WNCS

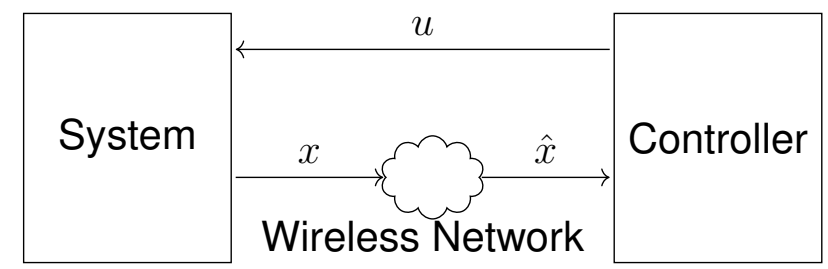

Source: Taken from (OLIVEIRA et al., 2018), adapted from (VARMA et al., 2017).

An important parameter in NCS is the maximum allowable transmission interval (MATI), that is a bound on the maximum interval between two successive transmissions that guarantees stability of the closed-loop system. Various works provide methodologies to compute bounds on the MATI to ensure stability, see, for instance, (NESIC; TEEL, 2004; CARNEVALE; TEEL; NESIC, 2007; DONKERS et al., 2011; POSTOYAN; NEŠIĆ, 2016). However, wireless networks are usually stochastic due to external factors such as channel fading, shadowing, potential collisions etc, that leads to uncertain transmissions. In this sense, the work (VARMA et al., 2017) introduced a concept for analysing the stability of WNCS called Stochastic Allowable Transmission Intervals, the SATI. The objective is twofold: (1) providing an alternative to the MATI for stochastic networks, see, for instance, and references therein, that is mainly suitable for deterministic networks; (2) establishing a framework for energy efficiency in WNCS. Specifically in (VARMA et al., 2017), the adopted strategy is emulation, that is, a asymptoticaly stabilizing controller is given without considering the network effects, that are computed afterwards. In this section, we focus on the design of state-feedback controllers for the SATI. Our results can also be found in (OLIVEIRA et al., 2018).

\footnotetext{
${ }^{1}$ This application is a collaboration with the Centre de Recherche en Automatique de Nancy (CRAN).
} 
The idea of MATI is allowing the network to transmit with lower resources in a given interval of length $N_{s}$, with a cumulative probability $\eta$. After $N_{s}$, in order to ensure the stability of the closed-loop system, the network must use all the available power resources inducing the maximum probability of successful transmission $\delta$. It is clear that for implementation in real WNCS, a simple acknowledgement scheme is necessary to guarantee that the transmission probabilities are being correctly set, see, for instance, (VARMA et al., 2017). In this context, we define the clock $\tau(k) \in\{1,2, \ldots\}$ with the following dynamic

$$
\tau(k+1)= \begin{cases}1, & \text { successful communication at } k \\ \tau(k)+1, & \text { failed communication at } k .\end{cases}
$$

The probabilities of packet drop-out $e_{\tau(k)} \in[0, \delta]$ for $1 \leq \tau(k) \leq N_{s}$ are considered to be unknown and fixed, but must respect the cumulative probability of transmitting inside the interval defined by $N_{s}$,

$$
\eta \triangleq 1-\prod_{i=1}^{N_{s}} e_{i}
$$

After $\tau(k)>N_{s}$, the network tries to transmit with the maximum probability $\delta$. Then, the SATI working scheme is illustrated in Figure 16. The idea in (VARMA et al., 2017)

Figure 16 - The SATI working scheme

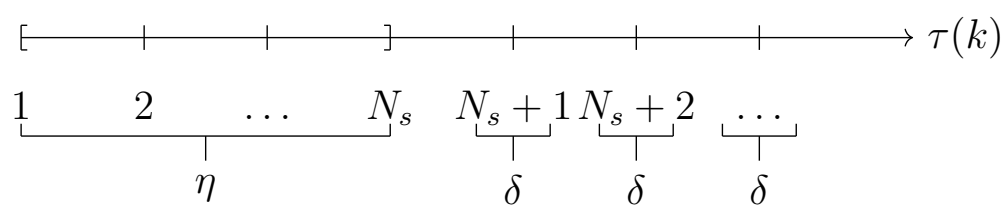

Source: Author.

is to model the clock as a Markov chain with $N=N_{s}+1$ states. For $1 \leq \tau(k) \leq N_{s}$, the transition probabilities follow the error probabilities $e_{i}$ depending on the clock value. For $\tau(k)>N_{s}$, since the network uses a fixed transmission probability, all the possible clock values are encompassed by the virtual state $N_{s}+1$. Formally, we have that

$$
\theta(k)= \begin{cases}\tau(k), & \tau(k) \leq N_{s}, \\ N_{s}+1, & \tau(k)>N_{s},\end{cases}
$$

and set the state-space of $\theta(k)$ as $\mathbb{N} \triangleq\left\{1,2, \ldots, N_{s}+1\right\}$. The resulting graph for this Markov chain is shown in Figure 17. The transition probability matrix in this case is 


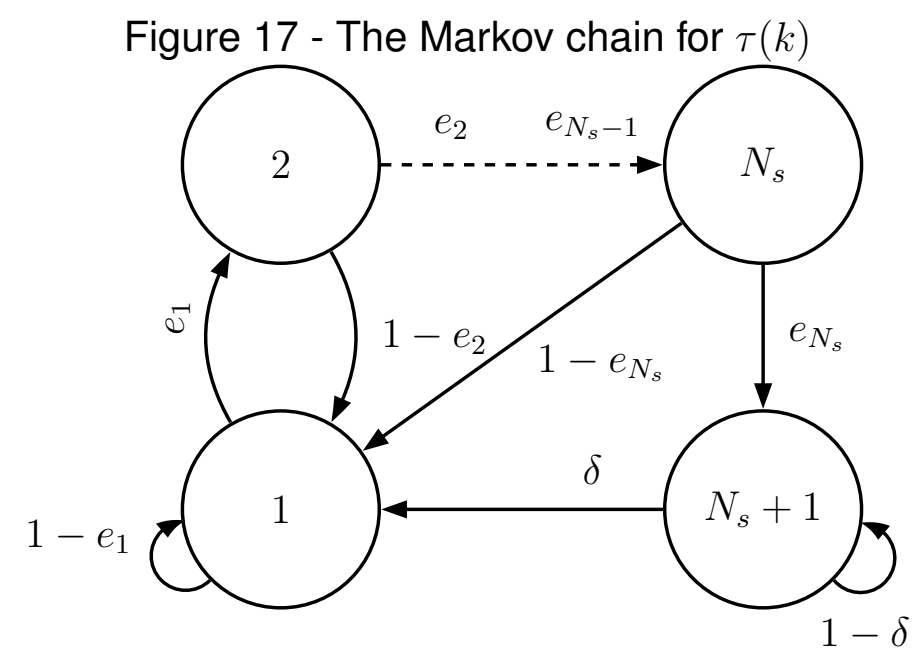

Source: Taken from (OLIVEIRA et al., 2018), adapted from (VARMA et al., 2017).

given by

$$
\mathbb{P} \triangleq\left[\begin{array}{ccccc}
1-e_{1} & e_{1} & 0 & \ldots & 0 \\
1-e_{2} & 0 & e_{2} & \ldots & 0 \\
\vdots & & & & \\
1-e_{N_{s}} & 0 & 0 & \ldots & e_{N_{s}} \\
\delta & 0 & 0 & \ldots & 1-\delta
\end{array}\right]
$$

For modeling the dynamics of the closed-loop system, we consider the system without the network effects as follows,

$$
\begin{aligned}
x(k+1) & =A_{p} x(k)+B_{p} u(k) \\
x(0) & =x_{0},
\end{aligned}
$$

with $x(k) \in \mathbb{R}^{n}$ and $u(k) \in \mathbb{R}^{m}$. Since the WNCS is subject to packet drop-outs, we define the networked version of $x(k)$ as follows,

$$
\hat{x}(k)= \begin{cases}x(k), & \text { successful transmission, } \\ \hat{x}(k-1), & \text { otherwise, }\end{cases}
$$

that is, a zero-order hold strategy. Consequently, we want to design the following control law,

$$
u(k)=K \hat{x}(k) .
$$


Then, we can write the closed-loop system as a MJLS as follows

$$
\begin{aligned}
\tilde{x}(k+1) & =A_{1+1_{\theta(k)>1}} \tilde{x}(k) \\
\tilde{x}(0) & =\tilde{x}_{0}, \theta(0)=\theta_{0},
\end{aligned}
$$

for arbitrary $\hat{x}(-1) \in \mathbb{R}^{n}$, where $\tilde{x}(k) \triangleq\left[\begin{array}{ll}x(k)^{\prime} & \hat{x}(k-1)^{\prime}\end{array}\right]^{\prime}$, along with

$$
A_{1} \triangleq\left[\begin{array}{cc}
A_{p}+B_{p} K & 0 \\
I_{n} & 0
\end{array}\right], A_{2} \triangleq\left[\begin{array}{cc}
A_{p} & B_{p} K \\
0 & I_{n}
\end{array}\right] .
$$

The goal is to design $K$ that stochastically stabilizes (8.12) for given $\left(N_{s}, \eta, \delta\right)$ and all possible values of $e_{i}$ in (8.9) subject to (8.8). For that, we define the set of admissible controllers

$$
\mathbb{K}\left(N_{s}, \eta, \delta\right) \triangleq\left\{K \in \mathbb{B}\left(\mathbb{R}^{n}, \mathbb{R}^{m}\right):(8.12) \text { is SS for all } e_{i} \text { subject to (8.8) }\right\}
$$

We need the following auxiliary result.

Lemma 8.1. We have that $\eta=\sum_{i=1}^{N_{s}}\left(1-e_{i}\right) \prod_{j=1}^{i-1} e_{j}$.

Proof. Define $g_{m} \triangleq 1-\sum_{i=m}^{N_{s}}\left(1-e_{i}\right) \prod_{j=m}^{i-1} e_{j}$, for $m \in\left\{1, \ldots, N_{s}\right\}$. For $m=N_{s}$, $g_{N_{s}}=e_{N_{s}}$ (recalling that $\prod_{j=N_{s}}^{N_{s}-1} e_{j}=1$ ) and $g_{m}=e_{m} g_{m+1}$, for $m \in\left\{1, \ldots, N_{s}-1\right\}$. By taking $m=1$ and considering the previous recursive formula, we can write that $g_{1}=e_{1} \prod_{i=2}^{N_{s}} e_{i}=\prod_{i=1}^{N_{s}} e_{i}=1-\eta$, where the last equality is obtained from (8.8), thus $\eta=1-g_{1}=\sum_{i=1}^{N_{s}}\left(1-e_{i}\right) \prod_{j=1}^{i-1} e_{j}$.

For a given $K$, we have the following theorem.

Theorem 8.1. Given the controller gain $K, N_{s}, \eta \in[0,1], \delta \in[0,1]$, if there exists $P_{1}>0, P_{N_{s}+1}>0, S>0$ such that

$$
\begin{aligned}
& P_{N_{s}+1}>A_{2}^{\prime}\left[(1-\delta) P_{N_{s}+1}+\delta P_{1}\right] A_{2}, \\
& P_{1}>A_{1}^{\prime}\left[(1-\eta)\left(A_{2}^{\prime}\right)^{N_{s}-1} P_{N_{s}+1}\left(A_{2}\right)^{N_{s}-1}+\eta S\right] A_{1}, \\
& S>\left(A_{2}^{\prime}\right)^{i} P_{1} A_{2}^{i} \quad \forall i \in\left\{0,1, \ldots, N_{s}-1\right\},
\end{aligned}
$$

hold, then $K \in \mathbb{K}\left(N_{s}, \eta, \delta\right)$.

Proof. By Theorem 3.1 for $\alpha_{i i}=1$ for all $\mathbb{N}$, and considering the structure of the transition probability matrix in (8.9), we have that system (8.12) is SS if there exists $P \in \mathbb{H}^{2 n+}$, 
$P>0$, such that the following inequalities hold

$$
\begin{aligned}
P_{N_{s}+1} & >A_{2}^{\prime}\left[\delta P_{1}+(1-\delta) P_{N_{s}+1}\right] A_{2}, \\
P_{1} & >A_{1}^{\prime}\left[\left(1-e_{1}\right) P_{1}+e_{1} P_{2}\right] A_{1}, \\
P_{i} & >A_{2}^{\prime}\left[\left(1-e_{i}\right) P_{1}+e_{i} P_{i+1}\right] A_{2},
\end{aligned}
$$

for $i \in\left\{2, \ldots, N_{s}\right\}$. Inequality (8.18) corresponds to (8.15), thus it remains to show that (8.16)-(8.17) implies (8.19)-(8.20). For that, by changing the indexes of (8.17), we have that $S>\left(A_{2}^{\prime}\right)^{i-1} P_{1} A_{2}^{i-1}$ holds for all $i \in\left\{1, \ldots, N_{s}\right\}$, and so by multiplying this inequality by $\left(1-e_{i}\right) \prod_{j=1}^{i-1} e_{j}$ for a fixed $i$, and summing the results up for all $i \in\left\{1, \ldots, N_{s}\right\}$, we get that

$$
\eta S \geq \sum_{i=1}^{N_{s}}\left(1-e_{i}\right)\left(A_{2}^{\prime}\right)^{i-1} P_{1} A_{2}^{i-1} \prod_{j=1}^{i-1} e_{j}
$$

holds, where the term on the left hand side comes from the alternative definition of $\eta$ presented on Lemma 8.1. From (8.16), and considering (8.21) and the definition of $\eta$ taken from (8.8), we can write that

$$
P_{1}>A_{1}^{\prime}\left[\left(1-e_{1}\right) P_{1}+e_{1} \Gamma_{2}\right] A_{1}
$$

holds, where $\Gamma_{2} \triangleq \prod_{i=2}^{N_{s}} e_{i}\left(A_{2}^{\prime}\right)^{N_{s}-1} P_{N_{s}+1}\left(A_{2}\right)^{N_{s}-1}+\sum_{i=2}^{N_{s}}\left(1-e_{i}\right)\left(A_{2}^{\prime}\right)^{i-1} P_{1} A_{2}^{i-1} \prod_{j=2}^{i-1} e_{j}$. We apply a small perturbation $\epsilon_{2}>0$ on (8.22) such that

$$
P_{1}>A_{1}^{\prime}\left[\left(1-e_{1}\right) P_{1}+e_{1}\left(\Gamma_{2}+I \epsilon_{2}\right)\right] A_{1},
$$

also holds. Defining $P_{2}>0$ such that $\Gamma_{2}<P_{2}<\Gamma_{2}+I \epsilon_{2}$ and considering (8.23), we get (8.19). Finally, for showing that (8.20) is satisfied, we define

$$
\begin{aligned}
\Gamma_{m} & \triangleq \prod_{i=m}^{N_{s}} e_{i}\left(A_{2}^{\prime}\right)^{N_{s}-m+1} P_{N_{s}+1}\left(A_{2}\right)^{N_{s}-m+1} \\
& +\sum_{i=m}^{N_{s}}\left(1-e_{i}\right)\left(A_{2}^{\prime}\right)^{i-m+1} P_{1} A_{2}^{i-m+1} \prod_{j=m}^{i-1} e_{j},
\end{aligned}
$$

for $m \in\left\{2, \ldots, N_{s}\right\}$. We can rewrite (8.24) through the following recursive relation

$$
\Gamma_{m}=A_{2}^{\prime}\left[\left(1-e_{m}\right) P_{1}+e_{m} \Gamma_{m+1}\right] A_{2},
$$

for $m \in\left\{2, \ldots, N_{s}-1\right\}$, and $\Gamma_{N_{s}}=A_{2}^{\prime}\left[\left(1-e_{N_{s}}\right) P_{1}+e_{N_{s}} P_{N_{s}+1}\right] A_{2}$. Thus, by beginning 
from $P_{2}>\Gamma_{2}$, and considering the similar reasoning applied to (8.22) and (8.23), we can always apply a small perturbation $\epsilon_{m+1}>0$ on

$$
P_{m}>A_{2}^{\prime}\left[\left(1-e_{m}\right) P_{1}+e_{m} \Gamma_{m+1}\right] A_{2}
$$

such that

$$
P_{m}>A_{2}^{\prime}\left[\left(1-e_{m}\right) P_{1}+e_{m}\left(\Gamma_{m+1}+I \epsilon_{m+1}\right)\right] A_{2}
$$

also holds, for all $m \in\left\{2, \ldots, N_{s}-1\right\}$. By defining $P_{m+1}>0$ such that $\Gamma_{m+1}<P_{m+1}<$ $\Gamma_{m+1}+I \epsilon_{m+1}$, it is always possible to satisfy $P_{m}>A_{2}^{\prime}\left[\left(1-e_{m}\right) P_{1}+e_{m} P_{m+1}\right] A_{2}$ and set $P_{m+1}>\Gamma_{m+1}$ for all $m \in\left\{2, \ldots, N_{s}-1\right\}$, up to $P_{N_{s}}>A_{2}^{\prime}\left[\left(1-e_{N_{s}}\right) P_{1}+e_{N_{s}} P_{N_{s}+1}\right] A_{2}=\Gamma_{N_{s}}$. Thus, we get that (8.20) also holds.

We now provide LMI conditions in Theorem 8.2 below for obtaining $K \in \mathbb{K}\left(N_{s}, \eta, \delta\right)$. In order to ease the exposition, we set

$$
\tilde{\mathcal{A}}_{1} \triangleq\left[\begin{array}{cc}
A_{p} & 0 \\
I_{n} & 0
\end{array}\right], \tilde{\mathcal{A}}_{2} \triangleq\left[\begin{array}{cc}
A_{p} & 0 \\
0 & I_{n}
\end{array}\right], \tilde{\mathcal{B}} \triangleq\left[\begin{array}{c}
B_{p} \\
0
\end{array}\right],
$$

as well as

$$
\tilde{\mathcal{S}}_{i} \triangleq\left[\begin{array}{cc}
\Phi_{i} & 0 \\
0 & I_{n}
\end{array}\right]
$$

where $\Phi_{i} \triangleq \sum_{j=0}^{i-1} A^{j}, i \in\left\{0, \ldots, N_{s}-1\right\}$. We also define the following partitions for the variables $\tilde{G}_{1}, \tilde{G}_{2}, \tilde{Y}$ arising in Theorem 8.2 below

$$
\tilde{G}_{1} \triangleq\left[\begin{array}{cc}
G & G \\
G_{3} & G_{4}
\end{array}\right], \tilde{G}_{2} \triangleq\left[\begin{array}{cc}
G_{1} & G_{2} \\
G & G
\end{array}\right], \tilde{Y}^{\prime} \triangleq\left[\begin{array}{c}
Y^{\prime} \\
Y^{\prime}
\end{array}\right]
$$

along with $\tilde{R}$ and $\tilde{H}_{i}$,

$$
\tilde{R} \triangleq\left[\begin{array}{cc}
R_{1} & R_{2} \\
G & G
\end{array}\right], \tilde{H}_{i} \triangleq\left[\begin{array}{cc}
H_{i 1} & H_{i 2} \\
G & G
\end{array}\right],
$$

for all $i \in\left\{0, \ldots, N_{s}-1\right\}$, where $G \in \mathbb{B}\left(\mathbb{R}^{n}\right)$ and $Y \in \mathbb{B}\left(\mathbb{R}^{n}, \mathbb{R}^{m}\right)$.

Theorem 8.2. For given $N_{s}, \eta \in[0,1]$, and $\delta \in[0,1]$, if there exist $Q_{1}>0, Q_{N+1}>$ $0, M>0, X>0, \tilde{G}_{1}, \tilde{G}_{2}, R \in \mathbb{B}\left(\mathbb{R}^{2 n}\right), \tilde{Y} \in \mathbb{B}\left(\mathbb{R}^{2 n}, \mathbb{R}^{m}\right), \tilde{H}_{i} \in \mathbb{B}\left(\mathbb{R}^{2 n}\right), i \in\left\{0, \ldots, N_{s}-1\right\}$, 
partitioned as in (8.27)-(8.28) such that

$$
\begin{aligned}
& {\left[\begin{array}{ccc}
\operatorname{Her}\left(\tilde{G}_{2}\right)-Q_{N+1} & \bullet & \bullet \\
\delta_{d}\left(\tilde{\mathcal{A}}_{2} \tilde{G}_{2}+\tilde{\mathcal{B}} \tilde{Y}\right) & Q_{N+1} & \bullet \\
\delta_{n}\left(\tilde{\mathcal{A}}_{2} \tilde{G}_{2}+\tilde{\mathcal{B}} \tilde{Y}\right) & 0 & Q_{1}
\end{array}\right]>0} \\
& {\left[\begin{array}{ccc}
\operatorname{Her}\left(\tilde{G}_{1}\right)-Q_{1} & \bullet & \bullet \\
\eta_{d}\left(\tilde{\mathcal{A}}_{1} \tilde{G}_{1}+\tilde{\mathcal{B}} \tilde{Y}\right) & M & \bullet \\
\eta_{n}\left(\tilde{\mathcal{A}}_{1} \tilde{G}_{1}+\tilde{\mathcal{B}} \tilde{Y}\right) & 0 & X
\end{array}\right]>0,} \\
& {\left[\begin{array}{cc}
\operatorname{Her}(\tilde{R})-M & \bullet \\
\left(\tilde{\mathcal{A}}_{2}\right)^{N-1} \tilde{R}+\tilde{\mathcal{S}}_{N-1} \tilde{\mathcal{B}} \tilde{Y} & Q_{N+1}
\end{array}\right]>0,} \\
& {\left[\begin{array}{cc}
\operatorname{Her}\left(\tilde{H}_{i}\right)-X & \bullet \\
\left(\tilde{\mathcal{A}}_{2}\right)^{i} \tilde{H}_{i}+\tilde{\mathcal{S}}_{i} \tilde{\mathcal{B}} \tilde{Y} & Q_{1}
\end{array}\right]>0, \quad \forall i \in\left\{0, \ldots, N_{s}-1\right\},}
\end{aligned}
$$

holds, where $\eta_{n} \triangleq \sqrt{\eta}, \delta_{n} \triangleq \sqrt{\delta}, \eta_{d} \triangleq \sqrt{1-\eta}$, and $\delta_{d} \triangleq \sqrt{1-\delta}$, then by setting $K=Y G^{-1}$, we have that $K \in \mathbb{K}\left(N_{s}, \eta, \delta\right)$.

Proof. The idea is to show that, if (8.29)-(8.32) holds, then by taking $K=Y G^{-1}$, we have that (8.15)-(8.17) is also satisfied. If (8.29)-(8.30) hold, then by writing down the first diagonal blocks of (8.29)-(8.30), we have that

$$
\left[\begin{array}{cc}
\operatorname{Her}\left(G_{1}\right) & \bullet \\
G+G_{2}^{\prime} & \operatorname{Her}(G)
\end{array}\right]>0,\left[\begin{array}{cc}
\operatorname{Her}(G) & \bullet \\
G_{3}+G^{\prime} & \operatorname{Her}\left(G_{4}\right)
\end{array}\right]>0,
$$

and also from (8.31) and (8.32), we have that

$$
\left[\begin{array}{cc}
\operatorname{Her}\left(R_{1}\right) & \bullet \\
G+R_{2}^{\prime} & \operatorname{Her}(G)
\end{array}\right]>0,\left[\begin{array}{cc}
\operatorname{Her}\left(H_{i 1}\right) & \bullet \\
G+H_{i 2}^{\prime} & \operatorname{Her}(G)
\end{array}\right]>0,
$$

for all $i \in\left\{0, \ldots, N_{s}-1\right\}$, thus $G, G_{1}, G_{4}, R_{1}$, and $H_{i 1}$ are nonsingular. By setting $K=Y G^{-1}$ and from (8.30), we can rewrite $\tilde{\mathcal{A}}_{1} \tilde{G}_{1}+\tilde{\mathcal{B}} \tilde{Y}$ as follows

$$
\begin{aligned}
\tilde{\mathcal{A}}_{1} \tilde{G}_{1}+\tilde{\mathcal{B}} \tilde{Y} & =\left[\begin{array}{cc}
A_{p} G+B_{p} Y & A_{p} G+B_{p} Y \\
G & G
\end{array}\right] \\
& =\left[\begin{array}{cc}
A_{p}+B_{p} Y G^{-1} & 0 \\
I & 0
\end{array}\right]\left[\begin{array}{cc}
G & G \\
G_{3} & G_{4}
\end{array}\right] \\
& =A_{1} \tilde{G}_{1} .
\end{aligned}
$$


Furthermore, for (8.29), we have that

$$
\begin{aligned}
\tilde{\mathcal{A}}_{2} \tilde{G}_{2}+\tilde{\mathcal{B} Y} & =\left[\begin{array}{cc}
A_{p} G_{1}+B_{p} Y & A_{p} G_{2}+B_{p} Y \\
G & G
\end{array}\right] \\
& =\left[\begin{array}{cc}
A_{p} & B_{p} Y G^{-1} \\
0 & I
\end{array}\right]\left[\begin{array}{cc}
G_{1} & G_{2} \\
G & G
\end{array}\right] \\
& =A_{2} \tilde{G}_{2},
\end{aligned}
$$

and similarly for (8.32)

$$
\begin{aligned}
\tilde{\mathcal{A}}_{2}{ }^{i} \tilde{H}_{i}+\tilde{\mathcal{S}}_{i} \tilde{\mathcal{B}} \tilde{Y} & =\left[\begin{array}{cc}
A_{p}^{i} & \Phi_{i} B_{p} Y G^{-1} \\
0 & I
\end{array}\right]\left[\begin{array}{cc}
H_{i 1} & H_{i 2} \\
G & G
\end{array}\right] \\
& =\left(A_{2}\right)^{i} \tilde{H}_{i},
\end{aligned}
$$

where $\Phi_{i}=\sum_{j=0}^{i-1} A^{j}$, and $A_{1}$ and $A_{2}$ are the closed-loop matrices given in (8.13). Thus, we can rewrite (8.29)-(8.32) as follows

$$
\begin{aligned}
& {\left[\begin{array}{ccc}
\operatorname{Her}\left(\tilde{G}_{2}\right)-Q_{N+1} & \bullet & \bullet \\
\delta_{d} A_{2} \tilde{G}_{2} & Q_{N+1} & \bullet \\
\delta_{n} A_{2} \tilde{G}_{2} & 0 & Q_{1}
\end{array}\right]>0,} \\
& {\left[\begin{array}{ccc}
\operatorname{Her}\left(\tilde{G}_{1}\right)-Q_{1} & \bullet & \bullet \\
\eta_{d} A_{1} \tilde{G}_{1} & M & \bullet \\
\eta_{n} A_{1} \tilde{G}_{1} & 0 & X
\end{array}\right]>0,} \\
& {\left[\begin{array}{cc}
\operatorname{Her}(\tilde{R})-M & \bullet \\
\left(A_{2}\right)^{N-1} \tilde{R} & Q_{N+1}
\end{array}\right]>0,} \\
& {\left[\begin{array}{cc}
\operatorname{Her}\left(\tilde{H}_{i}\right)-X & \bullet \\
\left(A_{2}\right)^{i} \tilde{H}_{i} & Q_{1}
\end{array}\right]>0, \quad i \in\left\{0, \ldots, N_{s}-1\right\} .}
\end{aligned}
$$

Applying the similar reasoning used in the proofs of Lemmas 4.1 and 4.2, we get that (8.18)-(8.20) hold. Therefore, by Theorem 8.1, $K \in \mathbb{K}\left(N_{s}, \eta, \delta\right)$.

For illustrating the results, we consider the unstable batch reactor as in (WALSH; YE; BUSHNELL, 2002). The system is exactly discretized by means of a zero-order 
hold with rate $T=50 \mathrm{~ms}$, leading to the following values for $A$ and $B$

$$
\begin{aligned}
A & =\left[\begin{array}{rrrr}
1.0795 & -0.0045 & 0.2896 & -0.2367 \\
-0.0272 & 0.8101 & -0.0032 & 0.0323 \\
0.0447 & 0.1886 & 0.7317 & 0.2354 \\
0.0010 & 0.1888 & 0.0545 & 0.9115
\end{array}\right], \\
B^{\prime} & =\left[\begin{array}{rrrr}
0.0006 & 0.2567 & 0.0837 & 0.0837 \\
-0.0239 & 0.0002 & -0.1346 & -0.0046
\end{array}\right] .
\end{aligned}
$$

We study the network-aware design for different characteristics of the $(\eta, \delta)$-SATI by means of Theorem 8.2. For that, we set the maximum packet rate success to $\delta=0.2$ and use the conditions of Theorem 8.1 to construct a controller, if possible, for different values of $N$ and $\eta$. For solving the LMls in Theorem 8.1 and 8.2, we used the parser YALMIP, see (LÖFBERG, 2004), along with the solver MOSEK Aps 8.1, see (MOSEK, 2017). For every $L M I L$ in Theorems 8.1 and 8.2, we impose the restriction $L \geq I \epsilon$, for $\epsilon=1 e^{-4}$. Figure 18 shows whether a stabilizing controller was found by Theorem 8.2, represented by circles, or not, represented by crosses. The solver can find stabilizing

Figure 18 - Solution (circles) or unfeasible (crosses) pairs $(N, \eta)$ of the conditions of Theorem 8.2, for $\delta=0.2$

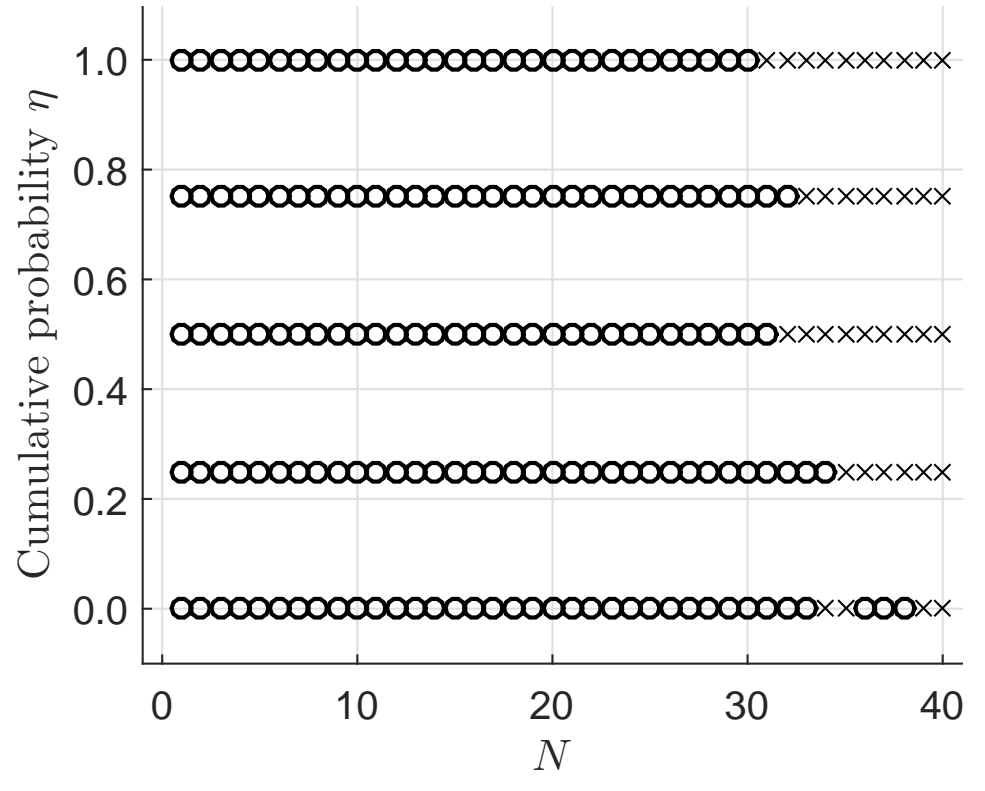

Source: Author, from (OLIVEIRA et al., 2018)

controllers up to $N=30$ for a maximum packet rate success of $\delta=0.2$, even for the worst-case scenario defined by $\eta=0$, that is, when no communication is attempted. Even though the extreme situations of Figure 18 can be achieved, the choice of $\eta$ must 
also be motivated by the system performance over the time, which will be addressed on future work. On the other hand, feasible solutions for (8.29)-(8.32) becomes rarer for $N>30$, and some numerical inconsistencies can be found in terms of $\eta$. For instance, the solver is able to find a controller for $N=31$ and $\eta=0.5$, but it did not find a solution for the pair $N=31$ and $\eta=1.0$, which is not intuitive in the sense that we would expect to obtain solutions for larger values of the cumulative probability $\eta$.

Next, we construct two controllers with the goal of comparing their performance in terms of $N$ and $\eta$. The first one, with gain $K_{\text {SATI }}$, is obtained by means of Theorem 8.2 with $N=2$, a cumulative probability of success of $\eta=0.2$, and a maximum packet rate success of $\delta=0.4$. The second one, with gain $K_{\mathrm{LQR}}$, is designed by solving a discrete-time linear quadratic regulator problem for $Q=I_{4}$ and $R=I_{2}$. The control gains for both cases are given by

$$
\begin{aligned}
& K_{\mathrm{SATI}} \triangleq\left[\begin{array}{rrrr}
0.3330 & -0.6397 & 0.1613 & -0.6449 \\
1.9403 & 0.1250 & 1.6043 & -0.8239
\end{array}\right], \\
& K_{\mathrm{LQR}} \triangleq\left[\begin{array}{rrrr}
0.0153 & -0.8159 & -0.2394 & -0.7515 \\
2.3250 & 0.0801 & 1.6225 & -1.0657
\end{array}\right] .
\end{aligned}
$$

We then study the impact on the stability through Theorem 8.1 for $\delta=0.4$ by increasing $N$ and obtaining the smallest $\eta$ possible such that the closed-loop is still MSS for both controllers. The result is shown in Figure 19. Two interesting behaviors can be noted, the first one is that for both controllers the system is still MSS for $\eta=0$ until some $N$, that is precisely the case where no communication is attempted (lower values of $\eta$ means that fewer power resources are used to communicate). Above this limit, it is necessary to increase the cumulative probability $\eta$ in order to get the MSS of the WNCS, where the maximum value of $N$ in which the WNCS is still MSS is given by $N_{\max }=9$ for the SATI controller and $N_{\max }=6$ for the LQR controller. Thus, the control given by the LQR problem for this choice of $Q$ and $R$ performs worse in terms of the maximum length $N$ than the one calculated via Theorem 8.2. This is intuitive, as the conditions shown in Theorem 8.2 are optimized for the $(\eta, \delta)$-SATI restrictions. Note that $Q$ and $R$ could be chosen in order to improve the behavior in terms of $N$ and $\eta$, even though there are no systematic procedures to tune $Q$ and $R$ for a given SATI. Besides the SATI controller can achieve greater values of $N$ and smaller values of $\eta$ 
Figure 19 - Minimum $\eta$ feasible for the SATI controller (black line) and the LQR controller (dashed grey line).

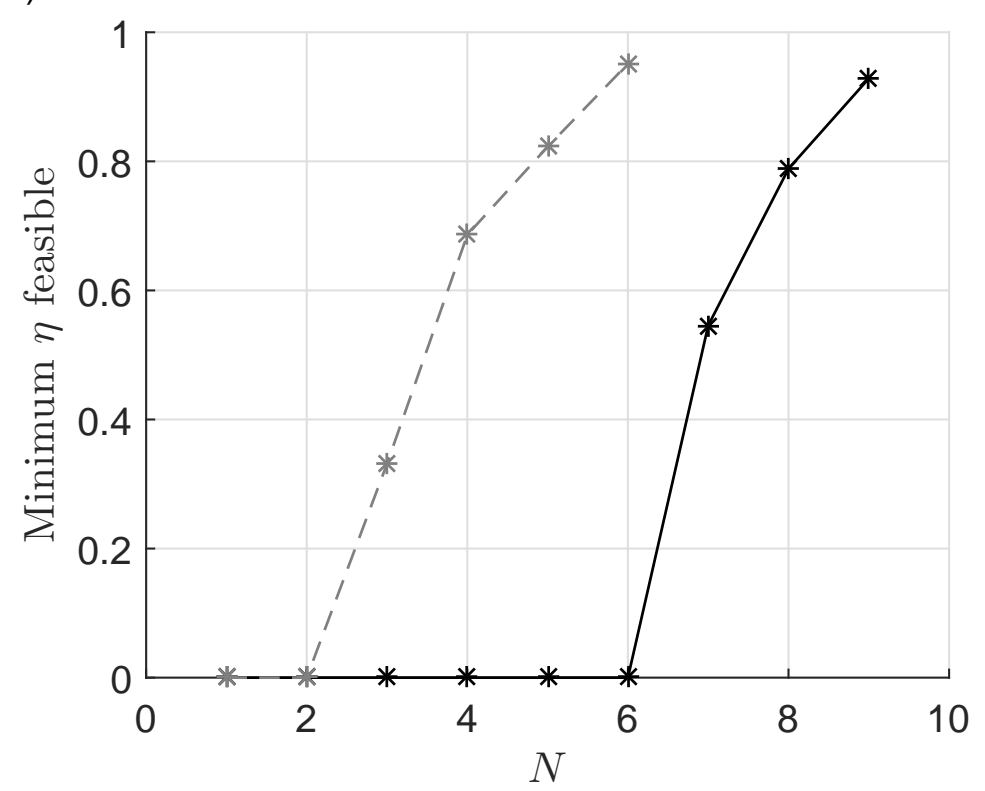

Source: Author, from (OLIVEIRA et al., 2018).

compared to the ones used in the design. 


\section{CONCLUSION AND PERSPECTIVES}

In this thesis, we presented the results obtained during the project "Estimating and control of Markov jump linear systems with partial observation of the operation mode" performed in the Polytechnic School of the University of São Paulo and in the Research Center for Automatic Control of Nancy (CRAN) of the University of Lorraine in France. The motivation lies in extending the knowledge of MJLS considering scenarios of partial observation of the Markov chain, with a special focus on the design of controllers and filters for hidden MJLS. We conclude this work by enumerating our contributions, as well as the perspectives for future research.

- We explored the "limit form" of the $\mathcal{H}_{2}$ norm for hidden MJLS and the relationship between the cluster and mode-independent cases with respect to our setting.

- A new mixed $\mathcal{H}_{2} / \mathcal{H}_{\infty}$ state-feedback control formulation for hidden MJLS was derived in a more general formulation with respect to the one given in (COSTA; MARQUES, 1998). The complete observation case, as well as the cluster and mode-independent approaches, can also be obtained with our method. We also discussed the robust control with respect to uncertain transition and detection probabilities.

- The mixed $\mathcal{H}_{2} / \mathcal{H}_{\infty}$ filtering problem was studied in the hidden MJLS setting, and then the cluster and mode-independent cases were also tackled. We also derived necessary and sufficient conditions for the Bernoulli case.

- An initial study of static output feedback controllers for hidden MJLS is presented, in a mixed $\mathcal{H}_{2} / \mathcal{H}_{\infty}$ control setting. The philosophy is based on the classical two steps procedure that can be found in the literature.

- A new form of obtaining $\mathcal{H}_{2}, \mathcal{H}_{\infty}$, and mixed $\mathcal{H}_{2} / \mathcal{H}_{\infty}$ dynamic output feedback controllers is presented. The conditions are given in the BMl formulation and suggest a type of ad-hoc separation procedure in the vein of (OLIVEIRA; GEROMEL; BERNUSSOU, 2000), in which a stabilizing state-feedback controller is given as an input to obtain the remaining controller matrices. Due to some properties of 
the hidden MJLS, the cluster and mode-independent cases can also be obtained, providing a new tool for obtaining this type of controllers in the literature. The robust case is also investigated.

- Some interesting applications were considered in the context of AFTCS and NCS. In the latter, the concept of $(\eta, \delta)$-SATI for WNCS was deepened, and a new stability condition, as well as co-design methods for state-feedback controllers, were derived.

Finally, a new Riccati-like $\mathcal{H}_{\infty}$ condition that generalizes the results in (COSTA; MARQUES, 1998) was obtained in (OLIVEIRA; COSTA, 2017c), that may be an alternative to mixed $\mathcal{H}_{2} / \mathcal{H}_{\infty}$ applications with respect to the bounded-real lemma derived (TODOROV; FRAGOSO; COSTA, 2018).

There is a great deal of possibilities for future research. Some of them are mentioned below:

- The implementation of the "detector approach" in real applications is still an open point. The question is, given an FDI algorithm (or an acknowledgment scheme) and its statistics, how well could we approximate its behavior with our formulation?

- Is it possible to derive necessary and sufficient conditions for the stabilizability of discrete-time hidden MJLS as posed for the continuous-time formulation presented (FRAGOSO; COSTA, 2004) and (COSTA; FRAGOSO; TODOROV, 2013)? If so, is it possible to extend such result for filters and more complex controller structures?

- The assumption that the filtered system must be stochastically stable seems very restricting. Is it possible to overcome it in the contexts of partial observation?

- Even though we provided an interesting form of obtaining dynamic output feedback controllers, the parametrization of all controllers for this type of problem remains an open (and very challenging) problem in the literature.

- Is there "dual-like" $\mathcal{H}_{\infty}$ conditions with respect to the bounded-real lemma of (TODOROV; FRAGOSO; COSTA, 2018) (or even for the mode-dependent case of 
(SEILER; SENGUPTA, 2003))? The answers to this question could unveil MJLS design conditions for observers and dynamic controllers echoing a type of $\mathcal{H}_{\infty}$ "separation property" as presented in (DOYLE et al., 1989) for the continuoustime LTI systems, and in (STOORVOGEL, 1992), for the discrete-time case.

- Regarding the static output feedback control, exploring new tools of obtaining such controllers is desirable. The dependence on $K$ in the procedure may lead to undesirable behaviors.

- There are still open points that are worth studying in the AFTCS approach of (MAHMOUD; JIANG; ZHANG, 2003; ABERKANE, 2009) for the discretetime setting and the relationship between the "detector-approach" of (COSTA; FRAGOSO; TODOROV, 2015) and the AFTCS approach of (MAHMOUD; JIANG; ZHANG, 2003; ABERKANE, 2009) in a design context.

- The use of $y$ for estimating $\theta$, and consequently applying that estimation in control problems, is a challenging and very complex problem.

- Concerning the $(\eta, \delta)-$ SATI, the extension for optimal and $\mathcal{H}_{\infty}$ control is a topic of interest. Besides, the co-design problem for different controller structures is needed, since it is not always possible to perfectly measure the system's states. Finally, the sufficient-only stability conditions that are based on MJLS theory may present some undesirable properties. More research is needed in this aspect of the theory. 


\section{REFERENCES}

ABERKANE, S. Output feedback $H_{2} / H_{\infty}$ control of a class of discrete-time reconfigurable control systems. IFAC Proceedings Volumes, v. 42, n. 8, p. 698-703, 2009. 7th IFAC Symposium on Fault Detection, Supervision and Safety of Technical Processes.

. Reconfigurable control systems: A nonhomogeneous Markovian jump system approach. IFAC Proceedings Volumes, v. 44, n. 1, p. 5425-5429, 2011. 18th IFAC World Congress.

ABERKANE, S. et al. Output feedback control of a class of stochastic hybrid systems. Automatica, v. 44, n. 5, p. 1325-1332, 2008.

ABERKANE, S.; PONSART, J. C.; SAUTER, D. Multiobjective output feedback control of a class of stochastic hybrid systems with state-dependent noise. Mathematical Problems in Engineering, v. 2007, n. 31561, p. 26, 2007.

. Output-feedback $H_{2} / H_{\infty}$ control of a class of networked fault tolerant control systems. Asian Journal of Control, v. 10, n. 1, p. 24-33, 2008.

ABERKANE, S.; SAUTER, D.; PONSART, J. C. Output feedback robust $H_{\infty}$ control of uncertain active fault tolerant control systems via convex analysis. International Journal of Control, v. 81, n. 2, p. 252-263, 2008.

BAR-SHALOM, Y.; LI, X.-R.; KIRUBARAJAN, T. Estimation with Applications to Tracking and Navigation. New York City: John Wiley \& Sons, Inc., 2002. ISBN 9780471416555.

BERNSTEIN, D. S.; HADDAD, W. M. LQG control with an $H_{\infty}$ performance bound: a Riccati equation approach. IEEE Transactions on Automatic Control, v. 34, n. 3, p. 293-305, 1989.

BLAIR, W. P.; SWORDER, D. D. Feedback control of a class of linear discrete systems with jump parameters and quadratic cost criteria. International Journal of Control, v. 21 , n. 5, p. 833-844, 1975.

BOUKAS, E. K. Static output feedback control for stochastic hybrid systems: LMI approach. Automatica, v. 42, n. 1, p. 183-188, 2006.

Stochastic Switching Systems: Analysis and Design. New York City: Birkhäuser Basel, 2006. ISBN 978-0-8176-3782-8.

BOUKAS, E. K.; SHI, P. $H_{\infty}$ control for discrete-time linear systems with Markovian jumping parameters. In: IEEE CONFERENCE ON DECISION AND CONTROL, 36th., 1997, San Diego, CA. Proceedings... New York City: IEEE, 1997. p. 4134-4139. 
BOYD, S. et al. Linear Matrix Inequalities in System and Control Theory. Philadelphia, PA: Society for Industrial and Applied Mathematics, 1994. (SIAM Studies in Applied and Numerical Mathematics). ISBN 0-89871-334-X.

CARNEVALE, D.; TEEL, A. R.; NESIC, D. A Lyapunov proof of an improved maximum allowable transfer interval for networked control systems. IEEE Transactions on Automatic Control, v. 52, n. 5, p. 892-897, 2007.

CHE, W. W.; WANG, J. L. Static output feedback $H_{\infty}$ control for discrete-time Markov jump linear systems. In: IEEE ICCA, 8th., 2010, Xiamen, China. Proceedings... New York City: IEEE, 2010. p. 2278-2283.

CHEN, M. et al. Finite-time asynchronous $H_{\infty}$ control for Markov jump repeated scalar non-linear systems with input constraints. Applied Mathematics and Computation, v. 275 , p. 172-180, 2016.

CHITRAGANTI, S. Sur la stabilité et la commande des système linéaires à sauts aléatoire. 150 p. Tese (Doutorado) — Université de Lorraine, Nancy, 2014.

CHIZECK, H. J.; JI, Y. Optimal quadratic control of jump linear systems with Gaussian noise in discrete-time. In: IEEE CONFERENCE ON DECISION AND CONTROL, 27th., 1988, Austin, TX. Proceedings... New York City: IEEE, 1988. p. 1989-1993.

CHIZECK, H. J.; WILLSKY, A. S.; CASTANON, D. Discrete-time Markovian-jump linear quadratic optimal control. International Journal of Control, v. 43, n. 1, p. 213-231, 1986.

COSTA, E. F.; SAPORTA, B. de. Linear minimum mean square filters for Markov jump linear systems. IEEE Transactions on Automatic Control, v. 62, n. 7, p. 3567-3572, 2017.

COSTA, E. F.; VAL, J. B. R. do. On the detectability and observability of discrete-time Markov jump linear systems. Systems \& Control Letters, v. 44, n. 2, p. 135-145, 2001.

On the observability and detectability of continuous-time Markov jump linear systems. SIAM Journal on Control and Optimization, v. 41, n. 4, p. 1295-1314, 2002.

. Weak detectability and the linear-quadratic control problem of discrete-time Markov jump linear systems. International Journal of Control, Taylor \& Francis, v. 75, n. 16-17, p. 1282-1292, 2002.

COSTA, O. L. V. Linear minimum mean square error estimation for discrete-time Markovian jump linear systems. IEEE Transactions on Automatic Control, v. 39, p. 1685-1689, 1994.

Discrete-time coupled Riccati equations for systems with Markov switching parameters. Journal of Mathematical Analysis and Applications, v. 194, n. 1, p. 197-216, 1995.

COSTA, O. L. V.; FRAGOSO, M. D. Discrete-time LQ-optimal control problems for infinite Markov jump parameter systems. IEEE Transactions on Automatic Control, v. 40, n. 12 , p. 2076-2088, 1995. 
COSTA, O. L. V.; FRAGOSO, M. D.; MARQUES, R. P. Discrete-Time Markov Jump Linear Systems. New York City: Springer-Verlag London, 2005. ISBN 1852337613.

COSTA, O. L. V.; FRAGOSO, M. D.; TODOROV, M. G. Continuous-Time Markov Jump Linear Systems. New York City: Springer-Verlag Berlin Heidelberg, 2013. ISBN 978-3-642-34099-4.

. A detector-based approach for the $H_{2}$ control of Markov jump linear systems with partial information. IEEE Transactions on Automatic Control, v. 60, n. 5, p. 1219-1234, 2015.

COSTA, O. L. V.; GUERRA, S. Robust linear filtering for discrete-time hybrid Markov linear systems. International Journal of Control, Taylor \& Francis, v. 75, n. 10, p. 712-727, 2002.

Stationary filter for linear minimum mean square error estimator of discrete-time Markovian jump systems. IEEE Transactions on Automatic Control, v. 47, n. 8, p. 1351-1356, 2002.

COSTA, O. L. V.; MARQUES, R. P. Mixed $H_{2} / H_{\infty}$-control of discrete-time Markovian jump linear systems. IEEE Transactions on Automatic Control, v. 43, n. 1, p. 95-100, 1998.

COSTA, O. L. V.; TUESTA, E. F. Finite horizon quadratic optimal control and a separation principle for Markovian jump linear systems. IEEE Transactions on Automatic Control, v. 48, n. 10, p. 1836-1842, 2003.

$\mathrm{H}_{2}$-control and the separation principle for discrete-time Markovian jump linear systems. Mathematics of Control, Signals and Systems, v. 16, n. 4, p. 320-350, 2004.

COSTA, O. L. V.; VAL, J. B. R. do. Full information $H_{\infty}$-control for discrete-time infinite Markov jump parameter systems. Journal of Mathematical Analysis and Applications, v. 202, n. 2, p. 578-603, 1996.

COSTA, O. L. V.; VAL, J. B. R. do; GEROMEL, J. C. A convex programming approach to $\mathrm{H}_{2}$ control of discrete-time Markovian jump linear systems. International Journal of Control, v. 66, n. 4, p. 557-580, 1997.

DAAFOUZ, J.; BERNUSSOU, J. Parameter dependent Lyapunov functions for discrete time systems with time varying parametric uncertainties. Systems \& Control Letters, v. 43, n. 5 , p. 355-359, 2001.

DAVIS, M. H. A.; VINTER, R. B. Stochastic Modelling and Control. New York City: Springer Netherlands, 1985. ISBN 978-94-010-8640-0.

DONKERS, M. C. F. et al. Stability analysis of networked control systems using a switched linear systems approach. IEEE Transactions on Automatic Control, v. 56, n. 9, p. 2101-2115, 2011.

DOYLE, J. C. et al. State-space solutions to standard $H_{2}$ and $H_{\infty}$ control problems. IEEE Transactions on Automatic Control, v. 34, n. 8, p. 831-847, 1989. 
DRAGAN, V.; MOROZAN, T.; STOICA, A.-M. Mathematical Methods in Robust Control of Linear Stochastic Systems. New York City: Springer, 2013. ISBN 978-1-4419-0629-8.

DUCARD, G. J. J. Fault-tolerant Flight Control and Guidance Systems. New York City: Springer-Verlag London, 2009. ISBN 978-1-84882-560-4.

FIORAVANTI, A. R.; GONÇALVES, A. P. C.; GEROMEL, J. C. $H_{2}$ filtering of discretetime Markov jump linear systems through linear matrix inequalities. International Journal of Control, v. 81, n. 8, p. 1221-1231, 2008.

. Discrete-time $H_{\infty}$ output feedback for Markov jump systems with uncertain transition probabilities. International Journal of Robust and Nonlinear Control, v. 23, n. 8, p. 894-902, 2013.

. Optimal $H_{2}$ and $H_{\infty}$ mode-independent control for generalized Bernoulli jump systems. ASME. Journal Dynamic Systems, Measurement and Control, v. 136, n. 4, p. 011004-011004-6, 2013.

. Optimal $\mathrm{H}_{2}$ and $\mathrm{H}_{\infty}$ mode-independent filters for generalised Bernoulli jump systems. International Journal of Systems Sciences, v. 46, n. 3, p. 405-417, 2015.

FIORAVANTI, A. R. et al. Obtaining alternative LMI constraints with applications to discrete-time MJLS and switched systems. Journal of the Franklin Institute, v. 350, n. 8, p. 2212-2228, 2013.

FLORENTIN, J. J. Optimal control of continuous time, Markov, stochastic systems. Journal of Electronics and Control, v. 10, n. 6, p. 473-488, 1961.

FRAGOSO, M. D. Discrete-time jump LQG problem. International Journal of Systems Science, v. 20, n. 12, p. 2539-2545, 1989.

FRAGOSO, M. D.; COSTA, O. L. V. Mean square stabilizability of continuous-time linear systems with partial information on the Markovian jumping parameters.

Stochastic Analysis and Applications, Taylor \& Francis, v. 22, n. 1, p. 99-111, 2004.

FRAGOSO, M. D.; VAL, J. B. R. do; JR., D. L. P. $H_{\infty}$-control for linear systems with Markovian jumping parameters. Control Theory and Advanced Technology, v. 10, n. 4, p. 1459-1474, 1995.

GAHINET, P. et al. LMI Control Toolbox: For Use with MATLAB. User's Guide. Version 1. Natick, MA, 1995. (Computation, visualization, programming).

GEROMEL, J. C.; BERNUSSOU, J.; OLIVEIRA, M. C. de. $H_{2}$-norm optimization with constrained dynamic output feedback controllers: decentralized and reliable control. IEEE Transactions on Automatic Control, v. 44, n. 7, p. 1449-1454, 1999.

GEROMEL, J. C.; GONÇALVES, A. P. C.; FIORAVANTI, A. R. Dynamic output feedback control of discrete-time Markov jump linear systems through linear matrix inequalities. SIAM Journal on Control and Optimization, v. 48, n. 2, p. 573-593, 2009. 
GEROMEL, J. C.; PERES, P. L. D.; SOUZA, S. R. Convex analysis of output feedback structural constraints. In: IEEE CONFERENCE ON DECISION AND CONTROL, 32nd., 1993, San Antonio, TX. Proceedings... New York City: IEEE, 1993. p. 1363-1364.

A convex approach to the mixed $\mathcal{H}_{2} / \mathcal{H}_{\infty}$ control problem for discrete-time uncertain systems. SIAM Journal on Control Optimization, Society for Industrial and Applied Mathematics, Philadelphia, PA, v. 33, n. 6, p. 1816-1833, 1995.

GHAOUI, L. E.; RAMI, M. A. Robust state-feedback stabilization of jump linear systems via LMls. International Journal of Robust and Nonlinear Control, v. 6 , n. 9-10, p. 1015-1022, 1996.

GONÇALVES, A. P.; FIORAVANTI, A. R.; GEROMEL, J. C. Markov jump linear systems and filtering through network transmitted measurements. Signal Processing, v. 90 , n. 10 , p. $2842-2850,2010$.

GONÇALVES, A. P. C. State-feeedback control for Markovian systems (portuguese). Campinas, SP, Brazil: University of Campinas (UNICAMP), 2014. 12-14 p. Available in <http://www.dsce.fee.unicamp.br/ alimped/ia361_slides_4_ 2014.pdf>. Visited on 21 Jan. 2019.

GONÇALVES, A. P. C. et al. $H_{\infty}$ state feedback control of discrete-time Markov jump linear systems through linear matrix inequalities. IFAC Proceedings Volumes, v. 44, n. 1, p. 12620-12625, 2011.

GONÇALVES, A. P. C.; FIORAVANTI, A. R.; GEROMEL, J. C. $H_{\infty}$ filtering of discrete-time Markov jump linear systems through linear matrix inequalities. IEEE Transactions on Automatic Control, v. 54, n. 6, p. 1347-1351, 2009.

. $H_{\infty}$ robust and networked control of discrete-time MJLS through LMls. Journal of the Franklin Institute, v. 349, n. 6, p. 2171-2181, 2012.

GONÇALVES, A. P. C.; GEROMEL, J. C.; FIORAVANTI, A. R. Filtering of discrete-time Markov jump linear systems with uncertain transition probabilities. International Journal of Robust and Nonlinear Control, v. 21, n. 6, p. 613-624, 2011.

HEEMELS, W. P. M. H.; WOUW, N. van de. Stability and stabilization of networked control systems. In: BEMPORAD, A.; HEEMELS, M.; JOHANSSON, M. (Ed.).

Networked Control Systems. London: Springer, 2010. v. 406 of the series Lecture Notes in Control and Information Sciences, p. 203-253. ISBN 978-0-85729-033-5.

HESPANHA, J. P.; NAGHSHTABRIZI, P.; XU, Y. A survey of recent results in networked control systems. Proceedings of the IEEE, v. 95, n. 1, p. 138-162, 2007.

HWANG, I. et al. A survey of fault detection, isolation, and reconfiguration methods. IEEE Transactions on Control Systems Technology, v. 18, n. 3, p. 636-653, 2010.

JI, Y.; CHIZECK, H. J. Controllability, observability and discrete-time Markovian jump linear quadratic control. International Journal of Control, v. 48, n. 2, p. 481-498, 1988. 
JIANG, J.; YU, X. Fault-tolerant control systems: A comparative study between active and passive approaches. Annual Reviews in Control, v. 36, n. 1, p. 60-72, 2012.

KHARGONEKAR, P. P.; ROTEA, M. A. Mixed $H_{2} / H_{\infty}$ control: a convex optimization approach. IEEE Transactions on Automatic Control, v. 36, n. 7, p. 824-837, 1991.

KHARGONEKAR, P. P.; ROTEA, M. A.; BAEYENS, E. Mixed $H_{2} / H_{\infty}$ filtering. International Journal of Robust and Nonlinear Control, v. 6, n. 4, p. 313-330, 1996.

LEON-GARCIA, A. Probability, Statistics, and Random Processes for Electrical Engineering. 3rd. ed. Upper Saddle River, NJ: Pearson/Prentice Hall, 2008. ISBN 978-0-13-147122-1.

LIU, X. et al. Dynamic output feedback asynchronous control of networked Markovian jump systems. IEEE Transactions on Systems, Man, and Cybernetics: Systems, p. 1-11, 2018.

LÖFBERG, J. YALMIP : A toolbox for modeling and optimization in MATLAB. In: IEEE CACSD CONFERENCE, 2004, Taipei, Taiwan. Proceedings... New York City: IEEE, 2004.

MAHMOUD, M.; JIANG, J.; ZHANG, Y. Active Fault Tolerant Control Systems - Stochastic Analysis and Synthesis. Germany: Springer Verlag, 2003. ISBN 3-540-00318-5.

MARITON, M. Detection delays, false alarm rates and the reconfiguration of control systems. International Jounal of Control, v. 49, p. 981-992, 1989.

. Jump Linear Systems in Automatic Control. New York City: CRC Press, 1990. ISBN 978-0824782009.

MARITON, M.; BERTRAND, P. Output feedback for a class of linear systems with stochastic jump parameters. IEEE Transactions on Automatic Control, v. 30, n. 9, p. 898-900, 1985.

MEHDI, D.; BOUKAS, E. K.; BACHELIER, O. Static output feedback design for uncertain linear discrete time systems. IMA Journal of Mathematical Control and Information, v. 21, n. 1, p. 1-13, 2004.

MORAIS, C. F. et al. $\mathcal{H}_{\infty}$ static output feedback control of discrete-time Markov jump linear systems with uncertain transition probability matrix. In: AMERICAN CONTROL CONFERENCE, 2014, Portland, OR. Proceedings... New York City: IEEE, 2014. p. 489-494.

. An LMI approach for $H_{2}$ dynamic output feedback control of discrete-time Markov systems with uncertain probabilities. In: IEEE CONFERENCE ON COMPUTER AIDED CONTROL SYSTEM DESIGN (CACSD), 2016, Buenos Aires. Proceedings... New York City: IEEE, 2016. p. 1-6.

LMI-based design of $H_{\infty}$ dynamic output feedback controllers for mjls with uncertain transition probabilities. In: AMERICAN CONTROL CONFERENCE (ACC), 2016, Boston, MA. Proceedings... New York City: IEEE, 2016. p. 5650-5655. 
. Reduced-order dynamic output feedback control of uncertain discrete-time Markov jump linear systems. International Journal of Control, v. 90, n. 11, p. 2368-2383, 2017.

An LMI approach for $H_{2}$ and $H_{\infty}$ reduced-order filtering of uncertain discretetime Markov and Bernoulli jump linear systems. Automatica, v. 95, p. 463-471, 2018.

MOROZAN, T. Stabilization of some stochastic discrete-time control systems. Stochastic Analysis and Applications, Taylor \& Francis, v. 1, n. 1, p. 89-116, 1983.

MOSEK, A. MOSEK Optimization Toolbox for MATLAB 8.1.0.38. Copenhagen, Denmark, 2017. Available in <https://docs.mosek.com/8.1/toolbox/index.html>. Visited on 21 Jan. 2019.

NESIC, D.; TEEL, A. R. Input-output stability properties of networked control systems. IEEE Transactions on Automatic Control, v. 49, p. 1650-1667, 2004.

OGURA, M. et al. State-feedback control of Markov jump linear systems with hidden-Markov mode observation. Automatica, v. 89, p. 65-72, 2018.

OLIVEIRA, A. M. de. Análise e Controle de um Sistema Mecânico com Dados Transmitidos Através da Rede. 129 p. Dissertação (Mestrado) - University of Campinas (UNICAMP), Campinas, 2015.

OLIVEIRA, A. M. de; COSTA, O. L. V. Controle misto $H_{2} / H_{\infty}$ para sistemas lineares com saltos Markovianos com cadeia oculta. In: CONGRESSO BRASILEIRO DE AUTOMÁTICA, XXI., 2016, Vitória, ES. Anais Eletrônicos... Campinas: SBA, 2016. p. $146-151$.

$H_{\infty}$-filtering design for discrete-time Markov jump systems with hidden parameters. In: IEEE CONFERENCE ON CONTROL APPLICATIONS (CCA), 2016, Buenos Aires. Proceedings... New York City: IEEE, 2016. p. 972-977.

$\mathrm{H}_{2}$-filtering for discrete-time hidden Markov jump systems. International Journal of Control, v. 90, n. 3, p. 599-615, 2017.

. $\mathcal{H}_{\infty}$-filtering for Markov jump linear systems with partial information on the jump parameter. IFAC Journal of Systems and Control, v. 1, p. 13-23, 2017.

. Mixed $H_{2} / H_{\infty}$ state feedback control for Markov jump linear systems with hidden observations. IFAC-PapersOnLine, v. 50, n. 1, p. 3800-3805, 2017. 20th IFAC World Congress.

Mixed $\mathcal{H}_{2} / \mathcal{H}_{\infty}$ control of hidden Markov jump systems. International Journal of Robust and Nonlinear Control, v. 28, n. 4, p. 1261-1280, 2018.

Mixed $\mathcal{H}_{2} / \mathcal{H}_{\infty}$ filtering for Markov jump linear systems. International Journal of Systems Science, v. 49, n. 15, p. 3023-3036, 2018.

OLIVEIRA, A. M. de; COSTA, O. L. V.; DAAFOUZ, J. Controle $\mathcal{H}_{\infty}$ por realimentação estática de saída para sistemas lineares com saltos markovianos com observação parcial. In: CONGRESSO BRASILEIRO DE AUTOMÁTICA (CBA 2018), XXII., 2018, João Pessoa, PB. Anais Eletrônicos... Campinas: SBA, 2018. 
. Design of stabilizing dynamic output feedback controllers for hidden Markov jump linear systems. IEEE Control Systems Letters, v. 2, n. 2, p. 278-283, 2018.

A suboptimal LMI formulation for the $\mathcal{H}_{2}$ static output feedback control of hidden Markov jump linear systems. In: EUROPEAN CONTROL CONFERENCE (ECC), 2018, Limassol, Cyprus. Proceedings... New York City: IEEE, 2018. p. 3095-3100.

$\mathcal{H}_{2}$ dynamic output feedback control for hidden Markov jump linear systems. In:

IMA Volume for Stochastic Control, Computational Methods, and Applications. New York City: Springer International Publishing, 2019. To appear.

OLIVEIRA, A. M. de et al. Network-aware design of state-feedback controllers for linear wireless networked control systems. IFAC-PapersOnLine, v. 51, n. 16, p. 205-210, 2018. 6th IFAC Conference on Analysis and Design of Hybrid Systems ADHS 2018.

OLIVEIRA, M. C. de. Controle de sistemas lineares baseado nas desigualdades matriciais lineares (portuguese). 170 p. Tese (Doutorado) - University of Campinas, Campinas, 1999.

OLIVEIRA, M. C. de; BERNUSSOU, J.; GEROMEL, J. C. A new discrete-time robust stability condition. Systems \& Control Letters, v. 37, n. 4, p. 261-265, 1999.

OLIVEIRA, M. C. de; GEROMEL, J. C.; BERNUSSOU, J. Design of dynamic output feedback decentralized controllers via a separation procedure. International Journal of Control, Taylor \& Francis, v. 73, n. 5, p. 371-381, 2000.

. Extended $H_{2} / H_{\infty}$ characterizations and controller parametrizations for discrete-time systems. International Journal of Control, Taylor \& Francis, v. 75, n. 9, p. 666-679, 2002.

PATTON, R. J. Fault-tolerant control systems: The 1997 situation. In: IFAC SYMPOSIUM ON FAULT DETECTION SUPERVISION AND SAFETY FOR TECHNICAL PROCESSES, 1997, Hull, UK. Proceedings... Kidlington Oxford, UK: IFAC, 1997. p. 1033-1054.

PEAUCELLE, D.; ARZELIER, D. An efficient numerical solution for $\mathrm{H}_{2}$ static output feedback synthesis. In: EUROPEAN CONTROL CONFERENCE (ECC), 2001, Porto, Portugal. Proceedings... New York City: IEEE, 2001. p. 3800-3805.

POSTOYAN, R.; NEŠIĆ, D. Time-triggered control of nonlinear discrete-time systems. In: IEEE CONFERENCE ON DECISION AND CONTROL, 55th., 2016, Las Vegas, NV. Proceedings... New York City: IEEE, 2016. p. 6814-6819.

RAMI, M. A.; GHAOUI, L. E. Robust stabilization of jump linear systems using linear matrix inequalities. In: IFAC SYMPOSIUM ON ROBUST CONTROL DESIGN, 1994, Rio de Janeiro. Proceedings... Kidlington Oxford, UK: IFAC, 1994. p. 148-151.

RODRIGUES, C. C. G.; TODOROV, M. G.; FRAGOSO, M. D. A bounded real lemma for continuous-time linear systems with partial information on the Markovian jumping parameters. In: IEEE CONFERENCE ON DECISION AND CONTROL (CDC), 54th., 2015, Osaka, Japan. Proceedings... New York City: IEEE, 2015. p. 4226-4231. 
ROSS, S. M. Introduction to Probability Models. 10th. ed. San Diego, CA: Academic Press, Inc., 2010. ISBN 978-0-12-375686-2.

SADABADI, M. S.; PEAUCELLE, D. From static output feedback to structured robust static output feedback: A survey. Annual Reviews in Control, v. 42, p. 11-26, 2016.

SÄRKKÄ, S. Bayesian Filtering and Smoothing. New York City: Cambridge University Press, 2013. ISBN 1107619289,9781107619289.

SCHERER, C.; GAHINET, P.; CHILALI, M. Multiobjective output-feedback control via LMI optimization. IEEE Transactions on Automatic Control, v. 42, n. 7, p. 896-911, 1997.

SEILER, P.; SENGUPTA, R. A bounded real lemma for jump systems. IEEE Transactions on Automatic Control, v. 48, n. 9, p. 1651-1654, 2003.

An $H_{\infty}$ approach to networked control. IEEE Transactions on Automatic Control, v. 50, n. 3, p. 356-364, 2005.

SILVA, C. A. Algoritmos para o custo médio a longo prazo de sistemas com saltos markovianos parcialmente observados. $120 \mathrm{p}$. Tese (Doutorado) Universidade de São Paulo, São Carlos, 2012.

SILVA, C. A.; BORTOLIN, D. C.; COSTA, E. F. An algorithm for the long run average cost problem for linear systems with indirect observation of Markov jump parameters. IFAC Proceedings Volumes, v. 44, n. 1, p. 12668 - 12673, 2011. 18th IFAC World Congress.

SONG, J. et al. Finite-time $l_{2}-l_{\infty}$ control of Markovian jump linear systems with partly accessible hidden information via asynchronous output feedback. In: ASIAN CONTROL CONFERENCE (ASCC), 11th., 2017, Gold Coast, Queensland, Australia. Proceedings... New York City: IEEE, 2017. p. 2447-2452.

SOUZA, C. E. de. A mode-independent $H_{\infty}$ filter design for discrete-time Markovian jump linear systems. In: IEEE INTERNATIONAL CONFERENCE ON DECISION AND CONTROL, 42nd., 2003, Maui, HI. Proceedings... New York City: IEEE, 2003. p. 2811-2816.

. Robust stability and stabilization of uncertain discrete-time Markovian jump linear systems. IEEE Transactions on Automatic Control, v. 51, n. 5, p. 836-841, 2006.

SOUZA, C. E. de; FRAGOSO, M. D. $H_{\infty}$-control for linear systems with Markovian jumping parameters. Control Theory and Advanced Technology, v. 9, p. 457-466, 1993.

. $H_{\infty}$ filtering for discrete-time linear systems with Markovian jumping parameters. In: IEEE CONFERENCE ON DECISION AND CONTROL, 36th., 1997, San Diego, CA. Proceedings... New York City: IEEE, 1997. p. 2181-2186.

. $H_{\infty}$ filtering for discrete-time linear systems with Markovian jumping parameters. International Journal of Robust and Nonlinear Control, John Wiley \& Sons, Ltd., v. 13, n. 14, p. 1299-1316, 2003. 
SRICHANDER, R.; WALKER, B. K. Stochastic stability analysis for continuous-time fault tolerant control systems. International Journal of Control, v. 57, n. 2, p. 433-452, 1993.

STADTMANN, F.; COSTA, O. L. V. $H_{2}$-control of continuous-time hidden Markov jump linear systems. IEEE Transactions on Automatic Control, v. 62, n. 8, p. 4031-4037, 2017.

Exponential hidden Markov models for $H_{\infty}$ control of jumping systems. IEEE Control Systems Letters, v. 2, n. 4, p. 845-850, 2018.

STEIN, G. Respect the unstable. IEEE Control Systems, v. 23, n. 4, p. 12-25, 2003.

StOORVOGEL, A. A. The $\boldsymbol{H}_{\infty}$ Control Problem: A State Space Approach. Upper Saddle River, NJ: Prentice-Hall, 1992. ISBN 978-0133880670.

SWORDER, D. Feedback control of a class of linear systems with jump parameters. IEEE Transactions on Automatic Control, v. 14, n. 1, p. 9-14, 1969.

SWORDER, D. D.; CHOU, D. S. Feedforward/feedback controls in a noisy environment. IEEE Transactions on Systems, Man, and Cybernetics, v. 16, n. 4, p. 522-531, 1986.

TODOROV, M. G.; FRAGOSO, M. A new look at the robust control of discrete-time Markov jump linear systems. International Journal of Control, v. 89, n. 3, p. 518-534, 2016.

TODOROV, M. G.; FRAGOSO, M. D. New results on the robustness of discrete-time Markov jump linear systems. In: IEEE CONFERENCE ON DECISION AND CONTROL (CDC), 51st., 2012, Maui, HI. Proceedings... New York City: IEEE, 2012. p. $1331-1336$.

TODOROV, M. G.; FRAGOSO, M. D.; COSTA, O. L. V. A new approach for the $H_{\infty}$ control of Markov jump linear systems with partial information. In: IEEE CONFERENCE ON DECISION AND CONTROL (CDC), 54th., 2015, Osaka, Japan. Proceedings... New York City: IEEE, 2015. p. 3592-3597.

. Detector-based $H_{\infty}$ results for discrete-time Markov jump linear systems with partial observations. Automatica, v. 91, p. 159-172, 2018.

TRANTER, W. et al. Principles of Communication Systems Simulation with Wireless Applications. First. Upper Saddle River, NJ: Prentice Hall Press, 2003. ISBN 0134947908.

VAL, J. B. do; GEROMEL, J. C.; GONÇALVES, A. P. C. The $H_{2}$-control for jump linear systems: cluster observations of the Markov state. Automatica, Elsevier, v. 38, n. 2, 2002.

VARGAS, A. N.; COSTA, E. F.; VAL, B. R. do. On the control of Markov jump linear systems with no mode observation: application to a DC motor device. International Journal of Robust and Nonlinear Control, v. 23, n. 10, p. 1136-1150, 2012. 
VARGAS, A. N.; COSTA, E. F.; VAL, J. do. Advances in the Control of Markov Jump Linear Systems with No Mode Observation. New York City: Springer International Publishing, 2016. ISBN 978-3-319-39835-8.

VARMA, V. S. et al. Stochastic maximum allowable transmission intervals for the stability of linear wireless networked control systems. In: IEEE ANNUAL CONFERENCE ON DECISION AND CONTROL (CDC), 56th., 2017, Melbourne, Australia. Proceedings... New York City: IEEE, 2017. p. 6634-6639.

WALSH, G. C.; YE, H.; BUSHNELL, G. Stability analysis of networked control systems. IEEE Transactions on Control Systems Technology, v. 10, n. 3, p. 438-446, 2002.

WONHAM, W. H. Random differential equations in control theory. In: BHARUCHAREID, A. T. (Ed.). Probabilistic Methods in Applied Mathematics. Cambridge, MA: Academic Press, 1970. v. 2, p. 131-212.

WU, Z. et al. Passivity-based asynchronous control for Markov jump systems. IEEE Transactions on Automatic Control, v. 62, n. 4, p. 2020-2025, 2017.

WU, Z.-G. et al. Asynchronous $H_{2} / H_{\infty}$ filtering for discrete-time stochastic Markov jump systems with randomly occurred sensor nonlinearities. Automatica, v. 50, n. 1, p. 180-186, 2014.

ZHANG, J.; XIA, Y.; BOUKAS, E. K. New approach to $H_{\infty}$ control for Markovian jump singular systems. IET Control Theory Applications, v. 4, n. 11, p. 2273-2284, 2010.

ZHANG, L.; BOUKAS, E. K. $H \infty$ control for discrete-time Markovian jump linear systems with partly unknown transition probabilities. International Journal of Robust and Nonlinear Control, John Wiley \& Sons, Ltd., v. 19, n. 8, p. 868-883, 2009.

ZHANG, Y.; JIANG, J. Bibliographical review on reconfigurable fault-tolerant control systems. Annual Reviews in Control, v. 32, n. 2, p. 229-252, 2008. 


\section{APPENDIX A - AUXILIARY RESULTS}

Lemma A.1 (The Schur complement, Boyd et al. (1994), Costa e Marques (1998)). Given $A_{11}$ and $A_{22}$ symmetric matrices, we have that if $A_{22}>0$, then $A_{11}-A_{12} A_{22}^{-1} A_{12}^{\prime}>$ $(\geq) 0$ if and only if $\left[\begin{array}{ll}A_{11} & A_{12} \\ A_{12}^{\prime} & A_{22}\end{array}\right]>(\geq) 0$.

Lemma A.2 (Oliveira, Bernussou e Geromel (1999)). For $P=P^{\prime}>0$, the inequality $G P^{-1} G^{\prime} \geq \operatorname{Her}(G)-P$ holds for any square matrix $G$ of compatible dimensions.

Proof. It readily follows by noting that $(G-P) P^{-1}(G-P)^{\prime} \geq 0$ holds true for all square matrices $G$.

Lemma A.3 (Elimination Lemma, Boyd et al. (1994)). Given $G$, $U$, and $V$, there exists $X$ such that

$$
G+U X V^{\prime}+V X^{\prime} U^{\prime}>0
$$

if and only if

$$
\tilde{U}^{\prime} G \tilde{U}>0, \quad \tilde{V}^{\prime} G \tilde{V}>0
$$

where $\tilde{U}$ and $\tilde{V}$ are orthogonal complements of $U$ and $V$.

Lemma A.4 (Geromel, Gonçalves e Fioravanti (2009)). For non singular $\hat{X} \in \mathbb{H}^{n+}$, $\hat{U} \in \mathbb{H}^{n}$, and $p_{i j} \geq 0, \sum_{j \in \mathbb{N}} p_{i j}=1$, we have that $\mathcal{E}_{i}\left(\hat{U} \hat{X}^{-1} \hat{U}^{\prime}\right) \geq \mathcal{E}_{i}(\hat{U}) \mathcal{E}_{i}(\hat{X})^{-1} \mathcal{E}_{i}\left(\hat{U}^{\prime}\right)$.

Proof. Note that $\hat{U}_{j} \hat{X}_{j}^{-1} \hat{U}_{j}^{\prime} \geq \hat{U}_{j} \hat{X}_{j}^{-1} \hat{U}_{j}^{\prime}$ for all $j \in \mathbb{N}$, and thus by taking the Schur complement, we get that

$$
\left[\begin{array}{cc}
\hat{U}_{j} \hat{X}_{j}^{-1} \hat{U}_{j}^{\prime} & \hat{U}_{j} \\
\hat{U}_{j}^{\prime} & \hat{X}_{j}
\end{array}\right] \geq 0
$$


Multiplying the last inequality by $p_{i j}$, summing everything up with respect to $j \in \mathbb{N}$, and applying again the Schur complement leads to the desired result.

We partition the symmetric matrix $P_{i} \in \mathbb{B}\left(\mathbb{R}^{2 n}\right), P_{i}>0_{2 n}$, as follows

$$
P_{i}=\left[\begin{array}{cc}
X_{i} & \hat{U}_{i} \\
\hat{U}_{i}^{\prime} & \hat{X}_{i}
\end{array}\right], \quad P_{i}^{-1}=\left[\begin{array}{cc}
Y_{i}^{-1} & \hat{V}_{i} \\
\hat{V}_{i}^{\prime} & \hat{Y}_{i}
\end{array}\right]
$$

where $X_{i} \in \mathbb{B}\left(\mathbb{R}^{n}\right)$ and $Y_{i} \in \mathbb{B}\left(\mathbb{R}^{n}\right)$, for all $i \in \mathbb{N}$. Furthermore, we partition $\mathcal{E}_{i}(P)^{-1}$ as follows

$$
\mathcal{E}_{i}(P)^{-1}=\left[\begin{array}{cc}
\hat{P}_{1 i} & \hat{P}_{2 i} \\
\hat{P}_{2 i}^{\prime} & \hat{P}_{3 i}
\end{array}\right]
$$

The matrix set $P$ is related to the $\mathcal{H}_{2}$ control and filtering whose starting point is (3.4). Similarly, for the $\mathcal{H}_{\infty}$ control and filtering, we partition $P_{i} \in \mathbb{B}\left(\mathbb{R}^{2 n}\right), P_{i}>0_{2 n}$, in the following form

$$
Q_{i}=\left[\begin{array}{cc}
T_{i} & \bar{U}_{i} \\
\bar{U}_{i}^{\prime} & \bar{T}_{i}
\end{array}\right], \quad Q_{i}^{-1}=\left[\begin{array}{cc}
Z_{i}^{-1} & \bar{V}_{i} \\
\bar{V}_{i}^{\prime} & \bar{Z}_{i}
\end{array}\right]
$$

Besides,

$$
\mathcal{E}_{i}(Q)^{-1}=\left[\begin{array}{ll}
\hat{Q}_{1 i} & \hat{Q}_{2 i} \\
\hat{Q}_{2 i}^{\prime} & \hat{Q}_{3 i}
\end{array}\right]
$$

We now briefly discuss the types of congruence transformations related to the filtering and dynamic output feedback problem in the following.

\section{A.1 Filtering transformations}

Note that (A.1) and (A.2) are the Lyapunov matrices related to (3.19)-(3.20) and (3.25), respectively. Due to the positive definiteness of $P$ and $Q$ we have that $Y_{i}^{-1}$ and $Z_{i}^{-1}$ can be inverted, thus we define the following matrices as in (FIORAVANTI; GONÇALVES; GEROMEL, 2008; GONÇALVES; FIORAVANTI; GEROMEL, 2009),

$$
\mathcal{T}_{i}^{(2)}=\left[\begin{array}{cc}
I & I \\
\hat{V}_{i}^{\prime} Y_{i} & 0
\end{array}\right], \quad \mathcal{T}_{i}^{(\infty)}=\left[\begin{array}{cc}
I & I \\
\bar{V}_{i}^{\prime} T_{i} & 0
\end{array}\right]
$$


such that

$$
\mathcal{T}^{(2)^{\prime}} P_{i} \mathcal{T}^{(2)}=\left[\begin{array}{cc}
Y_{i} & \bullet \\
Y_{i} & X_{i}
\end{array}\right], \quad \mathcal{T}^{(\infty)^{\prime}} Q_{i} \mathcal{T}^{(\infty)}=\left[\begin{array}{cc}
Z_{i} & \bullet \\
Z_{i} & T_{i}
\end{array}\right]
$$

We also define

$$
\mathcal{H}_{i}^{(2)}=\left[\begin{array}{cc}
\hat{P}_{1 i}^{-1} & \mathcal{E}_{i}(X) \\
0 & \mathcal{E}_{i}(\hat{U})^{\prime}
\end{array}\right], \quad \mathcal{H}_{i}^{(\infty)}=\left[\begin{array}{cc}
\hat{Q}_{1 i}^{-1} & \mathcal{E}_{i}(T) \\
0 & \mathcal{E}_{i}(\bar{U})^{\prime}
\end{array}\right]
$$

such that

$$
\mathcal{H}_{i}^{(2)^{\prime}} \mathcal{E}_{i}(P)^{-1} \mathcal{H}_{i}^{(2)}=\left[\begin{array}{cc}
\hat{P}_{1 i}^{-1} & \bullet \\
\hat{P}_{1 i}^{-1} & \mathcal{E}_{i}(X)
\end{array}\right], \quad \mathcal{H}_{i}^{(\infty)^{\prime}} \mathcal{E}_{i}(Q)^{-1} \mathcal{H}_{i}^{(\infty)}=\left[\begin{array}{cc}
\hat{Q}_{1 i}^{-1} & \bullet \\
\hat{Q}_{1 i}^{-1} & \mathcal{E}_{i}(T)
\end{array}\right]
$$

We have two cases:

a) Restricting the structure of $P$ by imposing $\hat{U}_{i}=-\hat{X}_{i}$, we get that $\hat{V}_{i}=Y_{i}^{-1}, \hat{U}_{i}=$ $Y_{i}-X_{i}$, and consequently $\hat{P}_{1 i}^{-1}=\mathcal{E}_{i}(Y)$ in (A.6). A similar reasoning can be applied to $Q$.

b) For the most general case, we note that $\mathcal{H}_{i}^{(2)^{\prime}} \mathcal{E}_{i}(P)^{-1} \mathcal{H}_{i}^{(2)}>0$. Then, by Lemma A.4, we get that $\hat{P}_{1 i}^{-1}=\mathcal{E}_{i}(X)-\mathcal{E}_{i}(\hat{U}) \mathcal{E}_{i}(\hat{X})^{-1} \mathcal{E}_{i}\left(\hat{U}^{\prime}\right) \geq \mathcal{E}_{i}\left(X-\hat{U} \hat{X}^{-1} \hat{U}^{\prime}\right)=\mathcal{E}_{i}(Y)$, and thus $\mathcal{E}_{i}(Y) \geq \mathcal{E}_{i}(Y) \hat{P}_{1 i} \mathcal{E}_{i}(Y)$. The similar reasoning can be applied to $\mathcal{H}_{i}^{(\infty)^{\prime}} \mathcal{E}_{i}(Q)^{-1} \mathcal{H}_{i}^{(\infty)}$.

\section{A.2 Dynamic output feedback transformations}

As in (GEROMEL; GONÇALVES; FIORAVANTI, 2009), we define the transformation $\mathcal{T}_{i}^{(2)}$

$$
\mathcal{T}_{i}^{(2)}=\left[\begin{array}{cc}
Y_{i}^{-1} & I \\
\hat{V}_{i}^{\prime} & 0
\end{array}\right] \text { such that } \mathcal{T}^{(2)^{\prime}} P_{i} \mathcal{T}^{(2)}=\left[\begin{array}{cc}
Y_{i}^{-1} & \bullet \\
I & X_{i}
\end{array}\right]
$$

along with the transformation $\mathcal{H}_{i}^{(2)}$

$$
\mathcal{H}_{i}^{(2)}=\left[\begin{array}{ll}
I & \mathcal{E}_{i}(X) \\
0 & \mathcal{E}_{i}(\hat{U})^{\prime}
\end{array}\right] \text { such that } \mathcal{H}_{i}^{(2)^{\prime}} \mathcal{E}_{i}(P)^{-1} \mathcal{H}_{i}^{(2)}=\left[\begin{array}{cc}
\hat{P}_{1 i} & \bullet \\
I & \mathcal{E}_{i}(X)
\end{array}\right] .
$$

We can analyse two cases:

a) Note that with the particular structure choice $\hat{U}_{i}=-\hat{X}_{i}$, we get that $\hat{V}_{i}=Y_{i}^{-1}$, 
$\hat{U}_{i}=Y_{i}-X_{i}$, and consequently $\mathcal{E}_{i}(Y)^{-1}=\mathcal{E}_{i}(X+\hat{U})^{-1}$ and $\hat{P}_{1 i}^{-1}=\mathcal{E}_{i}(Y)$ in (A.8).

b) Considering the most general case, we note that $\mathcal{H}_{i}^{(2)^{\prime}} \mathcal{E}_{i}(P)^{-1} \mathcal{H}_{i}^{(2)}>0$. Then, by Lemma A.4, we get that $\hat{P}_{1 i}^{-1}=\mathcal{E}_{i}(X)-\mathcal{E}_{i}(\hat{U}) \mathcal{E}_{i}(\hat{X})^{-1} \mathcal{E}_{i}\left(\hat{U}^{\prime}\right) \geq \mathcal{E}_{i}\left(X-\hat{U} \hat{X}^{-1} \hat{U}^{\prime}\right)=$ $\mathcal{E}_{i}(Y)$, and thus $\mathcal{E}_{i}(Y)^{-1} \geq \hat{P}_{1 i}$.

By defining

$$
\mathcal{T}_{i}^{(\infty)}=\left[\begin{array}{cc}
Z_{i}^{-1} & I \\
\bar{V}_{i}^{\prime} & 0
\end{array}\right] \text { such that } \mathcal{T}^{(\infty)^{\prime}} Q_{i} \mathcal{T}^{(\infty)}=\left[\begin{array}{cc}
Z_{i}^{-1} & \bullet \\
I & T_{i}
\end{array}\right] .
$$

along with the transformation $\mathcal{H}_{i}^{(\infty)}$

$$
\mathcal{H}_{i}^{(\infty)}=\left[\begin{array}{ll}
I & \mathcal{E}_{i}(T) \\
0 & \mathcal{E}_{i}(\bar{U})^{\prime}
\end{array}\right] \text { such that } \mathcal{H}_{i}^{(\infty)^{\prime}} \mathcal{E}_{i}(Q)^{-1} \mathcal{H}_{i}^{(\infty)}=\left[\begin{array}{cc}
\hat{Q}_{1 i} & \bullet \\
I & \mathcal{E}_{i}(T)
\end{array}\right],
$$

the previous discussion also applies. 


\section{APPENDIX B - STABILIZABILITY, OBSERVABILITY, AND COUPLED ALGEBRAIC RICCATI EQUATIONS}

Consider $A \triangleq\left(A_{1}, \ldots, A_{N}\right) \in \mathbb{H}^{n}, B \triangleq\left(B_{1}, \ldots, B_{N}\right) \in \mathbb{H}^{m, n}, L \triangleq\left(L_{1}, \ldots, L_{N}\right) \in$

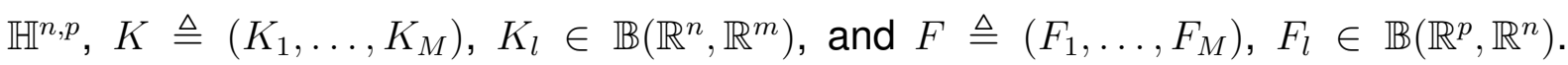
Inspired in (COSTA, 1995) and (COSTA; TUESTA, 2004), we define the concept of stochastic stabilizability and detectability as follows.

Definition B.1 (Stochastic Stabilizability). The pair $(A, B)$ is (stochastically) stabilizable if there exists $K$ such that (3.5) (or equivalently (3.6)) holds for $n_{x}=n$ and $A_{i l}=$ $A_{i}+B_{i} K_{l}$. In this case, we say that $K$ (stochastically) stabilizes $(A, B)$.

Definition B.2 (Stochastic Detectability). The pair $(L, A)$ is (stochastically) detectable if there exists $F$ such that (3.5) (or equivalently (3.6)) holds for $n_{x}=n$ and $A_{i l}=A_{i}+F_{l} L_{i}$. In this case, we say that $F$ (stochastically) stabilizes $(L, A)$.

Note that in the case of complete observation of the Markov chain, that is, $\hat{\theta}=\theta$, we would retrieve the concepts of stabilizability and detectability for MJLS given in (COSTA, 1995).

Consider scalars $\mu_{i}>0, i \in \mathbb{N}, \sum_{i \in \mathbb{N}} \mu_{i}=1, J \triangleq\left(J_{1}, \ldots, J_{N}\right) \in \mathbb{H}^{r, n}, H \triangleq$ $\left(H_{1}, \ldots, H_{N}\right) \in \mathbb{H}^{r, p}, C \triangleq\left(C_{1}, \ldots, C_{N}\right) \in \mathbb{H}^{n, q}$, and $D \triangleq\left(D_{1}, \ldots, D_{N}\right) \in \mathbb{H}^{m, q}$, and assume that $C_{i}^{\prime} D_{i}=0, H_{i} J_{i}^{\prime}=0, D_{i}^{\prime} D_{i}>0$, and $H_{i} H_{i}^{\prime}>0$ for all $i \in \mathbb{N}$. Given Definitions B.1 and B.2, we introduce two sets of discrete-time Coupled Algebraic Riccati Equations that were presented in (COSTA; TUESTA, 2004) for the study of the so-called $\mathcal{H}_{2}$ separation principle. 
Definition B.3 (Control CARE). Assume that $\hat{\theta}=\theta$. We say that $P \triangleq\left(P_{1}, \ldots, P_{N}\right) \in$ $\mathbb{H}^{n+}$ is the stochastic stabilizing solution of

$$
P_{i}=A_{i}^{\prime} \mathcal{E}_{i}(P) A_{i}+C_{i}^{\prime} C_{i}-A_{i}^{\prime} \mathcal{E}_{i}(P) B_{i}\left[B_{i}^{\prime} \mathcal{E}_{i}(P) B_{i}+D_{i}^{\prime} D_{i}\right]^{-1} B_{i}^{\prime} \mathcal{E}_{i}(P) A_{i},
$$

for all $i \in \mathbb{N}$ if (3.5) holds for $A_{i i}=A_{i}+B_{i} K_{i}(P)$, where $K_{i}(P)$ is given by

$$
K_{i}(P) \triangleq-\left[B_{i}^{\prime} \mathcal{E}_{i}(P) B_{i}+D_{i}^{\prime} D_{i}\right]^{-1} B_{i}^{\prime} \mathcal{E}_{i}(P) A_{i}
$$

for all $i \in \mathbb{N}$.

Definition B.4 (Filtering CARE). Assume that $\hat{\theta}=\theta$. We say that $S \triangleq\left(S_{1}, \ldots, S_{N}\right) \in$ $\mathbb{H}^{n+}$ is the stochastic stabilizing solution of

$$
S_{j}=\sum_{i \in \mathbb{N}} p_{i j}\left\{A_{i} S_{i} A_{i}^{\prime}+\mu_{i} J_{i} J_{i}^{\prime}-A_{i} S_{i} L_{i}^{\prime}\left[L_{i} S_{i} L_{i}^{\prime}+\mu_{i} H_{i} H_{i}^{\prime}\right]^{-1} L_{i} S_{i} A_{i}^{\prime}\right\},
$$

for all $i \in \mathbb{N}$ if (3.5) holds for $A_{i i}=A_{i}-F_{i}(S) L_{i}$, where $F_{i}(S)$ is given by

$$
F_{i}(S) \triangleq A_{i} S_{i} L_{i}^{\prime}\left(L_{i} S_{i} L_{i}^{\prime}+\mu_{i} H_{i} H_{i}^{\prime}\right)^{-1}
$$

for all $i \in \mathbb{N}$.

Conditions for the existence of stabilizing solutions to (B.1) and (B.3) are discussed in (COSTA, 1995) and rely in the concepts of stabilizability and detectability given in Definitions B.1 and B.2 


\section{APPENDIX C - NUMERICAL RESULTS OF CHAPTER 8}

The controllers and filters calculated in Chapter 8 are shown in this appendix, for clarity of presentation.

\section{C.1 Unmanned aircraft}

Some calculated structures of Section 8.1 are shown here. The $\mathcal{H}_{2}$ state-feedback controllers for $\rho_{1}=\rho_{2}=1$ are given by

$$
\left[\begin{array}{l}
K_{1} \\
K_{2} \\
K_{3}
\end{array}\right]=\left[\begin{array}{cccc}
-0.1864 & -0.0577 & 0.2259 & -0.4627 \\
-0.0311 & -0.5776 & -0.3167 & -0.1859 \\
\hline 0 & 0 & 0 & 0 \\
-0.0389 & -0.5857 & -0.2754 & -0.2409 \\
\hline 0 & 0 & 0 & 0 \\
0.0471 & 0.5908 & 0.2508 & 0.2775
\end{array}\right] \text {. }
$$

The $\mathcal{H}_{\infty}$ state-feedback controllers for $\rho=1$ and $\rho=0.5$ are shown below.

$$
\left[\begin{array}{l}
K_{1} \\
K_{2} \\
K_{3}
\end{array}\right]=\left[\begin{array}{cccc}
-0.2272 & 0.3742 & -5.0036 & -1.8111 \\
-0.0283 & -0.5239 & -0.7941 & -0.0782 \\
\hline 0 & 0 & 0 & 0 \\
0.0051 & -0.5765 & -0.0490 & 0.2057 \\
\hline 0 & 0 & 0 & 0 \\
-0.0117 & 0.5861 & -0.1002 & -0.2665
\end{array}\right]
$$


and

$$
\left[\begin{array}{l}
K_{1} \\
\hline K_{2} \\
K_{3}
\end{array}\right]=\left[\begin{array}{cccc}
-0.2181 & 0.5594 & -6.2177 & -1.6234 \\
-0.0313 & -0.6148 & -0.5769 & -0.0524 \\
\hline 0 & 0 & 0 & 0 \\
0.0040 & -0.1812 & -0.2756 & 0.0912 \\
\hline 0 & 0 & 0 & 0 \\
0.0040 & -0.1812 & -0.2756 & 0.0912
\end{array}\right] \text {. }
$$

The mixed $\mathcal{H}_{2} / \mathcal{H}_{\infty}$ state-feedback controller is given by

$$
\left[\begin{array}{l}
K_{1} \\
\hline K_{2} \\
K_{3}
\end{array}\right]=\left[\begin{array}{cccc}
-0.2576 & -0.0749 & 0.0990 & -0.7443 \\
-0.0304 & -0.6580 & -0.1104 & -0.1963 \\
\hline 0 & 0 & 0 & 0 \\
-0.0209 & -0.2264 & -0.5835 & -0.2231 \\
\hline 0 & 0 & 0 & 0 \\
0.0162 & -0.0816 & -0.3521 & -0.0770
\end{array}\right] \text {. }
$$

The $\mathcal{H}_{2}$ dynamic output feedback controller for $\rho=1$ is composed by $(\mathrm{C} .1)$ and the matrices shown below

$$
\left[\begin{array}{l}
A_{c 1} \\
\hline A_{c 2} \\
A_{c 3}
\end{array}\right]=\left[\begin{array}{rrrr}
0.0104 & -0.0023 & 0.4787 & -1.3819 \\
0.0223 & 0.4620 & 0.0407 & -0.0791 \\
0.0008 & -0.0184 & 0.3581 & 0.0098 \\
0.0229 & 0.0047 & 0.0160 & 0.3425 \\
\hline 0.5499 & 0.2064 & -0.2799 & 0.0087 \\
-0.0008 & 0.4255 & 0.1579 & -0.1975 \\
-0.0113 & 0.0168 & 0.2763 & 0.0251 \\
0.0355 & 0.0165 & -0.0179 & 0.3819 \\
0.5657 & 0.1562 & -0.1704 & -0.0052 \\
-0.0049 & 0.4574 & 0.0802 & -0.1673 \\
0.0035 & -0.0292 & 0.3761 & 0.0138 \\
0.0381 & 0.0084 & -0.0005 & 0.3800
\end{array}\right] \text {, }
$$


along with

$$
B_{c i}=\left[\begin{array}{rr}
-0.4861 & 0.0157 \\
0.8014 & 0.0206 \\
0.5651 & 0.0078 \\
-0.0172 & 0.6203
\end{array}\right]
$$

for all $i \in \mathbb{N}$.

The $\mathcal{H}_{\infty}$ dynamic output feedback controller for $\rho=1$ is given by (C.2), along with

$$
\left[\begin{array}{l}
A_{c 1} \\
A_{c 2} \\
A_{c 3}
\end{array}\right]=\left[\begin{array}{rrrr}
-0.1100 & 1.2585 & -14.9983 & -5.4427 \\
0.0286 & 0.4606 & 0.3039 & 0.1765 \\
0.0015 & -0.0337 & 0.3652 & 0.0120 \\
0.0198 & 0.0384 & -0.3810 & 0.1978 \\
\hline 0.5636 & 0.1487 & 0.0768 & -0.0293 \\
0.0232 & 0.4710 & 0.0753 & -0.0002 \\
0.0076 & -0.0435 & 0.0906 & -0.0167 \\
0.0395 & 0.0059 & -0.0162 & 0.1859 \\
0.5632 & 0.1493 & -0.2992 & -0.0189 \\
0.0274 & 0.4649 & 0.1986 & 0.1897 \\
0.0084 & -0.0446 & 0.6368 & 0.0116 \\
0.0399 & 0.0055 & -0.0015 & 0.6496
\end{array}\right],
$$

along with

$$
\left[B_{c 1}\left|B_{c 2}\right| B_{c 3}\right]=\left[\begin{array}{rr|rr|rr}
-0.4916 & 0.0561 & -0.7306 & 0.0119 & -0.3630 & -0.0019 \\
0.8343 & -0.0043 & 0.9503 & 0.1406 & 0.9223 & -0.0104 \\
0.6455 & -0.0117 & 1.0562 & 0.0680 & 0.5280 & 0.0471 \\
-0.0390 & 0.6586 & 0.0318 & 0.8289 & 0.0242 & 0.3681
\end{array}\right] .
$$

For the mixed $\mathcal{H}_{2} / \mathcal{H}_{\infty}$ dynamic output feedback controller for $\rho=0.7$, we get the fol- 
lowing state-feedback gains

$$
\left[\begin{array}{l}
K_{1} \\
\hline K_{2} \\
K_{3}
\end{array}\right]=\left[\begin{array}{cccc}
-0.1873 & -0.0583 & 0.1824 & -0.4870 \\
-0.0333 & -0.6331 & -0.3268 & -0.1605 \\
\hline 0 & 0 & 0 & 0 \\
-0.0030 & -0.2525 & -0.6102 & -0.1466 \\
\hline 0 & 0 & 0 & 0 \\
0.0061 & -0.1067 & -0.3535 & -0.0607
\end{array}\right] .
$$

\section{C.2 Mass-spring-damper system}

The mixed $\mathcal{H}_{2} / \mathcal{H}_{\infty}$ filter for $\rho=0.7$ of Section 8.2 is given by

$$
\left[\begin{array}{l|l}
A_{f 1} & B_{f 1} \\
\hline C_{f 1} & D_{f 1} \\
\hline A_{f 2} & B_{f 2} \\
\hline C_{f 2} & D_{f 2} \\
\hline A_{f 3} & B_{f 3} \\
\hline C_{f 3} & D_{f 3}
\end{array}\right]=\left[\begin{array}{rrrr|r}
-0.7409 & 0.4769 & 0.0750 & 0.1427 & 0.0488 \\
0.2111 & 0.5444 & 0.0745 & 0.3966 & 0.0025 \\
-1.7995 & 0.5762 & -0.7625 & 0.5941 & -0.5292 \\
-0.1251 & -1.3478 & 0.2475 & 0.5073 & 0.1277 \\
\hline 0.4632 & 0.0925 & 0.0126 & -0.0026 & -0.0376 \\
\hline-0.7577 & 0.4576 & -0.0305 & 0.1346 & 0.1154 \\
0.2116 & 0.5237 & 0.0741 & 0.3940 & 0.0004 \\
-1.6660 & 1.0621 & -0.0196 & 0.6867 & -0.8392 \\
-0.1457 & -1.4030 & 0.0165 & 0.4959 & 0.2517 \\
\hline 0.4629 & 0.1342 & 0.0369 & -0.0060 & -0.0543 \\
\hline-0.7410 & 0.4771 & 0.0798 & 0.1429 & 0 \\
0.2114 & 0.5463 & 0.0748 & 0.3975 & 0 \\
-1.8088 & 0.5517 & -0.8159 & 0.5776 & 0 \\
-0.1232 & -1.3475 & 0.2601 & 0.5089 & 0 \\
\hline 0.4519 & 0.0956 & 0.0107 & -0.0073 & 0
\end{array}\right]
$$

(C.10) 
and the $\mathcal{H}_{2}$ and $\mathcal{H}_{\infty}$ Bernoulli filters are given by

$\left[\begin{array}{c|c}A_{f 1} & B_{f 1} \\ \hline C_{f 1} & D_{f 1} \\ \hline A_{f 2} & B_{f 2} \\ \hline C_{f 2} & D_{f 2} \\ \hline A_{f 3} & B_{f 3} \\ \hline C_{f 3} & D_{f 3}\end{array}\right]=\left[\begin{array}{rrrr|r}-0.7562 & 0.5086 & 0.0037 & 0.1435 & 0.0861 \\ 0.2092 & 0.5604 & 0.0753 & 0.4012 & -0.0041 \\ -1.6559 & 0.0736 & -0.1873 & 0.5113 & -0.6522 \\ -0.1463 & -1.2887 & 0.0848 & 0.5140 & 0.1949 \\ \hline 0.0000 & 1.0000 & 0.0138 & 0.0000 & -0.0157 \\ \hline-0.7562 & 0.5086 & 0.0394 & 0.1435 & 0.0682 \\ 0.2092 & 0.5604 & 0.0736 & 0.4012 & -0.0032 \\ -1.6559 & 0.0736 & -0.4582 & 0.5113 & -0.5164 \\ -0.1463 & -1.2887 & 0.1658 & 0.5140 & 0.1543 \\ \hline-0.0000 & 1.0000 & 0.0072 & -0.0000 & -0.0124 \\ \hline-0.7562 & 0.5086 & 0.0791 & 0.1435 & 0 \\ 0.2092 & 0.5604 & 0.0718 & 0.4012 & 0 \\ -1.6559 & 0.0736 & -0.7588 & 0.5113 & 0 \\ -0.1463 & -1.2887 & 0.2556 & 0.5140 & 0 \\ \hline 0.0000 & 1.0000 & -0.0000 & 0.0000 & 0\end{array}\right]$

(C.11) 
and

$\left[\begin{array}{l|l}A_{f 1} & B_{f 1} \\ \hline C_{f 1} & D_{f 1} \\ \hline A_{f 2} & B_{f 2} \\ \hline C_{f 2} & D_{f 2} \\ \hline A_{f 3} & B_{f 3} \\ \hline C_{f 3} & D_{f 3}\end{array}\right]=\left[\begin{array}{rrrr|r}-0.7327 & 0.5016 & -0.0053 & 0.1396 & 0.1058 \\ 0.2227 & 0.5564 & 0.0743 & 0.3990 & 0.0020 \\ -2.0784 & 0.2007 & -0.3992 & 0.5823 & -0.5678 \\ -0.0959 & -1.3039 & 0.0947 & 0.5055 & 0.2037 \\ \hline 1.0000 & 0.0000 & 0.0709 & 0.0000 & -0.0817 \\ \hline-0.7327 & 0.5016 & 0.0627 & 0.1396 & 0.0488 \\ 0.2227 & 0.5564 & 0.0781 & 0.3990 & -0.0006 \\ -2.0784 & 0.2007 & -0.8149 & 0.5823 & -0.2422 \\ -0.0959 & -1.3039 & 0.2246 & 0.5055 & 0.0973 \\ \hline 1.0000 & -0.0000 & 0.0211 & -0.0000 & -0.0382 \\ \hline-0.7327 & 0.5016 & 0.0878 & 0.1396 & 0 \\ 0.2227 & 0.5564 & 0.0767 & 0.3990 & 0 \\ -2.0784 & 0.2007 & -0.9149 & 0.5823 & 0 \\ -0.0959 & -1.3039 & 0.2742 & 0.5055 & 0 \\ \hline 1.0000 & -0.0000 & -0.0000 & -0.0000 & 0\end{array}\right]$




\section{APPENDIX D - LIST OF PUBLICATIONS BY THE AUTHOR}

1. OliVEIRA, A. M. de; COSTA, O. L. V.; DAAFOUZ, J. $\mathcal{H}_{2}$ Dynamic Output Feedback Control for Hidden Markov Jump Linear Systems. In: IMA Volume for Stochastic Control, Computational Methods, and Applications. New York City: Springer International Publishing, 2019. To appear.

2. OlIVEIRA, A. M. de; COSTA, O. L. V.; DAAFOUZ, J. Design of stabilizing dynamic output feedback controllers for hidden Markov jump linear systems. IEEE Control Systems Letters, v. 2, n. 2, p. 278-283. doi: 10.1109/LCSYS.2018.2829883.

3. OLIVEIRA, A. M. de; COSTA, O. L. V. Mixed $\mathcal{H}_{2} / \mathcal{H}_{\infty}$ control of hidden Markov jump systems. International Journal of Robust and Nonlinear Control, v. 28, n. 4, p. 1261-1280, 2018. ISSN 1099-1239. doi: 10.1002/rnc.3952.

4. OLIVEIRA, A. M. de; COSTA, O. L. V. $\mathcal{H}_{\infty}$-filtering for Markov jump linear systems with partial information on the jump parameter. IFAC Journal of Systems and Control, v. 1, p. 13-23, 2017. doi: 10.1016/j.ifacsc.2017.05.002

5. OLIVEIRA, A. M. de; COSTA, O. L. V. $\mathcal{H}_{2}$-Filtering for discrete-time hidden Markov jump systems. International Journal of Control, v. 90, n. 3, p. 599-615, 2017. doi: 10.1080/00207179.2016.1186844.

6. OLIVEIRA, A. M. de; COSTA, O. L. V. Mixed $\mathcal{H}_{2} / \mathcal{H}_{\infty}$ filtering for Markov jump linear systems. International Journal of Systems Science, v. 49, n. 15, p. 3023-3036, 2018. doi: 10.1080/00207721.2018.1531321. 
7. OliVEIRA A. M. de; VARMA, V. S.; POSTOYAN, R.; MORARESCU, I. C.; DAAFOUZ, J.; Costa, O. L. V. Network-aware design of state-feedback controllers for linear wireless networked control systems. IFAC-PapersOnLine, v. 51, n. 16, p. 205-210, 2018. In 6th IFAC Conference on Analysis and Design of Hybrid Systems (ADHS 2018), Oxford, UK.

8. OLIVEIRA, A. M. de; COSTA, O. L. V.; DAAFOUZ, J. A suboptimal LMI formulation for the $\mathcal{H}_{2}$ static output feedback control of hidden Markov jump linear systems. EUROPEAN CONTROL CONFERENCE (ECC), 2018, Limassol, Cyprus. Proceedings... New York City: IEEE, 2018. p. 3095-3100.

9. OLIVEIRA, A. M. de; COSTA, O. L. V.; DAAFOUZ, J. Controle $\mathcal{H}_{\infty}$ por realimentação estática de saída para sistemas lineares com saltos markovianos com observação parcial. In: CONGRESSO BRASILEIRO DE AUTOMÁTICA (CBA 2018), XXII, 2018, João Pessoa, PB. Anais Eletrônicos... Campinas: SBA, 2018.

10. OLIVEIRA, A. M. de; COSTA, O. L. V. Mixed $\mathcal{H}_{2} / \mathcal{H}_{\infty}$ state feedback control for Markov jump linear systems with hidden observations. IFAC-PapersOnLine, v. 50, n. 1, p. 3800-3805, 2017. 20th IFAC World Congress, Toulouse, France.

11. OLIVEIRA, A. M. de; COSTA, O. L. V. $\mathcal{H}_{\infty}$-filtering design for discrete-time Markov Jump Systems with hidden parameters. In: IEEE CONFERENCE ON CONTROL APPLICATIONS (CCA), 2016, Buenos Aires. Proceedings... New York City: IEEE, 2016. p. 972-977.

12. OliveIRA, A. M. de; COSTA, O. L. V. Controle misto $H_{2} / H_{\infty}$ para sistemas lineares com saltos markovianos com cadeia oculta. In: CONGRESSO BRASILEIRO DE AUTOMÁTICA, XXI, 2016, Vitória, ES. Anais Eletrônicos... Campinas: SBA, 2016. p. 146-151. 